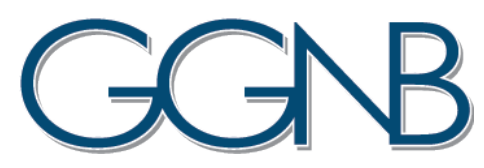

\title{
Eye, hand and space representations and causal interference in bihemispheric pulvinar-parietal circuitry
}

\author{
Dissertation for the award of the degree \\ Doctor rerum naturalium
}

submitted by

\section{Danial Arabali \\ from Gorgan, Iran}

\begin{abstract}
Faculty of Biology
Georg-August-Universität Göttingen

Doctoral program Systems Neuroscience

of the Göttingen Graduate School for Neurosciences, Biophysics, and Molecular Biosciences
\end{abstract}




\section{Thesis committee}

Dr. Igor Kagan (Supervisor)

Decision and Awareness Group, Cognitive Neuroscience Laboratory, German Primate Center, Göttingen

Prof. Dr. Melanie Wilke

Institute for Cognitive Neurology, University Medical Center, Göttingen

Prof. Dr. Hansjörg Scherberger

Neurobiology Labratory, German Primate Center, Göttingen

\section{Examination board}

Referee: Dr. Igor Kagan (Supervisor)

Decision and Awareness Group, Cognitive Neuroscience Laboratory, German Primate Center, Göttingen

Co-referee: Prof. Dr. Melanie Wilke

Institute for Cognitive Neurology, University Medical Center, Göttingen

Further members of the examination board:

Prof. Dr. Alexander Gail

SensoryMotor Group, Cognitive Neuroscience Laboratory, German Primate Center, Göttingen

Dr. Arezoo Pooresmaeili

AG Perception and Cognition, European Neurosciene Institute, Göttingen

Prof. Dr. Annekathrin Schacht

Affective Neuroscience and Psychophysiology, University of Göttingen, Göttingen

Date of oral examination: 14st of June, 2018 


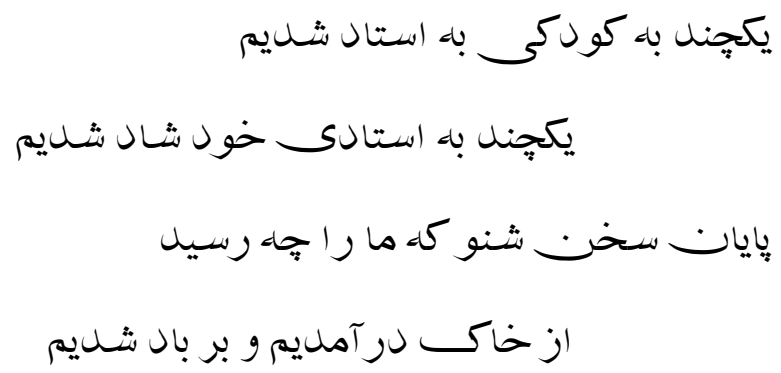

With them the seed of wisdem did I sow,

And with my own band labour'd it to grow

And this was all the harvest that I reap'd-

"I came like water, and like wind I go"

Zum Meister ging ich einst - das war die Jugendzeit -

Dann babe ich mich der eigenen Meisterschaft gefreut

Und wollt ibr wissen, was davon das Ende ist?

Den Staubgeborenen hat wie Staub der Wind zerstreut. 



\begin{abstract}
Recent research proposes that a proper action is the outcome of continuous competition between multiple frontoparietal neuronal representations of possible response options and this competition is biased by the input from other cortical or subcortical regions within and across two hemispheres. Pulvinar, the largest thalamic nucleus that has vast and complex connections with many cortical areas, is one likely candidate for having influence on action selection and specification. It is reciprocally connected with frontoparietal areas related to visually-guided eye and arm movements and its lesions cause deficits partially similar to what was observed after parietal lesions, such as spatial neglect and optic ataxia. In spite of that the information about how exactly pulvinar encodes visually-guided eye and arm motor goals and actions is very scarce. During the last few years we tried to understand the role of pulvinar in goal-directed visuomotor behavior. We compared the neuronal representations of spatial movements with eye, left or right arm in parietal cortex and dorsal part of pulvinar while monkeys performed different visuomotor tasks. In addition, we investigated the causal role of pulvinar in modulating these neuronal representations in parietal cortex by pharmacologically inactivating pulvinar in one hemisphere and simultaneously recording from parietal reach- or saccade-related areas from both hemispheres. Taken together our findings show that neuronal signals in dorsal pulvinar encode spatial as well as effector information during visual processing, motor planning and execution with considerable similarities to parietal cortex. In addition, pulvinar is causally involved in parietal action selection mechanism since its unilateral inactivation not only causes space- and limb-specific behavioral deficits but also strongly modulates the neuronal responses, as well as intraand inter-areal communication underlying visually-guided reaches and saccades in both hemispheres. Although much remains to be elucidated, both in regard to detailed properties of neuronal encoding in the dorsal pulvinar as compared to parietal cortex, and in terms of epoch- and tuning-specific effects of pulvinar inactivation, this work demonstrates that the dorsal pulvinar and parietal cortex are tightly linked functionally. Furthermore, it seems that pulvinar might be more strongly involved in mediating visually-guided reach signals than saccades, underscoring its putative role in coordinated visually-guided actions which are one of the hallmarks of behavioral repertoire in primates.
\end{abstract}





\section{Table of contents}

1 General introduction $\quad 1$

1.1 Goal-directed behavior . . . . . . . . . . . . . . . . . . 1

1.2 Effector specificity and spatial representation in frontoparietal areas . . . . . . . 2

1.3 Decision signals in frontoparietal areas . . . . . . . . . . . . . . . . 4

1.4 Thalamus: anatomy and functions $\ldots \ldots \ldots \ldots \ldots \ldots$

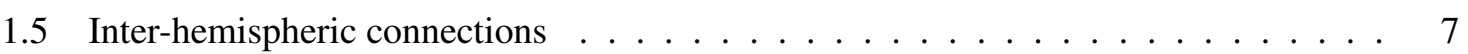

1.6 Local field potentials $\ldots \ldots \ldots \ldots \ldots \ldots$

1.7 Aims of the current thesis . . . . . . . . . . . . . . . . . . . . . 12

2 Eye, hand and space representations in cortical parietal area MIP as compared to tha$\begin{array}{lr}\text { lamic dorsal pulvinar } & \mathbf{1 3}\end{array}$

2.1 Abstract . . . . . . . . . . . . . . . . . . . . 13

2.2 Introduction . . . . . . . . . . . . . . . . . . . . . . . 14

2.2.1 Spiking activity in parietofrontal areas encode hand and space . . . . . . . . 14

2.2.2 Hand and space representations by LFP . . . . . . . . . . . . . . 15

2.2.3 Evidence from lesion studies . . . . . . . . . . . . . . . . . . . . . . . 15

2.2 .4 Reach related signals in pulvinar . . . . . . . . . . . . . . 16

2.2 .5 Aims . . . . . . . . . . . . . . . . . . . . . 17

2.3 Methods . . . . . . . . . . . . . . . . . . . . . . . . . . 17

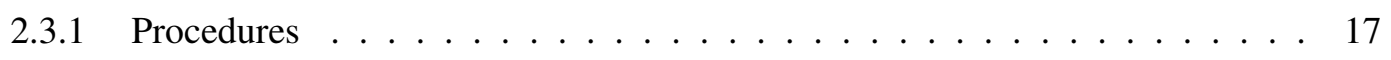

2.3.2 Animal preparation . . . . . . . . . . . . . . . . . . 17

2.3 .3 MR imaging . . . . . . . . . . . . . . . . . . . 18

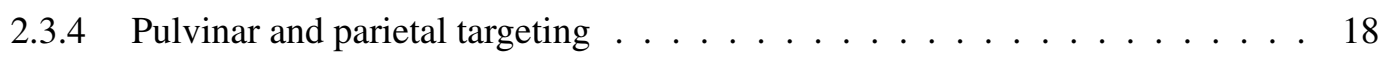

2.3.5 Electrophysiological recordings . . . . . . . . . . . . . . . . . 19

2.3.6 Behavioral paradigm . . . . . . . . . . . . . . . . . 20

2.3 .7 Data analysis . . . . . . . . . . . . . . . . . . 22

2.4 Results . . . . . . . . . . . . . . . . . . . . . . . . . 24

2.4.1 Encoding of hand-space variables in area MIP during dissociated delay reach

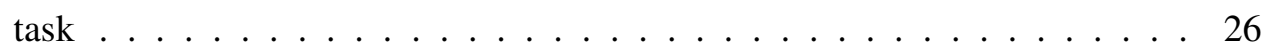

2.4.2 How are different tasks represented in MIP . . . . . . . . . . . . . 28

2.4.3 Are hand-space representations similar along IPS (posterior vs anterior locations $)$. . . . . . . . . . . . . . . . . . . 39 
2.4.4 How different are hand and space representations in MIP versus dorsal pulvinar? 41

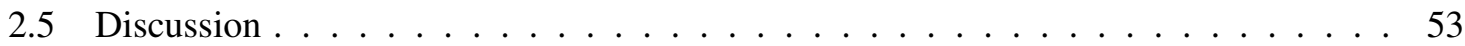

2.5.1 General differences in power spectrum across monkeys . . . . . . . . . . 53

2.5.2 Dorsal pulvinar and MIP encode eye-, hand- and space similarly . . . . . . . 54

2.5.3 Frequency specific tuning in MIP and dPul . . . . . . . . . . . . . . 55

2.5.4 Anterior and posterior MIP contribute differently to visuomotor behavior . . 56

2.5.5 Future directions and summary . . . . . . . . . . . . . . 56

2.6 Acknowledgments . . . . . . . . . . . . . . . . . . . . 57

3 Effects of dorsal pulvinar inactivation on parietal reach and saccade signals in both hemispheres

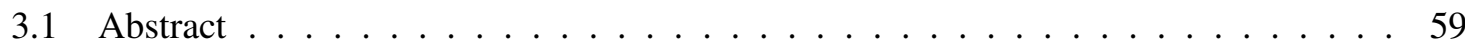

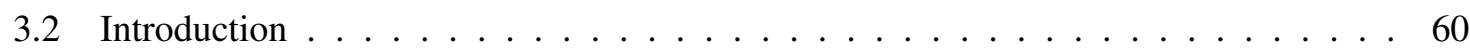

3.2.1 Task and effector encoding in dorsal pulvinar and similarities with MIP . . . 60

3.2.2 Similar deficits after MIP or pulvinar lesions _ . . . . . . . . . . . . 60

3.2.3 Thalamocortical interactions . . . . . . . . . . . . . . 61

3.2 .4 Aims . . . . . . . . . . . . . . . . . . 61

3.3 Methods . . . . . . . . . . . . . . . . . . . . . 62

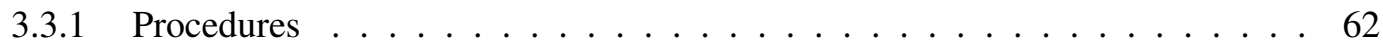

3.3 .2 Animal preparation . . . . . . . . . . . . . . . . 62

3.3 .3 MR imaging ..................... 63

3.3.4 Pulvinar and parietal targeting . . . . . . . . . . . 63

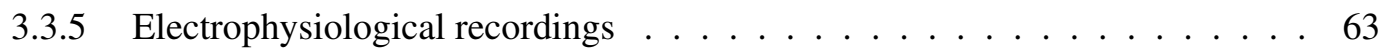

3.3.6 Reversible pharmacological inactivation . . . . . . . . . . . . . . . 63

3.3 .7 Behavioral paradigm . . . . . . . . . . . . . . . . 66

3.3 .8 Data analysis . . . . . . . . . . . . . . . . 66

3.4 Results . . . . . . . . . . . . . . . . . . . . . . . 68

3.4.1 Behavioral effects of unilateral pulvinar inactivation in the delay reach task . 68

3.4.2 Unilateral pulvinar inactivation alters single/multi-unit activities in area MIP 72

3.4.3 LFP power spectrum changed dramatically after pulvinar inactivation . . . . 78

3.4.4 Unilateral pulvinar inactivation alters single/multi-unit activities in area LIP . 93

3.4.5 Did pulvinar inactivation change LFP power in LIP similarly to MIP? . . . . 97

3.4.6 Behavioral effects of unilateral pulvinar inactivation in the delay saccade task 104

3.5 Discussion . . . . . . . . . . . . . . . . . . . . . 106

3.5.1 Unilateral pulvinar inactivation caused mostly contralesional reach and saccade deficits . . . . . . . . . . . . . . . . . . . . . . 106

3.5.2 Unilateral pulvinar inactivation alters MIP spiking activity differently in opposite hemispheres . . . . . . . . . . . . . . . . . . . 108

3.5.3 Pulvinar inactivation modulates low frequency oscillations in MIP and LIP in a task-specific manner . . . . . . . . . . . . . . . . . . . . 110

3.5.4 Pulvinar inactivation modulates LIP activity for saccades differently than MIP activity for reaches . . . . . . . . . . . . . . . . . . . . 112 
3.5 .5 Summary . . . . . . . . . . . . . . . . . . . 112

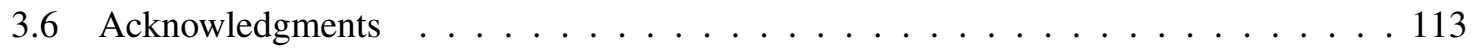

4 Changing saccade plans: response competition in spatial decision-making 115

4.1 Abstract . . . . . . . . . . . . . . . . . . 115

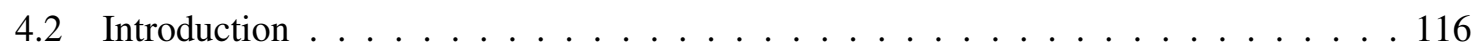

4.3 Methods . . . . . . . . . . . . . . . . . . . . . 118

$4.3 .1 \quad$ Participants . . . . . . . . . . . . . . . . . . 118

4.3.2 Apparatus and data acquisition . . . . . . . . . . . . . . 118

4.3.3 Experimental procedures and tasks . . . . . . . . . . . . . . . . . . . . . . . . . . . . . . . . . . .

4.3 .4 Data analysis . . . . . . . . . . . . . . . . . 121

4.4 Results . . . . . . . . . . . . . . . . . . . . . . . . . . . . 127

4.4.1 Slower reaction time in primary choice compared to primary instructed trials 127

4.4.2 Follow second cue task (FSC task) . . . . . . . . . . . . . . . 128

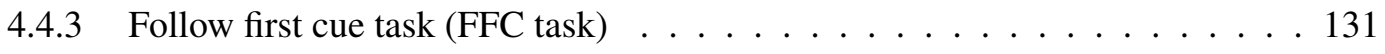

4.5 Discussion . . . . . . . . . . . . . . . . . . . . . 135

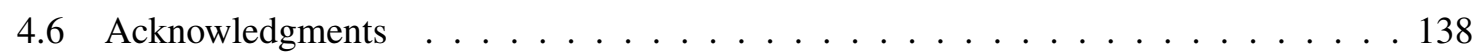

5 General discussion $\quad 141$

$\begin{array}{ll}\text { References } & 147\end{array}$ 



\section{Chapter 1}

\section{General introduction}

\subsection{Goal-directed behavior}

Our brain receives visual information through our eyes and processes it further in thalamus and primary visual cortex. Two separate pathways, dorsal and ventral streams (Goodale \& Milner, 1992) are thought to process different aspects of the sensory input in an interactive way and eventually the processed signal might cause a unique motoric response. "Ventral visual stream" or "what pathway" is an occipito-temporal network for object recognition and perception (Kravitz et al., 2013) which comprises several primary visual cortices and also projections to the temporal cortical areas. Dorsal visual stream" or "where pathway" is an occipito-parieto-frontal network which is engaged in attention, sensorimotor integration, goal directed behavior, decision-making and spatial awareness, (Andersen \& Cui, 2009; Bisley \& Goldberg, 2010; Kubanek et al., 2013; Pesaran et al., 2008; Wilke et al., 2012). This pathway includes later stages of extrastriate visual processing such as areas MT, MST and V6 which are mainly involved in the motion perception. It covers also visuomotor areas such as V6A and lateral, medial and anterior intraparietal areas (respectively LIP, MIP, AIP).

The question is how the brain deals with the problem of what and how a specific action should be done at a certain occasion. According to traditional cognitive theories, sensory information from the environment is first perceived and used to build representations of external objects (ventral stream builds what objects are available and dorsal streams builds the spatial information of objects), then cognitive processes make decision about a required action based on the current demands, incentives and previous memories, and eventually a motor plan is formed and executed. In this scheme, perception, decision making and motor commands are processed separately, and separate circuits in the brain encode action selection and specification.

But results from many recent neurophysiological studies do not fit to this proposal. Instead, (Cisek, $2007,2012)$ proposed that process of action selection and specification might occur simultaneously and behavior could be the outcome of a constant competition between neuronal representations of multiple possible action plans which he called "Affordance competition hypothesis". Cisek proposed that different potential motor goals are represented by multiple neuronal populations within frontoparietal network (for example within posterior parietal cortex (PPC)), which might compete with each other through mutual inhibition, and the information from other cortical or subcortical regions 
(including frontal cortices and also thalamus), within and across two hemispheres, influence this competition toward final action selection.

In support of this, dorsal stream seems not to build a general spatial representation but different regions within dorsal stream seem to encode action-specific spatial information and on top of that encode cognitive aspects. For example, cells in PPC have been shown to encode not only sensorimotor movement preparation information (Andersen, 1995; Colby \& Goldberg, 1999) but also attention (Bisley \& Goldberg, 2010; Colby \& Goldberg, 1999; Szczepanski et al., 2010) and decision variables such as reward expectation (Kubanek \& Snyder, 2015a; Platt \& Glimcher, 1999).

\subsection{Effector specificity and spatial representation in frontoparietal ar- eas}

As proposed by affordance competition hypothesis (Cisek, 2007), the process of action specification and selection is a continuous interaction between multiple cortical and subcortical structures until an action is released. Accordingly, brain areas in dorsal stream with reciprocal connections (for example action-specific connection between parietal and frontal areas) thought to specify multiple potential actions at the same time and while those action representations compete with each other, various influential brain signals from other regions bias one action in favor of another. Within this framework, the network of frontoparietal areas thought to represent various possible action plans. For example separate adjacent areas within parietal cortex seem to encode various visually-guided actions which suggest their role in movement planning and action specifications.

Area LIP is thought to encode visual attention (Bisley \& Goldberg, 2010), decision making (Gold \& Shadlen, 2007) and spatial targets for saccadic motor goals (Kagan et al., 2010; Snyder et al., 1997) as well as eye-hand coordination (Dean et al., 2012). Neurons in this area are reciprocally connected to frontal area FEF (frontal eye field) (Caminiti et al., 2015; Marconi, 2001). In addition, within rostral portion of parieto-occipital sulcus (POS), area V6A is connected to dorsal parietal areas and premotor areas (Gamberini et al., 2009) and is involved in arm movement planning, eye-hand coordination and even grasping (Caminiti et al., 2015).

Adjacent areas in dorsal parietal cluster (MIP, PE and PEa) within superior parietal lobe (SPL) are reciprocally connected to various motor and premotor areas. MIP is reciprocally connected to dorsal premotor area (PMd) and also to many surrounding proprioceptive and visual areas: Area $5 \mathrm{~d}$ (PE+PEc) (Seltzer \& Pandya, 1986), V6 (Shipp et al., 1998), V6A (reciprocal) (Caminiti et al., 1999; Shipp et al., 1998), VIP (Lewis \& Van Essen, 2000). In addition and interestingly, different subregions of M1 forearm cluster receive inputs from different frontoparietal areas (Dea et al., 2016).

Neurons in dorsal parietal areas are strongly involved in somatosensory integration (e.g. hand position and geometry) for movement planning (Bakola et al., 2010) and especially visually-guided arm movement planning and execution (Andersen \& Cui, 2009; Battaglia-Mayer et al., 2016). Similar functions have been reported in frontal area dorsal premotor (PMd) (Gail et al., 2009; Klaes et al., 2011).

In one of early important studies showing encoding of movement intention in parietal cortex, (Snyder et al., 1997) recorded cells from LIP and "parietal reach region" (PRR which is a functionally defined area including part of MIP and V6A) while monkeys performed memory saccade, memory 
reach and reach-saccade dissociation tasks. They found that cells in area LIP were more active for saccade movement intentions and cells in PRR were more active when planning for a reach. This finding was replicated with other studies within the same group and others (Chang et al., 2008; Cui \& Andersen, 2007; Dean et al., 2012; Gail \& Andersen, 2006; Scherberger \& Andersen, 2007).

These studies support the idea that PPC plays a functional role in planning motor goals in a body-part specific way. While consistent across many studies most of the cells recorded in PRR and MIP are more responsive for the reaches than saccade motor goals, it has been also shown in some studies that surprisingly in area LIP similar proportion of cells have preference for either reach or saccade task (Cui \& Andersen, 2007; de Lafuente et al., 2015). One possibility is that LIP with stronger connections to visual areas encodes more abstract or less effector-specific visually-guided goals and plays a role in eye-hand reference frame transformational computations than reach areas in the medial bank of intraparietal sulcus (IPS) with closer activity to motor outputs (Christopoulos et al., 2015b, 2018).

Furthermore, PRR neurons do not show only a reach target because they respond differently to a same spatial target when the movement is planned for reach with ipsilateral, contralateral or both arms (Chang et al., 2008; Cui \& Andersen, 2007; Mooshagian et al., 2017; Snyder et al., 1997). Three studies have shown consistently that neurons in PRR are not completely lateralized in hand-preference (left vs right hand). While almost half of the cells were categorized as bilateral cells (active for reaching with either arm), one third preferred reaching with contralateral arm and only the rest preferred ipsilateral arm movements(Chang et al., 2008; Chang \& Snyder, 2012; Mooshagian et al., 2017). This lateralization is less than what has been observed in PMd and motor cortex (Cisek et al., 2003; Filimon, 2010; Kermadi et al., 2000; Medendorp, 2004). In addition, (Mooshagian et al., 2017) investigated the neuronal responses of PRR cells in bimanual reaches and found that response to bimanual reaches is not a linear summation of responses for ipsi- and contralateral arm movements. Instead PRR encode a complex combination of both arm signals. Interestingly, (Kermadi et al., 2000) demonstrated that proportion of cells tuned for bimanual movement is higher in PPC than in PMd.

Although neurons in MIP and PRR encode movement planning for either arm, reach reaction times are correlated more with the neuronal responses of contralateral arm movement than ipsilateral (Chang et al., 2008). In addition, several inactivation studies reported that lesions in MIP or PRR mostly impairs movement parameters with contralesional arm or cause contralesional bias in decisionmaking, while not affecting saccade metrics such as choice bias, reaction time, movement duration and amplitude (Battaglia-Mayer \& Caminiti, 2002; Christopoulos et al., 2015b; Hwang et al., 2012, 2014; Kubanek et al., 2015; Padberg et al., 2010; Yttri et al., 2014). Most specifically, inactivation of those areas cause hand-specific ipsilesional choice bias (Christopoulos et al., 2015b; Kubanek et al., 2015), slower reaction times mostly with contralesional hand (Battaglia-Mayer et al., 2013; Padberg et al., 2010; Yttri et al., 2014), reach end point inaccuracy or shape of trajectory mostly with contralesional arm (Battaglia-Mayer et al., 2013; Battaglia-Mayer \& Caminiti, 2002; Hwang et al., 2012; Kubanek et al., 2015; Yttri et al., 2014). The apparent discrepancy between contralesional effects of inactivation and the non-lateralized tuning in SPL is still unresolved and needs more investigation.

Apart from effector-specificity, it has been shown that cells within PPC are tuned for the direction of the movement (Battaglia-Mayer et al., 2016; Fattori et al., 2005; Kagan et al., 2010; Snyder et al., 1997) and also distance to the target (Hadjidimitrakis et al., 2014). Neurons in PPC have a preference 
to encode limited spatial directions across different tasks and temporal stages within tasks for motor goals. This spatial preference is called global tuning field (GTF) (Battaglia-Mayer et al., 2016; Battaglia-Mayer \& Caminiti, 2002). Although cells in the IPL (such as area 7a and LIP) seems to be more lateralized to prefer contralateral spatial goals, cells in the MIP and PRR are less contralateral and their spatial preference is more uniformly distributed (Battaglia-Mayer et al., 2015, 2005; Kagan et al., 2010). In addition, in a recent study, (Chang et al., 2016) demonstrated that information about the spatial location of a motor goal and also information about the effector modality adds differently in LIP and PRR areas (linear summation happens in LIP but supralinear in PRR). Contrary to the previous literature, this study suggested that PRR and the frontal eye fields (area FEF) are involved in selecting a motor plan for a reach or a saccade to a particular spatial location, while LIP is not involved in effector-specific decisions and reflects more general spatial decision processing.

\subsection{Decision signals in frontoparietal areas}

Signature of encoding multiple possible actions and decision signals has been found in frontoparietal network (Cisek, 2005; Cui \& Andersen, 2007; Klaes et al., 2011; Kubanek et al., 2013; Shadlen \& Newsome, 2001). Firing of the neurons in LIP predicts eventual choice outcome and varies depending on the perceptual difficulty of the decision variable (Shadlen \& Newsome, 2001). During a delay period before a motor response in which decision-making evolves, their firing increases or decreases with time (evidence accumulation). Several bounded accumulation models such as drift diffusion model (Ditterich, 2006; Ratcliff \& McKoon, 2008) have been proposed to explain this behavior in which the bound (decision threshold) is a critical parameter for controlling the speed and accuracy of the decision. Recently (de Lafuente et al., 2015) demonstrated that the accumulation of evidence in neuronal responses also exists in the reach-area MIP but stronger when the decision is performed by an arm movement than a saccade.

Neurons in PRR and PMd have been shown to encode also reward as a decision variable (Klaes et al., 2011; Kubanek et al., 2013; Ramakrishnan et al., 2017; Ramkumar et al., 2016). For example it has been shown that the firing rate of neurons in MIP modulates by the size or type of reward associated with a reach movement and also to the schedule in which the reward was assigned to the target (Musallam, 2004; Rajalingham et al., 2014).

Recently (Kubanek et al., 2013) have shown that when monkeys had a spatial choice to select one of the bilaterally presented targets, responses in PRR and LIP were reduced as compared to single target motor goals. Especially in PRR, cells were not firing transiently to stimulus presentation and the response was more delayed in choice trials. Authors argued that visual information from early visual cortex reaches to LIP earlier and without much processing but PRR receives more processed information maybe even through LIP. Moreover, spiking activity in LIP and PRR has been also shown to predict monkeys nonspatial (e.g. effector) choices (Cui \& Andersen, 2007, 2011) which further implies that PPC plays an important role in cognitive aspects of motor planning beyond a passive sensorimotor transformation.

Encoding of multiple movement plans and the motivational factors such as reward in the frontoparietal cortex suggest that different possible movement plans encoded by subpopulation of neurons compete with each other for example through mutual inhibitions (Klaes et al., 2011; Pastor-Bernier 
\& Cisek, 2011). This competition can be biased by modulatory signals from other brain areas up to a point that eventually only one action is executed. For example reward-related signal from basal ganglia might modulate cortical areas (Schultz, 2014) or thalamic pulvinar that has been shown to causally modulate attentional signal (Zhou et al., 2016) might also have other modulatory influences on frontoparietal network of underlying action selection. In the next paragraph we will talk about anatomy and functions of higher order thalamus.

\subsection{Thalamus: anatomy and functions}

The traditional view of thalamus which suggested its role as just relaying peripheral signal to cortical areas gave its place recently to another view. Thalamus with its complex and vast connections to almost all cortical areas is proposed recently to modulate inputs from periphery and cortex in a bidirectional manner. Anatomically thalamus is subdivided into dorsal and ventral parts. Dorsal thalamus includes relay cells projecting to cortical areas and ventral thalamus includes thalamic reticular nucleus (TRN) and lateral geniculate nucleus (LGN). Nearly $75 \%$ of cells in dorsal thalamus are relay and the rest are interneurons. Relay cells are glutamatergic while interneurons and TRN cells GABAergic (Saalmann \& Kastner, 2011).

There are two types of thalamic relay cells: first order relays which receive mostly the driver input from periphery such as from retina and are projecting to lower order sensory processing areas; and higher order relays which receive driver feedforward input from layer 5 of cortex and are part of the cortico-thalamo-cortical pathway. To be more detailed, cells in layer 5 of one cortical area project to relay cells in thalamus and they project back to superficial layers of another cortical area. This feedforward inputs from the cells in cortical layer 5 is the main cortical driver input to some of the thalamus nuclei but there are also cortical layer 6 projections to thalamus (modulatory projections), while relay cells project back to cortical layer 4 cells. Cortical layer 6 projections to relay cells innervate also TRN cells. These corticothalamic projections are similar across species (rodents, cats and monkeys) (Cappe et al., 2007).

Larger volume of dorsal thalamus includes higher order nuclei such as pulvinar and medial-dorsal nucleus. Pulvinar is the largest thalamic nucleus, and it has complex and rich connections with many cortical areas, suggesting a major role in modulating the cortico-cortical communication. According to (Kaas \& Lyon, 2007; Saalmann \& Kastner, 2011), there are at least four pulvinar subregions: lateral and inferior pulvinar which are connected to ventral visual stream (with clear retinotopy), inferiomedial pulvinar which is connected to dorsal visual areas such as MT and MST, and dorsal pulvinar (dPul) which has reciprocal connections to parietal areas such as LIP and MIP and frontal areas such as PMd and FEF (Barron et al., 2015; Benarroch, 2015; Cappe et al., 2009, 2007; Hardy \& Lynch, 1992; Prevosto et al., 2010; Yeterian \& Pandya, 1985, 1989, 1997).

Pulvinar cells similar to other cells in thalamus show two modes of firing: burst and tonic. These modes may be due to the inhibitory inputs from TRN (Bokor et al., 2005) or due to state of neuromodulators such as cholinergic inputs (Varela $\&$ Sherman, 2007). These firing modes are the underlying mechanisms for gating information and modulating effectiveness of thalamocortical interactions and they depend on the level of the conscious state. For example, a relay cell transfer 
sensory information more efficient in awake than sleep or drowsiness mode in which TRN cells are active at their peak (Jones, 2009).

It has been shown that cells in $\mathrm{dPul}$ are less responsive to visual stimuli than cells in ventral pulvinar (vPul) (Robinson et al., 1986) and they are responsive to more complex visual features. Neurons in dPul have weak or no retinotopy and large receptive fields which could lay in ipsi-, contra-, or bi-lateral visual fields while vPul cells have retinotopic organization and smaller receptive fields which are mostly in the contralateral visual field (Petersen et al., 1985, 1987). Several previous studies have observed that dorsal and ventral pulvinar cells are responsive to eye-movements and they contribute to visual spatial attention (Petersen et al., 1985, 1987; Robinson et al., 1986; Saalmann et al., 2012; Zhou et al., 2016). In addition, it has been shown using different experimental techniques that pulvinar plays a role in visuomotor behavior and visually-guided reaches (Danziger et al., 2002; Dominguez-Vargas et al., 2017; Van der Stigchel et al., 2010; Ward \& Danziger, 2005; Ward et al., 2005, 2002; Wilke et al., 2013, 2018, 2010) Lesions in pulvinar cause spatial neglect (Karnath et al., 2003), a syndrome with loss of spatial awareness of the side contralateral to the damaged hemisphere, similar to lesions in parietal and temporal cortex (Caminiti et al., 2010). It has been also shown that patients with pulvinar lesions (Snow et al., 2009) or PPC lesions (Friedman-Hill et al., 2003) have difficulty to filter out irrelevant visual information.

While lesions in vPul cause severe impairments in primary visual perception as well as attentional deficits which are mainly due to the role of those neurons in relaying early visual information from periphery to the cortex (Purushothaman et al., 2012; Soares et al., 2004; Zhou et al., 2016), unilateral inactivation of dPul causes ipsilesional choice bias in saccadic target selection similar to "extinction" followed after parietal lesions (Wilke et al., 2010). In addition, increasing the saliency or motivational attribute to the impaired hemifield could set back the choice preference to normal (Wilke et al., 2013) suggesting role of dPul in higher cognitive functions such as decision making and motivation. In spite of these deficits in higher order sensorimotor processing, dysfunction of the dorsal pulvinar causes no primarily motor and visual deficits. Moreover monkeys and humans with pulvinar lesions had severe problems with contralateral arm movements and grasping similar to deficits after parietal lesions (Hwang et al., 2012; Wilke et al., 2018, 2010; Yttri et al., 2014).

As it was briefly mentioned, TRN plays a fundamental role in the thalamic networks through its inhibitory influences to certain parts of thalamus including pulvinar. TRN is a thin layer of cells in the ventral thalamus which receive input from cortical layer 6, LGN and pulvinar and only projects to LGN and pulvinar, delivering inhibitory signal. It has retinotopic organization and RF size similar to LGN. TRN neurons have high spontaneous firing rate (i.e. tonic inhibition of thalamus) and respond transiently to visual stimuli. It has been suggested to play a functional role in visual attentional modulation through enhancing or suppressing thalamocortical communications (McAlonan et al., 2008; Saalmann \& Kastner, 2011). For example, firing rate of TRN cells decreased for an attended visual stimulus compared to unattended stimulus (McAlonan et al., 2008) or lesions to TRN caused some attentional deficits suggesting that they might direct attention to spatial targets. In addition, neuronal oscillations in TRN includes theta-alpha (7-15 Hz, spindles; Steriade et al., 1986), as well as higher beta-gamma bands (Pinault \& Deschênes, 1992) which might facilitate inter-areal communications through beta-gamma phase resetting (Saalmann \& Kastner, 2011). These oscillations might serve as thalamocortical oscillatory pacemaker (Destexhe \& Sejnowski, 2003). 
The function of the cortico-thalamo-cortical communications is not fully understood and captured attention among scientists in the last decades. It is proposed that motor output projections from layer 5 of cortex which innervate thalamus might act as efferent copies of an ongoing movement-related signal which is passing to other higher order cortical areas through thalamus for further processing and accordingly altering the behavior. This implies that transthalamic pathways connect lower order with higher order cortical areas in a hierarchical manner to transfer information needed for a potential revision in action (Sherman, 2017).

One way to achieve this is through synchronized oscillatory activities which functionally link cortical areas together. An example is studies that show lesions to higher order thalamus like pulvinar cause desynchronization between linked cortical areas in cognitive tasks. (Wróbel et al., 2007) have shown that beta band synchrony between pulvinar and visual cortex of cats increases in a spatial discrimination task. Also, inactivation of cat pulvinar caused desynchronization in visual cortex (Shumikhina \& Molotchnikoff, 1999) or inactivation of pulvinar decreased attentional modulation in visual cortical areas (Zhou et al., 2016). In a recent study, (Saalmann et al., 2012) investigated the neuronal communication between pulvinar and visual areas V4 and temporo-occipital area (TEO). They showed that the LFP-LFP coherency between pulvinar and cortical areas increases in the alpha band during attentional process. Applying Granger causality analysis, they reported higher influence of pulvinar on cortex than cortico-cortical interactions in the alpha band.

To summarize, despite the progress mentioned above, the mechanistic understanding of functionality of cortico-thalamo-cortical interactions within frontoparietal network supporting goal- directed actions is largely lacking. In that respect there is no direct evidence for the potential influences of $\mathrm{dPul}$ on the neuronal activity and communications in parietal and/or frontal reach-related areas. The current thesis aims to shed light on this issue, for the parietal regions.

\subsection{Inter-hemispheric connections}

Two hemispheres in mammalian brain connect with each other mostly through corpus callosum (CC) but also through anterior commissure (AC) (Suárez et al., 2014). Approximately 2-3\% of all cortical cells project to the opposite cerebral hemisphere (Lamantia \& Rakic, 1990). There is a topographical organization within $\mathrm{CC}$ related to the cortical connections and accordingly different parts of CC transfer different information across hemispheres. $\mathrm{CC}$ is divided into three main parts, as genu (in the anterior part connecting prefrontal areas), midbody (connecting motor, somatosensory and auditory cortices) and the third part containing isthmus (connecting superior temporal and inferior parietal areas) and splenium (connecting associative parietal and occipital areas) (Aboitiz, 1992; Lamantia \& Rakic, 1990; Pandya et al., 1971; Suárez et al., 2014). It has been shown that PFC and PPC occupy the largest CC areas among other cortical areas (Zarei et al., 2006). Prefrontal and parietotemporal areas connect with smallest fibers through genu and anterior splenium which cause slow callosal conductions. On the other side, motor and somatosensory areas connect with thicker fibers through midbody of $\mathrm{CC}$ which causes faster conductions. In general, posterior locations are more responsible to transfer sensory information and anterior regions higher order cognitive information.

During evolution as brain size increased, the anatomical connectivity between hemispheres through $\mathrm{CC}$ and $\mathrm{AC}$ decreased proportionally but in opposite, the intrahemispheric connectivity increased. 
This is in line with the fact that in more complex brains, two hemispheres are more independent (Rilling \& Insel, 1999). Moreover, studies have shown that speed of callosal information transfer is limited in larger brains like human which in opposite makes intrahemispheric communications faster and more reliable (Nowicka \& Tacikowski, 2011).

Fibers from posterior parietal cortex (PPC) pass the posterior to middle part of the CC to reach the other hemisphere (Pandya \& Vignolo, 1969; Seltzer \& Pandya, 1983). It has been shown in primates that most of the callosal fibers from area 5 within PPC predominated in layer 3 but also many in layer 4 and less in layer 5 (Caminiti \& Sbriccoli, 1985). In addition, projections through CC land mainly in layer 4 of other cerebral cortex (Essen et al., 1982). These connections are either homotopic (connected to similar structures across hemispheres), or heterotopic (connected to other structures). For example thalamic relay cells can be influenced from cortical cells in both hemispheres (Li \& Ebner, 2006). Or PRR area has transcallosal connections with itself, as well as with contralateral PMd. However, the interhemispheric projections from PRR are considerably weaker than those in frontal areas (Pandya \& Vignolo, 1969; Seltzer \& Pandya, 1983). Recently, (Shen et al., 2015) showed that interhemispheric functional connectivity is stronger in homotopic than heterotopic connections and they are consistent across conditions and stable over time, which implies major role of CC in maintaining functional crosstalk between two hemispheres.

Failure in development of corpus callosum causes severe behavioral and social deficits in humans (Paul et al., 2007). Patients with undeveloped CC show diminished interhemispheric functional connectivity (Quigley et al., 2003). In addition, changes in the morphology of CC is associated with different disorders (Zarei et al., 2006) such as motor neuron disease (Yamauchi et al., 1995), Alzheimer (Hensel et al., 2002) or even handedness of subjects (Witelson \& Goldsmith, 1991).

In split-brain patients each hemisphere is impaired to control its ipsilateral hand because the intact callosal connections are needed for integration of control signals from other hemisphere (Gazzaniga et al., 1967). Each hemisphere receives ipsilateral and contralateral motor projections but contralateral projections are stronger and ipsilateral projections are only for proximal musculature (for reaching arm movements) and not distal (for grasping or hand shaping). It has been shown that subjects' movement timing was affected after callosotomy when they moved both arms simultaneously (Fling et al., 2008) but splitting two hemispheres does not affect saccades to either side of space. Callosotomy has been used to treat patients with epilepsy. This procedure helped by suppressing the malfunction of interhemispheric information exchange (e.g. overexcitement), but on the other hand those patients with disrupted callosal connections had problems to interpret properly the environment (for example trouble in spelling the name of object which they recognize correctly).

These evidences imply that communication between brain regions within and across two hemispheres is necessary to bind different attributes and associate them with a unique object or action. When the brain faces with multiple potential responses, there will be groups of neurons which prefer certain spatial response interacting with other groups with a different preference. Consequently the process of decision-making would be the integration of information across groups with similar preference and/or competition between groups with opposite preferences. These interactions might be happening not only across groups within one hemisphere but also across two hemispheres. However, direct neurophysiological evidence for such interactions is still very scarce. More experiments are required to qualify the level of these hypothetical intra- and inter-hemispheric communications. 


\subsection{Local field potentials}

A recording microelectrode in the extracellular medium captures time-varying fluctuations of electrical potentials with respect to a reference potential. This recorded continuous signal contains wide range of spectral components. While the higher frequency spectrum of extracellular activity ( $>500 \mathrm{~Hz}$ ) is referred to spiking activity, lower frequency activities are called local field potentials (LFP; $<300 \mathrm{~Hz}$ ). Although it is called "local", it is still under debate how local this signal is (Buzsáki et al., 2012; Kajikawa \& Schroeder, 2011; Katzner et al., 2009). In short, the LFP represents a sum of various extracellular activities around the electrode tip and thought to reflect inputs to a neuronal assembly in a region while spikes reflect output of the system (Buzsáki et al., 2012). It has been shown also that LFPs predict better BOLD responses than spiking activities and both fMRI and LFP signals are mostly reflecting neuronal inputs than pyramidal cell outputs (Logothetis, 2008).

All types of transmembrane currents, including fast and slow fluctuations contribute to this extracellular field. The two characteristics of LFP signal, i.e. amplitude and frequency, depend on the proportion of different contributions of these various sources. One of the major contributors is the synaptic activities. Influx/outflux of ionic neurotransmitters through cell membrane cause sink-source currents which decay proportional to the distance to the source. In addition, long-lasting calcium spikes and also intrinsic voltage-dependent membrane responses also contribute to oscillations of membrane potential. Another source is the spike afterhyperpolarization (AHP) which contribute indirectly and usually after burst of spikes to the extracellular field (Buzsáki et al., 2012). For example, synchronized AHPs are associated with the up and down cortical states during non-REM sleep in which e.g. down states produce LFP delta waves. It has been also shown that non-neuronal cells contribute to LFP oscillations. For example glia-neuron interactions influence the very slow field oscillations.

Since any transmembrane current contribute to LFP, action potentials might also indirectly influence LFPs. Higher spike rates and synchrony increases the field power in higher frequencies (Ray \& Maunsell, 2010; Watson et al., 2017). Recently, (Waldert et al., 2013) showed that the shape (e.g. width), SNR and firing rate of the spikes can cause leakage to the LFP signal even up to the low frequency ranges. According to their study, presence of spike trains causes an increase in the amplitude of broad range of frequencies, but depending on the waveform. In addition, spikes with broader waveforms contribute more than short spikes to lower frequency LFPs. In general, the fact that many factors contribute to LFPs makes its functional interpretation more difficult than spiking activity.

Locality of spatial spread of LFPs is still controversial. While (Katzner et al., 2009) showed that in macaque visual cortex LFP is predominantly local and stays similar within $250 \mu \mathrm{m}$ around the tip of recording electrode, (Kajikawa \& Schroeder, 2011) reported that in macaque auditory cortex LFP spread far beyond that range and even extend millimeters away from auditory cortex. Other studies reported various amount of propagation across cortex from hundreds of micrometers to several millimeters (Kreiman et al., 2006; Liu \& Newsome, 2006; Logothetis et al., 2001).

By applying frequency decomposition methods LFP signal is usually divided to separate frequency bands, i.e. delta $(2-4 \mathrm{~Hz})$, theta $(4-8 \mathrm{~Hz})$, alpha $(8-12 \mathrm{~Hz})$, beta $(12-30 \mathrm{~Hz})$, and gamma $(30-100 \mathrm{~Hz})$. This has been shown by many various experiments that these different bands contribute independently 
to different behavioral functions. Brain oscillatory activities are associated with different sensory, motor and cognitive functions. Delta band is associated with deep sleep and motivation (Steriade et al., 1993). For example high amplitude delta oscillations are suggested to be caused by thalamic burst firing mode during drowsiness and low-attentive states (Jones, 2009). Theta band is important for learning and memory and is abundant during spatial exploration and REM sleep in hippocampus (Buzsáki, 2002) and is present in thalamocortical interactions (Hooks et al., 2013; Igarashi et al., 2013). Alpha oscillations with peak around $10 \mathrm{~Hz}$ are one of the major oscillatory components in the human brain. Alpha- and beta band activities $(8 \mathrm{~Hz}<\mathrm{frq}<30 \mathrm{~Hz})$ are the only rhythms which respond to events with either positive or negative modulations (called event-related synchronization or desynchronization, ERS or ERD) while the other rhythms respond mostly with ERS. This ERSERD dual response in alpha-beta range is associated with the inhibitory-disinhibitory functions (Klimesch, 2012). One hypothesis is that suppressed alpha oscillations in task-relevant areas enhance the functional processing while enhanced alpha waves suppress task irrelevant information. In one study in monkeys, (Haegens et al., 2011) showed that the spike-field coherence and power in alpha band in cortical brain areas were anticorrelated with spike rates in a task-dependent manner. Alpha band seems to be also important in thalamocortical interactions for attention (Saalmann et al., 2012).

Beta band activity was classically associated with sensorimotor processes (Pfurtscheller et al., 1996). Many studies reported beta modulations in motoric tasks in motor-related areas such as motor, premotor and parietal cortex as well as basal ganglia Khanna \& Carmena (2015). Several studies have shown that beta activity is stronger during steady states such as holding periods prior and after movements and is suppressed during movement preparation and execution in which faster oscillations are stronger (Hwang \& Andersen, 2011, 2012; Zhang et al., 2008).

According to observations that beta band is highest in idling state in which no active motor preparation is happening (Stetson \& Andersen, 2014) and lowest during high spike rates such as movement, it suggested that beta band activity might act as a motor suppressing mechanism and might be related to the maintenance of "status quo" (Engel \& Fries, 2010) which reflects the facilitation of proprioceptive feedback processing in the sensorimotor system (Zhang et al., 2008) and it is stronger when this maintenance is predicted. For example beta band power in a movement preparation delay period is higher when the reaction times are slower and vice versa (Dean et al., 2012). It has been shown that beta band synchrony increases when a sensory event is expected. Also, (Buschman \& Miller, 2007) compared oscillatory responses in bottom-up versus top-down attention and found that beta band coherency is high in top-down but gamma band in bottom-up attention. In addition, (Gilbertson et al., 2005) demonstrated that beta band synchrony in motor cortex increases with lower movement performance and slower reaction times consistent with other studies which reported excessive beta synchrony in cortical and subcortical regions when motor behavior is impaired e.g. in Parkinson's disease (Crowell et al., 2012; Kühn et al., 2004; Little \& Brown, 2014; Swann et al., 2012). Besides that, beta band synchrony in the frontoparietal network plays a role in highly cognitive processes such as decision making (Hawellek et al., 2016; Pesaran et al., 2008).

Gamma band amplitude and coherence modulations are related to various brain process such as movement preparation (Lu et al., 2015), sensory integration (Bertrand et al., 2001; Womelsdorf et al., 2006), attention (Fries et al., 2001) and consciousness (Buzsáki \& Schomburg, 2015; Engel et al., 1999; Fries et al., 2007; Singer \& Gray, 1995). Gamma-band synchronization has been associated 
with rhythmic inhibition of pyramidal cells (spike-phase encoding) (Fries et al., 2007) similar to theta phase precession phenomenon (Buzsáki, 2002). In addition, several neurological diseases have been associated with abnormal gamma oscillations such as Alzheimer, Autism and Epilepsy (Uhlhaas \& Singer, 2006).

A flexible behavior is the output of systematic communications not only within but also across neuronal assemblies. Groups of neurons with oscillatory activity can transfer information efficiently to other groups only if the activity of both groups are coherent with each other. In other words, firings of cells should be received by the other cells at the time that those cells are temporally ready to fire otherwise the received signal would not be effective enough. Therefore neuronal synchrony has been suggested to have a functional significance in all brain processes such as sensory, motor and cognition (Fries, 2005, 2015; Fries et al., 2001).

One of the current hypotheses is "binding by synchrony" (Singer, 1999) which suggests two groups of cells with near-zero phase lag activity goes into oscillatory synchrony to encode different attributes of the stimuli. In that respect many studies have been showing the role of gamma band synchronization in visual perception and attention (Bertrand et al., 2001; Engel et al., 1991; Fries et al., 2007).

Another hypothesis "communication through coherence" (Fries, 2005) proposes that effective neuronal communications will be achieved when inputs arrive at the receiver at the highest excitability level. Therefore "strong effective connectivity requires rhythmic synchronization within pre- and postsynaptic groups and coherence between them". For example, gamma synchronization which is abundant in visual cortex emerges through interaction between excitatory-inhibitory groups. Excitatory cells activate local inhibitory cells within a short delay which causes longer delays for the next firing of excitatory cells. This short excitation followed by longer inhibition acts as a gate for the postsynaptic group of cells to receive information with the same delays as in presynaptic group. This causes a phasic relationship between pre- and postsynaptic group of cells. A new proposal from the same author is formulated such that this coherent neuronal communications works both unidirectionally and bi-directionally for feedback loops (Fries, 2015).

Spike-field and field-field synchronization have got great interest recently for understanding the neuronal communications underlying various sensorimotor and cognitive tasks. For example distributed locations in monkey sensorimotor cortex have coherent activity in the beta band during contralateral hand lever press (Brovelli et al., 2004) or frontoparietal spike-field coherence in the beta band reveal free-choice decision-making in monkeys (Pesaran et al., 2008). In another study, (Saalmann et al., 2012) investigated thalamocortical interactions in attention. They found that pulvinar spike-field coherence in alpha band increases when a visual cue appears inside their receptive field and using granger causality method they showed that during attention influences of pulvinar on V4 increases in that frequency band. Also recently it was demonstrated that pulvinar inactivation reduced the effects of attention on field-field coherence between visual areas (Zhou et al., 2016).

In addition to intra- and inter-area communication within a frequency band, there are also several methods to understand how activities in different frequency ranges interact with each other including phase-phase and phase-amplitude "cross frequency coupling" (Canolty \& Knight, 2010; Jensen \& Colgin, 2007; Jirsa \& Müller, 2013). For example phase of low frequencies (e.g. theta) and amplitude of high frequencies (eg. gamma) are shown to be synchronized in different brain structures but 
extensively studied in hippocampus with a functional role in memory formation and learning (Buzsáki, 2002; Lega et al., 2016)

In summary, efficient neuronal integration across different spatial (local versus long-distant groups) and temporal (relative timing of excitability) scales is essential for effective behavior.

\subsection{Aims of the current thesis}

This thesis is composed of three studies: the first two are electrophysiological studies on macaque monkeys and the last is a human psychophysics study.

In the first study, we investigate eye, hand and space representations in local field potential oscillations in the cortical area MIP as well as dorsal part of subcortical nucleus pulvinar in thalamus. These regions are reciprocally connected with each other. We recorded neuronal activities from two macaque monkeys while they performed three tasks with different requirements in terms of hand and eye movement planning and execution. We aim to understand how effector-specific (eye vs. left hand vs right hand), and task-specific (dissociated saccade, dissociated reach, free-gaze (mostly coordinated) reach) is LFP signal in MIP and pulvinar, to what extent LFP signals are spatially tuned, and whether this potential tuning is different in the pulvinar as compared to MIP.

In the second study, we combined electrophysiology with reversible pharmacological inactivation to shed light on the causal role of dorsal pulvinar in the neuronal encoding of hand, eye and space in parietal areas MIP (and LIP) in both hemispheres. In this challenging experiment we recorded neuronal activities (spikes and LFPs) in parietal cortex bihemispherically before and after unilateral inactivation of pulvinar while one macaque monkey performed either dissociated reach or saccade task. If pulvinar is actively modulating parietal neuronal responses for the arm and/or eye movements, we expected to see significant changes in those responses after inactivation. We hypothesized that these potential neuronal changes would be different across hemispheres and intact hemisphere might show some compensatory effects or overactivation due to release from inhibitory interactions.

In the third study, we conducted a human psychophysics experiment in which we studied the saccadic decision-making behavior and how a new relevant or irrelevant visual information can influence this decision-making and motor preparation. More specifically we aimed to understand three interesting aspects: free-choice target selection, changing saccade plans during instructed or free choice conditions, and inhibition of invalid or irrelevant changes during the same conditions. 


\section{Chapter 2}

\section{Eye, hand and space representations in cortical parietal area MIP as compared to thalamic dorsal pulvinar}

\subsection{Abstract}

For a goal-directed movement, sensory information about the environment should be integrated with available information about acting effector so that the output movement will be proper and efficient. The required transformations of sensory signals into motor action plans are processed by a distributed network of neurons in cortical and subcortical regions not only within but also across both hemispheres. For example, separate groups of neurons inside posterior parietal cortex (PPC) encode spatial movement plans for different effectors (e.g. eye, left/right hand) and are reciprocally connected with frontal areas which are also partially effector-specific. In addition, most cortical areas, including the frontoparietal circuitry, are also indirectly connected through thalamus. This suggests that thalamus might play a role in actively mediating sensorimotor transformations. Although many studies investigated space- and limb-specificity in parietal cortex, at least at the level of spiking activity, this knowledge in higher order thalamus including pulvinar is very scarce. Most of our understanding about role of pulvinar in goal-directed behaviors such as performing hand movements comes from lesion studies in monkeys and humans, which show behavioral deficits partially similar to impairments after parietal lesions, such as spatial neglect and optic ataxia. The neurophysiological assessments of pulvinar visuomotor encoding are few and far between. To fill this gap, in this study we aimed to shed light on the eye-, limb-, and spatial encoding by the spiking activity and local field potential signal in cortical area MIP as well as dorsal part of pulvinar (dPul). We recorded neuronal activities from two macaque monkeys (three hemispheres) while they performed three tasks with varying level of eye-hand coordination: dissociated delayed reach (Ddr), dissociated delayed saccade (Dds) and free-gaze delayed reach (Ddf) tasks. So far the focus of analysis has been on the LFPs; the spiking activity analysis is not in the scope of analyses presented in this chapter. Using time-frequency spectral analysis of LFPs, we found that dPul and MIP represent both hands and share considerable similarities in their power spectra for encoding space and effectors. In both regions beta and gamma frequency modulations are the largest but theta band is more present in pulvinar than MIP. Our results also show that in both regions beta band activity during planning a reach decreased more for the contralateral arm while theta and gamma bands are mostly tuned for contralateral space especially after cue and during the reach movement, although the level of modulation differed across monkeys. In addition, both MIP and dPul seem to be modulated more similarly but also more frequency-specific in reach than in saccade task, implying that dPul might be more reach specific than saccade specific. Taken together, our data suggest that dorsal pulvinar participates in the encoding of space and limb in visually-guided movement planning, motivating further investigations of its causal role in shaping cortical neuronal representations. 


\subsection{Introduction}

\subsubsection{Spiking activity in parietofrontal areas encode hand and space}

For a goal-directed movement, available sensory information should be integrated with available effector information so that the output movement will be proper and efficient. For example, a single arm movement toward a specific object on the table needs several stages of transformation of the visual information about the shape and location of the object projected on the retina to a proper reach motor goal which is eventually executed by one or both hands. These transformations are processed by a distributed network of neurons in cortical and subcortical regions not only within but also across both hemispheres. Separate groups of neurons inside posterior parietal cortex (PPC), including the dorso-medial part of Superior Parietal Lobule (SPL) encode different parameters of reach motor goals. Several studies in monkeys and humans investigated effector specificity and spatial preference in different sub-populations inside SPL such as regions denoted as area 5, MIP and V6A according to some parcellations/classification, or areas PE, PEa, PEc according to other atlases (Chang et al., 2008; Cui \& Andersen, 2007; Dean et al., 2012; Medendorp, 2004; Snyder et al., 1997). These areas have been shown to be actively involved in visuospatial transformation toward motor preparation and executions of arm movements and to less degree for eye movement.

Parietal reach region (PRR) is a functionally defined area including part of MIP and V6A (Snyder et al., 1997), in which cells are more active for arm movements than saccades and contain both visuomotor and motor class responses (Gail \& Andersen, 2006; Hwang \& Andersen, 2011, 2012; Kuang et al., 2015). Spatial direction encoding by spiking activity and local field potentials (LFP) in PRR has been shown before (Chang et al., 2008; Dean et al., 2012; Hwang \& Andersen, 2011, 2012; Scherberger et al., 2005; Snyder et al., 1997). But unlike in primary motor cortex and to less extent in premotor area in which space representation is more contralaterally biased, population spike and LFP responses in PRR encode both ipsi- and contralateral space (Hwang \& Andersen, 2011, 2012; Scherberger et al., 2005). In addition to spatial tuning, studies in humans and monkeys have shown that both arms are represented in parietal cortex. According to (Medendorp, 2004) and (Filimon, 2010), in both hemispheres in humans primary motor area M1 shows significantly higher contralateral hand tuning and to less degree also premotor area PMd, but in IPS there is both ipsi and contralateral hand tuning almost equally. In respect to spiking activity in monkeys, the study by (Kermadi et al., 2000) found that $61 \%$ of cells in monkey PPC had directional spatial modulation to both sides of space and $74 \%$ of cells had modulation for movement of both arms (bimanual cells).

In a recent study, (Chang et al., 2008) investigated explicitly whether reach target representations in PRR are limb-specific. They trained monkeys to perform memory-guided reach (either with ipsior contralateral arm) and saccade tasks. Sustained activity during memory period for most of cells, but not all, was higher during reaches than saccades (similar to previous studies, e.g. (Snyder et al., 1997)). Most of the cells had significant modulation during memory period when reaches with any arm (49\%, bimanual), many cells had higher modulation with contralateral arm (34\%) and the minority with ipsilateral arm (17\%). In addition, they found cells with contralateral space preference as many as ipsilateral space preference ( $40 \%$ vs $43 \%$ ) while a minority showed preferred direction around midline. Interestingly, limb-specific cells did not have spatial preference at the population level, i.e. nearly equal number of cells showed ipsi- or contralateral space preference. 
In another study from the same group, (Chang \& Snyder, 2012) replicated these results and confirmed that limb-specificity is a continuous measure covering the whole spectrum between ipsi, bi- and contralaterally tuned cells although the tendency at the population level is toward higher number of contralateral arm specific cells. Moreover, they found that while most of bilateral-arm cells encode reaches in a gaze-centered reference frame, some of unilateral-arm cells encode in handcentered and some in gaze-centered reference frames with an overall tendency toward gaze-centered frame at the population level.

In line with previous studies (Chang et al., 2008; Chang \& Snyder, 2012), (Mooshagian et al., 2017) show that PRR cells encode ipsi-, contra- and both arms, but they are more effective in visuomotor processing when contralateral arm is recruited. In addition reach reaction time is correlated more with the neuronal responses related to contralateral arm movement than ipsilateral (Chang et al., 2008).

\subsubsection{Hand and space representations by LFP}

Apart from spiking activity, space and especially hand representations in PRR and MIP LFPs have not been studied extensively. The study of Scherberger and colleagues (Scherberger et al., 2005) raised specifically the question about movement encoding by LFPs in PRR. They showed that spiking activity and LFPs with higher beta and gamma band oscillations $(>20 \mathrm{~Hz})$ within PRR are spatially tuned toward contralateral space.

Another study (Hwang \& Andersen, 2011) have shown that lower and higher frequency bands were spatially tuned to different spatial directions (ipsilateral in lower frequencies and contralateral in higher frequencies) which was consistent across individual LFP sites. This "anticorrelation", or the opposite tuning, has been shown before (Belitski et al., 2008; Fries et al., 2001; Hwang \& Andersen, 2009). Previous studies also showed that unlike spiking activity, LFP oscillations could not be classified as visuomotor and motor groups (Hwang \& Andersen, 2009; Kuang et al., 2015). In another study from the same authors (Hwang \& Andersen, 2012), LFPs with lower frequencies $(<40 \mathrm{~Hz})$ had spatial tuning opposite to the spikes but LFPs with higher frequencies $(>40 \mathrm{~Hz})$ had almost identical spatial preference to spikes. They also found low correlation between reach goal tuning strength in spikes and LFPs recorded from the same depth, which implies different content of information by two signals. Although few studies (as mentioned above) investigated the spatial encoding in PRR LFPs, no study to our knowledge systematically investigated the effector specificity (left hand vs right hand vs eye) and its combination with spatial tuning in LFPs.

\subsubsection{Evidence from lesion studies}

Interestingly, although space and hand representations do not seem to be strongly lateralized in MIP/PRR in both spikes and LFPs, lesions to those areas cause mostly contralesional effects. Lesion or inactivation of dorsomedial areas in SPL (area 5, MIP, PRR, V6A) leads mostly to arm movement related deficits but no effects on eye movement parameters. More specifically, inactivation of those areas cause hand-specific ipsilesional choice bias (Christopoulos et al., 2015b; Kubanek et al., 2015), slower reaction times and reach inaccuracy mostly with contralesional hand (Battaglia-Mayer et al., 2013; Battaglia-Mayer \& Caminiti, 2002; Hwang et al., 2012; Kubanek et al., 2015; Padberg et al., 2010; Wilke et al., 2018, 2010; Yttri et al., 2014). 
Areas within PPC (e.g. PRR) have tight anatomical connection between each other, but also with certain frontal areas including premotor cortex (e.g PMd) (Tanne-Gariepy et al., 2002) and motor cortex (e.g. it has been shown that different subregions of M1 forearm cluster receive inputs from different frontoparietal areas (Dea et al., 2016). This apparent discrepancy between the more lateralized effects of causal perturbations versus more bilateral representations of hand and space in PPC is not yet understood.

\subsubsection{Reach related signals in pulvinar}

In addition to cortico-cortical connectivity, most cortical areas, including the frontoparietal circuitry supporting goal-directed actions, are not only directly connected to each other but also indirectly through thalamus (so called "replication principle", (Shipp, 2003)). This suggests that thalamus may play a role in actively modulating reach-related signals. For example, dorsal part of pulvinar (dPul), which is the largest thalamic nucleus, seems to be not retinotopic and is connected to dorsal cortical areas which are driving goal-directed behavior such as parietal and frontal areas involved in eye- and arm-movement planning (Barron et al., 2015; Benarroch, 2015; Cappe et al., 2007; Wilke et al., 2010).

One possibility is that pulvinar acts as a modulator for better communication between interconnected parietal areas for a certain visually-guided behavior (Zhou et al., 2016). It would suggest that pulvinar may also actively participate in forming the signals about arm movement planning, preparation and execution, instead of just passively receive a copy of ongoing commands. The knowledge about space-hand representations during visually-guided goal-directed actions is however very scarce. Most of our knowledge about role of pulvinar in goal-directed hand movements come from lesion studies in monkeys and humans (Mundinano et al., 2018; Wilke et al., 2018, 2010).

In addition, there were only three studies from the same group in 1980-1990 that investigated neurophysiological evidence toward specifically this question. In the first study, Acuna and colleagues (Acuna et al., 1983) recorded neuronal activities from groups of cells located in the oral and upper parts of the lateral, the medial, and, to a lesser extent, the inferior nuclei of the pulvinar in two Cebus apella monkeys (capuchin, New World primate), while they performed a variant of delayed saccade and reach tasks. This study has shown that many cells in pulvinar encode intentional hand movement and they have spatial preference. Only contralateral hand has been tested. Among those cells classified as "projection or arm movement related cells", some were stronger responding when both eye accompanied hand movement than either movement alone. Authors emphasized that these cells were active only during intentional movements but not passive movements. In addition, some of those projection neurons were more active under light- than dark-ambient condition, where monkey could have visual feedback of the hand. This suggests role of pulvinar in visually-guided arm movements or eye-hand coordination.

In the other two studies from the same lab (Acuna et al., 1990; Cudeiro et al., 1989), authors recorded neuronal (spiking) activities in the lateral posterior and pulvinar as well as parietal cortical area $5 \mathrm{~d}$ of four Macaca nemestrina monkeys. They trained monkeys to perform goal-directed arm movements to four spatial directions. Since monkeys had to fixate the upcoming target, the spatial selectivity of peripheral cue responses could not be tested. They reported that only $16 \%$ of total recorded cells were responsive to reach. Among all those reach-related cells, 77\% show no directional specificity and only $23 \%$ were spatially tuned. They also asked whether cells in lateral posterior 
pulvinar (lp-Pulv) are responsive to ipsi- or contralateral hand differently (hand-specificity responses). Interestingly all cells showed preference for contralateral hand. In addition, they reported that cells' responses were not modulated significantly by initial hand position and movement amplitude. Six cells showed pre-reach responses (Cudeiro et al., 1989), further suggesting possible contribution of pulvinar to motor programming. In contrast to thalamus, they found $75 \%$ of cells recorded in area $5 \mathrm{~d}$ as reach related and $76 \%$ of those were directional selective. Their data suggest that cells in lateral-posterior and pulvinar thalamus, which have reciprocal connections with frontoparietal reach related areas (Cappe et al., 2009, 2007; Yeterian \& Pandya, 1985), may involve functionally to reach planning and execution. In general, these studies show that neurons in pulvinar also encode arm movements similar to frontoparietal areas.

\subsubsection{Aims}

To the best of our knowledge, here we address for the first time hand-space representations in the local field potential oscillations in dorsal pulvinar (dPul) compared to area MIP in macaques. Toward this aim we trained two monkeys to perform three visually-guided tasks: dissociated delay saccade, dissociated delay reach (extra-foveal) and free-gaze delay reach (foveal). Monkeys performed saccades and reaches with either ipsi- or contralateral arm to ipsi-or contralateral hemifield relative to the recorded hemisphere. While monkeys were performing these tasks in blocks, we recorded local field potentials and spiking activity in area MIP and pulvinar (not simultaneously) from two hemispheres in one monkey and one hemisphere in another monkey. This chapter is focusing on the analysis of LFPs.

We ask explicitly how effector-specific (eye vs. left hand vs right hand), and task-specific (dissociated saccade, dissociated reach, free-gaze (mostly coordinated) reach) is LFP in dorsal pulvinar compared to MIP. We also ask to what extent LFP signals are spatially tuned, and whether this potential tuning is different in the dorsal pulvinar as compared to MIP. In addition, we compared these representations between more posterior LFP sites (partially covering area PRR) with those which are more anterior.

\subsection{Methods}

\subsubsection{Procedures}

All experimental procedures were conducted in accordance with the European Directive 2010/63/EU, the corresponding German law governing animal welfare, and German Primate Center institutional guidelines. The procedures were approved by the responsible government agency (Niedersaechsisches Landesamt fuer Verbraucherschutz und Lebensmittelsicherheit (LAVES), Oldenburg, Germany).

\subsubsection{Animal preparation}

Two male rhesus monkeys (Macaca mulatta), monkey L weighing $\sim 10 \mathrm{~kg}$ and monkey $\mathrm{T}$ weighing $\sim 7 \mathrm{~kg}$ served as subjects. In an initial surgery, the animal was implanted with an MRI-compatible polyetheretherketone (PEEK) headpost embedded in a bone cement headcap (Palacos with gentamicin; BioMet) anchored by ceramic screws (Rogue Research) under general anesthesia and aseptic 
conditions. MR-visible markers were embedded in the head cap to aid the planning of the chamber in stereotaxic space with the MR-guided stereotaxic navigation software Planner (Ohayon \& Tsao, 2012). A separate surgery was performed to implant PEEK MRI-compatible chambers (inside diameter $22 \mathrm{~mm}$ ) allowing access to the pulvinar and medial and lateral intraparietal areas (MIP and LIP respectively). After confirming chamber positioning with a postsurgical MRI, a partial craniotomy was made inside the chambers.

\subsubsection{MR imaging}

The monkeys were scanned in a 3 T MRI scanner (Magnetom TIM Trio; Siemens). Full-head T1-weighted (3D magnetization-prepared rapid gradient-echo, MPRAGE, $0.5 \mathrm{~mm}$ isometric) and additional T2-weighted (rapid acquisition with relaxation enhancement, RARE, $0.25 \mathrm{~mm}$ in plane, $1 \mathrm{~mm}$ slice thickness) images with the slice package aligned to the chamber vertical axis were acquired before and after chamber implantation using the built-in gradient body transmit coil and a custom single-loop receive coil (Windmiller Kolster Scientific). In addition to preimplantation and postimplantation scans, similar T1- and T2-weighted scans were acquired periodically during the course of experiments either in an awake or sedated state to confirm electrode positioning. T1- and T2-weighted scans were coregistered and transformed into "chamber normal" (aligned to the chamber vertical axis) and to AP-PC space for electrode targeting and visualization. These images were acquired with the chamber and the grid (custom-made MR-compatible polyetherimide (Ultem)) filled with gadolinium (Magnevist; Bayer)/saline solution (proportion 1:200) with tungsten rods inserted in predefined grid locations for alignment purposes.

\subsubsection{Pulvinar and parietal targeting}

The location of the electrodes was estimated for every recording site based on anatomical MRI. Custom-made MR-compatible polyetherimide (Ultem) grids ( $0.8 \mathrm{~mm}$ hole spacing, $0.45 \mathrm{~mm}$ hole diameter) were used to position electrodes in the corresponding grid hole and estimated depth. During the penetration, recording electrodes were protected using a custom-made stainless steel guide tube (450 $\mu \mathrm{m}$ outer diameter, 27 gauge Spinocan, Braun Melsungen) which was attached to the Thomas Recording Mini Matrix 5 channel drive. The tip of the guide tube aimed to advance just below the dura according to the MRI images. We collected neuronal activity from medial bank of posterior parietal area (MIP) and in separate sessions from subcortical thalamic nucleus dorsal pulvinar either uni- or bihemispherically from monkey L (right MIP: 25 sessions, 4 grid-holes; left MIP: 25 sessions, 3 grid-holes; right Pul: 17 sessions, 2 grid-holes; right Pul: 17 sessions, 2 grid-holes) and only unihemispherically (left) in monkey T (MIP: 42 sessions, 9 grid-holes; Pul: 8 sessions, 2 grid-holes). Fig. 2.1 shows the anatomical locations of the recording grid-holes along the intraparietal sulcus (IPS) and also thalamic nucleus pulvinar for each monkey and each hemisphere. 


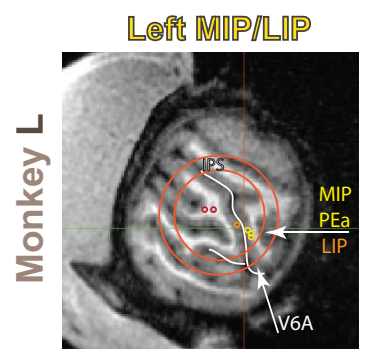

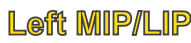

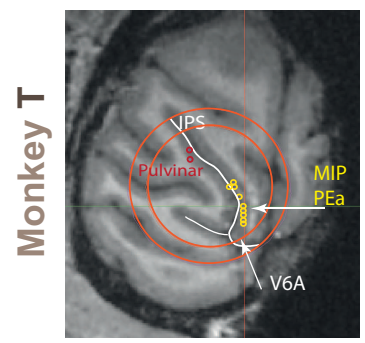

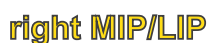
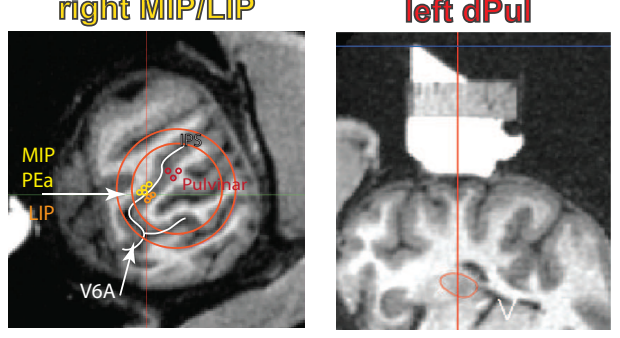

left dPul

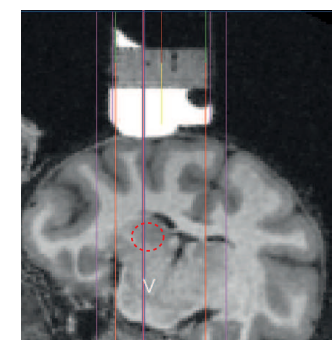

right dPul

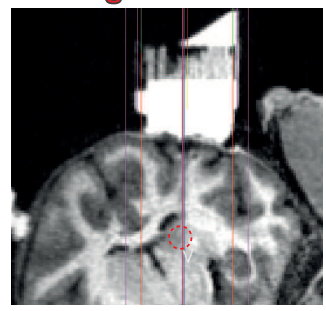

Fig. 2.1 Recording sites from parietal areas MIP (yellow grid-holes) as well as dorsal pulvinar (red grid-holes). We also recorded data from lateral intraparietal area (LIP; orange grid-holes) which will be discussed in chapter 3.

\subsubsection{Electrophysiological recordings}

Broadband neuronal activity was recorded with up to five individually movable single platinumtungsten (95-5\%) quartz glass-insulated electrodes with impedance ranging from 1 to $\mathrm{M} \Omega$ using a chamber-mounted five-channel Mini Matrix microdrive (Thomas Recording). Single custom-made stainless steel guide tubes (27 gauge) filled with the silicone oil (Thomas Recording) with a Spinocan funnel attached to the drive nozzle were used to protect electrodes during dura penetration. While recording, a reference tungsten rod or a silver wire were placed in the chamber filled with saline and were connected to the chassis of the drive. Neuronal signals were amplified (20x headstage, Thomas Recording; 5x, 128 or 32 channel PZ2 preamplifier, Tucker-Davis Technologies), digitized at $24 \mathrm{kHz}$ and 16 bit resolution, and sent via fiber optics to an RZ2 BioAmp Processor (Tucker-Davis Technologies) for online filtering, display, and storage on a hard drive together with behavioral and timing data streams. An offline procedure was used to obtain single/multi unit spiking activity as follows: raw broadband data was filtered through high-pass $(333 \mathrm{~Hz})$ and low-pass $(5000 \mathrm{~Hz})$ Butterworth filter (Matlab "butter" and "filtfilt" function).

To detect individual spikes, we applied a threshold $(3 *$ sigma $)$, where Sigma $=$ median(abs(signal))/0.6745 to the filtered broadband data of each recording site channel individually, meaning that sigma reached different values for different sites channels. Spikes that were detected within $1 \mathrm{~ms}$ of another, larger spike were discarded to avoid counting the same spike multiple times in case it crossed the threshold several times within one millisecond. Before clustering, we removed all spikes happened in the inter-trial-interval and also in the very beginning of each trial in which monkeys could do task task-unspecific movements. Feature detection and the clustering of the spike waveforms were done by the modified version of the offline sorter Wave Clus (Kraskov et al., 2009; Michaels et al., 2015; Quiroga et al., 2004). The procedure includes computing multiple features by means of different methods including "wavelet decomposition", "Principle component 
analysis (PCA)", " raw spike waveforms" and "1st order derivative of the spike waveforms" and then select the first best 11 features with highest non-unimodality distribution using Lilliefors test statistics ("Lillitest" in Matlab). Then superparamagnetic spike clustering was done on the selected features of a random sample of maximum 30000 spikes by applying Monte Carlo simulations with different starting values (see (Blatt et al., 1996)). In case there were more than 30000 detected spikes, the remaining spikes were clustered by template matching with the mean features of each cluster. This procedure was done of for the all data recorded within one recording site/channel, which could include several blocks of trials. This helped us to monitor the stability of spiking activity across time. The final control and resorting was applied using Plexon offline sorter (Plexon Inc, v3.0). To obtain LFP signal, we applied a median filter on the broad band signal with the window size of $250 \mathrm{~ms}$ which reliably gave us LFP signal for the frequencies up to $150 \mathrm{~Hz}$. To remove the $50 \mathrm{~Hz}$ AC line noise, band-stop Butterworth filter (Matlab "butter" and "filtfilt" functions) for the range of 49.9-50.1 Hz and also $99.9-100.1 \mathrm{~Hz}$ was applied.

\subsubsection{Behavioral paradigm}

The monkey was sitting in a dark room in a custom-made primate chair with the head restrained. Stimuli were presented on a 27" LED display (60 Hz refresh rate, model HN274H, Acer Inc. USA). The touchscreen was attached in front of the 27" stimuli display. The gaze position of the right eye was monitored at $220 \mathrm{~Hz}$ using an MCU02 ViewPoint infrared eyetracker (Arrington Research Inc. USA). The monkeys' jaw was monitored while they performed the tasks. Tasks were designed in MATLAB (version R2012b, The MathWorks, Inc., USA) using Psychophysics Toolbox. Both monkeys were trained to perform dissociated delayed response tasks in context of eye and hand movements (overall performance of $80 \%$ monkey L and $65 \%$ monkey T).

\section{Delayed response tasks}

All stimuli had circular shape with different size and color for different contingencies. Different colors served as signal for different effectors: red for eye, blue for left hand and green for the right hand. They were not exactly matching in luminance. Red stimuli had lower luminance than blue and green stimuli. The luminance of blue and green stimuli were almost similar. Monkeys learned to associate red color with the saccade, green with right and blue with left hand movements. Both monkeys learned to performed three different tasks Fig. 2.2: Delay dissociated reach (Ddr), delay dissociated saccade (Dds) and delay free-gaze reach (Ddf). In all of the tasks monkey started a trial by putting both hands on rest sensors mounted to the front part of the monkey chair. After a short time (500 ms), dim fixation stimuli ( $4^{\circ}$ radius for hand and on top of that $0.5^{\circ}$ radius for the eye) appeared on the middle of the screen (fixation acquisition period). As soon as monkey acquired the fixation spots with the eye and the required hand based on the color of the reach spot, they turned bright and monkey had to maintain fixation (fixation hold period; $500 \mathrm{~ms}$ in monkey L pulvinar dataset and in the rest 700-1200 ms). Then a single dim stimulus (instructed trials) or two dim stimuli in opposite directions relative to fixation spot (choice trials) appeared in the periphery (eccentricity of $24^{\circ}$ in monkey L pulvinar dataset and $16^{\circ}$ in the other datasets from the fixation spot; size $4^{\circ}$ radius). Monkey had to hold eye and hand fixations until one (in Ddr task, reach stimulus and in Dds task, 
eye stimulus) or both fixation stimuli (in Ddf task) disappeared (cue/delay period; 1000-1300 ms in monkey T, 1000-1500 ms in monkey L MIP dataset and $1280 \mathrm{~ms}$ in monkey L pulvinar dataset). This signaled monkey that he was allowed to begin the required movement (target acquisition period). Monkey had 700-800 ms to acquire a target (in monkey $\mathrm{T}$ for both eye and hand effectors but in monkey L $500 \mathrm{~ms}$ for eye and $1500 \mathrm{~ms}$ for hand effector). As soon as he acquired a target (first time that the required effector entered an allowed window around the target), period target hold started in which he kept the effector on the selected target for $500 \mathrm{~ms}$. Only after that (a successful trial) they received drops of juice as reward.

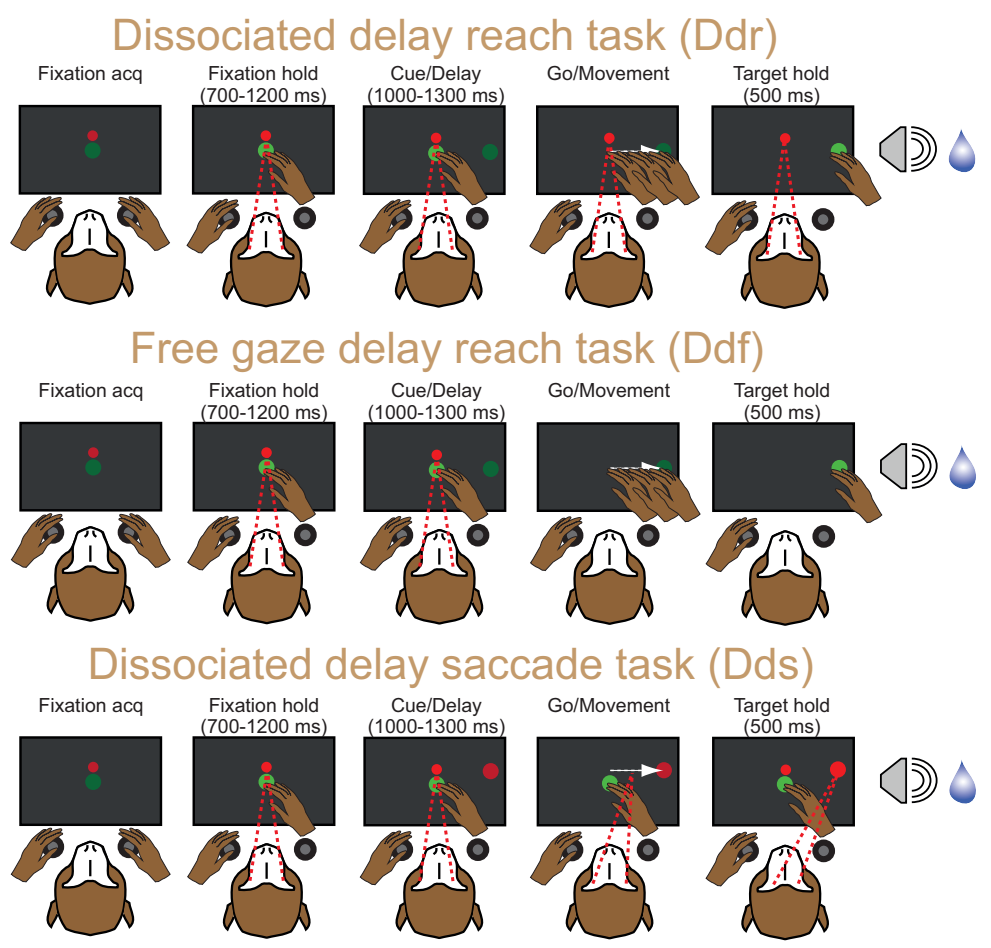

Fig. 2.2 Schematic of different tasks that monkeys performed in a block wise manner. a) Dissociated delay reach (Ddr) task: in this task monkeys fixated in the middle of the trial the whole time and executed arm movement either with right (green) or the left hand (blue). b) Delay free-gaze reach (Ddf) task: in this task monkeys kept fixation until target acquisition (Go) signal and during the arm movement eye was not constraint. c) Dissociated delay saccade (Dds) task: in which monkey kept one of the arms (based on the hand fixation color) in the middle of the screen the whole trial and executed eye movements. In each block of the trials (only one task) they performed movements to six different target locations (pseudo-randomized). Each block contained both instructed and choice trials (pseudo-randomized). The recording from pulvinar in both monkeys was done for only instructed trials.

Six target positions (in a circular geometry around the fixation spot) were used: $0^{\circ}, 40^{\circ}, 80^{\circ}, 140^{\circ}$, $180^{\circ}, 220^{\circ}$. Monkeys were allowed to acquire a stimulus within a window around it which slightly differed in different tasks: eye fixation/target window was $5^{\circ}$ radius and the hand fixation/target window was $4^{\circ}$; In the Dds task, eye window was set to $6^{\circ}$ or $7^{\circ}$ radius. Trials were aborted if the monkeys released incorrect hand from a sensor, didn't acquire stimuli within the limited time, didn't keep required holding times, moved the effector which had to keep fixating or moved their jaw during the course of a trial before reward. Each trial consists of several epochs: fixation acquisition (FixAcq), fixation hold (FixHld), Cue/delay (Cue/Del), target acquisition (TarAcq), target hold (TarHld), reward 
(Rwd). Most of the analysis reported in the next two chapters was done in the four main task epochs: fixation hold (600-100 ms before cue onset), cue (0-300 ms after cue onset), late delay (50-450 ms before go signal), movement (50-450 after movement onset).

\subsubsection{Data analysis}

All behavioral as well as neuronal signals were analyzed in MATLAB (versions R2011b, R2012b,R2014b The MathWorks, Inc., USA).

\section{Saccade definition}

Saccade velocity was calculated sample by sample as the square root of the sum of squared interpolated ( $220 \mathrm{~Hz}$ to $1 \mathrm{kHz}$ ), smoothed (12 ms moving average rectangular window) horizontal and vertical eye position traces, and then smoothed again (12 ms moving average rectangular window). Saccade onset was defined as eye position change that exceeded a starting velocity threshold $(300 \% \mathrm{~s})$ and the saccade offset as reaching an ending velocity $\left(50^{\circ} / \mathrm{s}\right)$ threshold.

\section{Reach definition}

Two types of reach latencies/reaction times (RT) and reach movement time (MT) were calculated: 1) Fixation: RT defined as the time between fixation spot onset and when the monkey released the required hand from the rest sensors (sensor release RT) and MT defined as the time between releasing hand and when the hand first touched inside the allowed window around the fixation spot (fixation MT). 2) Target: RT defined as the time between fixation spot(s) offset and the moment when the hand lost contact with the touch screen during the target acquisition period and MT defined as the time between the moment hand lost contact and the next contact with the touch screen. Reach inaccuracy was calculated as the mean reach offset (distance between the center of the target (or fixation) position and the actual position of the hand). Reach imprecision was calculated as the mean standard deviation of the reach offset.

\section{Local field potentials}

In total we recorded 204 LFP sites from MIP area in monkey L (108 left hemisphere and 96 right hemisphere) from which 92 sites participated in dissociated saccade (Dds), 165 sites in dissociated reach (Ddr) and 130 sites in free-gaze reach (Ddf). We recorded 146 sites from dPul in monkey L (104 from left and 42 from right hemisphere) from which 141 participated in Dds task, 136 in Ddr task and 141 in Ddf task. We also recorded in total, 191 LFP sites from left MIP in monkey T (107 sites with Dds, 143 with Ddr and 154 with Ddf task) and 31 LFP sites from his left dPul (22 sites with Dds, 21 with Ddr and 19 with Ddf task). Since monkeys did not perform in all sessions all three tasks but in some sessions only one or two tasks, we selected subsets of all recorded LFP sites to be able to compare the task-specific and region-specific hand-space representations. In addition, we rejected LFP sites which had more than 10 noisy trials per task condition.

LFP signal power and synchrony measures were computed using Fieldtrip toolbox (Oostenveld et al., 2011). LFP power was obtained for each of the frequency bins between 2 and $120 \mathrm{~Hz}$ in 
logarithmic steps ("logscale" function of MATLAB) by tapering data using "Hanning window" in a cycle-based time-window manner and then applying Fourier transform. To get the power time-frequency representation (TFR or spectrogram), we used a cycle-based time window for each frequency ( $\mathrm{N}=4$ cycles) with the step of $50 \mathrm{~ms}$ through one trial. This means for lower frequencies the time window was longer than for higher frequencies. Since for a typical length of trial (about 6 seconds), the full power distribution for the whole trial in lower frequencies was not possible, zero-padding was done such that the length of a trial was enough for power calculations for all frequency bins.

We also computed the average LFP power for separate frequency-bands (Delta [2 4) Hz, Theta [4 8) Hz, Alpha [8 12) Hz, Beta1 [12 18) Hz, Beta2 [18 30) Hz, Gamma1 [30 70) Hz, Gamma2 [70 120) $\mathrm{Hz}$ ), by averaging the power across all frequency bins within each band.

We normalized spectrograms within each session for the good of following reasons: to bring all frequencies to a comparable level (to account for $1 / \mathrm{f}$ nature of power spectrum) and also to be able to compare hand-space representations across blocks and sessions. Toward this, LFP power in each frequency was normalized by z-score approach (Ciuparu \& Mureşan, 2016): $P(f t)=(P(f t))-$ mean $(P)) / \operatorname{std}(P)$. The statistical measures (mean and standard deviation) were computed across all successful trials of the first Ddr task in a session (we took Ddr task as our reference for the comparisons). With this procedure we were able to investigate task selectivity as well as the handspace (condition) selectivity of the LFP power. We only analyzed a trial if it was not detected as noisy. To detect noisy trials we computed first the raw LFP power spectrogram and then mean and standard deviation of power across time-bins within each frequency. If at any time bin more than $50 \%$ of the frequency bins had power more than mean $(f))+2 * s t d(f)$, that trial detected as noisy. This is because we observed that spurious body movements caused sharp broad-band (across many frequencies) increase in power.

\section{Single/multi unit activity}

Spike density function for each trial was calculated using Fieldtrip toolbox by running a Gaussian kernel with a standard deviation of $25 \mathrm{~ms}$ for a window size of $100 \mathrm{~ms}$, sampled each $2 \mathrm{~ms}$.

\section{Statistical analysis}

To check the significant effect on individual LFP sites two-factor ANOVA was computed (factors: hand, space) separately for instructed and choice trials and separately for each of seven frequency band (as above) and each epoch (fixation hold (50 ms after fixation hold until $50 \mathrm{~ms}$ before cue onset), cue (50-350 ms after cue onset), delay (350-50 before Go), movement ( $25 \mathrm{~ms}$ after movement onset until $25 \mathrm{~ms}$ before movement offset)). As a result, for each of those cases we may have one of the nine following main effects of the ANOVA (for the moment we ignore the interaction outcome of ANOVA): no main effect (no), ipsi-hand(IH), contra-hand(CH), ipsi-space(IS), contra-space(CS), ipsi-hand and ipsi-space (IHIS), ipsi-hand and contra-space (IHCS), contra-hand and ipsi-space (CHIS), contra-hand and contra-space (CHCS).

Due to our normalization method and as expected we have up (positive) and down (negative) modulation of the power across conditions and also across injection blocks of trials. This makes 
the definition of tuning complex. We defined the tuning (as one the above main-effect categories) as following: we first applied two-factor ANOVA and if there was any main effect and for each we defined the tuning based on the higher absolute value of the averaged normalized power across trials in one condition against the other. For example, if there was a main effect of hand for one of the frequency-epoch cases and averaged normalized power across trials for contralesional hand had a higher absolute value than in the ipsilesional hand, we defined this as a CH tuned LFP site. Then to define if this site shows positive (up) or negative (down) modulation we just looked if the tuned condition (in this example, contralesional hand trials) has a positive or negative value. In summary, we will show how many LFP sites show those nine categories of main hand and/or space tuning and for each category how many shows up or down modulation.

To compare if there is significant difference between contra versus ipsi-lateral hand or space representations on the population level, we applied paired ttest across sites with FDR multiple comparison correction ((Benjamini \& Hochberg, 1995); matlab function "fdr_bh") to reject false discovery for each time-frequency representation (TFR). In each bin with a significant difference we show the actual normalized power value and in the rest the value set to zero. In other words, we show the strength of the power only for the bins with a significant difference. These differential TFRs are referred to contralaterality profiles or contralateral selectivity profiles (ICSd: contralateral minus ipsilateral space; ICSd: contralateral minus ipsilateral hand).

To compare power spectrograms between two tasks or areas, we performed the same analysis. We applied two-sample ttest on each bin across LFP sites for two conditions and used FDR method (False discovery rate) to adjust the $\mathrm{p}$-value for the significance level.

To reduce the complexity, we plotted differential profiles which are the averaged power across time-frequency bins for each frequency band and each epoch (in total a $7 * 5$ colormap matrix). The difference between two conditions was defined as the population differential profile. If the same sites contributed to the two conditions (e.g. when comparing across tasks), differential profile computed as mean across differences, otherwise it was computed as difference of means. To test if this population differential profile was significant in each epoch-frequency combination, we calculated ttest and marked the non-significant pixels with a circle. We used the following frequency bands: delta [2 4) Hz, theta $[4$ 8)Hz, alpha $[8$ 13)Hz, beta1 $[1318) \mathrm{Hz}$, beta2 [18 30)Hz, gamma1 [30 70)Hz, gamma2 [70 120) $\mathrm{Hz}$ and following epochs: fxh (500-0 ms before cue), cue (0-300 after cue), del (600-1000 ms after cue), tgo (400-0 ms before movement onset), mvm (0-400 after movement onset).

To observe also the differential profile on the single site level, we plot the z-scored power of each site in one case versus the other for each epoch and frequency separately (scatter plots). Here we mentioned the same statistics as in the differential profile plots.

\subsection{Results}

In the following sections we will show mainly how local field potential signal in reach-related cortical medial intraparietal area (MIP) and subcortical dorsal pulvinar (dPul) represent hand and space variables in the context of visually-guided delayed response tasks. At first we will show example responses of LFP signals to different behavioral task conditions. Then we will compare the population signals (averaged across multiple recorded sites) from separate brain regions while monkeys were 
performing tasks (task-encoding). Following that but only in one monkey, we investigate the taskencoding in two different anatomical portions (posterior versus anterior part) of the MIP region.

Fig. 2.3 shows LFP spectrograms (a) and single unit spiking activity (b) recorded from an example site in medial intraparietal area (MIP) in the right hemisphere of monkey L while the monkey performed dissociated delay reach task (Ddr). The trials are separated to four different hand-space conditions (ipsilateral hand ipsilateral space (IHIS); ipsilateral hand contralateral space (IHCS); contralateral hand ipsilateral space (CHIS); contralateral hand contralateral space (CHCS)). We calculated the normalized spectrograms and also firing rates (both aligned to cue presentation) per trial and then averaged them across successful trials (N:30) in each condition. Each solid vertical line shows onset of an epoch and the dashed lines represent mean and standard deviation of movement onsets across trials. We normalized each LFP site spectrograms using z-score method by the statistics of the Ddr task (see Methods). According to this normalization procedure, this LFP site (a) shows certain modulation in its power across time points which is also condition specific.

During the sensor release and moving toward the fixation spot, power in the lower frequency bands (delta and theta) and also high frequency range (gamma) increases while in beta band decreases. This modulation is more when the monkey uses the left (contralateral) hand (called as contralateral hand tuning). During fixation hold period in which monkey is waiting for spatial information the beta range power increases while low and high frequencies decrease. In this example channel, we observed an increase in the alpha-beta power after cue presentation in the left (contralateral) hemifield (called as contralateral spatial tuning). This is followed by a mild increase in the lower gamma range until the movement onset.

a) Example LFP site "Lin_20170713_Site_06" in area MIP right hemisphere monkey L
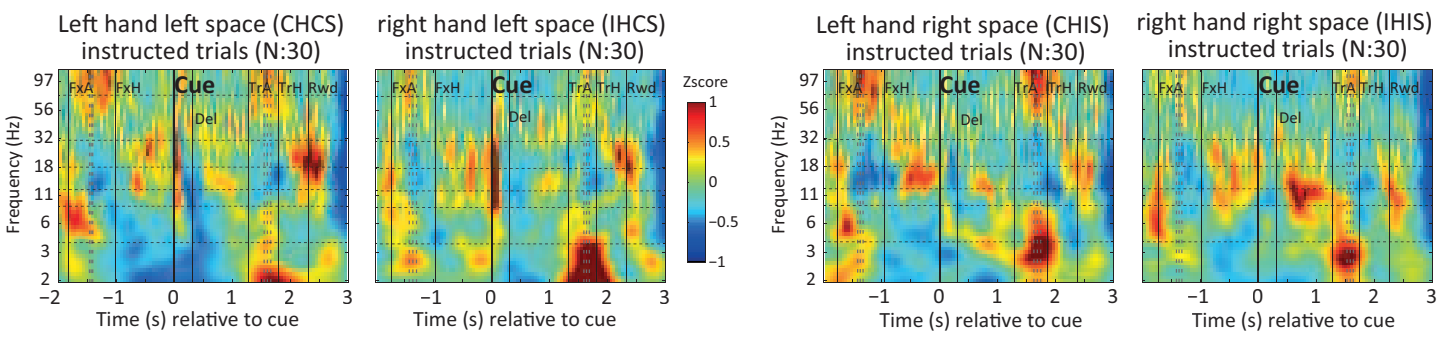

b) Example unit “Lin_20170713_10" recorded in the same channel and depth as (a)
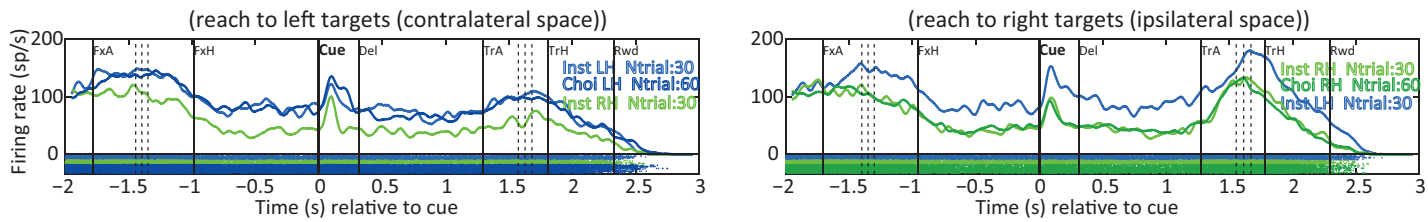

Fig. 2.3 (a) Local field potential spectrograms and (b) single unit activity (PSTH) recorded from an example channel landed in right hemisphere area MIP of monkey L. Monkey performed Dissociated delay reach (Ddr) task. Ipsi- and contralateral relative to the recorded hemisphere: ipsilateral hand ipsilateral space (IHIS); ipsilateral hand contralateral space (IHCS); contralateral hand ipsilateral space (CHIS); contralateral hand contralateral space (CHCS). Spectrograms are normalized using z-score method. Firing rates are not normalized. Both spectrograms and PSTHs are aligned to the cue presentation. Each panel of PSTH includes average firing rates on top and the raster of spikes on the bottom for each condition (blue: left hand, green: right hand, light: instructed, dark: choice). 
With the same recording channel and in the same location, we isolated a single-unit (b). This unit was active higher when the monkey performed the task with the left hand (contralateral) than the right hand. Besides that, it shows a typical task response: before (pre-) and during (peri-) movements and also just after cue presentation the firing rate increases and during the fixation hold and delay periods it fires at a constant level. It is also apparent that the firing for movement increases more toward the right side of space (ipsilateral) than the left space. As described in the Methods section, monkeys also performed bilateral target choice trials which were interleaved with instructed trials. This unit does not reflect any difference between these two trial types in its firing rate (dark (choice) versus bright (instructed) lines). Since in choice trials the monkey was selecting almost entirely left target with left hand and right targets with right hand, we see only green bold line in the right and blue bold in the left panel.

In the next sections we will show how the population neuronal signals on the level of local field potentials represent hand-space variables in the context of our different scientific questions.

\subsubsection{Encoding of hand-space variables in area MIP during dissociated delay reach task}

Fig. 2.4 and Fig. 2.5 illustrate the raw and normalized LFP power of the population of sites in the left hemisphere of monkey $\mathrm{L}$ and monkey $\mathrm{T}$, respectively.

Raw power spectrum has a general similar pattern across conditions within each monkey. In monkey L (Fig. 2.4(a)), high power in the lower frequency bands (delta and theta) follows by a decrease in the alpha band and has a sharp peak in the beta band. During fixation hold and late delay periods, power is higher than in the cue and movement periods. In contrast, gamma power (especially higher gamma) is higher during movement than other epochs. This pattern is slightly different in monkey T (Fig. 2.4(b)). Here increase of beta power is not as sharp as in monkey L and it spread through both low and high beta bands $(12-30 \mathrm{~Hz})$. In addition, and in contrast to monkey L, except the peak of beta power in fixation hold period, there is not a big difference between other epochs. And also gamma power is high for both delay and movement period in this monkey.

For a better investigation of task-related power modulations we looked at the normalized power across conditions. Here we not only looked at each condition separately (IHIS, IHCS, CHIS, CHCS) but also calculated the differences between conditions to check for the hand- and space-specificity (ICHd IS, ICHd CS, IH ICSd, CH ICSd). For that we applied paired ttest on each time-frequency bin and adjusted the p-value criterion for multiple comparisons based on the FDR method. In these plots, we assigned zero to the power for the non-significant bins.

Fig. 2.5(a) and Fig. 2.5(b) illustrate this analysis for monkey $\mathrm{L}$ and monkey T, respectively. In monkey $\mathrm{L}$, power in the lower beta band has a peak during entire fixation hold period in all conditions but in the theta and alpha bands only for ipsilateral hand. In this monkey we observe a sharp cue response for contralateral targets which covers theta to higher beta bands. This follows by the decrease of power in the same frequency bands in the early delay period and also increase of power up to the target acquisition time. As soon as the monkey starts preparing the movement (pre-movement) until the time that he finishes the movement power in the higher gamma increases and in the alpha and beta severely decreases. 
a) Averaged raw power spectrum across sites in left hemisphere MIP (monkey L)

Dissociated delay reach (Ddr) instructed trials (N:82)
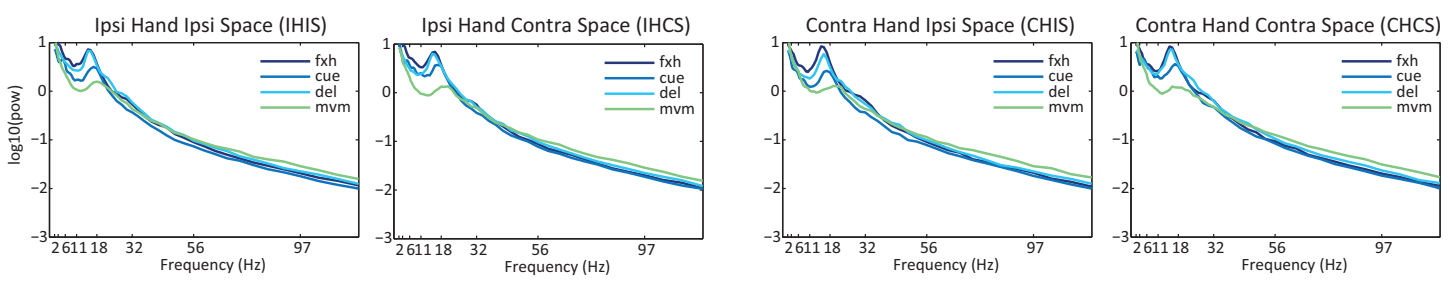

b) Averaged raw power spectrum across sites in left hemisphere MIP (monkey T)

Dissociated delay reach (Ddr) instructed trials ( $N: 44)$
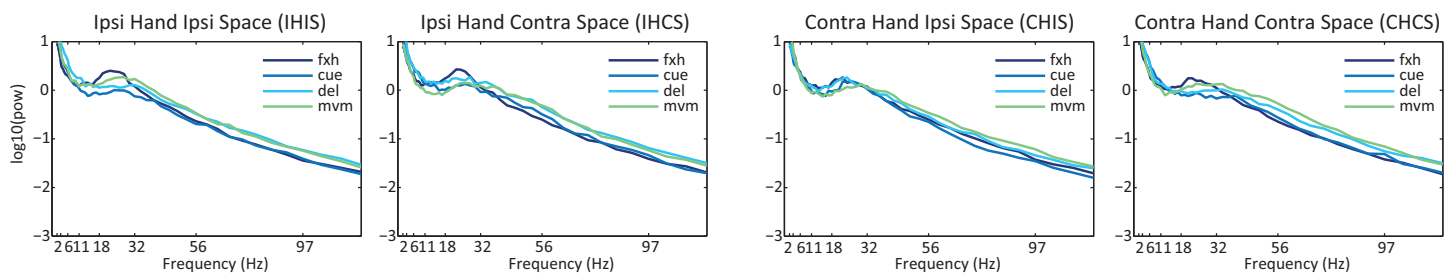

Fig. 2.4 Raw power spectrum (logarithmic scale) averaged across (a) 82 LFP sites recorded from left MIP in monkey L and (b) 42 sites in monkey T, while monkeys performed the instructed trials of dissociated delay reach task (Ddr). Each panel shows one task condition. Each color represents one epoch (fixation hold (fxh), cue, late delay (del) and peri-movement $(\mathrm{mvm}))$. Ipsi and contra relative to the recorded hemisphere.

These responses result in significant contralateral space-selectivity in theta, alpha and beta frequency bands which is present for both hands (corrected p-values: IHICSd 0.0191; CHICSd 0.0161). But we observe a mild contralateral hand-selectivity in the fixation hold period in the beta range and oppositely an ipsilateral hand-selectivity for the theta and alpha range (corrected p-value: ICHdIS 0.0270; ICHdCS 0.0272). Moreover, there is a mild contralateral hand-selectivity during the movement execution for both sides of space.

In monkey $\mathrm{T}$, we also see an increase in beta during fixation hold but compared to monkey $\mathrm{L}$, this is shifted to higher beta (beta2) band and it is significantly ipsilateral hand-selective (corrected p-values: ICHdIS 0.0267; ICHdCS 0.0206). But similar to monkey L, there is a sharp increase in theta-beta power after cue presentation. This results in a significant contralateral space-selectivity which is present for both hands (corrected p-values: IHICSd 0.0184; CHICSd 0.0250).

In addition, during the delay period, gamma power increases but only for the contralateral targets (significant contralateral space-selective). This follows by a significant increase in lower gamma power during the movement itself only for the contralateral space (significant contralateral space-selective). Only in monkey $\mathrm{T}$, beta power during delay period shows different patterns for each spatial target. While power for contralateral targets shows a mild but significant ipsilateral hand-selectivity, it has a strong significant contralateral hand-selectivity for ipsilateral targets.

Now that we illustrated the observed patterns of power spectrum in area MIP during the dissociated delay reach task, we ask how this cortical brain area represents other tasks with slightly different eye and hand fixation requirements. Three tasks were performed in separate blocks. The initial part of trial for all tasks (fixation up to cue presentation) was similar. The color of the cue signaled to the 
monkey whether the trial required a reach with left or right hand (blue vs green color) or required a saccade (red color) toward spatial target.

\subsubsection{How are different tasks represented in MIP MIP power spectrum in Ddr vs Dds}

Fig. 2.6 and Fig. 2.7 illustrate the normalized power spectrogram of LFP signal recorded from area MIP in Ddr (dissociated reach) task relative to Dds (dissociated saccade) task, in monkey L and monkey $\mathrm{T}$ respectively. As expected, beta power during fixation hold period shows overall nonsignificant differences between the two tasks. This is also happening for monkey T. But in monkey L, in the large number of bins we observe a significant higher power in the lower frequency bands in Dds task than Ddr. This pattern has a peak after the cue presentation. This peak is stronger while monkey performs ipsilateral arm movements as compared to contralateral. In addition, the entire delay period, alpha and beta range power is significantly lower in reaches than for saccades.

During pre-movement, gamma power is significantly higher for reaches than for saccades in all task conditions (FDR corrected p-values: IHIS 0.0312, IHCS 0.0277, CHIS 0.0328, CHCS 0.0269). In the same task period, theta, alpha and beta power is significantly lower for reaches than saccades.

Beside the general conditions (IHIS, IHCS, CHIS, CHCS), we do not see a strong significant differences between the two task in terms of the hand- and space-selectivity (FDR corrected p-values: IHICSd 0.0103, CHICSd 0.0126, ICHdIS 0.0096, ICHdCS 0.0041). One difference is that after cue presentation theta band power contralateral space- and hand-selectivity is higher for reaches than saccades. Besides that, the beta band contralateral hand-selectivity for ipsilateral targets during delay period up to movement onset is significantly higher for saccades than reaches. 
a) Averaged normalized power spectrogram across sites in left MIP (monkey L) N:82
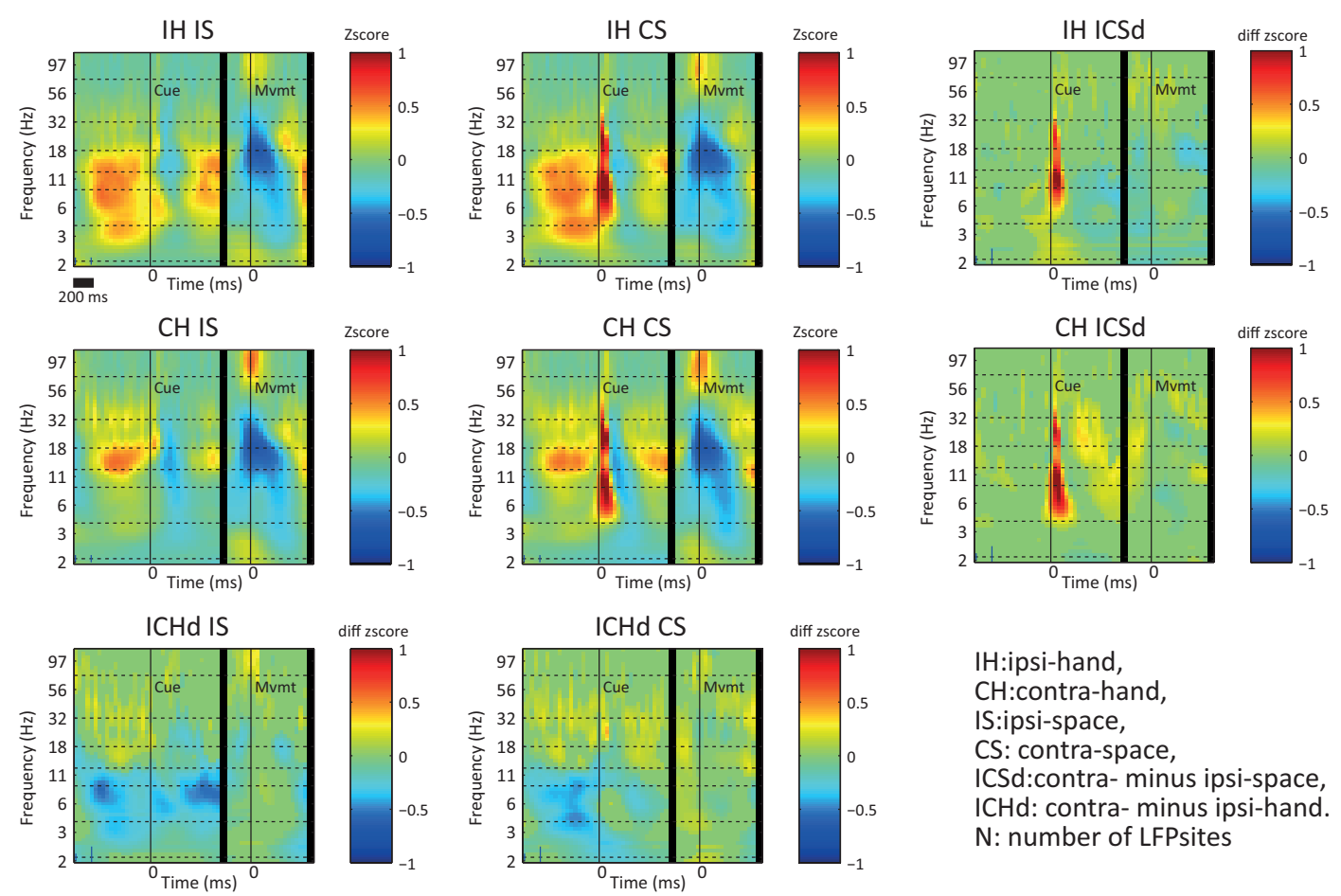
IH:ipsi-hand,
$\mathrm{CH}$ :contra-hand,
IS:ipsi-space,
CS: contra-space,
ICSd:contra- minus ipsi-space, ICHd: contra- minus ipsi-hand. $\mathrm{N}$ : number of LFPsites

b) Averaged normalized power spectrogram across sites in left MIP (monkey T) N:44
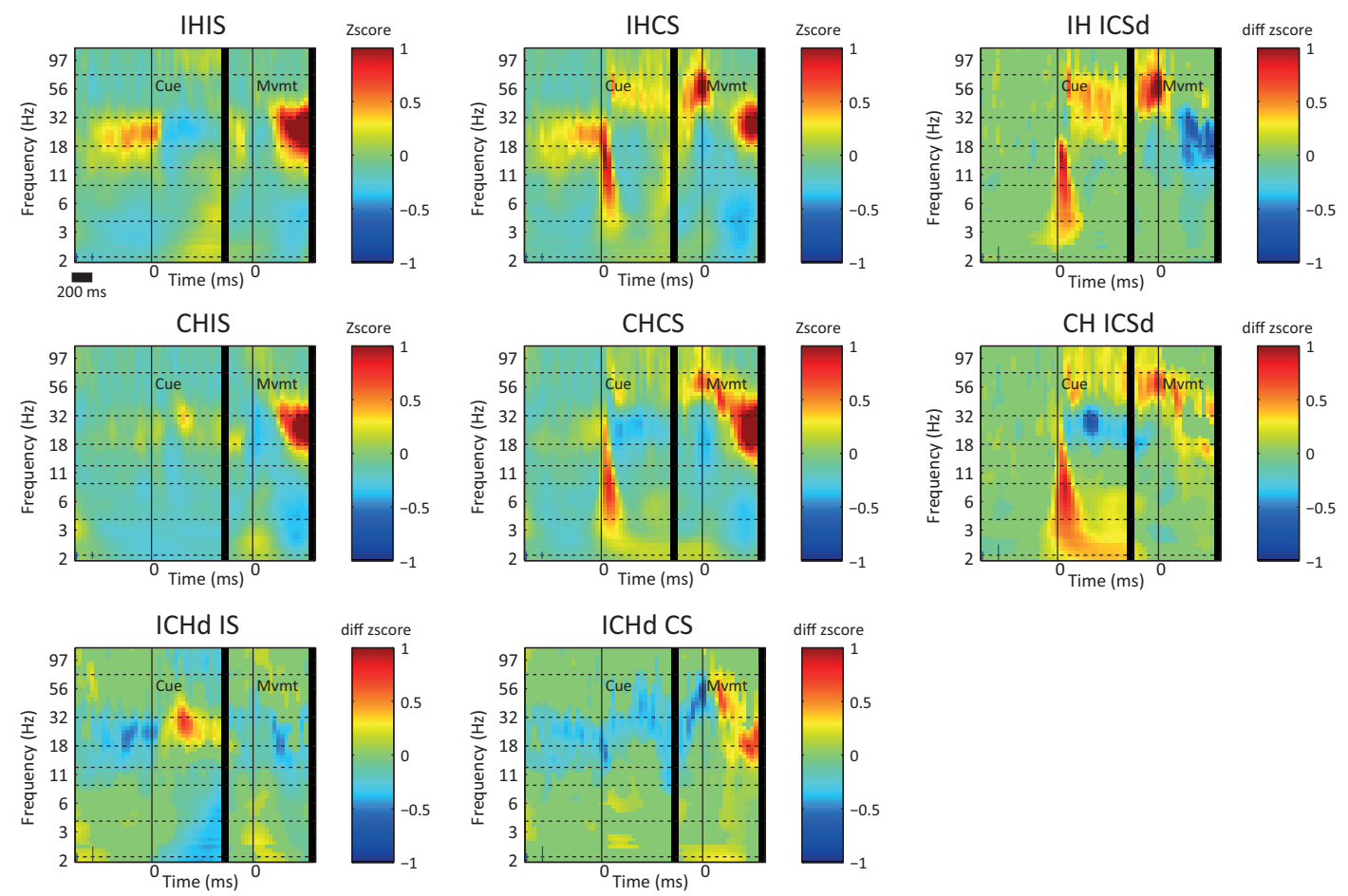

Fig. 2.5 Averaged z-scored spectrograms of the same data as in Fig. 2.4, aligned to cue and movement initiation (separated by the thick black vertical line). Horizontal dash lines represent seven frequency bands. Ipsi and contra relative to the recorded hemisphere. Condition abbreviations: IH (ipsi hand), CH (contra hand), IS (ipsi space), CS (contra space), ICSd (contra-space minus ipsi-space), ICSd(contra-hand minus ipsi-hand) 
Difference of normalized power spectrum in Ddr and Dds tasks across sites (N:36) in left hemisphere MIP (monkey L)
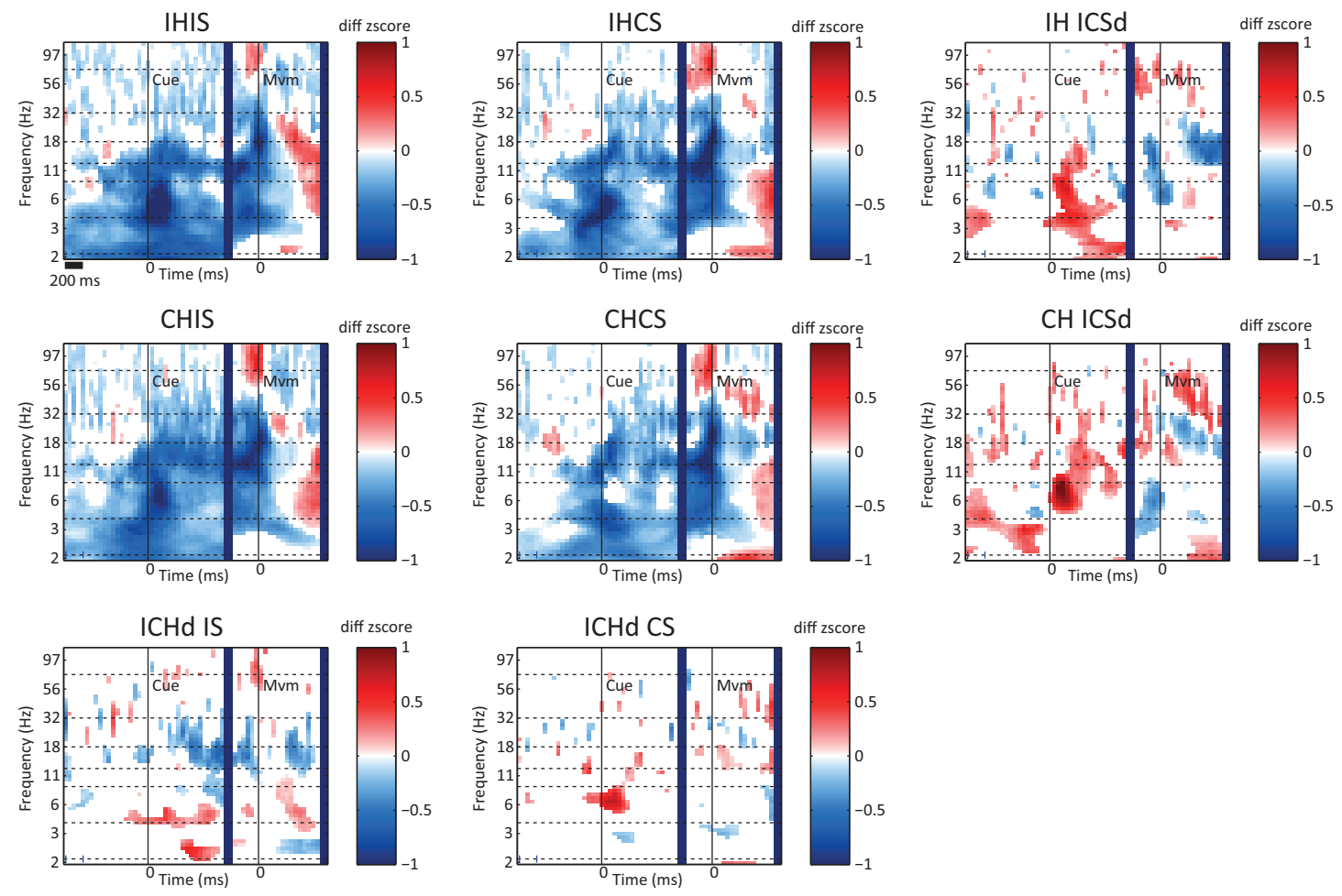

Fig. 2.6 Differential MIP normalized power spectrograms in different task conditions in dissociated reach (Ddr) relative to dissociated saccade (Dds) tasks (subtraction per LFP site and then averaged across sites; N=36). Each panel contains spectrograms aligned to cue and movement onset. Thick vertical lines are separators. Horizontal dashed lines separate our seven defined frequency bands. Paired ttest were applied on each time-frequency bin but p-value was corrected using the FDR method for multiple comparisons. Power of non-significant bins was set as zero (white). Ipsi and Contra are relative to the recorded hemisphere. Condition abbreviations: IH (ipsi hand), CH (contra hand), IS (ipsi space), CS (contra space). Note that the right column and the bottom row are differences of differences: ICSd (contra-space minus ipsi-space), ICSd (contra-hand minus ipsi-hand).

In monkey $\mathrm{T}$ similar to other monkey, there is a robust significant lower beta power during entire delay period in reach versus saccade task. This effect is much stronger when the ipsilateral hand is involved. During the movement and in all conditions except the IHCS, theta-alpha bands power is also significant higher for saccades than reaches (FDR corrected p-values: IHIS 0.0123, IHCS 0.0100 , CHIS 0.0112, CHCS 0.0078). Unlike in monkey L, we do not see any significant change in the lower frequency bands (delta, theta alpha) in the main conditions for monkey T. Main change, opposite to monkey L, is that beta power contralateral hand-selectivity is higher in Ddr than in Dds task. But similar to monkey L there is not a big change in the hand- and space-selectivity in most of the frequency bands (FDR corrected p-values: IHICSd 0.0063, CHICSd 0.0064, ICHdIS 0.0071, ICHdCS 0.0092). 
Difference of normalized power spectrum in Ddr and Dds tasks across sites (N:23) in left hemisphere MIP (monkey T)
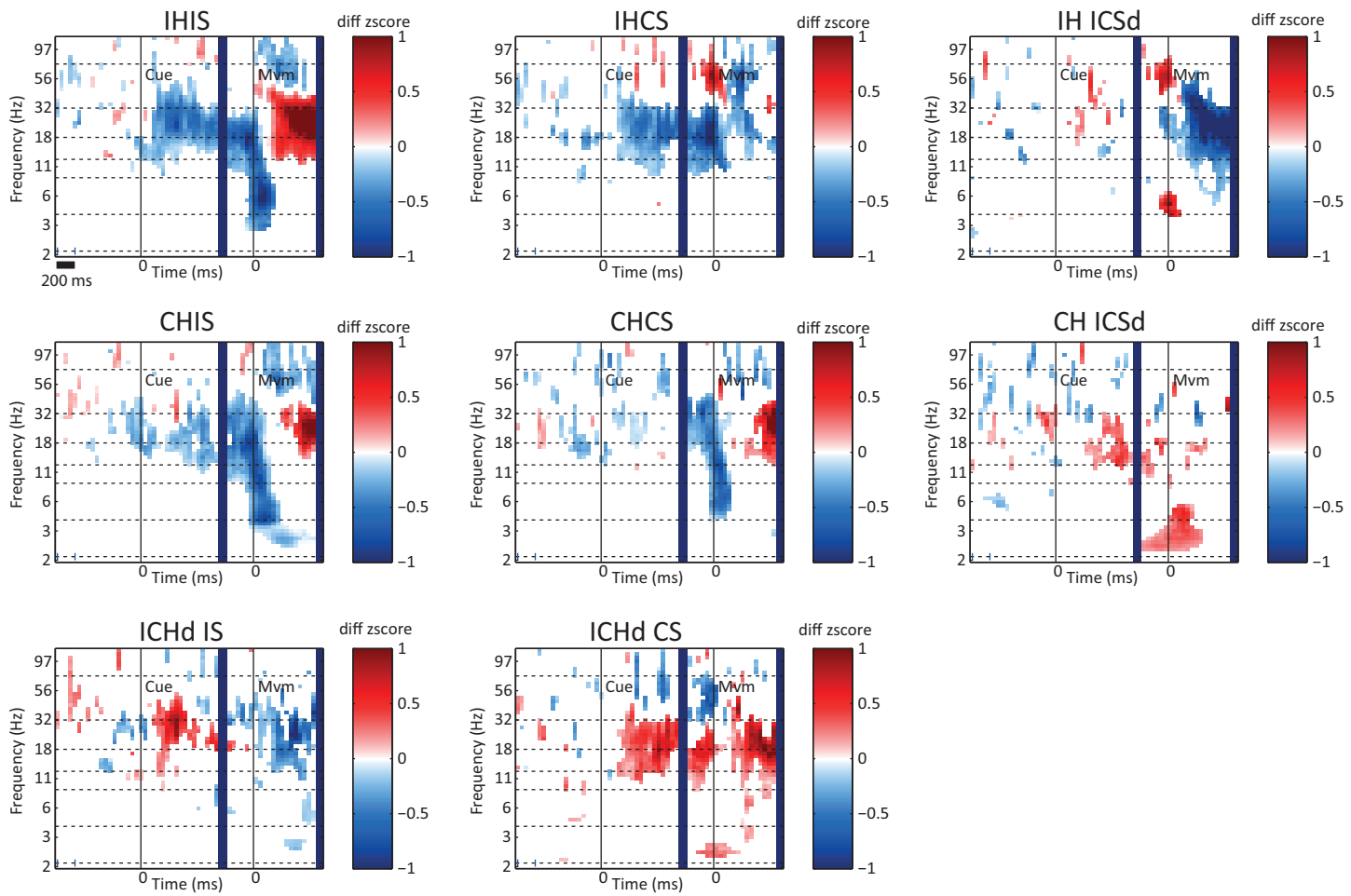

Fig. 2.7 Similar to Fig. 2.6 but for LFP sites recorded from MIP area of left hemisphere in monkey T (N=23)

\section{MIP power spectrum in Ddr vs Ddf}

In a separate block of trials, monkeys performed delayed free gaze reach (Ddf) task. Trials in this task were similar to Ddr task until the target acquisition time (tgo). But in Ddf task, after the target acquisition signal, eye movement was not constraint and monkey could look at the target. It is important to keep in mind that since task was performed in blocks, the monkey knew that he is going to do Ddf task after just a few trials.

Fig. 2.8 and Fig. 2.9 illustrate the normalized power spectrogram of LFP signal recorded from area MIP in Ddr task relative to Ddf task, in monkey $\mathrm{L}$ and monkey T respectively. The general difference is lower LFP power in the dissociated as compared to free-gaze reaches but in frequency-specific manner. But the frequency-epoch specific differences between tasks have some discrepancies across two monkeys. But for both monkeys the difference between dissociated reach and free-gaze reach is smaller than the difference between dissociated reach and saccade.

In monkey L, similar to the difference between Ddr and Dds task, power in the lower frequencies is significantly lower in the Ddr task than in Ddf before and after cue presentation (fixation hold, cue and early delay). This effect is stronger for ipsilateral hand (FDR corrected p-values: IHIS 0.0168, IHCS 0.0184, CHIS 0.0180, CHCS 0.0146).

Besides that but only in monkey $\mathrm{T}$, higher beta power during delay period is significantly lower for dissociated versus free-gaze reach, stronger for ipsilateral arm (FDR corrected p-values: IHIS 
0.0138, IHCS 0.0163, CHIS 0.0084, CHCS 0.0091). Another similarity between two monkeys is the lower theta-alpha power in Ddr task around the movement onset. This pattern is only pre-movement in monkey $\mathrm{L}$ but pre- and peri-movement for monkey $\mathrm{T}$. In addition, in monkey $\mathrm{L}$ pre-movement gamma band power is slightly but significantly lower for Ddr task than Ddf only for the ipsilateral targets. We see similar effect in monkey $\mathrm{T}$ peri- and post-movement periods.

\section{Difference of normalized power spectrum in Ddr and Ddf tasks} across sites (N:42) in left hemisphere MIP (monkey L)
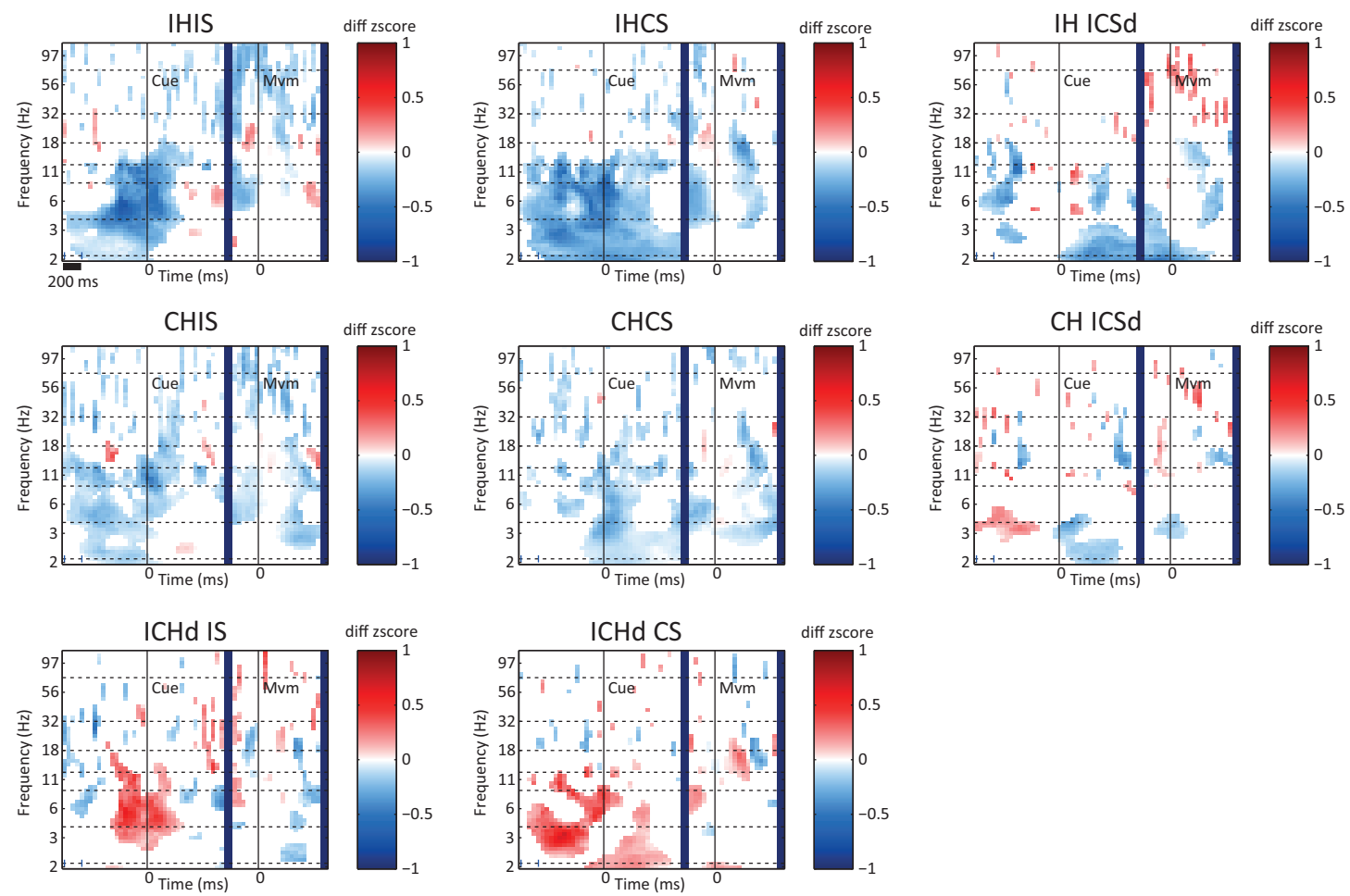

Fig. 2.8 Similar to Fig. 2.6. Here we compare Ddr task with Ddf task for the LFP sites recorded from left MIP of monkey $\mathrm{L}(\mathrm{N}: 42)$.

The overall hand- and space-selectivity does not differ much between the tasks. In monkey $\mathrm{L}$ and only delta-theta band power around the cue time show a significantly higher contralateral hand-selectivity in dissociated reach compared to free-gaze reach (FDR corrected p-values: IHICSd 0.0100, CHICSd 0.0064, ICHdIS 0.0098, ICHdCS 0.0089). This pattern is visible also in monkey T but only for the ipsilateral targets (FDR corrected p-values: IHICSd 0.0104, CHICSd 0.0075, ICHdIS 0.0094, ICHdCS 0.0099). In addition, beta power during delay period has a significantly higher contralateral hand-selectivity in Ddr task versus Ddf task only for the targets in contralateral space. 
Difference of normalized power spectrum in Ddr and Ddf tasks across sites (N:28) in left hemisphere MIP (monkey $\mathrm{T}$ )
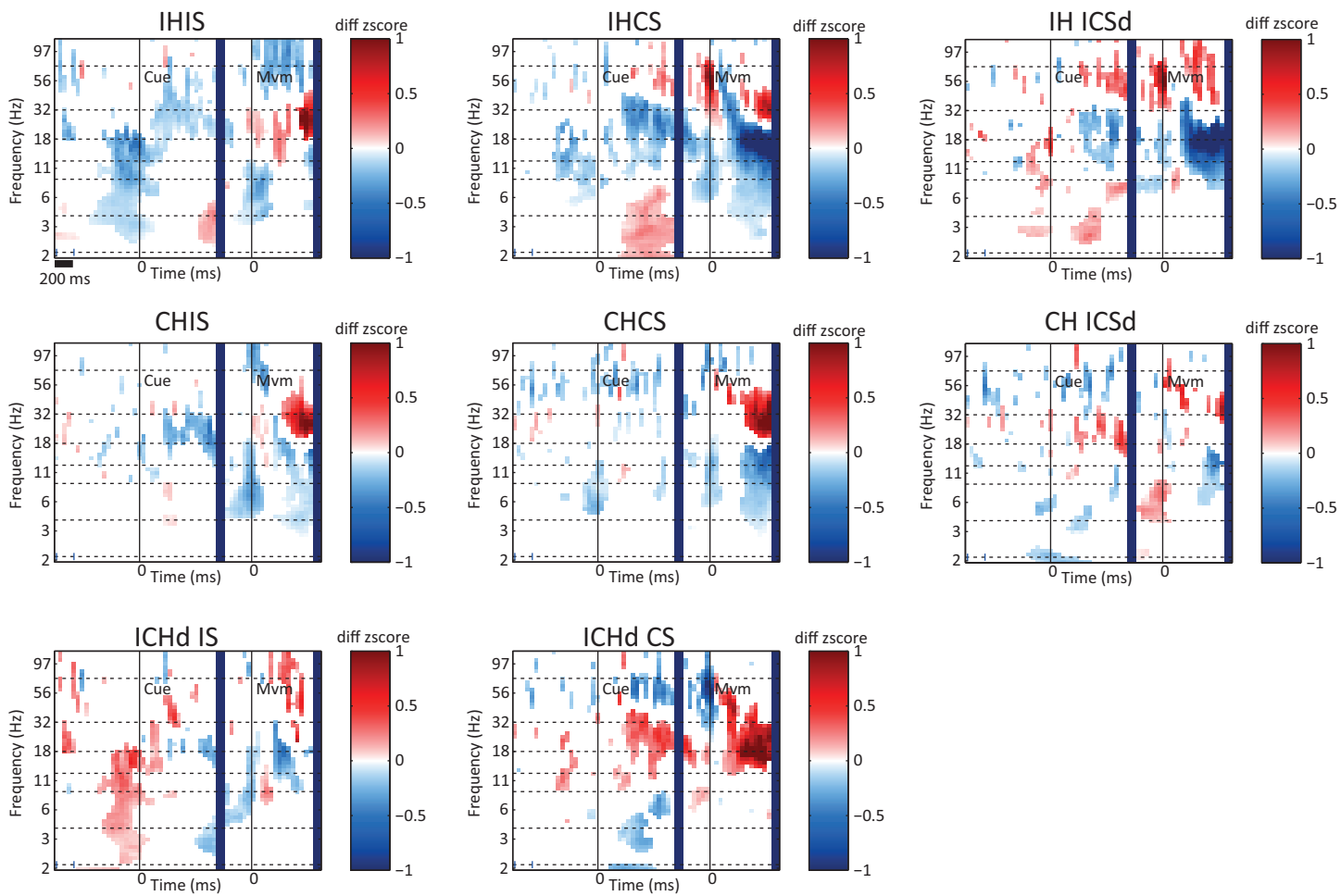

Fig. 2.9 Similar to Fig. 2.8 but for the LFP sites recorded from anterior part of MIP in left hemisphere of monkey T (N:28).

The question which comes following previous population analysis is that how consistent is the effect or difference between task representations across the population of LFP sites. Toward this we looked at the LFP power values in one task versus the other for each single LFP site. We averaged the normalized power in each of seven frequency bands and each of five epochs (fixation hold, cue, late delay, go and movement) and applied a paired ttest to check for significance. Figures 2.10 and 2.11 illustrate the scatter plot of normalized power in different epochs and frequencies for dissociated reach versus dissociated saccade respectively for monkey $\mathrm{L}$ and monkey $\mathrm{T}$.

In general, this analysis confirms the effects that we see on the spectrograms for both monkeys. In monkey $\mathrm{T}$ lower frequency (delta-theta) bands does not show significant differences between dissociated reach and saccade in almost any epochs and any condition (except in theta and only during movement period higher for saccades; paired ttest $\mathrm{df}=22 \mathrm{p}<0.012$ ). Also in the alpha range and mostly after go signal (tgo and mvm), we see higher power in saccades than reaches $(\mathrm{df}=22 \mathrm{p}<0.016)$. But in monkey $\mathrm{L}$ we see a highly significant higher power in all epochs and all conditions in delta-theta-alpha bands ( $\mathrm{p}<0.001$; except movement period in the delta range which is not significant in any condition).

Beta range power shows a similar pattern across monkeys. We see a significantly higher power during saccades versus reaches in all conditions in all epochs (fixation higher beta not significant in monkey L and partially significant in monkey $\mathrm{T}$ ). For most of the LFP sites power in the fixation lays very close along the diagonal which means that the power did not differ much across tasks (as seen on the spectrograms). 
High-gamma power is higher for reaches than saccades after go signal (pre-movement; $\mathrm{df}=35$, $\mathrm{p}<0.0001)$ in monkey L, and in monkey $\mathrm{T}$ only in CHIS condition $(\mathrm{df}=22 \mathrm{p}=0.017)$ and IHCS condition ( $\mathrm{df}=22 \mathrm{p}=0.003)$. Only in monkey $\mathrm{T}$ we see a significant lower high-gamma power in reach than saccade $(\mathrm{df}=22$; all conditions $\mathrm{p}<0.002)$. Figures 2.12 and 2.13 illustrate the scatter plot of normalized power in different epochs and frequencies for dissociated reach versus free gaze reach respectively for monkey $\mathrm{L}$ and monkey $\mathrm{T}$.

In both monkeys we see a tendency of higher power in free-gaze versus dissociated reaches in the lower frequency bands on the population level which is stronger for monkey $\mathrm{L}$ than monkey $\mathrm{T}$. The scatter of individual LFP sites in this frequency bands looks quite disperse although it shows significant differences in some epochs especially in monkey L (as also shown in the average spectrograms).

The inconsistency between two monkeys in the beta band power, which was noticeable from the comparison between averaged powers, is confirmed here (Fig. 2.7 compared to Fig. 2.8). While in monkey $\mathrm{T}$ beta band power is significantly lower for dissociated reach especially in the higher beta range (IHIS $p<0.02$, IHCS $p<0.001$, CHIS $p<0.04$ ), in monkey L it gets significant only for specific conditions (IHIS $\mathrm{p}<0.03$ and CHCS $\mathrm{p}<0.04$ ). This goes along the other effect that beta band power increases for saccades stronger in monkey $\mathrm{T}$ than in monkey $\mathrm{L}$.

So far we talked about the LFP hand-space representations in area MIP but we also did the similar analysis for the data collected in dorsal pulvinar. Later in this chapter we will show the comparison between MIP and pulvinar and how different they encode hand and space in separate epochs and frequency bands in different tasks. But before addressing that question we asked whether locations along the intraparietal sulcus that are closer to area V6A (in parieto-occipital sulcus, POS) are differently activated than more anterior locations while monkeys perform reach and saccade tasks. This question is important because it helps to understand whether even neighboring locations play different visuomotor processing roles. 
Dds vs Ddr task, instructed trials, monkey L left hemisphere ( $N: 36)$

Delta $[24) \mathrm{Hz}$
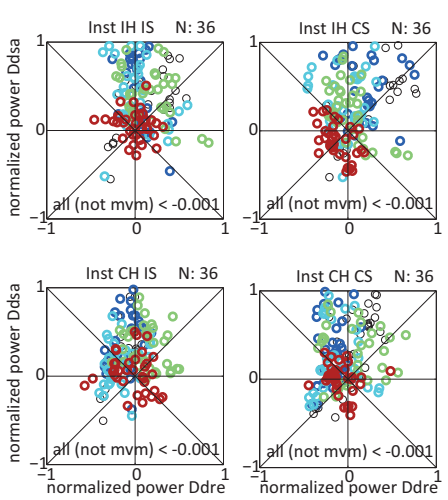

Beta1 $[11$ 18) $\mathrm{Hz}$
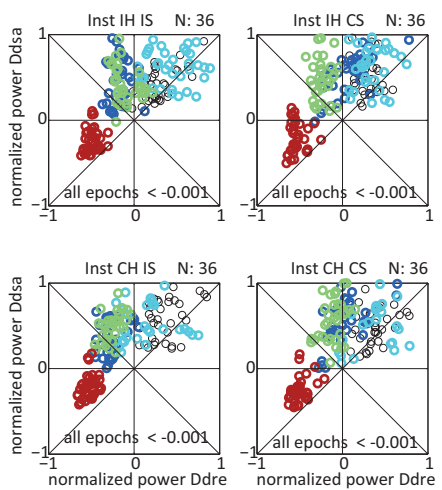

Low Gamma [30 70) Hz
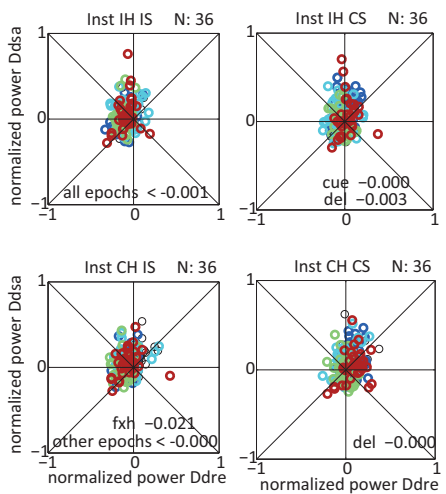

Theta $[48) \mathrm{Hz}$
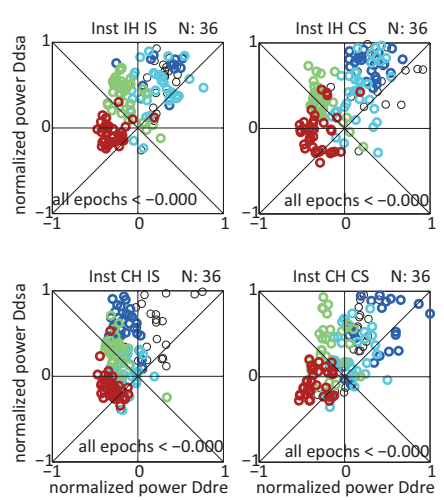

Beta2 $[1830) \mathrm{Hz}$
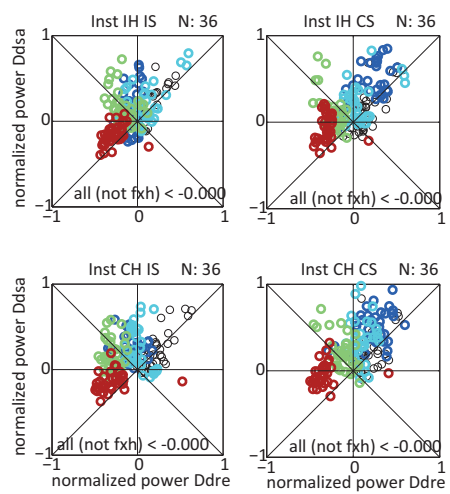

High Gamma [70 120)Hz
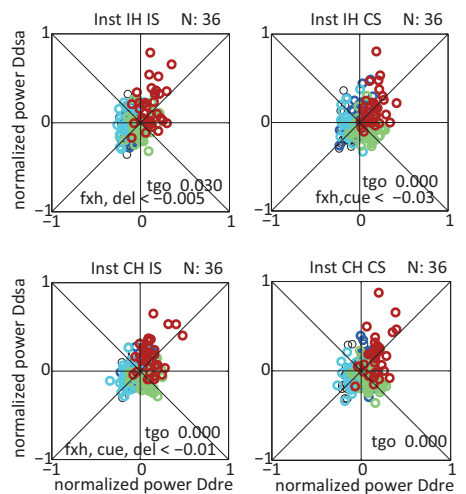

Alpha $[8$ 11) $\mathrm{Hz}$
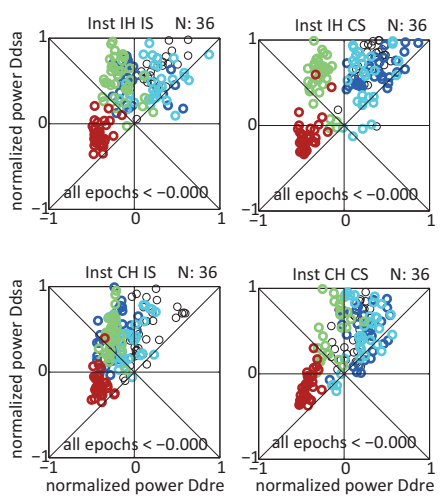

Fig. 2.10 Average normalized power in each epoch and frequency band for each individual LFP site in Ddr task (x-axis) versus Dds task (y-axis) (for population average differences see Fig. 2.6). For each frequency band there are four panels representing each task condition (IHIS, IHCS, CHIS, CHCS). In each panel colors represent trial epochs. Paired ttest were applied for the significant difference between tasks for each frequency band and each epoch across LFP sites. Those epochs which get significant are mentioned (p-value) in the panel. Negative p-values mean significant lower power in reach task. 
Dds vs Ddr task, instructed trials, monkey T left hemisphere (N:23)
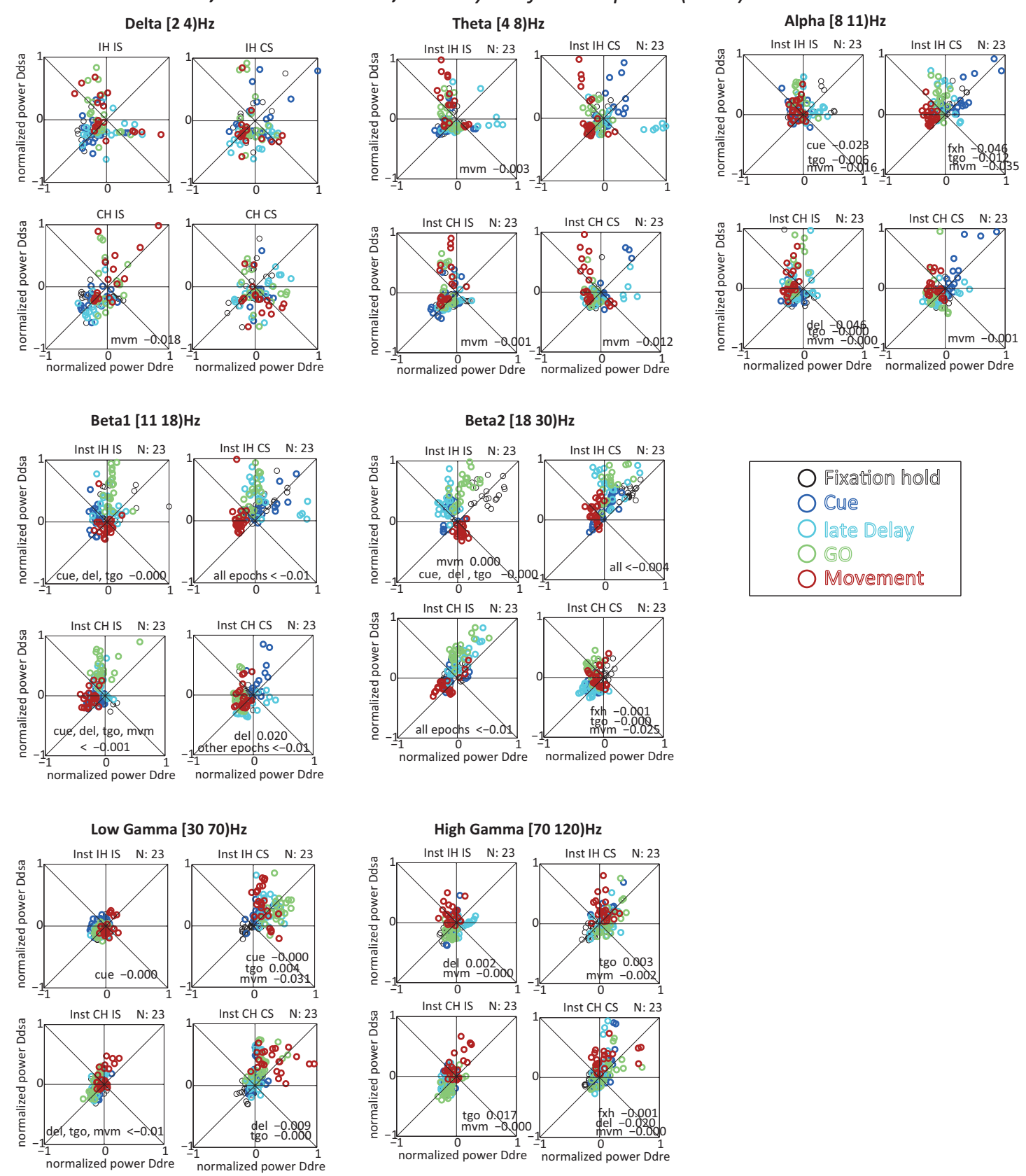

Fig. 2.11 Normalized power in each epoch and frequency band for each individual LFP site in Ddr task (x-axis) versus Dds task (y-axis) (for population average differences see Fig. 2.7). For each frequency band there are four panels representing each task condition (IHIS, IHCS, CHIS, CHCS). In each panel colors represent trial epochs. Paired ttest were applied for the significant difference between tasks for each frequency band and each epoch across LFP sites. Those epochs which get significant are mentioned ( $p$-value) in the panel. Negative p-values mean significant lower power in reach task. 
Ddf vs Ddr task, instructed trials, monkey L left hemisphere ( $N: 42)$
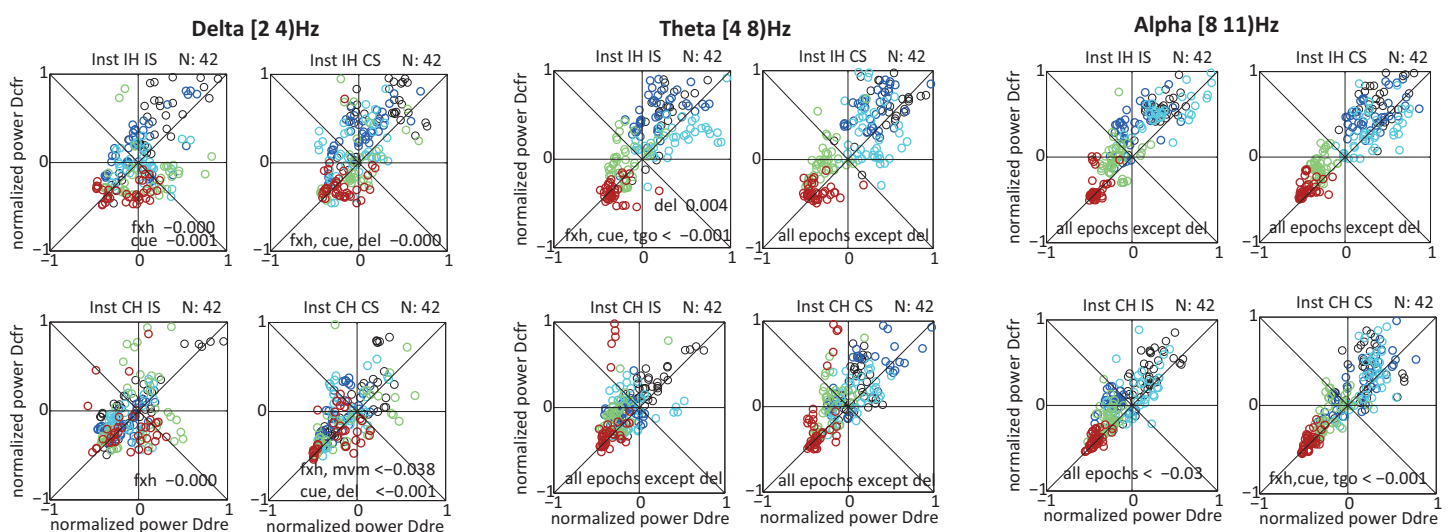

Beta1 [11 18) Hz
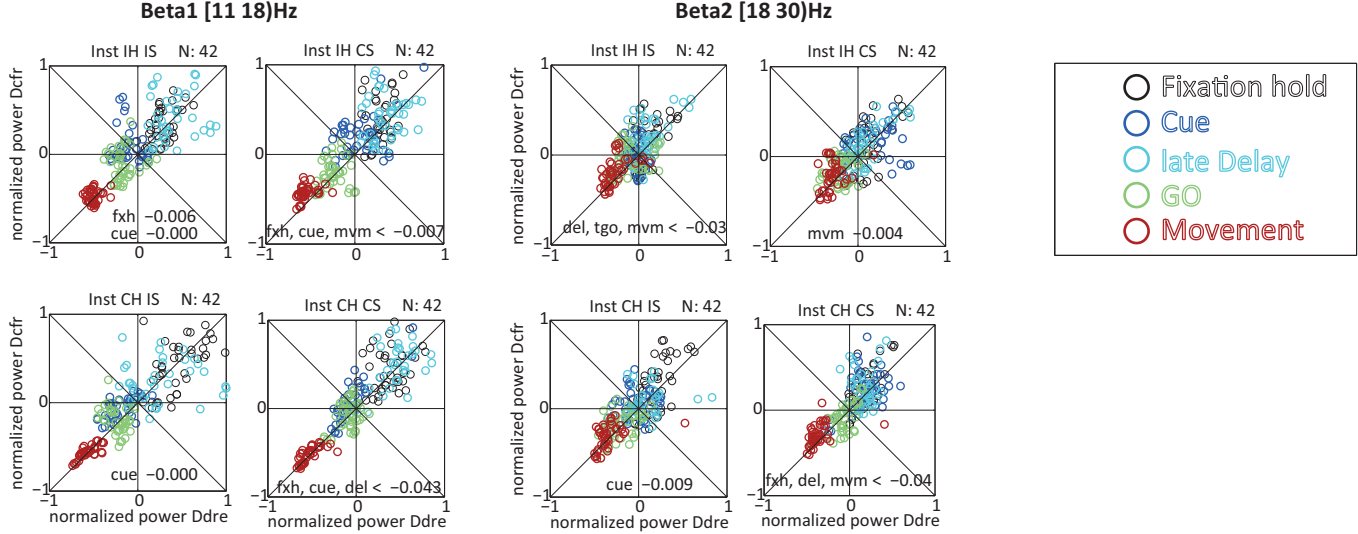

Low Gamma [30 70) Hz

High Gamma [70 120)Hz
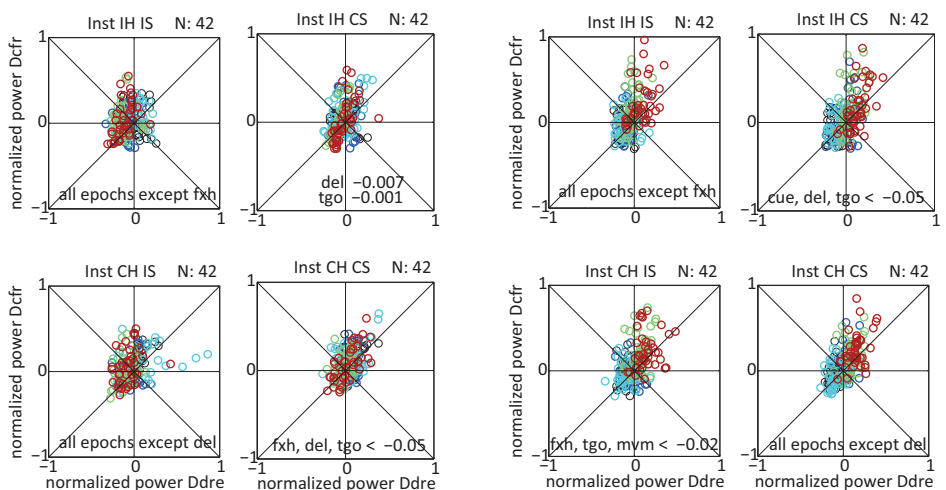

Fig. 2.12 Normalized power in each epoch and frequency band for each individual LFP site in Ddr task (x-axis) versus Ddf task (y-axis) (for population average differences see Fig. 2.8). For each frequency band there are four panels representing each task condition (IHIS, IHCS, CHIS, CHCS). In each panel colors represent trial epochs. Paired ttest were applied for the significant difference between tasks for each frequency band and each epoch across LFP sites. Those epochs which get significant are mentioned (p-value) in the panel. Negative p-values mean significant lower power in reach task. 
Ddf vs Ddr task, instructed trials, monkey T left hemisphere (N:28)
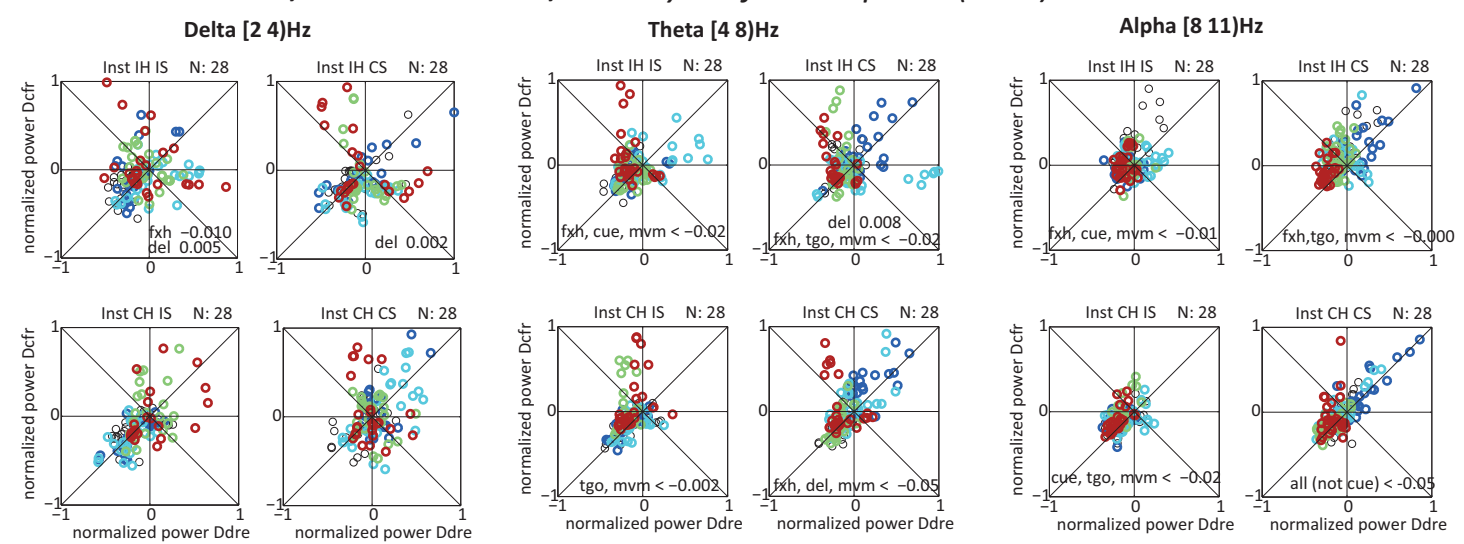

Beta1 $[11$ 18) Hz
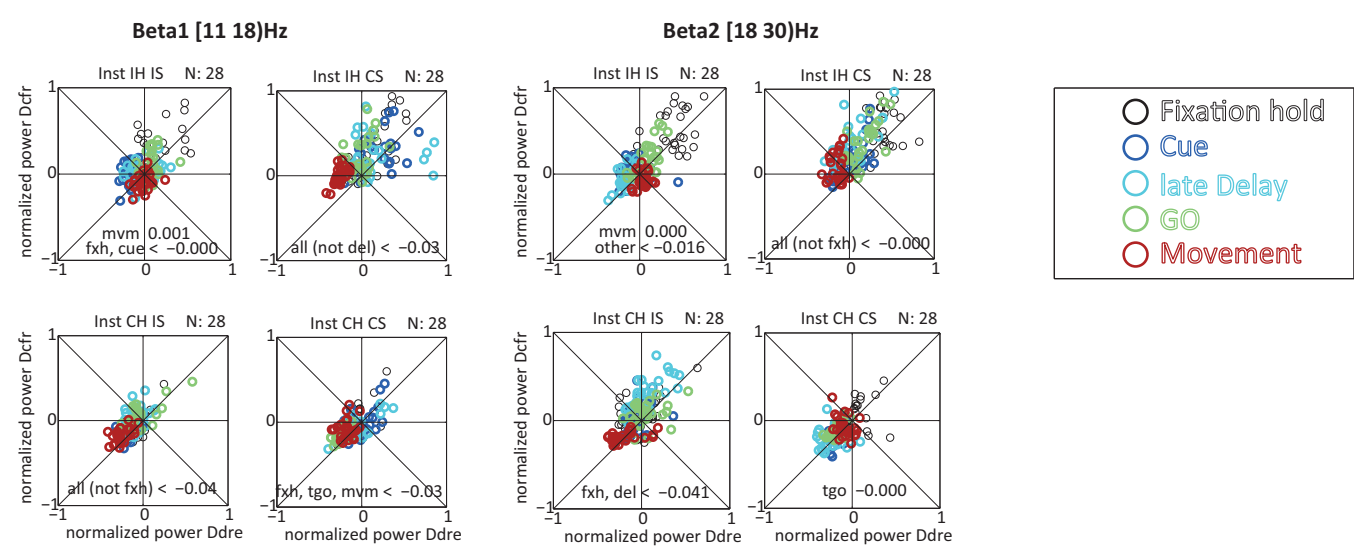

Low Gamma $[30$ 70)Hz

High Gamma [70 120)Hz
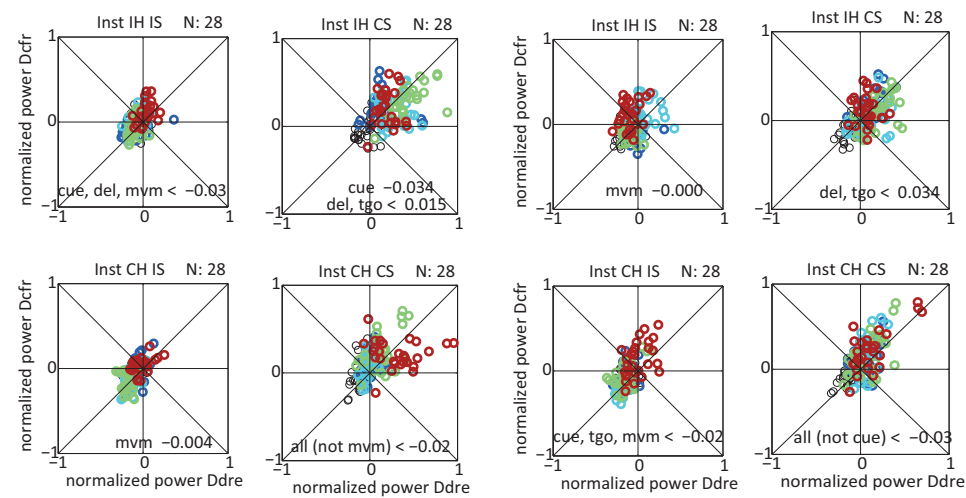

Fig. 2.13 Average normalized power in each epoch and frequency band for each individual LFP site in Ddr task (x-axis) versus Ddf task (y-axis) (for population average differences see Fig. 2.9). For each frequency band there are four panels representing each task condition (IHIS, IHCS, CHIS, CHCS). In each panel colors represent trial epochs. Paired ttest were applied for the significant difference between tasks for each frequency band and each epoch across LFP sites. Those epochs which get significant are mentioned (p-value) in the panel. Negative p-values mean significant lower power in reach task. 


\subsubsection{Are hand-space representations similar along IPS (posterior vs anterior loca- tions)?}

As mentioned in the Methods, we recorded neuronal activity from different locations in the medial bank along the intraparietal sulcus only in monkey $\mathrm{T}$ (See Fig. 2.1). These locations include posterior LFP sites which are closer to area V6A as well as more anterior LFP sites which covers more classical MIP. Here the question is whether hand-space coding during reach and saccade task changes when we move along the intraparietal sulcus. To address this question we compare the task specific response (reach vs saccade) of LFP power between those anatomical groups (40 LFP sites recorded from the posterior locations and 23 sites from anterior locations).

a) Averaged raw power spectrum across sites in posterior MIP (monkey T)

Dissociated delay reach (Ddr) instructed trials (N:56)
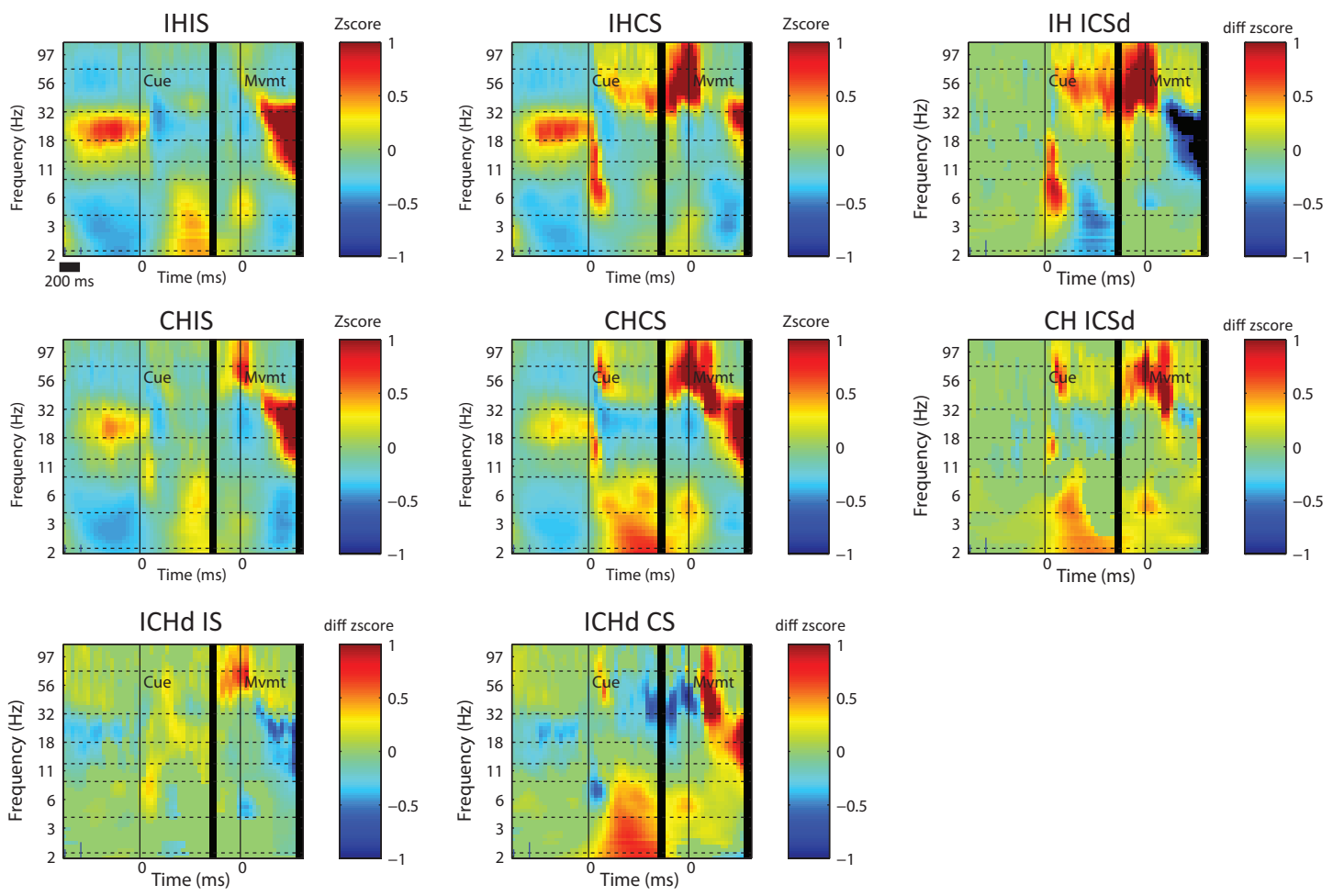

Fig. 2.14 Similar to Fig. 2.5(b) but for LFP sites recorded from posterior MIP while monkey T performed Ddr task.

First we show in Fig. 2.14 the averaged power spectrograms for the main task conditions and the differential TFRs (contralaterality profiles) for the LFP sites recorded in the posterior MIP in monkey $\mathrm{T}$ (similar to Fig. 2.5(b)). There is strong high beta band increase during entire fixation hold period which is stronger for ipsilateral than contralateral hand (ipsilateral hand tuning but no spatial preference). Cue presentation trigger brief theta-alpha band power increase for the targets in contralateral space (which is stronger for the ipsilateral hand). In addition, during the delay and movement preparation and execution, while beta band seems to stay unchanged (although compared to the fixation period there is a huge decrease), gamma band activity increases stronger for the contralateral than ipsilateral space (contralateral space preference). Theta band during movement 
increases slightly for the reaches with the hand ipsilateral to the spatial location of target (IHIS and CHCS).

Difference of normalized power spectrum in Ddr and Dds tasks across sites (N:40) in left hemisphere posterior MIP (monkey T)
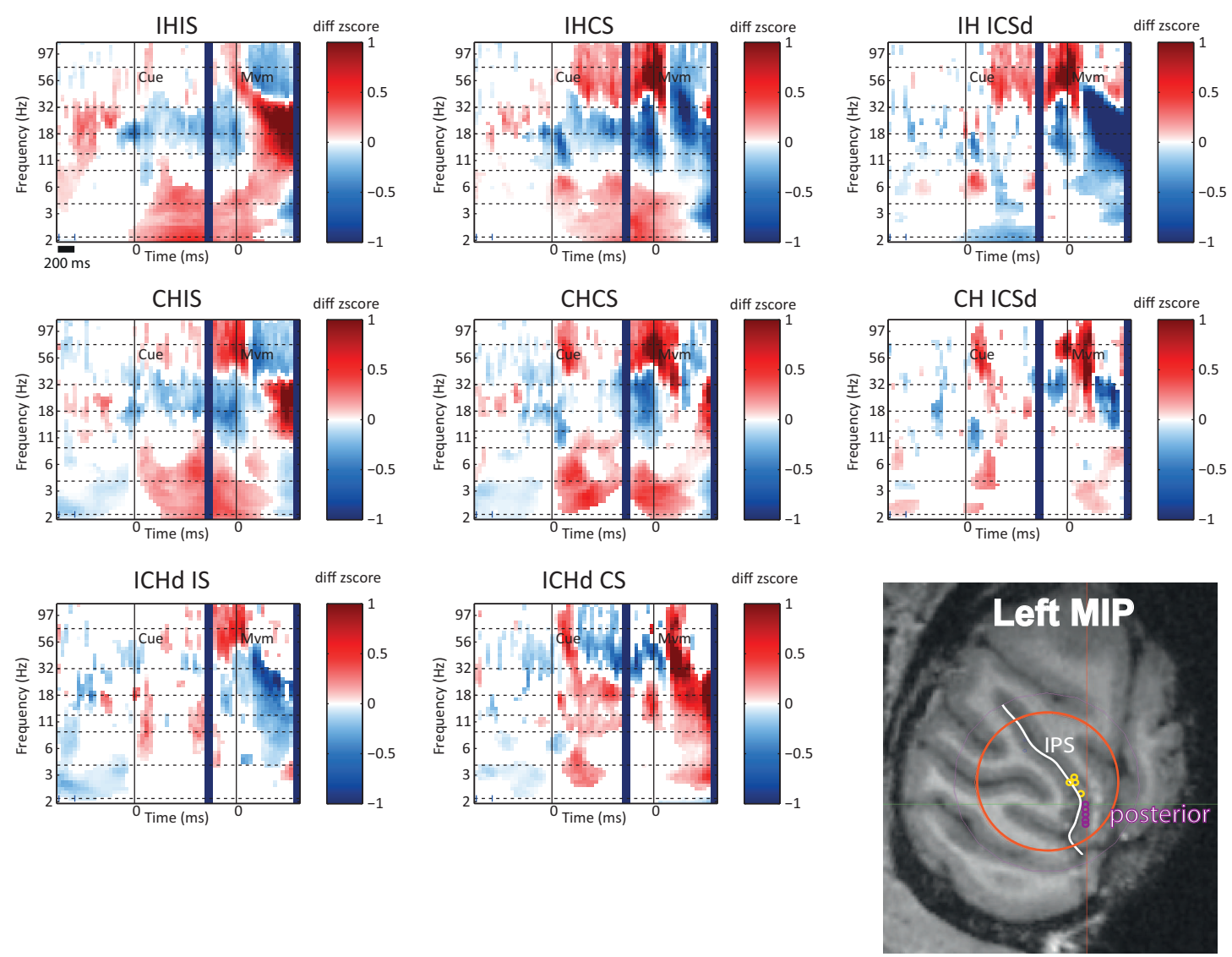

Fig. 2.15 Similar to Fig. 2.7 but for LFP sites recorded from posterior MIP. In the bottom right corner, we see the anatomical locations of the groups of LFP sites we chose for posterior (purple) and anterior (yellow) MIP.

Fig. 2.7 shows the averaged normalized power spectrogram difference between two tasks for the anterior LFP sites and Fig. 2.15 the same for the posterior LFP sites. Those posterior locations were close to area V6A in the anterior parieto-occipital (POS) sulcus which receives input from extrastriate dorsal visual areas. This area encodes strongly the visual target for reaching and contains mostly visuomotor cells. But anterior locations including area PEa and MIP, which are connected both to V6A and also motor cortex with predominant hand information, contain motor class cells (Battaglia-Mayer et al., 2016). Therefore, we hypothesize that as one goes more anterior task-specificity should get stronger. In other words, we hypothesize to observe stronger difference between the reach and saccade tasks for the LFP sites in the anterior locations compared to the posterior locations.

Interestingly during the fixation hold period we do not see much difference between the tasks for both anterior and posterior location. Our results show that the main difference between two locations happen in the higher beta and gamma bands mainly during the late delay and movement execution. Beta band power decreases in the delay period during reach vs saccade planning much stronger for 
anterior locations than posterior locations (stronger for ipsilateral arm). In addition, contralateral hand selectivity in this band increases more for anterior locations. On the other side, during the movement execution, anterior LFP sites does not show task specificity as much as the posterior LFP sites.

There is a strong increase in gamma power for contralateral reaches compared to saccades for posterior sites (contralateral space selectivity). This also exists during pre-movement and delay periods. But in the anterior MIP, this effect was much weaker. In addition, the contralateral gamma band cue response only exists in posterior MIP but not in anterior locations. Another effect is the significant higher power in lower frequency bands (delta -theta) during delay period in posterior compared to anterior locations which is consistent across conditions and is not hand- or space-selective.

\subsubsection{How different are hand and space representations in MIP versus dorsal pulv- inar?}

We collected data from pulvinar and MIP (but not simultaneously) in two monkeys. Monkeys performed in separate blocks dissociated delay reach (Ddr) and dissociated delay saccade (Dds) tasks. So far we talked about the hand-space encoding in cortical area MIP in the same monkeys. As a general pattern we show that beta band LFP power increases usually during fixation and delay periods and decreased during movement while power in the theta and gamma band increases after cue presentation and movement preparation and execution. This general pattern deviates slightly across monkeys and there are dissimilarities when we compare task-specific responses. Now we ask how similar these representations in MIP are to the representations in the subcortical region dorsal pulvinar. In the following we compare average normalized LFP power in left dorsal pulvinar (monkey $\mathrm{T} n=21$, monkey L $\mathrm{n}=98$ ) with left anterior MIP (monkey $\mathrm{T} \mathrm{n}=44$, monkey $\mathrm{L} \mathrm{n}=82$ ) while the monkeys performed Ddr task as well as Dds task.

First we will show percentage of LFP sites in both MIP and pulvinar which have hand and/or space tuning in different epochs and frequency bands for the dissociated reach task for each monkey separately. We define tuning as a significant difference between normalized LFP power between two conditions across successful trials (two-way ANOVA; see 2.3). Because of the normalized power, this tuning could be in directions of increase or decrease of power.

In addition, we show the difference in power spectrogram at the population level and we also show the difference between two regions (differential profile) in different epoch windows and frequency bands. We averaged normalized power across sites in each frequency band (delta:2-4Hz, theta:4-8Hz, alpha:8-13Hz, low beta: $13-18 \mathrm{~Hz}$, high beta:18-30Hz, low gamma:30-70Hz and high gamma:70$120 \mathrm{~Hz}$ ) and each epoch (fxh (500-0 ms before cue), cue (0-300 ms after cue), del (600-1000 ms after cue), tgo (400-0 ms before movement onset), mvm (0-400 ms after movement onset)) after computing the spectrograms in each task. We did this analysis in each task condition and each space-selectivity and hand-selectivity differential spectrograms (as explained before). We then applied ttest across sites to test for the significance of differences.

\section{Hand and spatial tuning of individual LFP sites}

Figure 2.16 and 2.17 illustrate the percentage of hand and space tuning (calculated by ANOVA on individual LFP sites) in the delayed reach task for the population of LFP sites in left hemisphere 
in monkey $\mathrm{L}$ and monkey $\mathrm{T}$ respectively. In these figures we show the tuning (see 2.3) in the main frequency bands (theta, beta and gamma) in which the highest task-related modulation occurs.

Each figure shows the percentage of tuned LFP sites in area MIP (upper three rows) and also dorsal pulvinar (lower three rows). Each circular pie chart comprises of two concentric circles in which the inner circle depicts the percentage of tuning and the outer circle depicts how much of each tuning has an increased or decreased modulation of power. For example from 82 LFP sites recorded from left MIP in monkey L (Fig. 2.16), 60\% show only ipsilateral hand tuning in their theta band during fixation period. This means that for $60 \%$ of sites absolute normalized power in theta band during fixation was higher for ipsilateral hand than contralateral hand. And in addition, $98 \%$ of those sites (shows as $1 \%$ in the pie chart) had positive (increase) power and 2\% (shows as 59\%) negative (decrease) power.

General effect, seen from the tables, is that we mainly see single tuning (any of IH, CH, IS, CS) but much less double tuning (IH+IS, IH+CS, CH+IS, CH+CS). This means that most of the LFP sites in both areas and also both monkeys show either preference for space (ipsilateral or contralateral) or hand (ipsilateral or contralateral) and to less extend preference for both space and hand.

In most of the cases when there is any tuning, this tuning has either consistent increase or decrease of power across the population of sites. In very few cases a group of tuned sites show subgroups with increased power and subgroups with decreased power and this means that most of the tuned sites have similar pattern of tuning which shows the consistency across population.

Another general point is that, as it has also been shown in other studies such as (Fries et al., 2001; Hwang \& Andersen, 2011), we see also change of tuning direction between low and high frequencies. For example, in monkey $\mathrm{L}$, theta band frequency during cue shows mostly contralateral than ipsilateral space tuning (35\% vs $9 \%$ ) but in gamma band it turns to be mostly ipsilateral than contralateral (28\% vs 9\%). It is worth mentioning that this effect could have two different patterns. One is that a certain type of tuning (e.g. contra-space) in the lower frequencies changes its percentage in favor of opposite type of tuning (e.g. ipsi-space) in the higher frequencies but the mathematical sign (positive vs negative or increase s decrease) of them does not change. Another type would be similar but with a change of mathematical sign. These two patterns have different interpretations because one means the direction of power modulation is the same across frequency bands (e.g. power for contra-space is higher than ipsi-space), but in the other power modulation has different directions across frequencies (e.g. in one frequency contra-space is higher than ipsi-space but in another frequency vice versa). Our data show mostly spatial tuning anticorrelation pattern of first type during cue period and of the second type for the hand tuning during fixation hold. In monkey T, spatial tuning during delay period and hand tuning only in pulvinar shows anticorrelation of second type.

As expected during fixation hold period, in both monkeys, there is almost exclusively hand tuning which is expected because there is no spatial information given yet. In monkey T, beta band power show mostly ipsilateral hand tuning with positive power in both MIP and pulvinar (monkey T dPul $30 \%$ vs 10\%; MIP 66\% vs 9\% ipsilateral vs contralateral respectively). In monkey L, most of MIP sites show contralateral hand tuning with positive power but in pulvinar there is much less hand tuning and for both ipsilateral and contralateral hands (monkey L dPul 11\% vs 18\%; MIP $10 \%$ vs $50 \%$ ipsilateral vs contralateral respectively). 
During delay period, only $35 \%$ of monkey L MIP sites show tuning and there is not a preference for any type of tuning but in the same monkey $62 \%$ of dPul sites show tuning which is mostly hand tuning (more for contralateral hand with decrease of power). In monkey T, 55\% of MIP sites show tuning mostly hand tuning and in favor of ipsilateral hand (increase of power) but $45 \%$ of dPul sites show tuning only for contralateral hand and space (30\% and 15\% respectively; with decrease of power).

Consistent across both monkeys, beta band power during movement in MIP, shows almost exclusively contralateral hand tuning with decrease of power which is also apparent from average power spectrograms (30\% vs $50 \%$ of sites in monkey $\mathrm{L}$ and monkey $\mathrm{T}$ respectively).

In both monkeys there is more spatial tuning than hand tuning after cue presentation in theta, beta and gamma band. In both monkeys, theta band power has more ipsilateral than contralateral space tuning (monkey T 55\% vs $20 \%$ of sites and monkey L $73 \%$ vs $6 \%$ of sites, respectively). Theta band in MIP shows opposite effect. There is more contralateral space tuning with positive power in both monkeys (monkey T $27 \%$ vs $36 \%$ of sites and monkey L $9 \%$ vs 35\% of sites, respectively for ipsi and contra space).

One consistency across monkeys is that ipsilaterally tuned sites show decrease (negative) power and contralaterally tuned sites show increase (positive) power. In both monkeys there are more contralaterally tuned sites with positive power in the theta band for MIP area but more ipsilaterally tuned sites with negative power in dPul. This pattern generally implies that theta and gamma band power is higher for almost all tuned sites for contralateral space than ipsilateral space (contralateral space tuning).

The full results of tuning analysis are reported in the following tables (Table 2.1; Table 2.2; Table 2.3; Table 2.4). Each table contains the percentage of tuned sites (in each of tuning categories) for each frequency band and each epoch, separately for MIP and pulvinar, separately for each monkey. 


\section{LFP sites hand and space tuning in Ddr task (Monkey L)}

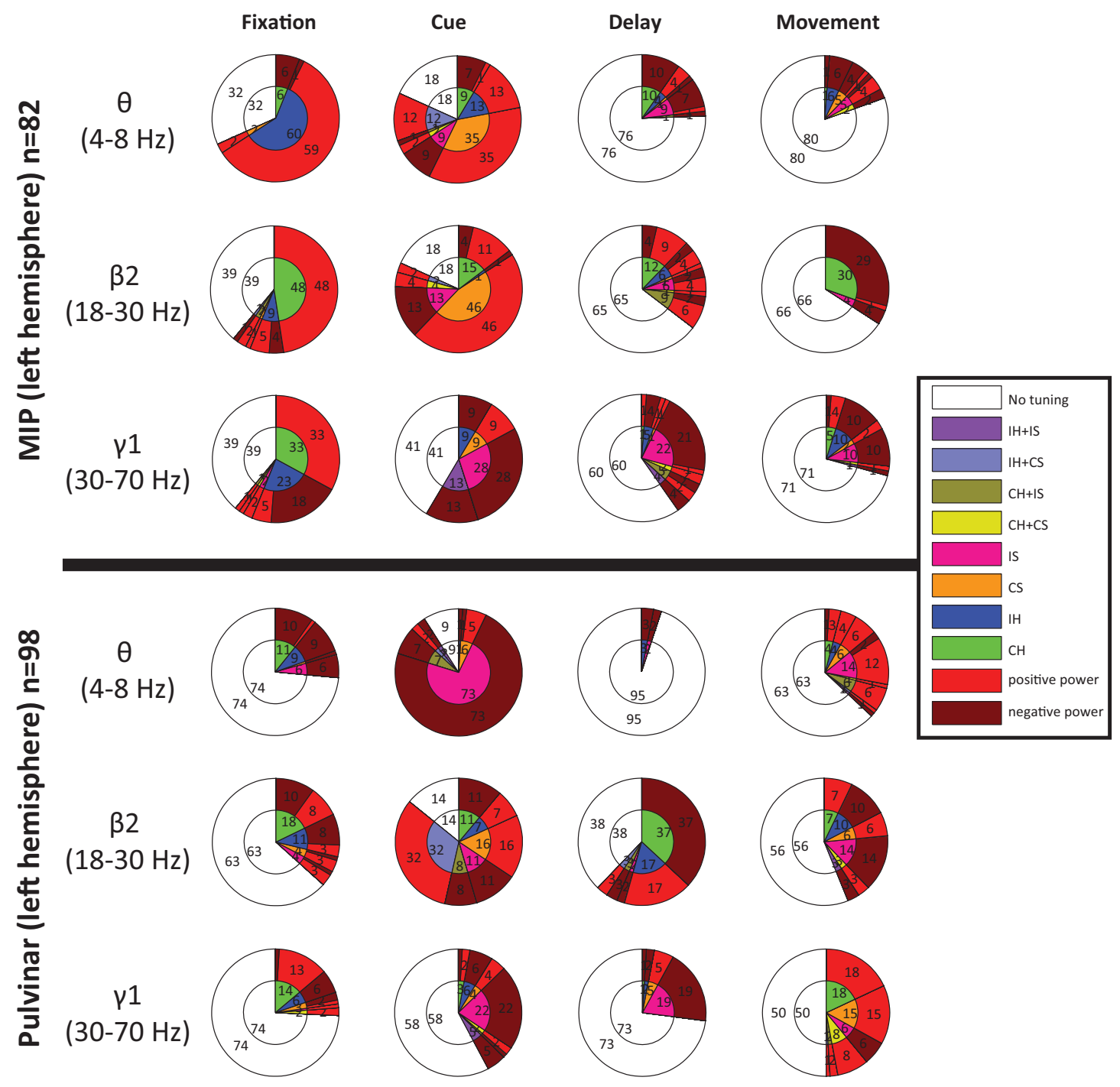

Fig. 2.16 Percentage of hand and space tuning in the population of LFP sites recorded from MIP and pulvinar in left hemisphere of monkey L. Task epochs include fixation (50 ms after fixation hold until $50 \mathrm{~ms}$ before cue onset), cue (50-350 $\mathrm{ms}$ after cue onset), delay (350-50 before go), movement ( $25 \mathrm{~ms}$ after movement onset until $25 \mathrm{~ms}$ before movement offset) . Conditions: IH: ipsilateral hand, CH: contralateral hand, IS: ipsilateral space, CS: contralateral space. Numbers indicate percentage across all recorded LFP sites. 


\section{LFP sites hand and space tuning in Ddr task (Monkey T)}

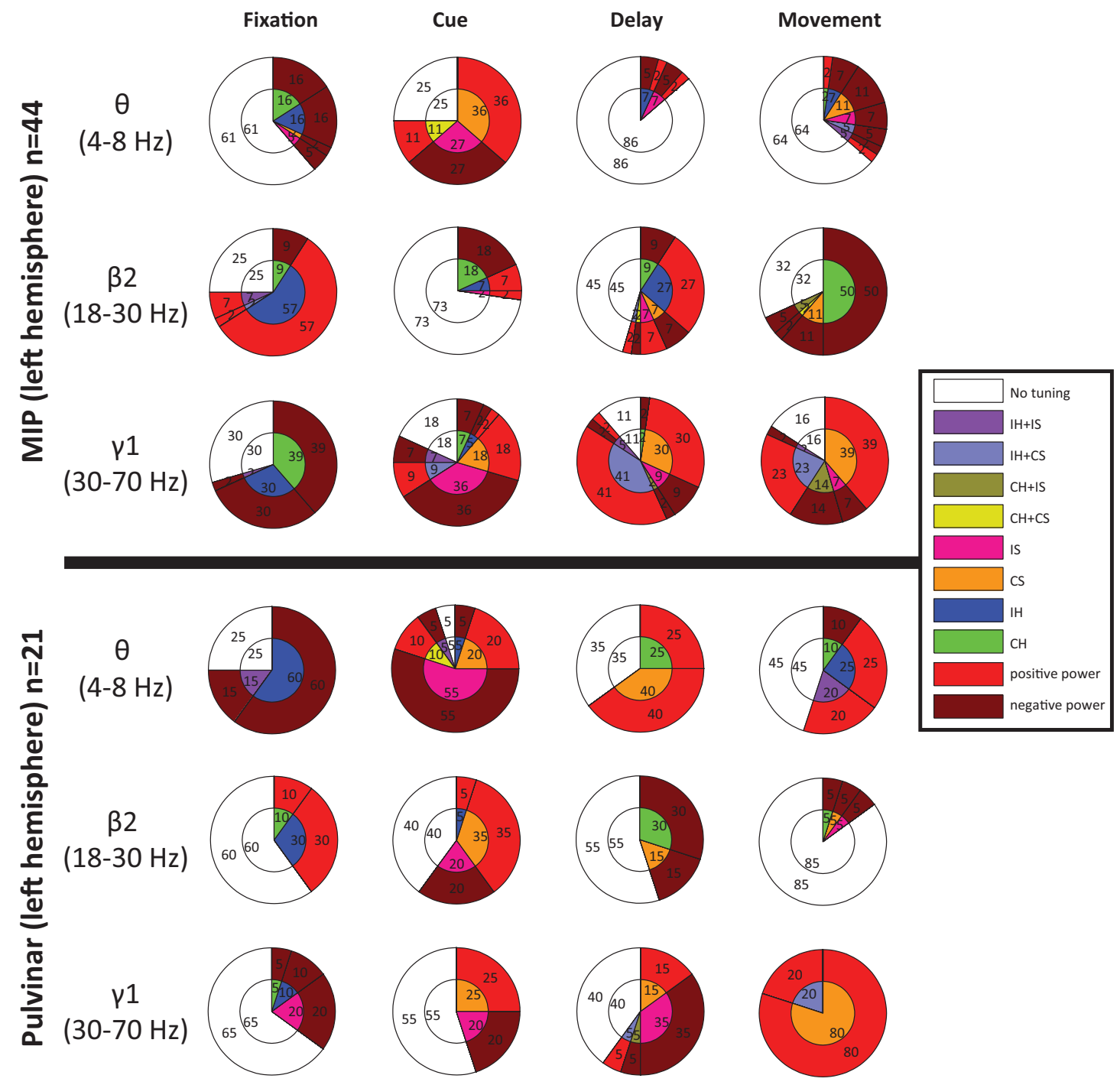

Fig. 2.17 Percentage of hand and space tuning in the population of LFP sites recorded from MIP and pulvinar in left hemisphere of monkey T. Task epochs include fixation (50 ms after fixation hold until $50 \mathrm{~ms}$ before cue onset), cue (50-350 $\mathrm{ms}$ after cue onset), delay (350-50 before go), movement ( $25 \mathrm{~ms}$ after movement onset until $25 \mathrm{~ms}$ before movement offset) . Conditions: IH: ipsilateral hand, CH: contralateral hand, IS: ipsilateral space, CS: contralateral space. Numbers indicate percentage across all recorded LFP sites. 
Table 2.1 Percentage of hand-space tuning in Ddr task (monkey L, MIP, left hemisphere, $n=82$ )

$\begin{array}{lccccccccc} & \text { Not tuned } & \text { IH+IS } & \text { IH+CS } & \text { CH+IS } & \text { CH+CS } & \text { IS } & \text { CS } & \text { IH } & \text { CH } \\ \text { Delta / Fixation } & 62 & 0 & 0 & 0 & 0 & 0 & 1 & 21 & 16 \\ \text { Delta / Cue } & 54 & 2 & 1 & 1 & 1 & 2 & 2 & 17 & 18 \\ \text { Delta / Delay } & 76 & 0 & 0 & 0 & 0 & 1 & 11 & 0 & 12 \\ \text { Delta / Movement } & 50 & 4 & 0 & 0 & 4 & 12 & 13 & 4 & 13 \\ \text { Theta / Fixation } & 32 & 0 & 0 & 0 & 0 & 0 & 2 & 60 & 6 \\ \text { Theta / Cue } & 18 & 0 & 12 & 1 & 2 & 9 & 35 & 13 & 9 \\ \text { Theta / Delay } & 76 & 0 & 0 & 1 & 0 & 9 & 1 & 4 & 10 \\ \text { Theta / Movement } & 80 & 0 & 0 & 0 & 2 & 5 & 5 & 6 & 1 \\ \text { Alpha / Fixation } & 17 & 1 & 0 & 0 & 0 & 2 & 6 & 72 & 1 \\ \text { Alpha / Cue } & 16 & 1 & 11 & 4 & 0 & 20 & 28 & 6 & 15 \\ \text { Alpha / Delay } & 65 & 0 & 0 & 0 & 0 & 17 & 5 & 6 & 7 \\ \text { Alpha / Movement } & 85 & 0 & 0 & 0 & 0 & 0 & 6 & 7 & 1 \\ \text { Beta low / Fixation } & 80 & 0 & 0 & 0 & 0 & 0 & 2 & 6 & 11 \\ \text { Beta low / Cue } & 39 & 1 & 0 & 9 & 0 & 27 & 13 & 6 & 5 \\ \text { Beta low / Delay } & 63 & 0 & 0 & 1 & 0 & 0 & 16 & 9 & 11 \\ \text { Beta low / Movement } & 76 & 0 & 0 & 0 & 0 & 0 & 12 & 2 & 10 \\ \text { Beta high / Fixation } & 39 & 0 & 1 & 2 & 0 & 0 & 1 & 9 & 48 \\ \text { Beta high / Cue } & 18 & 0 & 2 & 0 & 4 & 13 & 46 & 1 & 15 \\ \text { Beta high / Delay } & 65 & 0 & 0 & 9 & 1 & 6 & 1 & 6 & 12 \\ \text { Beta high / Movement } & 66 & 0 & 0 & 0 & 0 & 4 & 0 & 0 & 30 \\ \text { Gamma low / Fixation } & 39 & 0 & 0 & 1 & 1 & 2 & 0 & 23 & 33 \\ \text { Gamma low / Cue } & 41 & 13 & 0 & 0 & 0 & 28 & 9 & 9 & 0 \\ \text { Gamma low / Delay } & 60 & 4 & 0 & 5 & 2 & 22 & 1 & 5 & 1 \\ \text { Gamma low / Movement } & 71 & 1 & 0 & 0 & 1 & 10 & 2 & 10 & 5 \\ \text { Gamma high / Fixation } & 70 & 0 & 0 & 0 & 0 & 2 & 0 & 21 & 7 \\ \text { Gamma high / Cue } & 67 & 1 & 1 & 0 & 0 & 15 & 4 & 9 & 4 \\ \text { Gamma high / Delay } & 90 & 0 & 0 & 1 & 0 & 4 & 2 & 0 & 2 \\ \text { Gamma high / Movement } & 63 & 0 & 0 & 1 & 4 & 1 & 2 & 1 & 27 \\ & & & & & & & & & \end{array}$

Table 2.2 Percentage of hand-space tuning in Ddr task (monkey L, dorsal pulvinar, left hemisphere, $\mathrm{n}=98$ )

$\begin{array}{lccccccccc} & \text { Not tuned } & \text { IH+IS } & \text { IH+CS } & \text { CH+IS } & \text { CH+CS } & \text { IS } & \text { CS } & \text { IH } & \text { CH } \\ \text { Delta / Fixation } & 41 & 1 & 0 & 5 & 0 & 2 & 2 & 11 & 39 \\ \text { Delta / Cue } & 23 & 0 & 0 & 13 & 0 & 57 & 0 & 6 & 2 \\ \text { Delta / Delay } & 80 & 0 & 0 & 0 & 0 & 4 & 4 & 5 & 8 \\ \text { Delta / Movement } & 60 & 0 & 6 & 9 & 0 & 8 & 10 & 6 & 4 \\ \text { Theta / Fixation } & 77 & 0 & 0 & 0 & 0 & 6 & 1 & 9 & 11 \\ \text { Theta / Cue } & 9 & 2 & 2 & 7 & 0 & 73 & 6 & 1 & 0 \\ \text { Theta / Delay } & 96 & 0 & 0 & 0 & 0 & 2 & 0 & 3 & 0 \\ \text { Theta / Movement } & 64 & 1 & 1 & 6 & 1 & 14 & 6 & 4 & 4 \\ \text { Alpha / Fixation } & 82 & 0 & 0 & 1 & 0 & 2 & 2 & 1 & 16 \\ \text { Alpha / Cue } & 5 & 1 & 9 & 3 & 0 & 63 & 21 & 0 & 0 \\ \text { Alpha / Delay } & 89 & 0 & 0 & 0 & 0 & 8 & 1 & 4 & 1 \\ \text { Alpha / Movement } & 66 & 2 & 2 & 1 & 3 & 9 & 10 & 4 & 4 \\ \text { Beta low / Fixation } & 59 & 1 & 1 & 1 & 0 & 0 & 1 & 16 & 24 \\ \text { Beta low / Cue } & 4 & 1 & 15 & 52 & 4 & 14 & 5 & 1 & 6 \\ \text { Beta low / Delay } & 82 & 0 & 0 & 0 & 0 & 4 & 1 & 3 & 10 \\ \text { Beta low / Movement } & 69 & 5 & 0 & 0 & 0 & 6 & 5 & 13 & 4 \\ \text { Beta high / Fixation } & 65 & 0 & 0 & 0 & 0 & 4 & 4 & 11 & 18 \\ \text { Beta high / Cue } & 14 & 0 & 33 & 8 & 0 & 11 & 16 & 7 & 11 \\ \text { Beta high / Delay } & 40 & 0 & 3 & 3 & 0 & 2 & 0 & 18 & 39 \\ \text { Beta high / Movement } & 56 & 3 & 0 & 0 & 3 & 14 & 6 & 10 & 7 \\ \text { Gamma low / Fixation } & 77 & 0 & 0 & 0 & 2 & 1 & 3 & 6 & 14 \\ \text { Gamma low / Cue } & 60 & 5 & 0 & 1 & 2 & 22 & 4 & 6 & 3 \\ \text { Gamma low / Delay } & 74 & 0 & 0 & 0 & 0 & 19 & 5 & 2 & 1 \\ \text { Gamma low / Movement } & 51 & 0 & 1 & 2 & 8 & 6 & 15 & 0 & 18 \\ \text { Gamma high / Fixation } & 88 & 1 & 0 & 0 & 0 & 5 & 1 & 4 & 2 \\ \text { Gamma high / Cue } & 87 & 1 & 0 & 0 & 0 & 6 & 3 & 4 & 2 \\ \text { Gamma high / Delay } & 84 & 1 & 0 & 0 & 0 & 6 & 0 & 5 & 4 \\ \text { Gamma high / Movement } & 72 & 0 & 1 & 3 & 2 & 1 & 14 & 0 & 10\end{array}$


Table 2.3 Percentage of hand-space tuning in Ddr task (monkey T, MIP, left hemisphere, $n=44$ )

$\begin{array}{lccccccccc} & \text { Not tuned } & \text { IH+IS } & \text { IH+CS } & \text { CH+IS } & \text { CH+CS } & \text { IS } & \text { CS } & \text { IH } & \text { CH } \\ \text { Delta / Fixation } & 70 & 7 & 0 & 2 & 0 & 7 & 0 & 11 & 2 \\ \text { Delta / Cue } & 39 & 7 & 9 & 0 & 5 & 18 & 16 & 5 & 2 \\ \text { Delta / Delay } & 59 & 2 & 0 & 0 & 0 & 9 & 2 & 18 & 9 \\ \text { Delta / Movement } & 45 & 2 & 5 & 2 & 11 & 0 & 9 & 18 & 7 \\ \text { Theta / Fixation } & 61 & 0 & 0 & 0 & 0 & 5 & 2 & 16 & 16 \\ \text { Theta / Cue } & 25 & 0 & 0 & 0 & 11 & 27 & 36 & 0 & 0 \\ \text { Theta / Delay } & 86 & 0 & 0 & 0 & 0 & 7 & 0 & 7 & 0 \\ \text { Theta / Movement } & 64 & 5 & 5 & 0 & 0 & 7 & 11 & 7 & 2 \\ \text { Alpha / Fixation } & 57 & 0 & 0 & 0 & 0 & 5 & 0 & 16 & 23 \\ \text { Alpha / Cue } & 27 & 0 & 2 & 7 & 0 & 23 & 41 & 0 & 0 \\ \text { Alpha / Delay } & 70 & 0 & 0 & 2 & 2 & 0 & 2 & 7 & 16 \\ \text { Alpha / Movement } & 75 & 2 & 0 & 0 & 2 & 0 & 18 & 0 & 2 \\ \text { Beta low / Fixation } & 36 & 14 & 0 & 2 & 0 & 0 & 2 & 25 & 20 \\ \text { Beta low / Cue } & 23 & 2 & 5 & 9 & 0 & 41 & 16 & 0 & 5 \\ \text { Beta low / Delay } & 66 & 0 & 2 & 0 & 0 & 2 & 2 & 16 & 11 \\ \text { Beta low / Movement } & 50 & 2 & 0 & 0 & 7 & 0 & 16 & 0 & 25 \\ \text { Beta high / Fixation } & 25 & 7 & 2 & 0 & 0 & 0 & 0 & 57 & 9 \\ \text { Beta high / Cue } & 73 & 0 & 0 & 0 & 0 & 2 & 0 & 7 & 18 \\ \text { Beta high / Delay } & 45 & 0 & 2 & 0 & 2 & 7 & 7 & 27 & 9 \\ \text { Beta high / Movement } & 32 & 0 & 0 & 5 & 2 & 0 & 11 & 0 & 50 \\ \text { Gamma low / Fixation } & 30 & 2 & 0 & 0 & 0 & 0 & 0 & 30 & 39 \\ \text { Gamma low / Cue } & 18 & 7 & 9 & 0 & 0 & 36 & 18 & 5 & 7 \\ \text { Gamma low / Delay } & 11 & 5 & 41 & 2 & 0 & 9 & 30 & 0 & 2 \\ \text { Gamma low / Movement } & 16 & 2 & 23 & 14 & 0 & 7 & 39 & 0 & 0 \\ \text { Gamma high / Fixation } & 48 & 0 & 2 & 0 & 0 & 0 & 0 & 27 & 23 \\ \text { Gamma high / Cue } & 30 & 7 & 5 & 11 & 0 & 18 & 27 & 0 & 2 \\ \text { Gamma high / Delay } & 20 & 0 & 14 & 9 & 9 & 14 & 7 & 18 & 9 \\ \text { Gamma high / Movement } & 41 & 0 & 0 & 0 & 18 & 9 & 25 & 2 & 5\end{array}$

Table 2.4 Percentage of hand-space tuning in Ddr task (monkey T, dorsal pulvinar, left hemisphere, $\mathrm{n}=21$ )

$\begin{array}{lccccccccc} & \text { Not tuned } & \text { IH+IS } & \text { IH+CS } & \text { CH+IS } & \text { CH+CS } & \text { IS } & \text { CS } & \text { IH } & \text { CH } \\ \text { Delta / Fixation } & 45 & 0 & 0 & 0 & 0 & 0 & 0 & 35 & 20 \\ \text { Delta / Cue } & 25 & 20 & 0 & 0 & 0 & 35 & 0 & 15 & 5 \\ \text { Delta / Delay } & 60 & 0 & 0 & 0 & 0 & 0 & 40 & 0 & 0 \\ \text { Delta / Movement } & 45 & 0 & 0 & 0 & 5 & 25 & 5 & 0 & 20 \\ \text { Theta / Fixation } & 25 & 15 & 0 & 0 & 0 & 0 & 0 & 60 & 0 \\ \text { Theta / Cue } & 5 & 5 & 0 & 0 & 10 & 55 & 20 & 5 & 0 \\ \text { Theta / Delay } & 35 & 0 & 0 & 0 & 0 & 0 & 40 & 0 & 25 \\ \text { Theta / Movement } & 45 & 20 & 0 & 0 & 0 & 0 & 0 & 25 & 10 \\ \text { Alpha / Fixation } & 85 & 0 & 5 & 0 & 0 & 0 & 0 & 5 & 5 \\ \text { Alpha / Cue } & 0 & 0 & 0 & 0 & 5 & 5 & 90 & 0 & 0 \\ \text { Alpha / Delay } & 80 & 0 & 0 & 0 & 0 & 0 & 5 & 5 & 10 \\ \text { Alpha / Movement } & 70 & 5 & 0 & 0 & 0 & 5 & 10 & 5 & 5 \\ \text { Beta low / Fixation } & 85 & 0 & 0 & 0 & 0 & 0 & 0 & 0 & 15 \\ \text { Beta low / Cue } & 0 & 0 & 0 & 0 & 5 & 0 & 95 & 0 & 0 \\ \text { Beta low / Delay } & 65 & 0 & 0 & 0 & 0 & 0 & 0 & 5 & 30 \\ \text { Beta low / Movement } & 85 & 0 & 0 & 0 & 0 & 5 & 0 & 10 & 0 \\ \text { Beta high / Fixation } & 60 & 0 & 0 & 0 & 0 & 0 & 0 & 30 & 10 \\ \text { Beta high / Cue } & 40 & 0 & 0 & 0 & 0 & 20 & 35 & 5 & 0 \\ \text { Beta high / Delay } & 55 & 0 & 0 & 0 & 0 & 0 & 15 & 0 & 30 \\ \text { Beta high / Movement } & 85 & 0 & 0 & 0 & 0 & 5 & 5 & 0 & 5 \\ \text { Gamma low / Fixation } & 65 & 0 & 0 & 0 & 0 & 20 & 0 & 10 & 5 \\ \text { Gamma low / Cue } & 55 & 0 & 0 & 0 & 0 & 20 & 25 & 0 & 0 \\ \text { Gamma low / Delay } & 40 & 0 & 5 & 5 & 0 & 35 & 15 & 0 & 0 \\ \text { Gamma low / Movement } & 0 & 0 & 20 & 0 & 0 & 0 & 80 & 0 & 0 \\ \text { Gamma high / Fixation } & 75 & 0 & 0 & 0 & 0 & 0 & 5 & 20 & 0 \\ \text { Gamma high / Cue } & 70 & 0 & 0 & 0 & 0 & 20 & 5 & 0 & 5 \\ \text { Gamma high / Delay } & 70 & 0 & 0 & 0 & 0 & 0 & 15 & 0 & 15 \\ \text { Gamma high / Movement } & 65 & 0 & 5 & 0 & 5 & 0 & 15 & 5 & 5\end{array}$


So far we characterized the hand and spatial preference of individual LFP sites while the monkey performed the dissociated reach task (Ddr) in both MIP and dPul areas. Next we asked how different the population of LFP sites in those areas encode hand and space variables. By the previous tuning analysis we just have information about the preference and the direction (positive or negative) of the tuning but that analysis does not give us information about the depth or strength of the tuning. We pooled together the power spectrogram of individual LFP sites (averaged across trials) in each condition separately and compared in population representation between MIP and dPul.

\section{MIP vs dPul population power spectrum in Ddr task}

In Figure 2.18 we first show how population of LFP sites in dorsal pulvinar in left hemisphere encode hand and space variables in Ddr and Dds tasks. In both monkeys there is a significant contralateral space cue response in the range of theta to beta power. Moreover and consistent across two monkeys, beta band power (which is stronger in low beta in monkey $\mathrm{L}$ and high beta in monkey $\mathrm{T}$ ) has an increase during fixation hold (before cue presentation) and spatial target hold. But beta power during delay period in monkey $\mathrm{L}$ which is dominantly stronger for contralateral hand, has different dynamics in the other monkey. In monkey $\mathrm{T}$, beta power tends to decrease during delay period in all conditions but slightly stronger for contralateral hand. According to this and for both monkeys, delay period beta band power is lowest for the contralateral hand. This effect was expected from the tuning analysis.

Interestingly consistent across both monkeys, beta band power is lower for contralateral hand than ipsilateral hand in all holding states. In addition, in both monkeys, gamma as well as theta band power modulate around the movement execution. In monkey L, theta band increases during movement in all conditions but stronger for crossed hand-space conditions (IHCS, CHIS) but in monkey T it only increases for the uncrossed hand-space conditions (IHIS, CHCS). In addition, delta-theta band power in monkey L decreases mostly for the ipsilateral space during cue and delay periods, while in monkey $\mathrm{T}$ it decreases during holding periods and increases during delay period (stronger for contralateral hand). The gamma band shows slight increase after cue and also stronger increase during and before movement which is higher for contralateral space in both monkeys.

One explanation why we see delta-theta band increase as well as slight increase in gamma in monkey $\mathrm{T}$ during late delay period in all conditions would be due to his performance and movement preparation and execution. This monkey in general had a lower performance compared to monkey $\mathrm{L}$ (65\% versus $85 \%$, respectively). Monkey T made many error during holding time period which means for him it was difficult to maintain his eye and hand fixation for several hundred milliseconds. Most probably he started to prepare movement (or even do some small hand movement) while maintaining fixation in the successful trials.

In the dissociated saccade task, both monkeys show contralateral spatial cue response in the theta-alpha range. While monkey $L$ has slight increase of beta activity during fixation hold, in monkey $\mathrm{T}$ it is stronger (especially for the contralateral hand). On the other hand, beta activity during delay in monkey $\mathrm{L}$ increases more than monkey $\mathrm{T}$ and this increase in both monkeys are stronger for ipsilateral hand. In addition, while in monkey L gamma band activity is weak and just slightly increases during saccade, in monkey $\mathrm{T}$ gamma band is slightly stronger and contralaterally tuned for space. 
Averaged normalized power spectrogram across sites in left pulvinar (Ddr task)

a) monkey $L(n=98)$

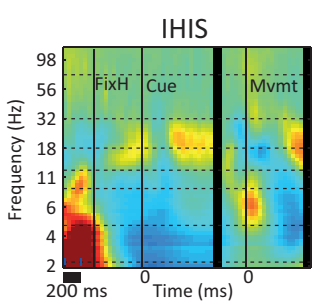

CHIS

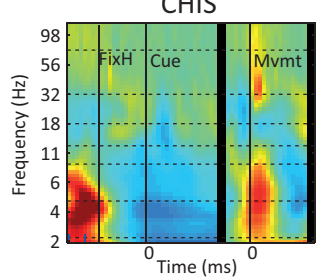

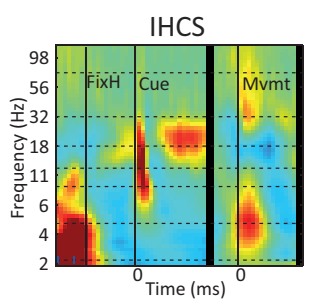

CHCS

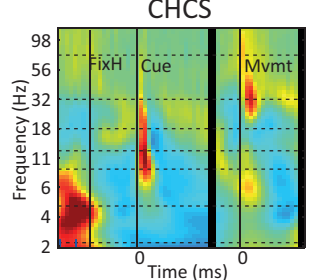

b) monkey $T(n=21)$
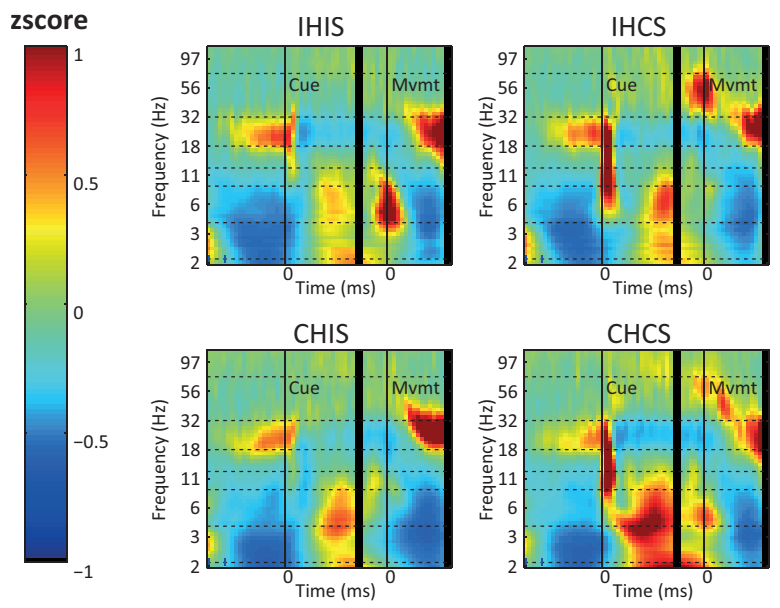

\section{Averaged normalized power spectrogram across sites in left pulvinar (Dds task)}

c) monkey $L(n=93)$
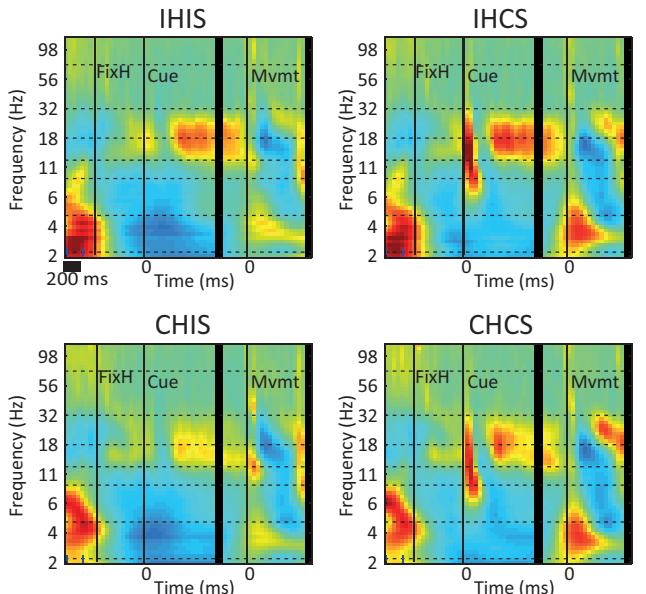

d) monkey $T(n=19)$
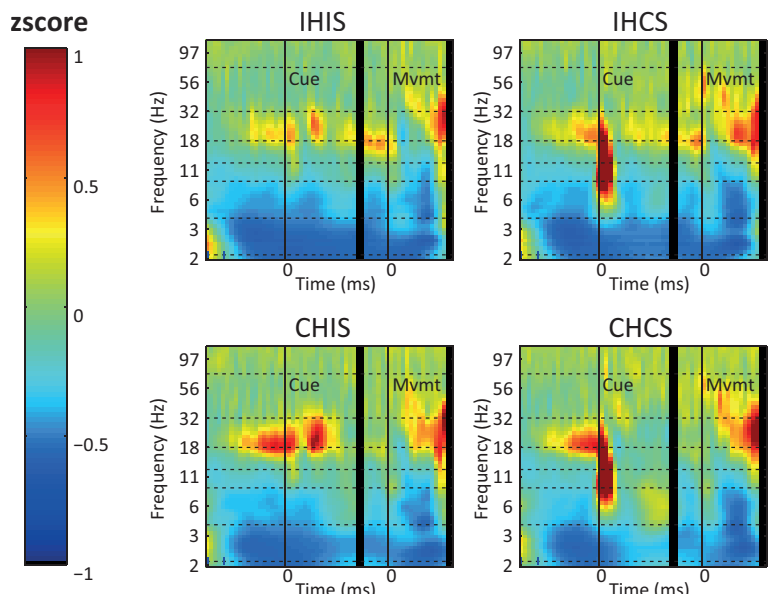

Fig. 2.18 Averaged normalized power spectrogram across sites recorded from dorsal pulvinar in left hemisphere while monkeys performed dissociated delay reach task (a) in monkey L and (b) in monkey T, and dissociated delay saccade task (c)monkey L and (d) monkey T. Spectrograms are aligned to cue and movement onset. Note that in monkey L fixation hold period was shorter than monkey T. I: ipsilateral, C: contralateral, H: hand, S: space. Ipsi and contra relative to the recorded hemisphere. 
Now that we have an overview of dorsal pulvinar power distribution for reach and saccade behavior, we ask how different are these responses as compared to the cortical area MIP. Figure 2.19 illustrates the difference between average normalized LFP power across sites in area MIP and dPul in monkey $\mathrm{T}$ (a) and monkey L (b) while the monkeys performed dissociated reach (Ddr) task.

In both monkeys, but stronger in monkey L, we observe a significant higher power in below beta frequencies during the initial fixation period in MIP compared to pulvinar. Delta-theta-alpha cue response is higher in MIP than in dPul for both monkeys but stronger in monkey L. We also see a slight but significant higher power in gamma for contralateral space after cue in monkey $\mathrm{T}$ which is much weaker in monkey L.

In monkey T, beta band power during delay and go period is higher in MIP than in dPul, stronger for ipsilateral arm. But in monkey L, we see an increase for contralateral arm but slight decrease for ipsilateral arm, which leads to higher contralateral hand selectivity in MIP than dPul. In addition, in monkey L opposite to monkey $\mathrm{T}$, there is higher power in the lower frequency bands (delta-theta-alpha) in this period in MIP relative to $\mathrm{dPul}$.

In the pre-movement (go) period, similarly in both monkeys, theta and alpha power is significantly higher in pulvinar than MIP. Although this is a general similar pattern across two monkeys but regarding the hand and space selectivity (contralaterality) they show opposite effects. Moreover, while monkey $\mathrm{L}$ generally shows lower power in MIP than dPul during the movement execution, this effect is different in monkey T. In this monkey we see slightly higher power in MIP than dPul during movement.

In monkey $\mathrm{T}$, hand contralaterality maps tend to contain bluish colors which means contralateral hand tuning is lower in MIP than in dPul. Interestingly gamma band power has a stronger contralateral space selectivity in MIP than in pulvinar. In monkey L, hand contralaterality is higher in MIP than dPul mostly for the beta band power and less in gamma band. In addition, cue response in alpha-beta1 band power shows lower contralateral space tuning in MIP than in dPul, in both monkeys.

\section{MIP vs dorsal pulvinar population power spectrum in Dds task}

Figure 2.20 illustrates the difference between average normalized LFP power across sites in area MIP and $\mathrm{dPul}$ in monkey $\mathrm{T}$ (a) and monkey L (b) while the monkeys performed dissociated saccade (Dds) task.

Generally, there is more difference between MIP and dPul representations in the saccade task compared to the reach task (more significant different bins). As our data depicts, consistent across monkeys, LFP power in lower bands (delta-theta and partially alpha) are generally higher in MIP compared to dPul for saccades. This effect was present only during fixation and cue in reach task. The beta band power during fixation hold show different patterns across two monkeys. In monkey L we see higher power in MIP than dPul for all conditions but in monkey $\mathrm{T}$ higher power in MIP for ipsilateral arm but opposite for the contralateral arm. This means for our population of LFP sites, during fixation period, dPul has a different hand tuning than MIP and in a different direction across monkeys. This effect was seen also in the reach task. 
Epoch and frq-band specific difference of zscored power in between areas a) MIP(n:44)-PUL(n:21), Ddr task instructed trials, monkey T left hemisphere
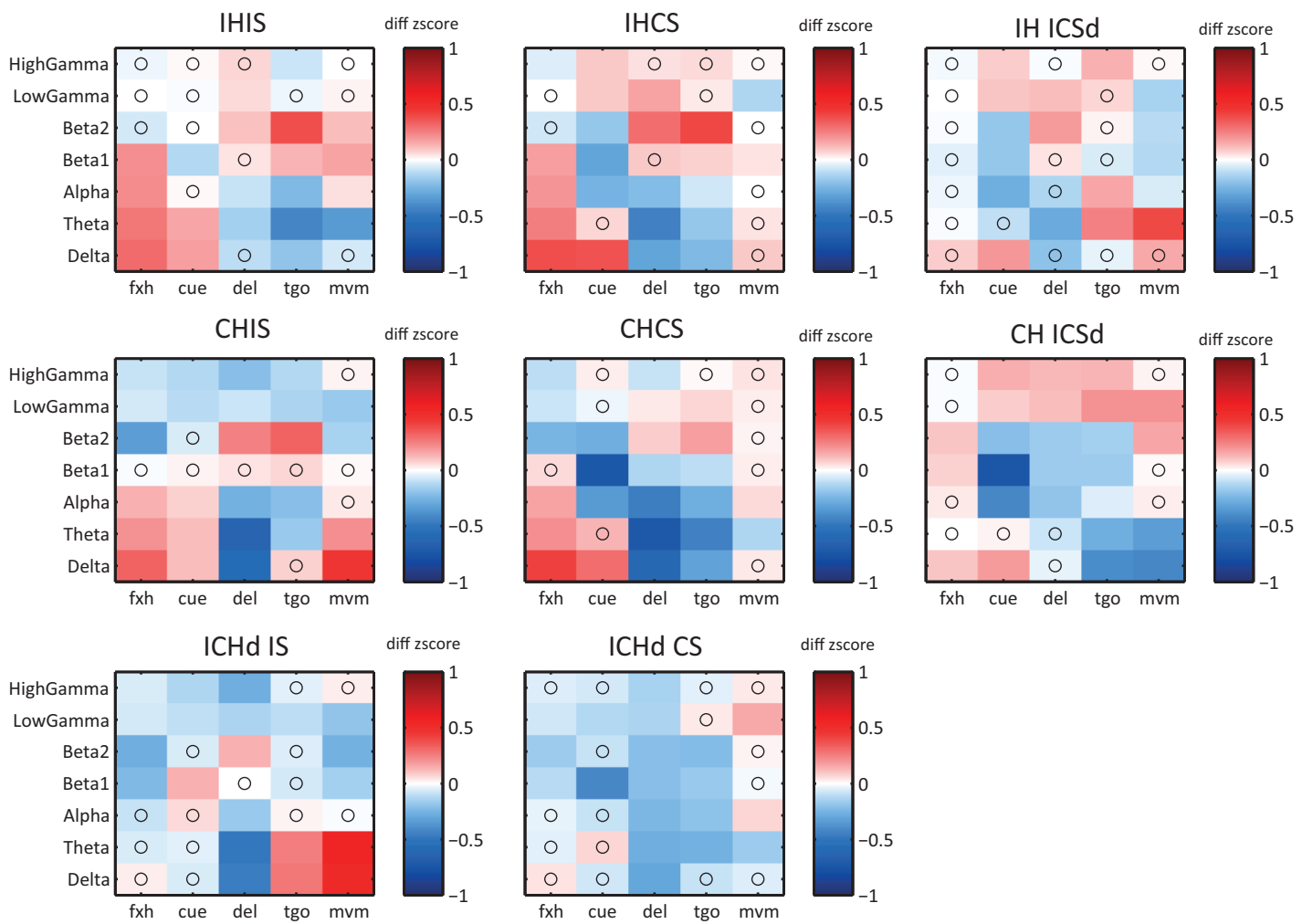

b) $M I P(n: 82)-P U L(n: 98)$, Ddr task instructed trials, monkey L left hemisphere
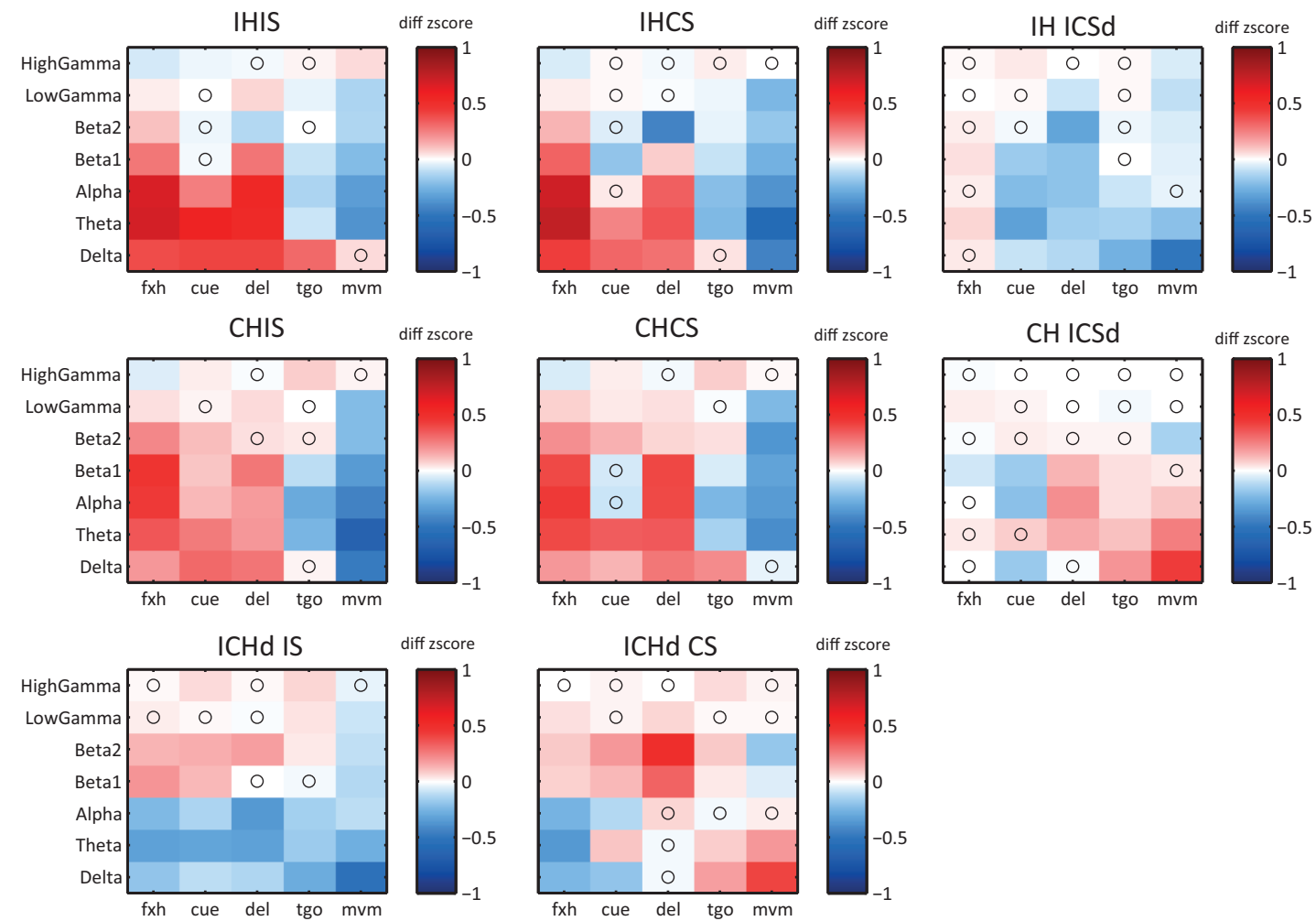

Fig. 2.19 Difference between normalized power in MIP and dPul in each frequency band and each epoch (fxh (500-0 ms before cue), cue (0-300 ms after cue), del (600-1000 ms after cue), tgo (400-0 ms before movement onset), mvm (0-400 ms after movement onset)) in monkey $\mathrm{T}$ (a) and monkey L (b) while they performed dissociated delay reach task (Ddr). Color in each bin shows the actual difference. Significance computed by non-paired ttest across sites. Empty circles show non-significant bins. Condition abbreviations: IH (ipsi hand), CH (contra hand), IS (ipsi space), CS (contra space), ICSd (contra-space minus ipsi-space), ICSd(contra-hand minus ipsi-hand) 
Epoch and frq-band specific difference of zscored power in between areas a) MIP(n:23)-PUL(n:19), Dds task instructed trials, monkey T left hemisphere

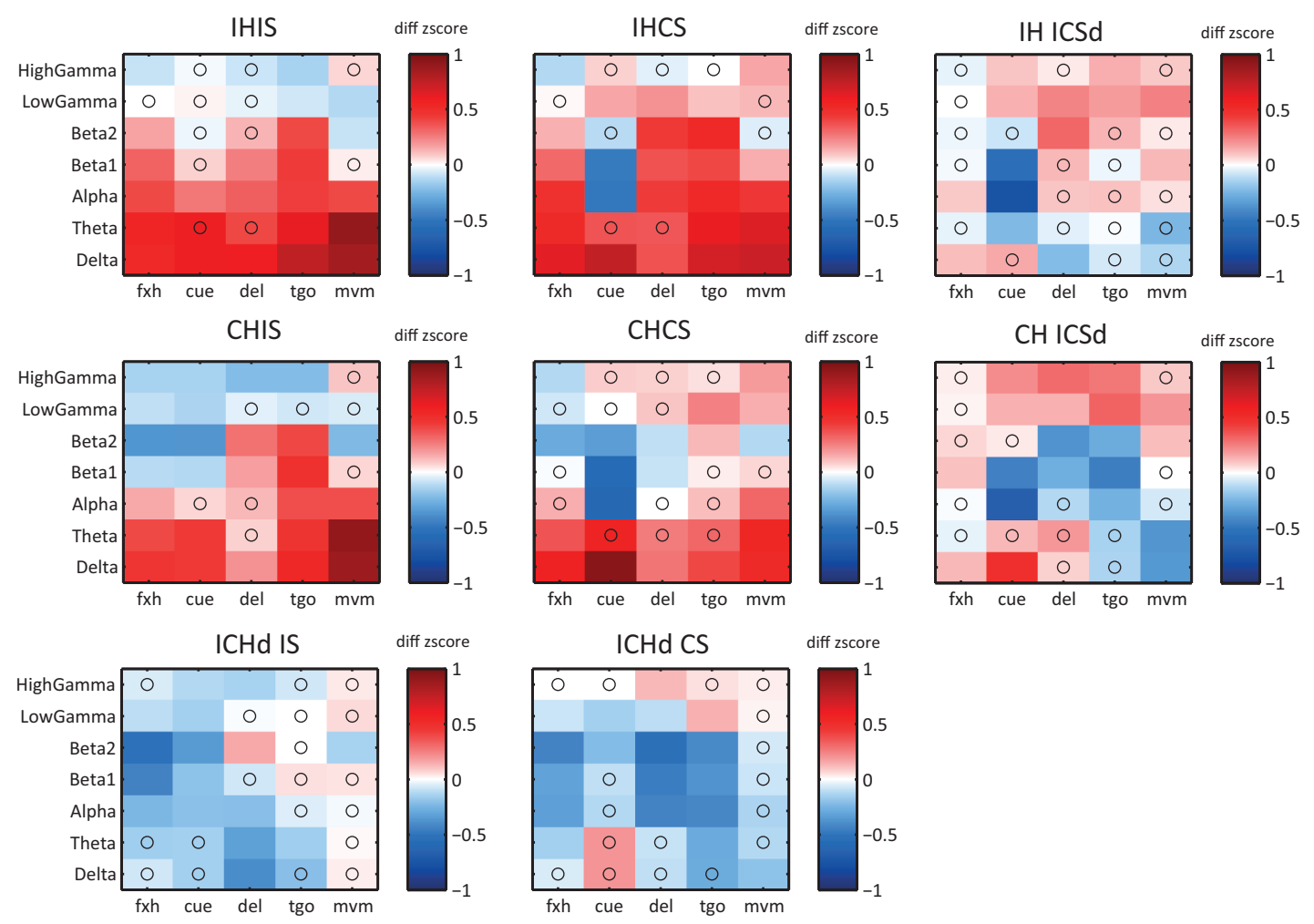

b) MIP(n:36)-PUL(n:93), Dds task instructed trials, monkey L left hemisphere

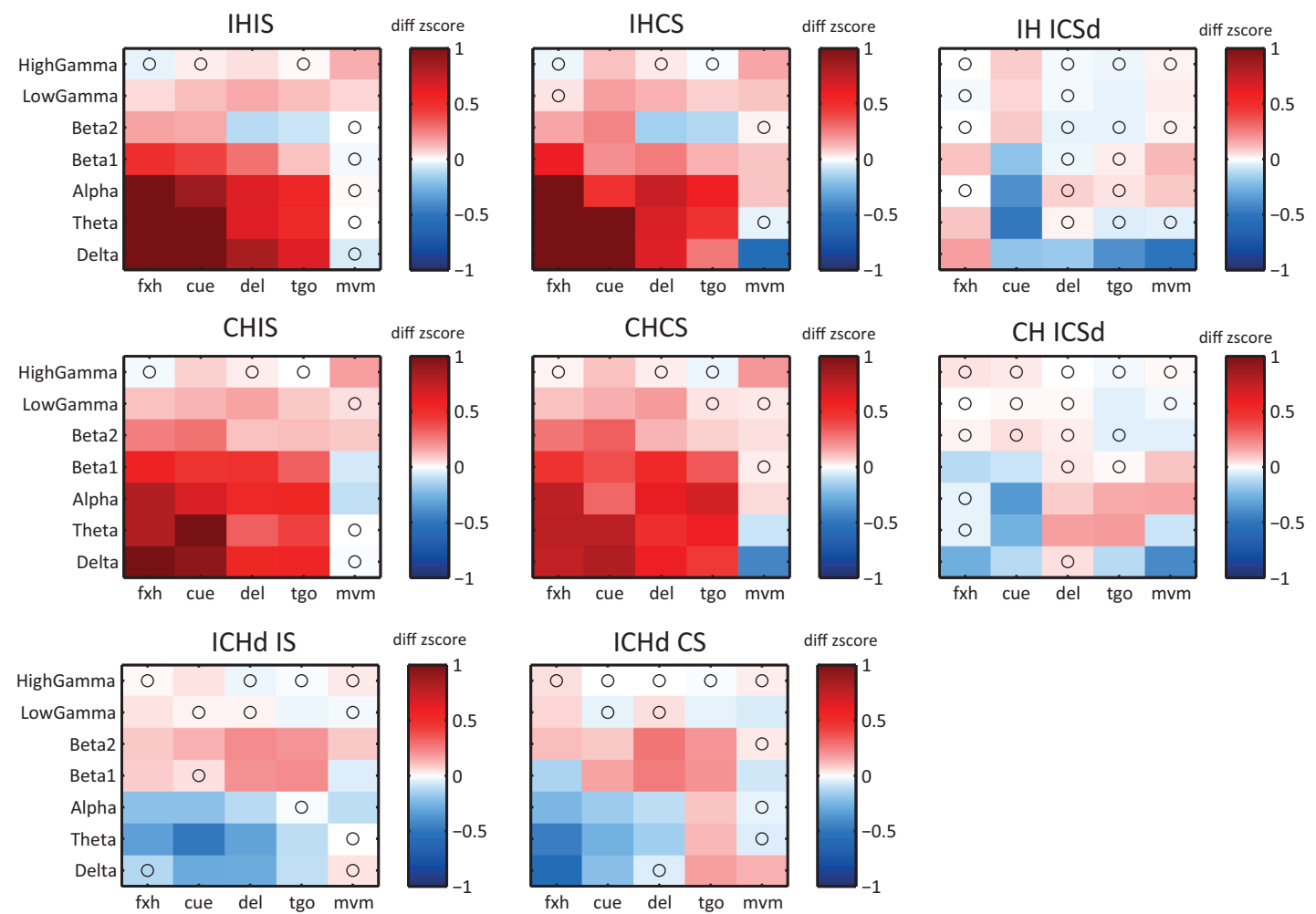

Fig. 2.20 Difference between average normalized LFP power in MIP and pulvinar for each frequency band and each epoch in monkey T (a) and monkey (L) while they performed dissociated delay saccade task (Dds). Similar to Fig. 2.19 
Moreover, there is a significant decrease in power in MIP relative to dPul after cue presentation in the alpha-beta range only for the contralateral space in monkey $\mathrm{T}$. In other words, the contralateral cue response in dPul is stronger than in MIP in that frequency range. But in monkey L, there is a general increase in pulvinar for the same comparison. Consistent across monkeys, this frequency band has a lower contralateral space selectivity for cue in MIP than in dPul.

Looking at the hand-selectivity map, for monkey $\mathrm{T}$, the overall bluish color implies that the hand-contralaterality is stronger in $\mathrm{dPul}$ than MIP on the population average level. Similar effect was present in the Ddr task. While in monkey T, delay period beta band hand contralaterality was lower in MIP only for the contralateral space, in monkey L, hand contralaterality was generally higher in MIP than dPul (consistent with Ddr task).

In monkey T, gamma band space contralaterality is higher in MIP than in pulvinar (consistent with Ddr task). In monkey L, hand contralaterality in the beta band range is generally higher for MIP than pulvinar but it is higher in pulvinar in lower frequency bands.

\subsection{Discussion}

In this comparative study we characterized for the first time the similarity and differences of local field potential power distributions in dorsal aspect of subcortical thalamic pulvinar versus parietal cortical area MIP for dissociated reach and saccade tasks. Although several studies investigated the oscillatory activity in frontoparietal reach-related areas in terms of spatial representations (Dean et al., 2012; Hwang \& Andersen, 2011; Pesaran et al., 2008; Scherberger et al., 2005; Wong et al., 2016) but up to our knowledge this is the first study in which hand-, eye- and space-specific responses are compared. In addition, to the best of our knowledge no previous study investigated the encoding of reach and saccade in dorsal pulvinar local field potentials. For that we trained two rhesus monkeys to perform three different tasks: dissociates delayed saccade (Dds), dissociated delayed foveal reach (Ddr) and extra-foveal reach (Ddf). In each of those tasks monkeys were cued to use effectors based on the cue color (red: eye, blue: left hand, green: right hand).

\subsubsection{General differences in power spectrum across monkeys}

Comparing the power spectrum across regions revealed that LFP power in $\mathrm{dPul}$ is generally lower than in cortex in both monkeys. The cytoarchitecture of cells in cortex is more structured than in pulvinar which cause stronger synchronous dipoles in cortical area and consequently larger LFPs (Buzsáki et al., 2012). But apart from that, both MIP and dPul raw power spectrums revealed that in both regions beta power plays a major role in reach and saccade movement planning, though the pattern has dissimilarities across monkeys. Beta band power is sharper and peak at lower beta frequencies in monkey $\mathrm{L}$ but is broader and peak at higher beta frequencies in monkey $\mathrm{T}$. This might be due to the age and size differences, as it has been shown that beta band properties in motor cortex changes by aging. For example motor cortex beta band power in older human tends to be higher and have peak in lower frequencies (Rossiter et al., 2014). Another major frequency component in both regions is the gamma band which is mostly higher around the movement time. On the other hand, lower frequency bands (delta-theta) power increase during movement in dPul which is not the same in MIP. 


\subsubsection{Dorsal pulvinar and MIP encode eye-, hand- and space similarly}

One expected pattern which is confirmed in our data is that beta band power in the fixation hold period did not change much across tasks. This is expected because during this epoch all tasks are similar although tasks were performed in blocks. Relative to the fixation hold, beta band decreases during delay, though stronger in movement, while gamma power increases (stronger in monkey T) (Dean et al., 2012). In addition, beta attenuation during movement which has been shown before in motor cortical areas during active and imaginary movements (Fry et al., 2016; Jensen et al., 2005; Jurkiewicz et al., 2006; Pfurtscheller et al., 2005), was much less in monkey T than monkey L. One potential reason could be that monkey $\mathrm{T}$ was impatient to wait for the go cue during delay periods and he was in movement prepaation mode which matched to his behavioral data (many delay period aborts and generally lower performance relative to monkey L). But Similar across monkeys and in both MIP and $\mathrm{dPul}$, power of beta band during fixation hold is higher for reaches than saccades but during delay and pre-movement is generally lower for reaches than saccades, although the strength of the contralateral selectivity is different across monkeys and regions. This means the level of beta attenuation during delay compared to fixation hold is stronger in reaches than saccades which is in line with previous studies in parietal cortex (Dean et al., 2012; Hagan et al., 2012; Hwang \& Andersen, 2011) and is a sign for reach-specificity of MIP as well as pulvinar.

In both monkeys and mostly in $\mathrm{dPul}$, theta band power is higher in reaches than in saccades during pre- and peri-movement periods. It has been shown that theta band oscillations in thalamus is important for sustained attention (Yu et al., 2018; Zhou et al., 2016) but its role in movement preparation is not known. Our results suggest that it may be also functional in an effector-specific manner in higher order thalamus maybe through corticothalamic cross-frequency coupling of oscillatory activity for example theta-gamma phase-amplitude coupling (Aru et al., 2015).

In both MIP and dPul and also partially similar across monkeys, gamma band power is slightly higher in reaches than saccades although the direction of tuning is not consistent across regions. But consistent across monkeys dPul gamma band has higher spatial contralateral preference in reaches than saccades, which implies that dPul gamma band has stronger spatial tuning in reaches than saccades.

On the other hand, in general LFP spectral pattern in extrafoveal reaches (Ddr task) is much more similar to foveal reach (Ddf task: reach accompanied with saccade) than dissociated saccade (Dds task) in both monkeys. In other words, when comparing extrafoveal with foveal reach versus with saccades, general difference pattern stays similar but the difference is stronger between reach and saccade (in both MIP and dPul; dPul data not shown). Consistent across monkeys and in both dPul and MIP, beta band power in Ddr is lower than in Ddf (dPul data is not shown). Since in foveal and extra-foveal reaches different set of eye-hand coordination is required for performing a proper reach movement, a difference in neuronal oscillations suggests that they might be involved in eye-hand coordination beside a pure reach goal encoding (Dean et al., 2012; Hwang et al., 2012, 2014; Jackson et al., 2005; Yttri et al., 2014). Interestingly our data shows that dPul might also be important for those transformations not weaker than MIP, a role that is still not understood and needs more investigation.

Although gamma frequency activity during delay and pre-movement is stronger in MIP but it is stronger during movement in $\mathrm{dPul}$. In both regions gamma band power during movement to contralateral space is higher in Ddr than in Ddf and Dds (contralateral spatial preference). This is 
partially consistent across monkeys. The controversial point is that in monkey $\mathrm{T}$ this effect is stronger in MIP than in dPul but in monkey L the effect is much weaker or even reverse in MIP.

\subsubsection{Frequency specific tuning in MIP and dPul}

We also characterized space or hand preference of individual LFP sites and asked whether at the population level percentage of LFP sites with a certain tuning differs depending on the epoch and frequency band and whether this is different across MIP and dPul.

As a general effect and similar to previous reports (Fries et al., 2001; Hwang \& Andersen, 2009, 2011), we observed that direction of tuning changes from low to high frequencies. Since this effect does not exist in all epochs, it would not be an intrinsic attribute of LFP oscillations, rather it might origin from other sources such as task related cross frequency communications. In one study, (Hwang \& Andersen, 2011), for example, observed that spatial tuning of PRR LFPs in $10-20 \mathrm{~Hz}$ and $20-30 \mathrm{~Hz}$ differ not only within a task but also across tasks (memory vs visually-guided reach). In addition, they observed gamma band to be tuned mostly contralaterally (similar to (Hwang \& Andersen, 2012)) but low and high beta range power to be less tuned and in both directions (consistent with our results). In another study, (Hwang \& Andersen, 2012) showed that beta band LFP power in PRR has preference directed upward on the mid-line (not ipsi- or contralateral).

According to our tuning analysis, there is no clear pattern across frequencies and monkeys about hand and space tuning. Although there are a few similarities between MIP and dPul, it seems that both regions encode hand and space but at a different level and in a miscellaneous manner.

One similarity is that during fixation both regions mostly show hand tuning (as expected because there is not spatial information available) but the pattern is different between monkeys as it was also observed in the averaged spectrograms. Only less than half of LFP sites showed beta band power tuning during delay and movement in both monkeys. And those care more about hand than space and it is lower for contralateral hand and/or higher for ipsilateral hand.

On the other hand, both MIP and dPul represent stronger contralateral spatial cues than ipsilateral which is consistent across monkeys. Theta band and to less degree gamma band oscillations both show mostly spatial tuning in the direction of contralateral targets. Interestingly in both regions and again consistent across monkeys, more percentage of LFP sites showed spatial tuning than hand tuning in the range of theta and gamma bands after cue presentation and also later in trial during delay and movement preparation (see (Hwang \& Andersen, 2011, 2012; Scherberger et al., 2005)). Another interesting finding is that higher percentage of LFP sites has theta band contralateral spatial tuning in dPul compared to MIP but gamma band shows the opposite pattern. This implies that gamma band has a higher function role in spatial encoding in cortex than lower frequencies while the frequency-dependent dominance is opposite in higher order thalamus.

Given the fact that the percentage of tuning (for both space and hand) changes during trial (dynamic with time or temporal changes in tuning), this can imply two changes in the system: either individual LFP sites change their tuning by time as the movement preparation and execution progress, or this changes are due to the reorganization in the population. This means that while group of sites encode movement preparation other group are more involved in the movement itself. To test this, we need to do the same analysis using sliding window stepwise through the whole trial to understand the dynamics of hand and space tuning within each site. In a similar approach (Lehmann \& Scherberger, 
2015) showed that LFP spatial tuning in area AIP is highest in the beta band oscillations and less in the gamma band and it starts after cue and remains through the trial. They show ipsi and bottom-ipsi ward spatial tuning.

\subsubsection{Anterior and posterior MIP contribute differently to visuomotor behavior}

Although different locations within superior parietal lobe (SPL) show reach related responses but the properties and the strength of effector and spatial preferences seems to be different (Battaglia-Mayer et al., 2016). For example while area V6A located at rostral bank of POS does early stages of visual transformation for reaches and grasping, area 5 and MIP located more anterior long the IPS are responsive for visually-guided arm movement (Caminiti et al., 2015). Moreover lesions to those areas cause different visuomotor deficits (Caminiti et al., 2010). Only in monkey T, we recorded from locations in the posterior part of MIP (closer to V6A) and also more anterior parts of MIP to characterize the differences in visuo-motor responses between two areas. Since V6A receive large visual inputs from eye-movement related area such as LIP and has less direct connections to motor cortex, we expected that compared to MIP locations close to V6A dissociate less between reach and saccade and this might emerge in less beta band tuning and higher gamma band tuning in that area.

Our results show that the main difference between two locations happen in the beta and gamma bands mainly during the late delay and movement execution. Lower beta band power in the delay period during reach versus saccade planning is much stronger for anterior locations than posterior locations (stronger for ipsilateral arm). In addition, contralateral hand selectivity in this band increases more for anterior locations.

On the other hand, during the movement execution, anterior LFP sites do not show task specificity as much as the posterior LFP sites in the gamma band. There is a strong increase in gamma power for contralateral reaches compared to contralateral saccades for posterior sites (contralateral space selectivity). This also exists during pre-movement and delay periods. But in the anterior MIP, this effect was much weaker. In addition, the contralateral gamma band cue response (in reach versus saccade) only exists in posterior MIP but not in anterior locations. Another effect is the significant higher power in lower frequency bands (delta-theta) during delay period in posterior compared to anterior locations which is consistent across conditions and is not hand- or space-selective. This result confirms our predictions and suggests that posterior locations along the IPS have more visuomotor than motoric functions. These results confirm that posterior locations of MIP are more involved in spatial than limb encoding with stronger contralateral preference. Also it seems that these two regions have less similarity in saccade encoding than reach encoding.

\subsubsection{Future directions and summary}

We have shown the differences between regions and tasks at the population level (as average LFP power spectrum) and we showed as scatter plots whether the effects on the individual LFP sites was consistent across population. To be able to quantify the task-specific or region-specific modulations, we attempt to measure for each LFP site the differences (modulation index) in each frequency band and epoch and plot the distribution of indices for the population. Shape and statistics of the distribution will give us a better look for consistency across population. 
Another aspect of data is the LFP-LFP pairwise phase consistency to measure the level of synchronization in each of those areas for different tasks. It is expected that the level of local synchronization in area MIP would be higher in dissociated reach versus saccade task but the question is whether level of synchrony is different when monkey is allowed to look and reach (foveal reach). The next question would be whether local field synchronization is lower in general in dPul than MIP and how task-dependent is that.

There are several other analyses which are still under investigation: For example, is there any correlation between behavioral properties such as movement time and reaction time and LFP power in different frequency bands during delay, pre- and peri-movement periods? Previously, (Dean et al., 2012) have shown that LFP power in beta and gamma bands in area LIP and PRR correlate with reach and saccade reaction times. Is the strength of correlations stronger in reach than saccade task independent of region (MIP vs dPul) or the correlation is stronger in MIP? The other question is whether the correlations in foveal reach is linear or nonlinear combination of correlations in extrafoveal reach and dissociated saccade tasks.

Altogether this study shows that dorsal pulvinar contributes largely in visually-guided eye and arm movement planning with a large similarity to representations in medial intraparietal cortex (especially within each monkey). This results motivates us to further investigate causal role of pulvinar in shaping cortical neuronal representations in visually-guided reaches.

\subsection{Acknowledgments}

I especially thank Dr. Kagan for his unlimited support during this project. I thank Lukas Schneider for programming task controller and preprocessing of the behavioral as well as electrophysiological data and great scientific discussions and also Benjamin Dann and Swathi Sheshadri for their supports in LFP analysis. I also thank Adán Ulises Domínguez Vargas for collecting data from dorsal pulvinar in monkey L. I appreciate Daniela Lazzarini, Ira Panolias, Sina Plümer, Leonore Burchardt, Klaus Heisig, and Dirk Prüße and Ralf Brockhausen for their technical support. 



\section{Chapter 3}

\section{Effects of dorsal pulvinar inactivation on parietal reach and saccade signals in both hemispheres}

\subsection{Abstract}

Pulvinar is the largest thalamic nucleus and has complex and rich connectivity with many cortical areas that might play a major role in modulating the cortico-cortical and cortico-thalamo-cortical communications. In particular, dorsal aspect of the pulvinar has reciprocal connections with frontoparietal network of visuallyguided reach and saccade movement planning areas, and its lesion has been shown to cause deficits similar to deficits after parietal lesions, such as spatial neglect and optic ataxia. In addition, cells in pulvinar seem to encode visually-guided eye and arm movements, partly similar to what has been demonstrated in parietal cortex. In spite of these indications, it is still not clear whether pulvinar has a causal role in mediating neuronal activities and inter- and intra-hemispheric communication in parietal cortices during goal-directed behavior. In this study, we recorded neuronal activity simultaneously from both hemispheres in area MIP (while monkey was performing delayed visually-guided reach task) and separately in area LIP (while monkey was performing delayed saccade task), before and after unilaterally reversible pharmacological inactivation of the dorsal pulvinar in right hemisphere. We asked whether disruption of information transfer from or into pulvinar would change neuronal encoding for hand, eye and space in parietal cortex in the two hemispheres. We showed that pulvinar inactivation caused mainly contralesional hand/space behavioral deficits with more pronounced deficits in reach than in saccade task. Pulvinar inactivation strongly affected neuronal responses (both spiking and local field potential, LFP, activity) in the areas LIP and MIP. In both hemispheres, different cells exhibited decreasing and increasing firing rates after the inactivation. Population of cells in LIP in both hemispheres as well as MIP only in inactivated hemisphere showed both increase and decreasing effects. However, cells in MIP of intact hemisphere mostly increased their firings during reach task which implies a potential compensatory effect and/or reciprocal push-pull interactions between the hemispheres. Furthermore, pulvinar inactivation caused strong and consistent (across sites and sessions) changes in the LFP power in MIP and LIP, both in the inactivated and the opposite hemisphere. Reminiscent of results from few previous studies in other parts of the pulvinar and visual cortex with different tasks, low frequency power (delta-theta-alpha) in the inactivated hemisphere significantly increased (in the entire trial except movement period). At the same time, higher beta and gamma power increased in the intact hemisphere. Alpha and beta band power was modulated after inactivation in a limb-specific and hemisphere-specific manner although differently in MIP than LIP. In addition to the modulations in the power of oscillatory activity in parietal cortex, dysfunction of pulvinar disrupted coherent oscillations in theta and beta frequencies not only within inactivated hemisphere but also across two hemispheres. Altogether our data shows that pulvinar plays an important role in visually-guided motor goal behavior and it is causally contributing to shaping the activity in parietal reach and saccade movement-planning areas by modulating firing rates, oscillatory activity in both hemispheres, as well as synchronization not only within the inactivated and the intact hemispheres but also across two hemispheres. These diverse changes might reflect both induced functional impairment and compensatory mechanisms that enable the monkey to successfully perform the tasks even after the inactivation. 


\subsection{Introduction}

\subsubsection{Task and effector encoding in dorsal pulvinar and similarities with MIP}

In the previous chapter we investigated the encoding of eye, hand and space variables in cortical area medial intraparietal (MIP) and also dorsal aspect of subcortical pulvinar (dPul) in thalamus. We showed that both brain structures share similarities in their neuronal oscillations for foveal and extra-foveal reaches as well as saccades. Local field potentials in both MIP and dPul contain mainly beta band oscillations $(13-30 \mathrm{~Hz})$ but also weaker gamma $(30-120 \mathrm{~Hz})$ and theta band $(8-11 \mathrm{~Hz})$. For example, we showed that in both MIP and dPul, the decrease of beta power relative to fixation is stronger when monkey performs reaches than saccade and is stronger mostly for contralateral than ipsilateral hand.

We also showed that MIP and dPul have spatial tuning in the theta and gamma band with a tendency toward contralateral space. In addition, beta band in both regions had a general similar modulation. It was higher during fixation periods and lower during movement planning and lowest during execution. There was mainly hand tuning rather than space tuning in the beta band, with a tendency to decrease more for contralateral hand relative to fixation period, consistent with previous studies in parietal cortex which only investigated the spatial specificity of reaches with contralateral arm (Dean et al., 2012; Engel \& Fries, 2010; Stetson \& Andersen, 2014; Wong et al., 2016). And in line with the studies in which Parkinson patients who have difficulties to control their movements had higher beta band oscillations (Little \& Brown, 2014).

Although we know from many previous studies that area MIP and PRR encode spatial location of targets for arm movements stronger than saccades (which is called an arm-specific area) (Cui \& Andersen, 2007; Dean et al., 2012; Hwang \& Andersen, 2011; Snyder et al., 1997), there has been only three specific studies about reach related neuronal responses in pulvinar (Acuna et al., 1990, 1983; Cudeiro et al., 1989). They presented data in which a subset of neurons in primate pulvinar were active more when both eye and arm movements were executed than reach movement alone. From a group of reach related neurons only a small percentage was spatially tuned but all of the reach-related neurons were responsive more for contralateral arm than ipsilateral (Acuna et al., 1990).

These similarities imply that pulvinar might be actively involved in planning for visually-guided reach and saccade spatial goals. Further evidences come from lesion and inactivation studies.

\subsubsection{Similar deficits after MIP or pulvinar lesions}

Interestingly some of the deficits seen after damage to pulvinar resemble deficits after parietal lesions. For example (Christopoulos et al., 2015b) also showed that PRR inactivation causes ipsilesional choice bias for reaches but not for saccades. Several studies have shown that lesions in PRR does not strongly affect saccadic behavior but mostly influence reaching. For example some studies observed inaccuracy in reach trajectories and endpoints after PRR inactivation (Hwang et al., 2012, 2014; Yttri et al., 2014). Lesions to both pulvinar and PRR cause slower movement reaction time and movement times with contralateral arm (Wilke et al., 2018, 2010; Yttri et al., 2014).

But one evidence that differentiates pulvinar from parietal lesion symptoms is that inactivation of areas close to MIP cause misreaches which would be decreased or eliminated when subject is allowed 
to look during reaching (optic ataxia; (Andersen et al., 2014; Caminiti et al., 2010)). There is lack of evidence whether pulvinar inactivation also causes symptom. This has not been systematically tested in monkeys yet (there is ongoing work in our group that addresses inactivation effects in dissociated (extra-foveal) and free-gaze (foveal) reach tasks) but there is a recent study on a patient with bilateral pulvinar lesion which did not show similar effect (Wilke et al., 2018).

\subsubsection{Thalamocortical interactions}

We know that most of the cortical areas that have direct cortico-cortical connections are also connected with each other indirectly through thalamus (so called "replication principle", (Shipp, 2003)), which strongly suggests that thalamus might have a casual neuronal influence in their activity. It has been also shown that higher order thalamus causally modulates neuronal activity in the cortical areas related to cognitive functions, such as attention, memory (Ketz et al., 2015; Saalmann et al., 2012; Sweeney-Reed et al., 2014; Zhou et al., 2016).

Higher order thalamus, e.g. pulvinar, is reciprocally connected to cortical areas in dorsal stream of visuomotor pathways. Synchronous neuronal activities between those inter-connected regions are enhanced during cognition and suppression of thalamus decreases cognitive abilities probably through disruptions in synchronized communications (Parnaudeau et al., 2013; Purushothaman et al., 2012; Soares et al., 2004; Staudigl et al., 2012; Zhou et al., 2016).

In a recent experiment on monkeys, (Zhou et al., 2016), investigated the causal role of pulvinar in cortical visual processing. First, they simultaneously recorded from ventral pulvinar, V4 and IT and found that attention modulates neuronal responses in pulvinar although less than area V4. They also found that attention enhanced coherence not only between spikes and LFPs within V4, within IT and across V4-IT in the gamma band but also between spikes and LFP across V4-pulvinar. Second, they inactivated pulvinar and recorded from V4 and IT. Pulvinar inactivation caused contralesional attentional deficit. It increased generally lower frequency power in V4 but decreased gamma band power in attended condition. It also caused reduction of V4 cell responses to the visual stimulus as well as reduction in the V4 attentional modulation. In addition, spike-field coherence decreased after pulvinar inactivation in V4, stronger for attended condition. It reduced pulvinar-V4 Granger causality in the alpha band and reduced LFP-LFP coherence in IT as well as V4. Therefore, pulvinar inactivation not only affected within-area but also across-area neuronal communications.

According to existing evidence from anatomical studies, frontoparietal cortical areas are reciprocally connected with pulvinar (and also with each other directly and through pulvinar) (Cappe et al., 2009, 2007). This suggests that pulvinar might play an important role to mediate neuronal responses related to goal-directed behavior in cortical areas.

\subsubsection{Aims}

No study so far addressed the direct causal role of dorsal pulvinar in modulating the neuronal responses and interactions in the cortical populations which encode visually-guided reaches. Given that both dorsal pulvinar and medial intraparietal area are reciprocally connected (Cappe et al., 2007), share similarities in arm movement neuronal responses and also lesions to both cause partially similar behavioral effects, we hypothesize that pulvinar inactivation changes MIP responses in a delayed 
extra-foveal reach task. In addition, one aspect which is usually ignored is the fact that primate brain consists of two cerebral cortices and many cortical areas have their functionally similar counterpart in the other hemisphere, connected mono- or poly-synaptically through corpus callosum (CC) or other interhemispheric routes. Therefore, we were interested in understanding how unilateral pulvinar inactivation may affect responses in MIP not only within inactivated but also in intact hemisphere. We ask whether suppressing activities in one pulvinar might change the intra- and inter-hemispheric communications in a way that correlate with the behavioral deficits.

To address these questions, we trained one monkey to perform visually-guided dissociated delay saccade and reach task (with either left or right arm, interleaved). We combined reversible pharmacological inactivation and extracellular recording methods to investigate the causal role of dorsal pulvinar in parietal neuronal modulations for eye or hand movement planning and execution. We recorded neuronal activity simultaneously from both hemispheres in MIP (while monkey doing reach task) and separately in LIP (while monkey doing saccade task), before and after pharmacological inactivation of pulvinar in right hemisphere (unilaterally). We attempted to maintain stable recordings from the same neurons before and after the injection. We asked whether disruption of information transfer from/into pulvinar would change neuronal encoding for hand, eye and space in parietal cortex in the first place and if so whether those changes would be different in different hemispheres. Then we asked whether there is a correlation between behavioral deficits and the potential inactivation-induced modulations of parietal neuronal representations.

\subsection{Methods}

\subsubsection{Procedures}

All experimental procedures were conducted in accordance with the European Directive 2010/63/EU, the corresponding German law governing animal welfare, and German Primate Center institutional guidelines. The procedures were approved by the responsible government agency (Niedersaechsisches Landesamt fuer Verbraucherschutz und Lebensmittelsicherheit (LAVES), Oldenburg, Germany).

\subsubsection{Animal preparation}

One male rhesus monkeys (Macaca mulatta), monkey L weighing $\sim 10 \mathrm{~kg}$ served as subject (same monkey as in the previous chapter; See 2 Methods). In an initial surgery, the animal was implanted with an MRI-compatible polyetheretherketone (PEEK) head post embedded in a bone cement head cap (Palacos with gentamicin; BioMet) anchored by ceramic screws (Rogue Research) under general anesthesia and aseptic conditions. MR-visible markers were embedded in the head cap to aid the planning of the chamber in stereotaxic space: Monkey L, left hemisphere: center at $-3.12 \mathrm{P} / 20.2 \mathrm{~L}$ mm, tilted:-18 P/37 L degrees Monkey L right hemisphere: center at $-3.12 \mathrm{P} / 20.2 \mathrm{~L} \mathrm{~mm}$, tilted:-18 P/37 L degrees with the MR-guided stereotaxic navigation software Planner (Ohayon \& Tsao, 2012). A separate surgery was performed to implant PEEK MRI-compatible chambers (inside diameter $22 \mathrm{~mm}$ ) allowing access to the pulvinar and medial and lateral intraparietal areas (MIP and LIP respectively). After confirming chamber positioning with a postsurgical MRI, a partial craniotomy was made inside the chambers. 


\subsubsection{MR imaging}

The monkey was scanned in a 3 T MRI scanner (Magnetom TIM Trio; Siemens). Full-head T1weighted (3D magnetization-prepared rapid gradient-echo, MPRAGE, $0.5 \mathrm{~mm}$ isometric) and additional T2-weighted (rapid acquisition with relaxation enhancement, RARE, $0.25 \mathrm{~mm}$ in plane, $1 \mathrm{~mm}$ slice thickness) images with the slice package aligned to the chamber vertical axis were acquired before and after chamber implantation using the built-in gradient body transmit coil and a custom single-loop receive coil (Windmiller Kolster Scientific). In addition to preimplantation and postimplantation scans, similar T1- and T2-weighted scans were acquired periodically during the course of experiments either in an awake or sedated state to confirm electrode positioning. T1- and T2-weighted scans were coregistered and transformed into "chamber normal" (aligned to the chamber vertical axis) and to AP-PC space for electrode targeting and visualization. These images were acquired with the chamber and the grid (custom-made MR-compatible polyetherimide (Ultem)) filled with gadolinium (Magnevist; Bayer)/saline solution (proportion 1:200) with tungsten rods inserted in predefined grid locations for alignment purposes.

\subsubsection{Pulvinar and parietal targeting}

The location of the electrodes was estimated for every recording site based on anatomical MRI. Custom-made MR-compatible polyetherimide (Ultem) grids $(0.8 \mathrm{~mm}$ hole spacing, $0.45 \mathrm{~mm}$ hole diameter) were used to position electrodes in the corresponding grid hole and estimated depth. During the penetration, recording electrodes were protected using a custom-made stainless steel guide tube (450 $\mu$ m outer diameter, 27 gauge Spinocan, Braun Melsungen) which was attached to the Thomas recording drive. The tip of the guide tube aimed to land just below the dura according to the MRI images. The drug injection cannula was also protected using a same custom-made stainless steel guide tube ( $450 \mu \mathrm{m}$ outer diameter, 27 gauge Spinocan, Braun Melsungen). The tip of this guide tube aimed to land just below the dura using a stopper (530 $\mu \mathrm{m}$ inner diameter, $665 \mu \mathrm{m}$ outer diameter, 23 gauge MicroFil; World Precision Instruments). For details see chapter. 2 Fig.2.1.

\subsubsection{Electrophysiological recordings}

The electrophysiological recording was done in the same way described in 2 (see 2.3.5).

In each experimental session, we recorded neuronal signal in parietal cortex before and after the unilateral (right) pulvinar pharmacological inactivation from monkey L. In all of those sessions, monkey performed one block of trials (240 successful trials) before and more than one block after the inactivation procedure. To increase the spike sorting performance and be able to track the activity of units by time, we recorded signal also during the inactivation procedure ( 45-60 minutes) but didn't use that data for any further analysis.

\subsubsection{Reversible pharmacological inactivation}

$\mathrm{A} \mathrm{GABA}_{\mathrm{A}}$ agonist drug 4,5,6,7-tetrahydroisoxazolo[5,4-c]-pyridin-3-ol (THIP or gaboxadol) was used to suppress neuronal activity in pulvinar nucleus of the right hemisphere of monkey L. This drug seems to be a selective ligand for eGABARs (Meera et al., 2011). THIP is a derivative of alkoloid 
Muscimol (IUPAC name: 5-(Aminomethyl)-isoxazol-3-ol), which itself is a more potent $\mathrm{GABA}_{\mathrm{A}}$ agonist.

For example, (Waszczak et al., 1980) tested the effects of three GABAergic agents (Muscimol, THIP and GABA) both invivo and invitro. They reported that all three had similar inhibitory effects but Muscimol and GABA are respectively more potent than THIP in which muscimol was approximately three times more potent than THIP in inhibiting reticulata cell firing. In another study, (Kelly \& McCulloch, 1982) applied intravenously muscimol (0.15-5 mg/kg) and THIP (1-10 mg/kg) to measure cerebral glucose utilization. They found that with both drugs there is similar effect of reduced glucose utilization in all regions through CNS but muscimol was six times more potent than THIP.

In our study, the THIP infusions were performed while the animals were awake and sitting in their primate chair, with their heads restrained via implanted head posts. The injection was done by a high precision microinjection syringe pump (Harvard Apparatus, USA). A microinjection sharp-tip steel cannula (28 gauge; $60 \mathrm{~mm}$ length) was sitting inside the custom-made guide-tube such that tip of the cannula landed in the pulvinar Fig. 3.1. The injection volume across sessions was in the range of 4.0-4.5 $\mu 1$ with the rate of 0.25 ( 2 sessions) or $0.5 \mu 1 / \mathrm{min}$. In total we collected 13 inactivation sessions ( 9 before/after MIP recording and 4 before/after LIP recording).

To confirm that the drug was injected in the intended location, we scanned monkey using MRI before and after injecting a MR contrast agent gadolinium (Magnevist, Berlex Imaging, Montville, USA) diluted with saline (1:200) using similar parameters as the actual inactivation sessions $(4.0 \mu 1$ with the rate of $0.5 \mu 1 / \mathrm{min})$.

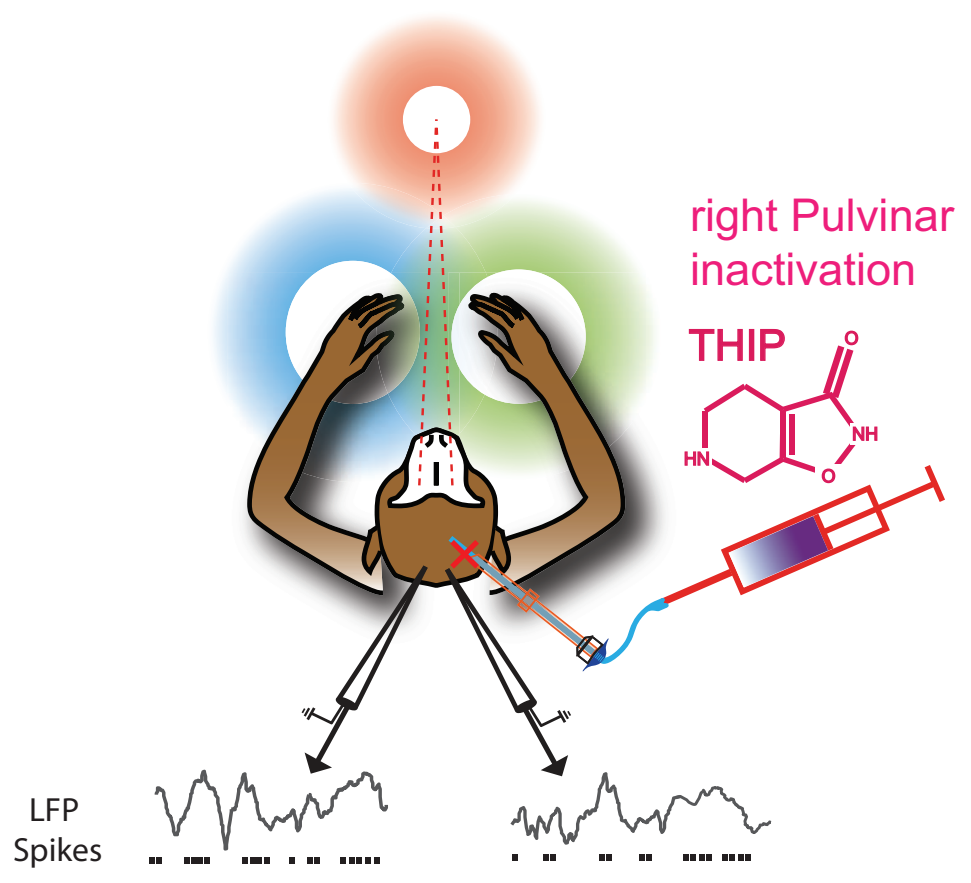

Simultaneous bihemispheric recordings from parietal areas (MIP or LIP)

Fig. 3.1 Pharmacological inactivation and simultaneous bihemispheric recording from parietal areas MIP and LIP 
Table 3.1 pulvinar inactivation

\begin{tabular}{|c|c|c|c|c|c|c|c|c|c|}
\hline Session & Hemi & $\begin{array}{l}\text { Injected } \\
\text { to }\end{array}$ & $\begin{array}{l}\text { recorded } \\
\text { from }\end{array}$ & $\begin{array}{l}\text { Grid } \\
\text { hole }\end{array}$ & $\begin{array}{l}\text { canula tip } \\
\text { from the } \\
\text { chamber } \\
\text { top }\end{array}$ & Drug & $\begin{array}{l}\text { Dose } \\
\text { (ul) }\end{array}$ & $\begin{array}{l}\text { Concentration } \\
(\mathrm{mg} / \mathrm{ml})\end{array}$ & $\begin{array}{l}\text { Speed of } \\
\text { injection } \\
(\mathrm{ul} / \mathrm{min})\end{array}$ \\
\hline 1 & right & PUL & MIP & $(-1,3)$ & 45 & THIP & 4.5 & 6.6 & 0.5 \\
\hline 2 & right & PUL & MIP & $(-1,3)$ & 45 & THIP & 4.5 & 6.6 & 0.5 \\
\hline 3 & right & PUL & MIP & $(-1,3)$ & 45 & THIP & 4.6 & 6.6 & 0.5 \\
\hline 4 & right & PUL & MIP & $(-1,3)$ & 45 & THIP & 4.6 & 6.6 & 0.5 \\
\hline 5 & right & PUL & MIP & $(-1,3)$ & 45 & THIP & 4.5 & 6.6 & 0.5 \\
\hline 6 & right & PUL & MIP & $(-1,3)$ & 44 & THIP & 4.0 & 6.6 & 0.5 \\
\hline 7 & right & PUL & MIP & $(-1,3)$ & 44 & THIP & 4.1 & 6.6 & 0.5 \\
\hline 8 & right & PUL & MIP & $(-1,3)$ & 45 & THIP & 4.0 & 6.6 & 0.5 \\
\hline 9 & right & PUL & MIP & $(-1,3)$ & 46 & THIP & 4.0 & 6.6 & 0.5 \\
\hline 10 & right & PUL & LIP & $(-1,3)$ & 46 & THIP & 4.0 & 6.6 & 0.5 \\
\hline 11 & right & PUL & LIP & $(-1,3)$ & 45 & THIP & 4.0 & 6.6 & 0.5 \\
\hline 12 & right & PUL & LIP & $(-1,3)$ & 45 & THIP & 4.0 & 6.6 & 0.5 \\
\hline 13 & right & PUL & LIP & $(-1,3)$ & 45 & THIP & 4.0 & 6.6 & 0.5 \\
\hline
\end{tabular}

\section{Monkey L right hemisphere Pulvinar THIP injection setup}

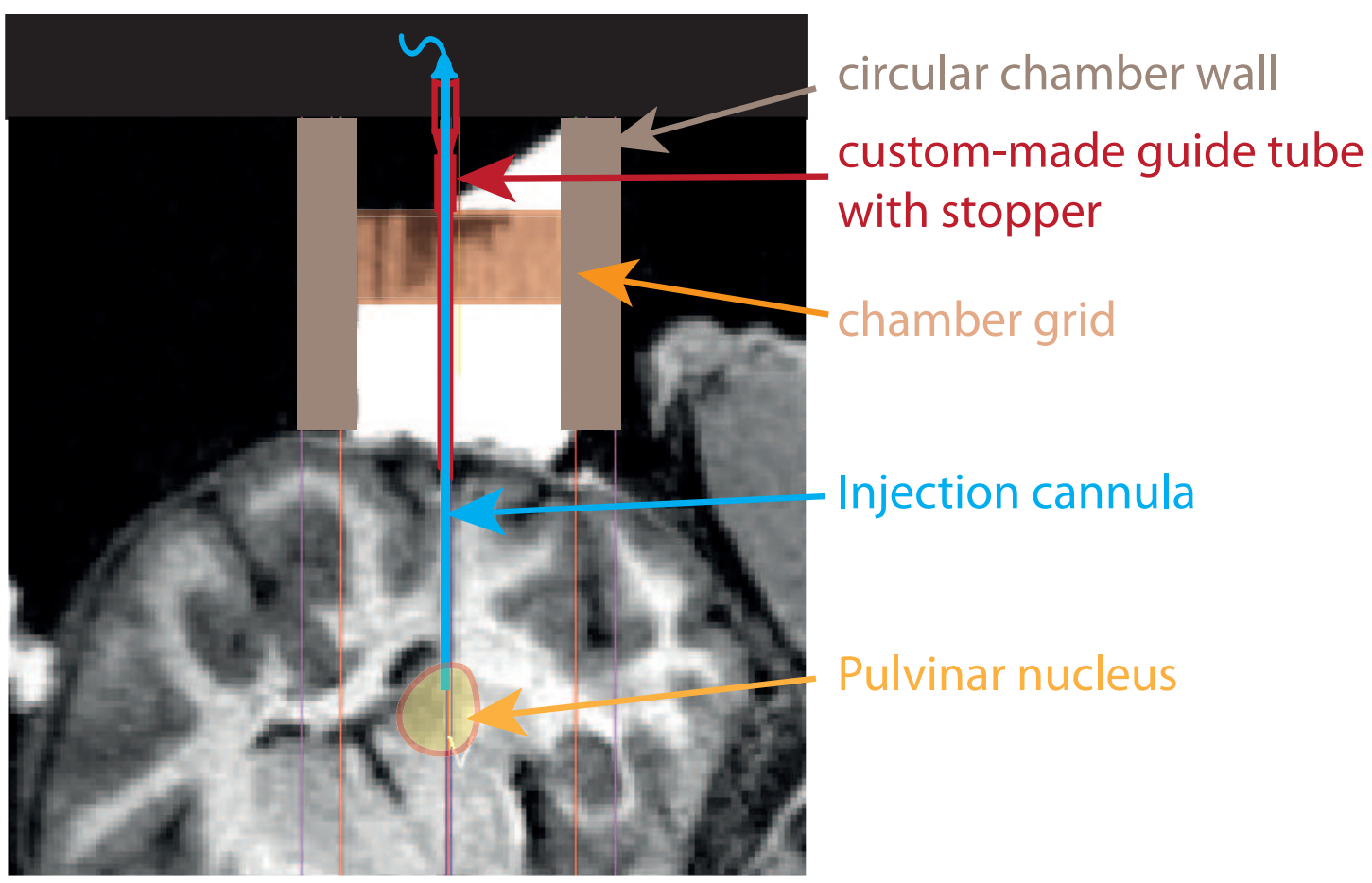

Fig. 3.2 Scheme for the drug injection 


\subsubsection{Behavioral paradigm}

This project consists of two parts: a) 9 sessions of recording from medial intraparietal area (MIP) while inactivating pulvinar, in which monkey performed only Dissociated delay reach (Ddr) task; b) 4 sessions of recording from lateral intraparietal area (LIP) while inactivating pulvinar in which monkey executed only dissociated delay saccade (Dds) task. Both tasks were described in the previous chapter (see chapter 2 Methods).

\subsubsection{Data analysis}

All behavioral as well as neuronal signals were analyzed in MATLAB (version R2011b or R2012b, The MathWorks, Inc., USA).

\section{Saccade and reach definition}

This was defined in the same way as described in the previous chapter (see chapter. 2 methods).

\section{Local field potentials}

We recorded in total 85 LFP sites from MIP (43 right and 42 left hemisphere) and 39 sites in LIP (20 from right and 19 left hemisphere). LFP signal power and synchrony measures were computed using Fieldtrip toolbox (Oostenveld et al., 2011). LFP power was obtained for each of the frequency bins between 2 and $120 \mathrm{~Hz}$ in logarithmic steps ("logscale" function of MATLAB) by applying Hanning window taper Fourier transform. To get the power time-frequency representation (TFR or spectrogram), we used a cycle-based time window for each frequency ( $\mathrm{N}=4$ cycles) with the step of $50 \mathrm{~ms}$ through one trial. This means for lower frequencies the time window was longer than the higher frequencies. Since for a typical length of trial (about 6 seconds), the full power distribution for the whole trial in lower frequencies was not possible, zero-padding was done such that the length of a trial was enough for power calculations for all frequency bins.

We also computed the average LFP power for separate frequency-bands (Delta [2 4) Hz, Theta [4 8) Hz, Alpha [8 12) Hz, Beta1 [12 18) Hz, Beta2 [18 30) Hz, Gamma1 [30 70) Hz, Gamma2 [70 120) $\mathrm{Hz}$ ), by averaging the power across all frequency bins within each band.

We normalized spectrograms within each session for the good of following reasons: to bring all frequencies to a comparable level (to account for $1 / \mathrm{f}$ nature of power spectrum) and also to be able to compare hand-space representations across blocks and sessions. Toward this, LFP power in each frequency was normalized by z-score approach: $P(f t)=(P(f t))-$ mean $(P)) / s t d(P)$. The statistical measures (mean and standard deviation) were computed across all successful trials of the first Ddr task in a session (we took Ddr task as our reference for the comparisons). With this procedure we were able to investigate task selectivity as well as the hand-space (condition) selectivity of the LFP power.

We only analyzed a trial if it was not detected as noisy. To detect noisy trials, we computed first the raw LFP power spectrogram and then mean and standard deviation of power across time-bins within each frequency. If at any time bin more than $50 \%$ of the frequency bins had power more than 
mean $(f))+2 * s t d(f)$, that trial detected as noisy. This is because we observed that spurious body movements caused sharp broad-band (across many frequencies) increase in power.

\section{Single/Multi unit activity}

We recorded in total 74 units in MIP (37 left and 37 right hemisphere) and 50 units from LIP (23 left and 27 right hemisphere). Spike density function for each trial was calculated using Fieldtrip toolbox by running a Gaussian kernel with a standard deviation of $25 \mathrm{~ms}$ for a window size of $100 \mathrm{~ms}$, sampled each $2 \mathrm{~ms}$. To evaluate how much pulvinar inactivation modulates firing rates (FR) of units in parietal area MIP, we calculated inactivation modulation index (IMI) for each unit, each task period and each condition separately: IMI= (average FR in post injection trials - average FR in pre injection trials)/ (average FR in post injection trials + average FR in pre injection trials).

We computed IMI for the following periods: fixation hold (300-0 ms before cue presentation), cue (50-150 ms after cue presentation) and pre-reach (300-10 ms before movement onset) and following conditions: ipislesional hand, ipsilesional space, contralesional hand, contralesional space.

\section{Statistics}

To test the behavioral effects of inactivation we used mostly paired ttest across sessions (pre- versus post-injection block). We calculated behavioral measures in each block of each session for example reaction time, movement time, success rate, choice bias and the performance on choices. Then we applied paired ttest to compare pre versus post injection effects. In addition, we compared the condition specific effects (e.g. ipsilesional versus contralesional) also across sessions in a paired-wise manner to answer the question whether the difference between pre- versus post-injection measures was different for ipsi- compared to contralesional conditions.

To compare if there is significant difference between contra versus ipsi-lateral hand or space representations on the population level, we applied paired ttest across sites with FDR multiple comparison correction ((Benjamini \& Hochberg, 1995); matlab function "fdr_bh" by David Groppe) to reject false discovery for each time-frequency bin. In each bin with a significant difference we show the actual normalized power value and in the rest the value set to zero. In other words, we show the strength of the power only for the bins with a significant difference.

To compare power spectrograms between two tasks or areas, we did the same analysis. We applied two-sample ttest on each bin across LFP sites for two cases and used FDR method (False discovery rate) to adjust the p-value for the significance level. But to reduce the complexity, we plotted also effect-size map which is the averaged power across bins for each frequency band and five epochs (in total a $5 * 7$ colormap matrix). The difference between two cases was defined as the population effect size. If the same sites contributed to the two cases (e.g. when comparing across tasks), effect size computed as mean across differences, otherwise it was computed as difference of means. To test if this population effect size was significant in each epoch-frequency combination, we calculated ttest (paired or non-paired) and marked the non-significant pixels with a circle. We used the following frequency bands: delta $[24) \mathrm{Hz}$, theta $[48) \mathrm{Hz}$, alpha $[813) \mathrm{Hz}$, beta1 $[1318) \mathrm{Hz}$, beta2 $[1830) \mathrm{Hz}$, gamma1 $[3070) \mathrm{Hz}$, gamma2 [70 120)Hz and following epochs: fxh (500-0 ms before cue), cue 
(0-300 after cue), del (600-1000 ms after cue), tgo (400-0 ms before movement onset), mvm (0-400 after movement onset).

To observe also the effect size on the single site level (scatter plots), we plot the z-scored power of each site in one case versus the other for each epoch and frequency separately. Here we mentioned the same statistics as in the effect size plots.

To test whether the firing rate of units significantly changed after inactivation, we applied unpaired ttest on the actual firing rates, in a certain epoch and in a certain condition, between all successful trials in the pre-injection state versus post-injection state.

\subsection{Results}

\subsubsection{Behavioral effects of unilateral pulvinar inactivation in the delay reach task}

In the following subsections we show how the unilateral pulvinar inactivation affected behavioral parameters of the monkey. As explained before monkey performed one block of successful trials $(n: 240)$ before the inactivation and at least one block after the inactivation. In most of the sessions monkey performed two blocks. Since the behavioral effects were stronger in the second than the first block, here we will show only the effects on the second block of trials. We grouped trials either by hand (contra- vs ipsi-hand) or space (contra- vs ipsi-space) or the combination of those.
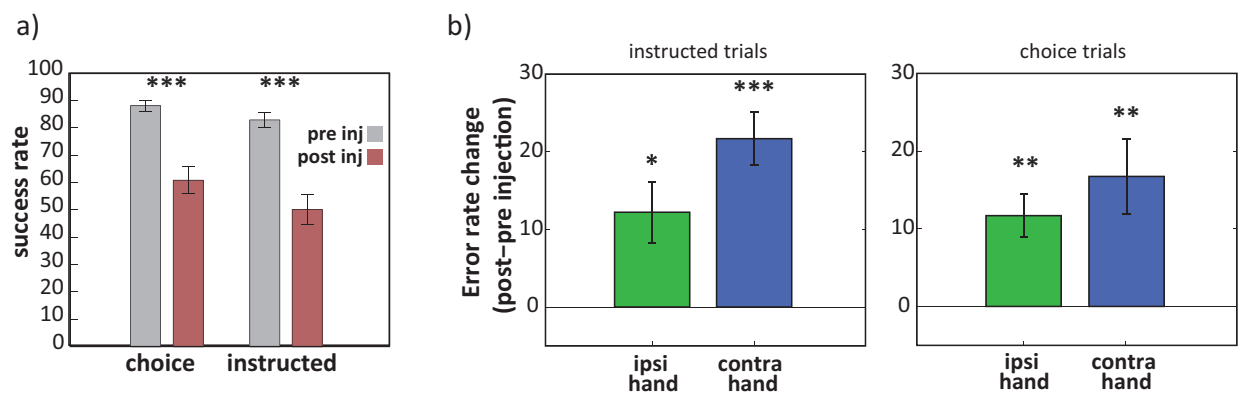

Fig. 3.3 a) Overall performance for pre- (gray) versus post-injection (red) trials, separately for instructed and choice trials. b) Change in performance after inactivation for each hand, and for instructed and choice trials separately. Errorbars are standard error of mean. Significance was computed by paired ttest: $*(\mathrm{p}<0.05) * *(\mathrm{p}<0.01) * * *(\mathrm{p}<0.001)$

\section{Success rate}

Monkey's performance significantly dropped after pulvinar inactivation in both instructed (preinjection $\mathrm{M}=88.2 \% \mathrm{SEM}=2 \%$ vs. post-injection $\mathrm{M}=61 \% \mathrm{SEM}=5 \%$; paired ttest $\mathrm{p}=0.0009$ ) and choice trials (pre-injection $\mathrm{M}=83 \% \mathrm{SEM}=2.6 \%$ vs. post-injection $\mathrm{M}=50.2 \% \mathrm{SEM}=5.5 \%$; paired test p=0.0004; Fig. 3.3(a)). Fig. 3.3(b) illustrates that error rate increased for both ipsi- and contralesional hand but non-significantly stronger with the contralesional hand in both instructed (ipsi hand: $M=12.2$ $\mathrm{SEM}=3.9$ paired ttest $\mathrm{df}=8 \mathrm{p}=0.02$ and contra hand: $\mathrm{M}=21.7 \mathrm{SEM}=3.4$ paired ttest $\mathrm{df}=8 \mathrm{p}=0.0003$ ) and choice trials (ipsi hand: $\mathrm{M}=11.7 \mathrm{SEM}=2.7$ paired ttest $\mathrm{df}=8 \mathrm{p}=0.004$ and contra hand: $\mathrm{M}=16.7$ SEM $=4.8$ paired ttest $\mathrm{df}=8 \mathrm{p}=0.01$ ) 


\section{Target selection}

In the pre-injection block monkey performed similar percentage of instructed trials across hand-space conditions (Fig. 3.4(a) left; CHCS(M=45.5, SEM=1.9), CHIS(M=54.5, SEM=1.9), IHCS(M=52.3, $\mathrm{SEM}=0.7)$, IHIS(M=47.7, SEM=0.7)). For those trials monkey's performance was over $75 \%$ (Fig. 3.4(a) right; $\mathrm{CHCS}(\mathrm{M}=89.0$, SEM=2.3), $\mathrm{CHIS}(\mathrm{M}=75.3$, SEM=4.6), IHCS(M=85.6, SEM=2.6), IHIS(M=93.9, SEM=2.1)). In choice trials, monkey had a strong bias to select a target which was in the same side as the involved hand (Fig. 3.4(b) left; $\operatorname{CHCS}(\mathrm{M}=86.9, \mathrm{SEM}=4.2)$, CHIS(M=13.1, SEM=4.2), IHCS(M=11.3, SEM=4.8), IHIS(M=88.7, SEM=4.8)). Monkey's overall performance on selected targets was similar to instructed trials. The lowest performance was for the CHIS condition, as in the instructed trials (Fig. 3.4(b) right; CHCS(M=90.1, SEM=1.8), CHIS(M=45.9, SEM=15.4), $\operatorname{IHCS}(\mathrm{M}=82.2, \mathrm{SEM}=12.3)$, IHIS(M=92.1, SEM=2.3)).
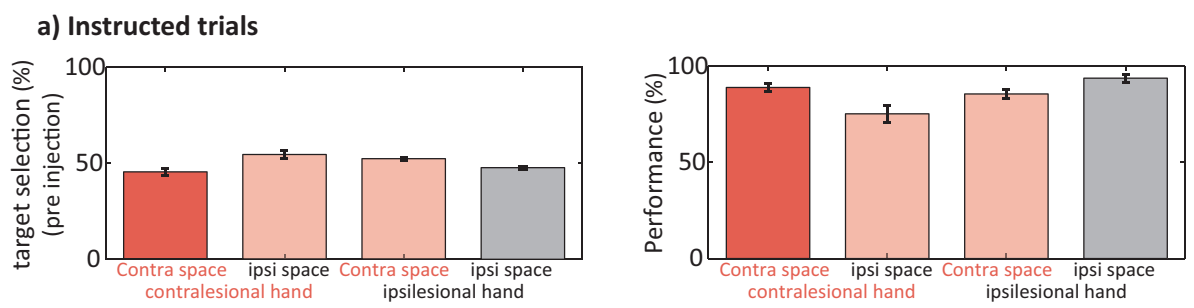

b) Choice trials
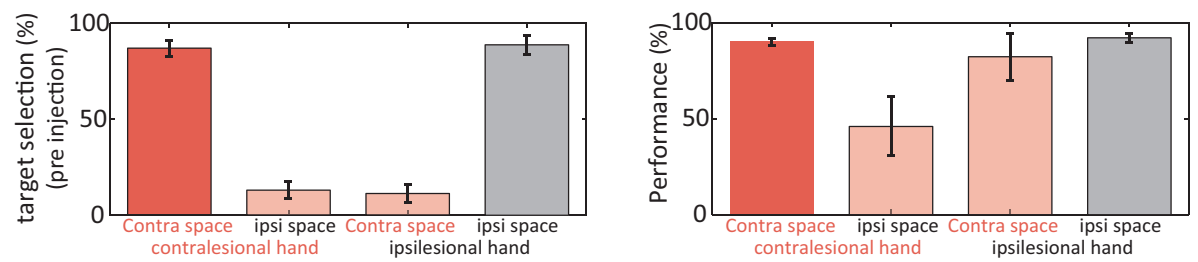

Fig. 3.4 Percentage of target selection and the performance in the pre-injection block for each hand and space combination, a) for instructed trials and b) for choice trials. Errorbars are SE of means across sessions.

Fig. 3.5 illustrates the changes in the target selection and performance in the second block of trials after pulvinar inactivation compared to pre-injection block. Across all sessions monkey selected less contralesional targets in choice trials (Fig. 3.5 $\mathrm{M}=-3.3, \mathrm{SEM}=4.4$, $\mathrm{df}=8, \mathrm{p}=0.5$ ). His performance on both target locations significantly decreased, more for ipsilesional targets (contralesional targets: $\mathrm{M}=-12.3, \mathrm{SEM}=4.7, \mathrm{df}=8, \mathrm{p}=0.04$; ipsilesional targets: $\mathrm{M}=-28.4, \mathrm{SEM}=7.3, \mathrm{df}=8, \mathrm{p}=0.006$ ).

Then we asked how these effects are hand-specific. The effect, for both target selection and selection performance, was quite similar across hands. In choice trials monkey selected significantly more ipsilesional targets with contralesional hand after inactivation, but oppositely with ipsilesional hand (not significant) (CHCS: $\mathrm{M}=-12.9, \mathrm{SEM}=4.6, \mathrm{df}=8, \mathrm{p}=0.3$; IHCS $\mathrm{M}=4.8, \mathrm{SEM}=6.2, \mathrm{df}=8$, $\mathrm{p}=0.5$ ). In addition, monkey's selection performance in choice trials decreased in all conditions but only significant for ipsilesional hand ipsilesional space (CHCS M=-11.3, SEM=5.0, $\mathrm{df}=8, \mathrm{p}=0.07$; CHIS $M=-17.1, \mathrm{SEM}=11.5, \mathrm{df}=8, \mathrm{p}=0.3$; IHCS $\mathrm{M}=-9.8, \mathrm{SEM}=21.2, \mathrm{df}=8, \mathrm{p}=0.8$; IHIS $\mathrm{M}=-23.2$, $\mathrm{SEM}=8.0, \mathrm{df}=8, \mathrm{p}=0.03)$. 


\section{Reaction and Movement time}

Reversible pharmacological right pulvinar inactivation but not affected the target selection but also the reaction time as well as the movement time of the monkey in two epochs of the task. In a dissociated reach task monkey have to start the trial by releasing only the required hand from the resting sensor and move that arm toward the fixation spot on the middle of the touch screen (fixation acquire time and the sensor release time). Besides that, later in trial monkey acquires a spatial target in the periphery (target reaction time and target movement time).

In the second block after inactivation monkey released the hands slower from the resting sensors with both hands but only significant for the contralesional hand (Fig. 3.6(a); instructed contralesional hand $\mathrm{M}=49.1 \mathrm{~ms}, \mathrm{SEM}=12.6 \mathrm{~ms}, \mathrm{df}=8, \mathrm{p}=0.006$ ). In addition, only with the contralesional hand monkey was significantly slower to acquire the fixation spot (Fig. 3.6(b); instructed contralesional hand $\mathrm{M}=30.2 \mathrm{~ms}, \mathrm{SEM}=8.5 \mathrm{~ms}, \mathrm{df}=8, \mathrm{p}=0.01$ ). These effects were very similar across instructed and choice trials.
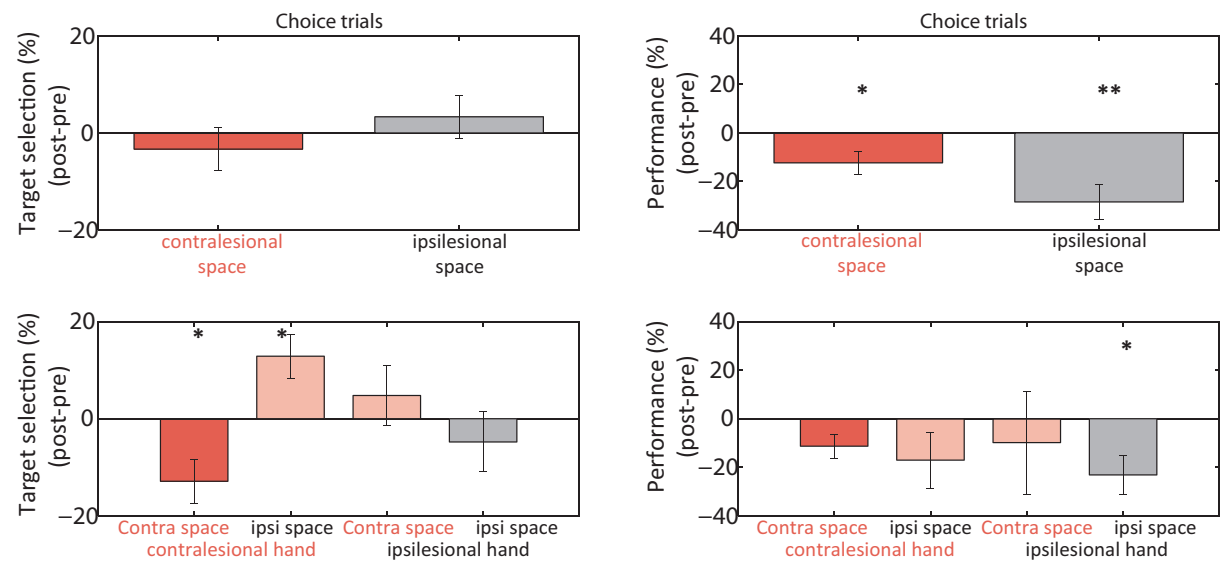

Fig. 3.5 Percentage change in target selection after pulvinar inactivation in choice trials. Positive mean increase and negative means decrease of selection after inactivation. Significance computed by paired ttest between pre and post-injection averaged across trials: $*(\mathrm{p}<0.05) * *(\mathrm{p}<0.01) * * *(\mathrm{p}<0.001)$. Contra and ipsi are relative to the inactivated hemisphere (right). In choice trials target selection refers to how many times monkey selected one of the bilaterally presented targets. 


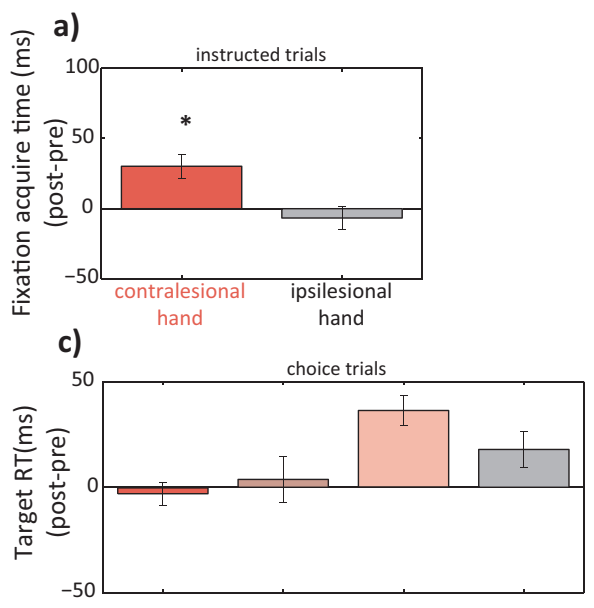

e)

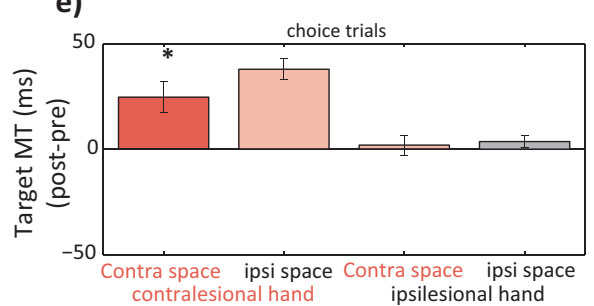

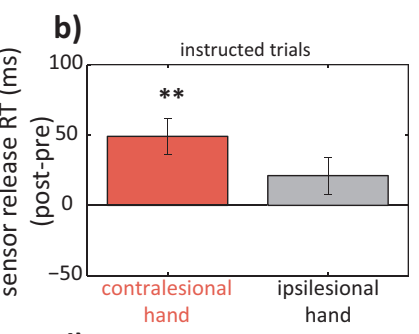
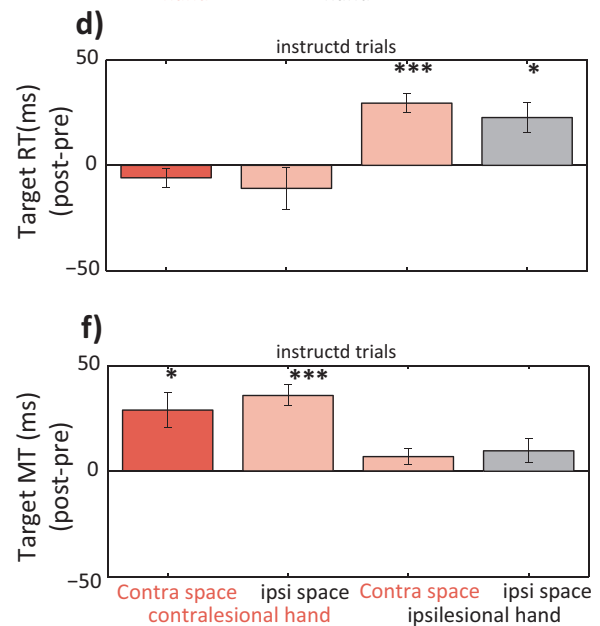

Fig. 3.6 Effects of unilateral pulvinar inactivation on the reach latency and duration: a) reach duration from proximity sensor to the fixation spot on the touch screen. b) reaction time to release required hand from sensor. c) and d) reaction time to acquire a spatial target, respectively for choice and instructed trials. e) and f) reach duration from fixation toward spatial target in choice and instructed trials respectively. Significance checked by applying paired ttest between pre and post-injection averaged across trials: $*(\mathrm{p}<0.05) * *(\mathrm{p}<0.01) * * *(\mathrm{p}<0.001)$

Surprisingly, in both instructed and choice trials, target RT didn't significantly change for reaches with contralesional hand but only with the ipsilesional hand (Fig. 3.6(c) choice: CHCS M=$3.1 \mathrm{~ms}, \mathrm{SEM}=5.6 \mathrm{~ms}, \mathrm{df}=8, \mathrm{p}>0.05$; CHIS $\mathrm{M}=3.7 \mathrm{~ms}, \mathrm{SEM}=10.8 \mathrm{~ms}, \mathrm{df}=2, \mathrm{p}>0.05$; IHCS $\mathrm{M}=36.4 \mathrm{~ms}$, $\mathrm{SEM}=7.2 \mathrm{~ms}, \mathrm{df}=2, \mathrm{p}>0.05$; IHIS $\mathrm{M}=17.9 \mathrm{~ms}, \mathrm{SEM}=8.6 \mathrm{~ms}, \mathrm{df}=8, \mathrm{p}>0.05$ and Fig. $3.6(\mathrm{~d})$ instructed: CHCS $M=-5.9 \mathrm{~ms}$ SEM=4.4ms, $d f=8, p>0.05$; CHIS $M=-10.9 \mathrm{~ms}, \mathrm{SEM}=10.1 \mathrm{~ms}, \mathrm{df}=8, \mathrm{p}>0.05$; IHCS $\mathrm{M}=29.5 \mathrm{~ms}, \mathrm{SEM}=4.7 \mathrm{~ms}, \mathrm{df}=8, \mathrm{p}=0.0003$; IHIS $\mathrm{M}=22.7$, SEM=6.9ms, $\mathrm{df}=8, \mathrm{p}=0.015$ ).

The effect on the target MT was in opposite to the RT for both choice and instructed trials (Fig. 3.6(e) and (f) respectively). Monkey was significantly slower to move the contralesional arm toward the spatial target but not the ipsilesional hand (choice: CHCS $M=24.6 \mathrm{~ms}, \mathrm{SEM}=7.3 \mathrm{~ms}, \mathrm{df}=8$, $\mathrm{p}=0.01$; CHIS $\mathrm{M}=37.9 \mathrm{~ms}, \mathrm{SEM}=4.9 \mathrm{~ms}, \mathrm{df}=2, \mathrm{p}>0.05$; IHCS $\mathrm{M}=1.9 \mathrm{~ms}, \mathrm{SEM}=4.7 \mathrm{~ms}, \mathrm{df}=2, \mathrm{p}>0.05$; IHIS $M=3.5 \mathrm{~ms}, S E M=2.9 \mathrm{~ms}, \mathrm{df}=8, \mathrm{p}>0.05$ ) (instructed: CHCS $\mathrm{M}=29 \mathrm{~ms}, \mathrm{SEM}=8.1 \mathrm{~ms}, \mathrm{f}=8, \mathrm{p}=0.01$; CHIS $M=35.9 \mathrm{~ms}, S E M=5 \mathrm{~ms}, \mathrm{df}=8, \mathrm{p}=0.0001$; IHCS $\mathrm{M}=7 \mathrm{~ms}, \mathrm{SEM}=3.8 \mathrm{~ms}, \mathrm{df}=8, \mathrm{p}>0.05$; IHIS $\mathrm{M}=9.7 \mathrm{~ms}$, SEM=5.8ms, $\mathrm{df}=, \mathrm{p}>0.05$ ).

In summary, monkey showed severe impairment in executing arm movement with contralesional hand in instructed and choice trials and to both sides of space. Also his arm reaction times toward spatial targets increased only with ipsilesional hand to both ipsi- and contralesional space. 


\subsubsection{Unilateral pulvinar inactivation alters single/multi-unit activities in area MIP}

We recorded single/multi units (74: 37 left hemisphere and 37 right hemisphere) from area MIP of both hemispheres before and after unilateral right pulvinar inactivation in monkey $\mathrm{L}$ in 9 sessions. We ask whether pulvinar inactivation changes firing pattern of the neurons in MIP in a condition specific manner and whether the potential changes are different in opposite hemispheres. In the following subsections we will show two example units and how they were affected after inactivation. In addition, we will show the effects on the population of units in each hemisphere and discuss the effects in terms of degrees of modulations in each hemisphere in different task conditions and different epochs.

\section{Examples units}

Figure 3.7 and 3.8 illustrate two units in the right (inactivated) hemisphere MIP and how they were affected after right pulvinar inactivation. Each figure is divided into four panels illustrating fours task conditions relative to the lesioned hemisphere (ipsi-hand ipsi-space, ipsi-hand contra-space, contra-hand ipsi-space, contra-hand contra-space). In each panel we show the averaged and standard deviation of firing rate aligned to the cue presentation across all successful trials in one block (preinjection and post-injection). In the bottom of each panel we show also spike rasters in each trial. Both units have peak of their response during the arm movement: one is from resting position toward the fixation spot and the other is during the movement toward the spatial target. While the unit in Fig. 3.7 has a strong cue response (stronger for the contralesional arm condition) in the pre-injection trials, the unit in Fig. 3.8 has a very small cue response. But pulvinar inactivation affected these two units differently. In the first example, the firing rate during movement periods decreased significantly (two-way ANOVA for epoch and injection block) mostly in the ipsilesional hand and contralesional space conditions. This effect was similar for the late delay period before the go signal. But in the second example, the firing rate of the unit generally increased after the inactivation. This increase is more during the arm movement execution in all conditions but slightly more when the monkey used his contralesional arm. 
Unit “Lin_20170720_13” right MIP (Ddr task)
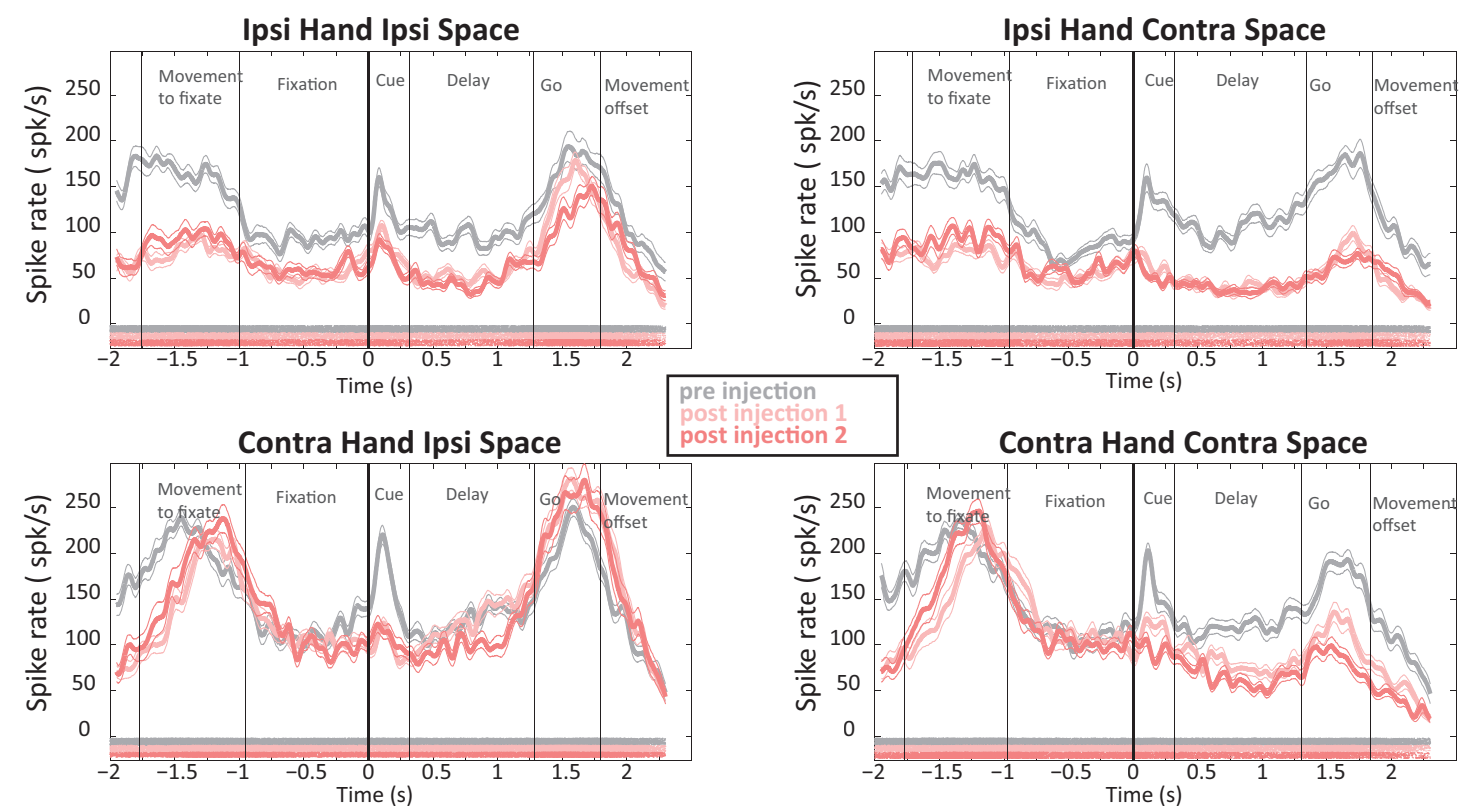

Fig. 3.7 Effect of pulvinar inactivation on the firing rate of an example unit in right MIP. Each panel illustrates effect in one task condition. All PSTHs are aligned to the cue presentation (time zero). Each color represents averaged aligned PSTH across successful trials in one block (gray: pre injection block (control), light red: first block after inactivation, dark red: second block after inactivation). Ipsi and Contra are relative to the inactivated hemisphere (right).

Unit “Lin_20170818_11" right MIP (Ddr task)
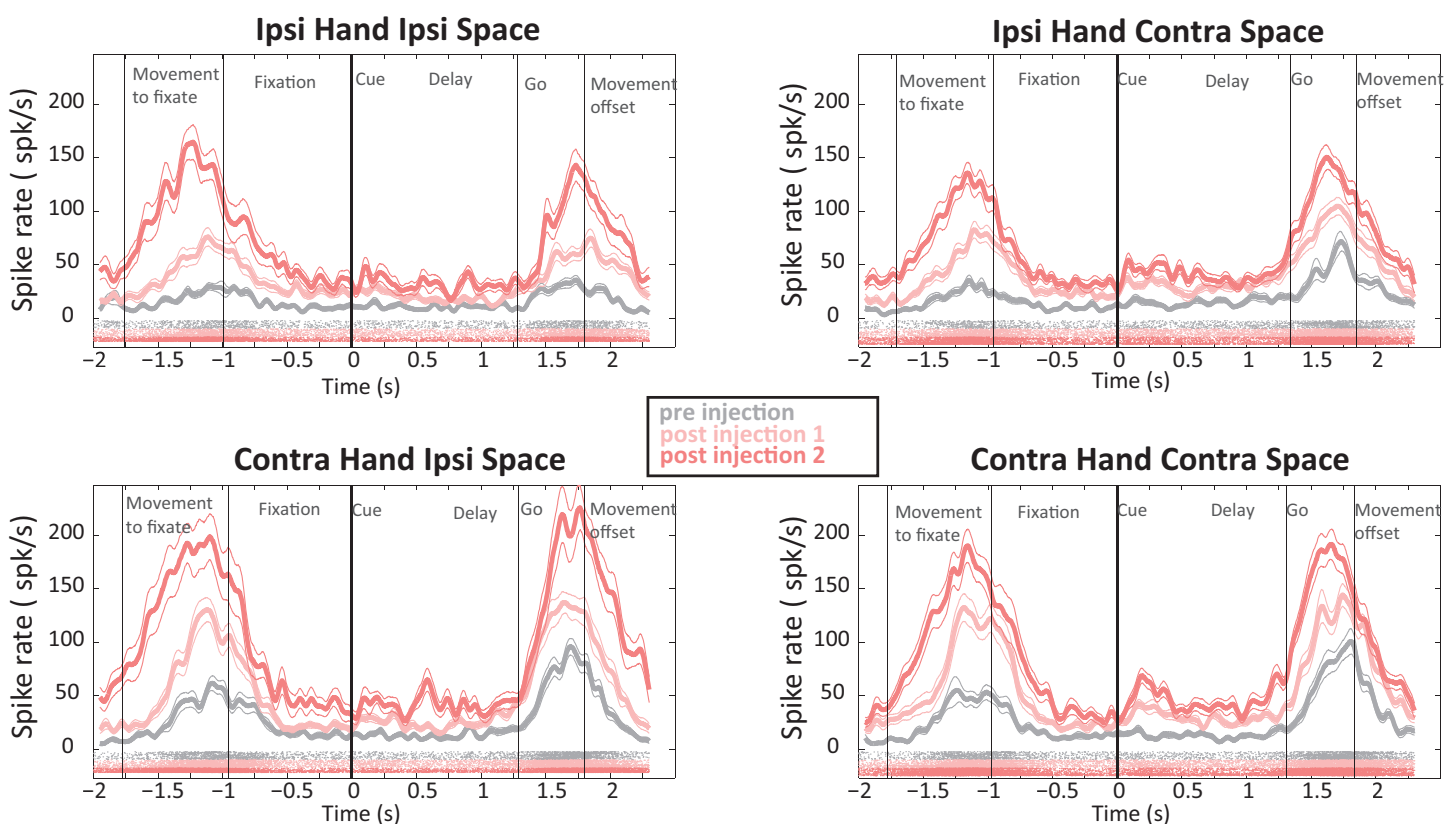

Contra Hand Contra Space

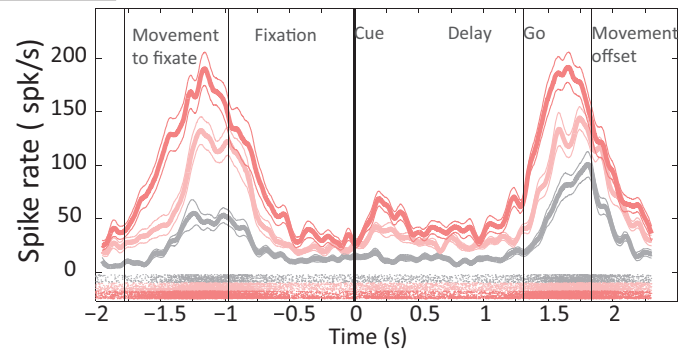

Fig. 3.8 Effect of pulvinar inactivation on the firing rate of an example unit in right MIP. Similar to Fig. 3.7. 


\section{Effects of inactivation on the population of MIP units}

To assess the effects of the pulvinar inactivation on the parietal firing rates we calculated inactivation modulation index (IMI) for separate conditions and epochs in its classical way (See Methods). Negative IMI values indicate decrease of firing rate and positive IMI values means increase of firing rate after inactivation. We computed IMI for all recorded units in the right MIP ( $n=37)$ and left MIP $(n=37)$ which had stable firing pattern through the course of recording across pre- and post-injection blocks of experiment.

Figure 3.9 shows the results of this analysis for the population of units in right hemisphere. In the fixation hold period and in all conditions mean IMI value across population was slightly negative but in none of the conditions was significant ( $p>0.05$; mean IMI: IHIS=-0.06, IHCS $=-0.08$, CHIS=-0.07, $\mathrm{CHCS}=-0.06$ ). In addition, mean IMI value across only the units with significant modulation was also slightly negative but not significant ( $p>0.05$; mean IMI: IHIS $(n=28)=-0.07, \operatorname{IHCS}(n=30)=-0.08$, CHIS(n=29)=-0.09, CHCS(n=27)=-0.08). We observed very similar effect for cue and pre-reach epochs. In the cue period overall slight decrease of firing rate but not significant on the population level ( $p>0.05$; mean IMI: IHIS $=-0.08$, IHCS $=-0.07$, CHIS $=-0.04$, CHCS $=-0.06$ ). And it was similar for only significant units ( $>0.05$; mean IMI: IHIS $(n=23)=-0.13$, IHCS $(n=22)=-0.13, \operatorname{CHIS}(n=25)=-$ 0.06 , CHCS $(n=24)=-0.09)$. During the pre-reach condition and only in the contralesional hand ipsilesional space (CHIS) the IMI for the population $(n=37)$ gets slightly significant $(\mathrm{p}>0.05$; mean IMI: IHIS $=-0.06$, IHCS $=-0.08, \mathrm{CHIS}=-0.06, \mathrm{CHCS}=-0.03$ ) but IMI across only significant units did not significantly deviate from zero ( $\mathrm{p}>0.05$; mean IMI: IHIS $(\mathrm{n}=26)=-0.08, \operatorname{IHCS}(\mathrm{n}=26)=-0.12$, CHIS $(n=25)=-0.08, \operatorname{CHCS}(n=26)=-0.04)$.

In general, we observe that pulvinar inactivation significantly modulate firing rate of many neurons in the parietal area MIP either by increasing or decreasing the rates. Overall there were more cells with decreasing firing rate than increasing but it is not significant at the population level.

But interestingly in the intact hemisphere we saw a completely different effect. We did the same analysis for the population of units in the left hemisphere $(n=37)$. In the fixation hold period, firing in most of the cells increased after pulvinar inactivation which resulted in mostly positive IMI values (Fig. 3.10). The mean IMI value across all units was highly significantly positive $(\mathrm{P}<0.001$; mean IMI: IHIS $=0.21$, IHCS $=0.23$, CHIS $=0.22, \mathrm{CHCS}=0.21$ ). Similar result holds for units with significant modulation $(\mathrm{p}<0.001$; mean IMI: IHIS $(\mathrm{n}=26)=0.28, \operatorname{IHCS}(\mathrm{n}=27)=0.30, \operatorname{CHIS}(\mathrm{n}=29)=0.28$, $\operatorname{CHCS}(n=29)=0.26)$.

Also during cue and pre-reach epochs most of the cells showed up-modulation of firing rates after pulvinar inactivation (cue p $<0.001$ mean IMI: IHIS $=0.19$, IHCS $=0.17, \mathrm{CHIS}=0.19, \mathrm{CHCS}=0.17$; and pre-reach $\mathrm{p}<0.001$ mean IMI: IHIS $=0.19$, IHCS $=0.16$, CHIS $=0.16, \mathrm{CHCS}=0.16$ ). This effect got stronger when we take only cells with significant modulation (cue $\mathrm{p}<0.001$ mean IMI: $\operatorname{IHIS}(\mathrm{n}=21)=0.32, \operatorname{IHCS}(\mathrm{n}=22)=0.27, \operatorname{CHIS}(\mathrm{n}=22)=0.30, \operatorname{CHCS}(\mathrm{n}=26)=0.24$; and pre-reach $\mathrm{p}<0.001$ mean IMI: $\operatorname{IHIS}(n=23)=0.30, \operatorname{IHCS}(n=28)=0.21, \operatorname{CHIS}(n=23)=0.24, \operatorname{CHCS}(n=26)=0.21)$.

Overall effect across epochs and conditions was similar in left (contralesional or intact) hemisphere. In the left hemisphere most of the cells (60-75\%) show significant change in their firing rate after pulvinar inactivation and from those over $85 \%$ had increase of firing. During cue period, mean IMI across units with significant modulation was higher for ipsilesional space than contralesional space ( 0.31 versus 0.25 respectively). During pre-reach epoch the highest IMI value was for the 
ipsilesional hand and space condition compared to other conditions but here also mean IMI was higher for ipsilesional space than contralesional. In this period modulation index across population was higher for ipsilesional hand than contralesional hand. This means that within the same population of cells, firing increased more for the ipsilesional conditions than contralesional. 
Inactivation modulation index (IMI) for the units in right MIP ( $n=37)$

a) Fixation hold period

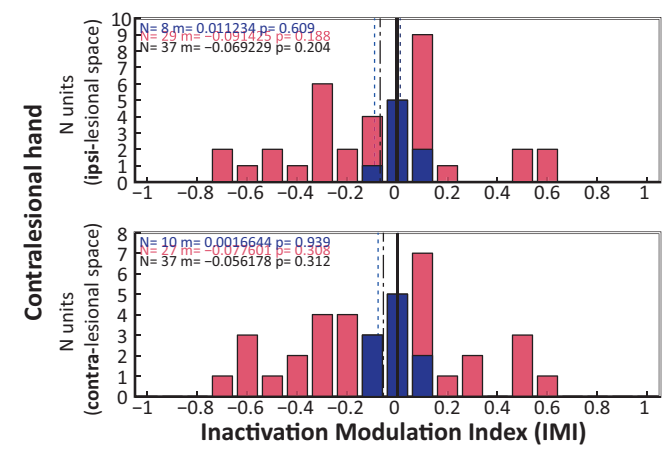

b) Cue period

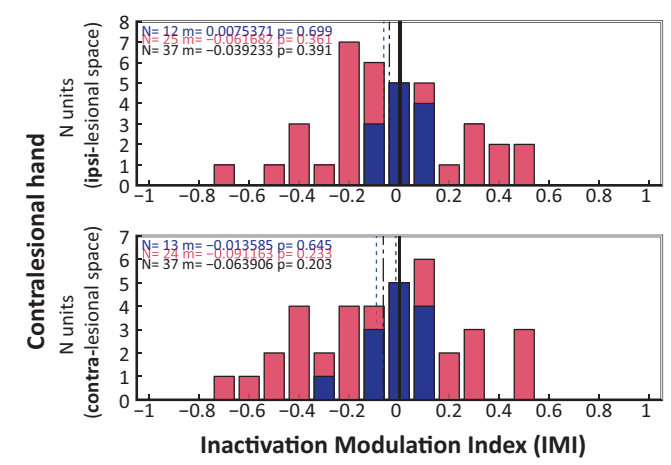

c) pre-reach period

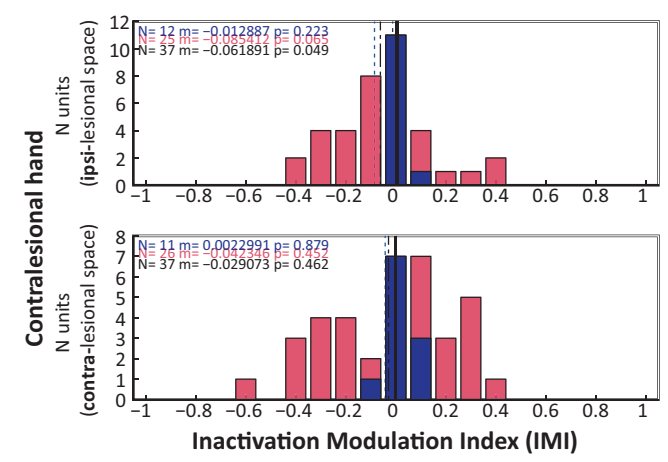

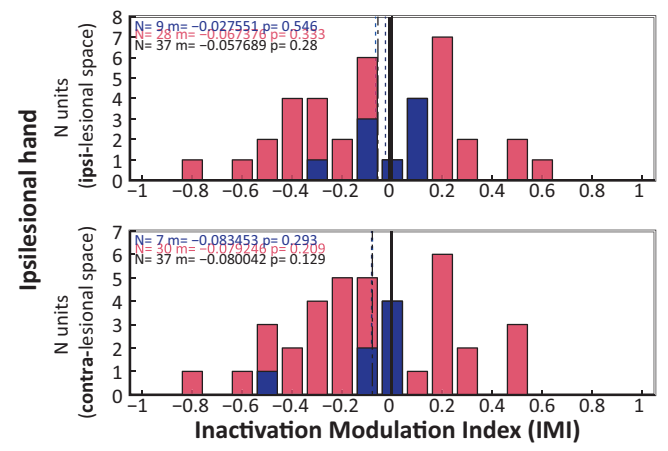

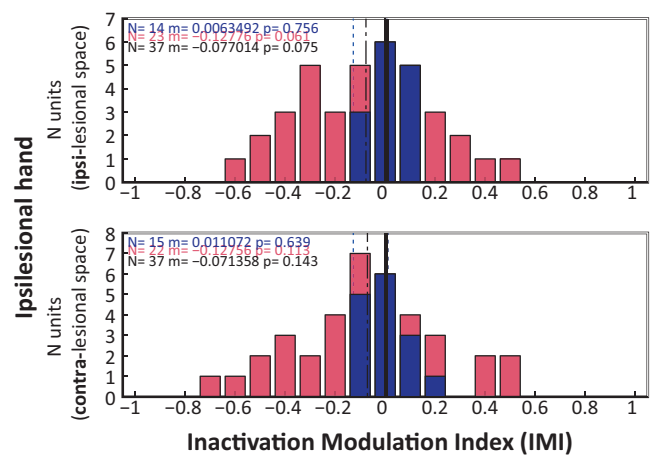

Inactivation Modulation Index (IMI)

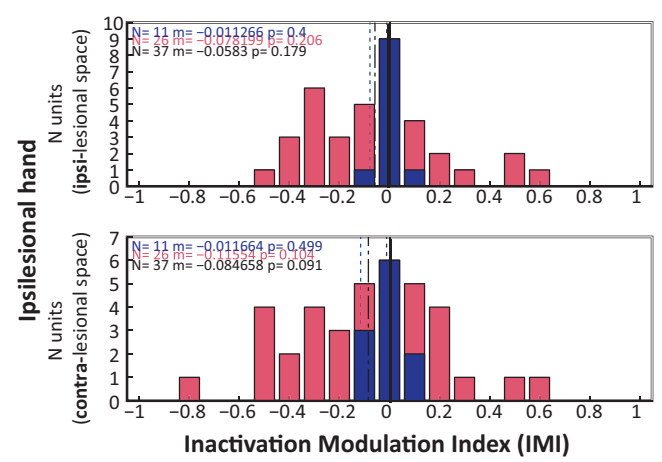

Fig. 3.9 Inactivation modulation index (IMI) for the population of units recorded from the MIP area in right (inactivated) hemisphere. IMI was calculated by subtracting average firing rate in the control trials from the inactivation trials and divided by the sum of two (IMI=(C-I)/(C+I)). IMI was computed for each task period (fixation hold (300-0 ms before cue onset), cue (50-150 ms after cue onset), pre-reach (300-10 ms before reach onset) and each condition (ipsilesional hand, ipsilesional space, contralesional hand, contralesional space) separately. Unpaired ttest was applied between trials in the pre- versus post-injection to get the significant modulation. Dashed vertical lines illustrate means of IMI for the whole population, for the units with significant modulation and for the units with not significant modulation. Bold vertical line just indicate IMI=zero. Negative IMI values indicate decrease of firing rate and positive IMI values means increase of firing rate after inactivation. Red: cells with significant modulations and blue: nonsignificant cells. 
Inactivation modulation index (IMI) for the units in left MIP ( $n=37$ )

a) Fixation hold period

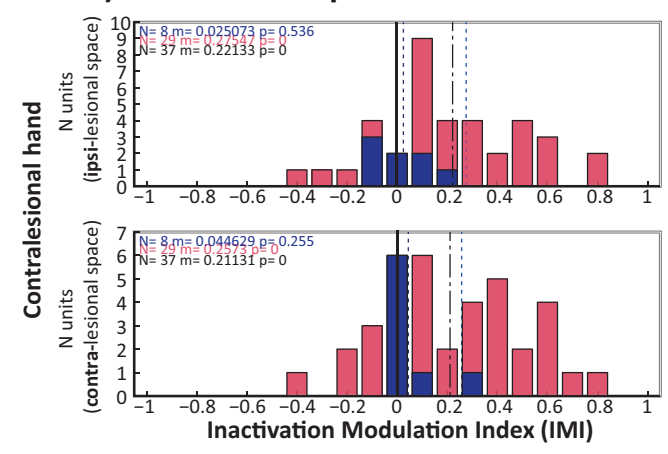

b) Cue period

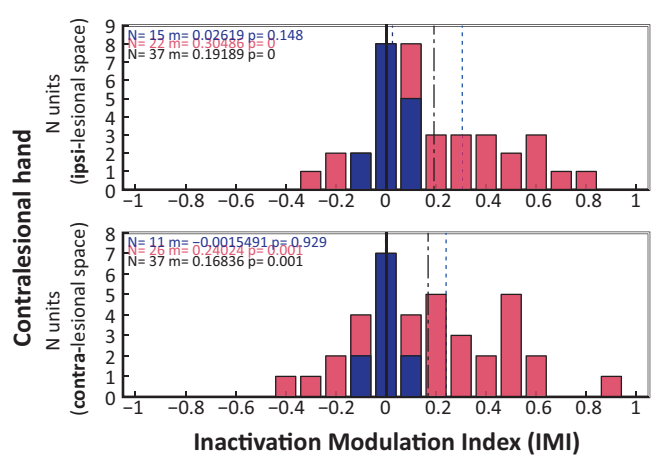

c) pre-reach period

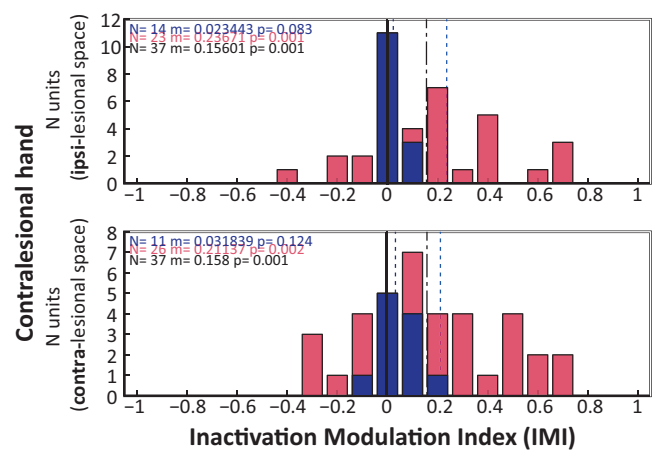

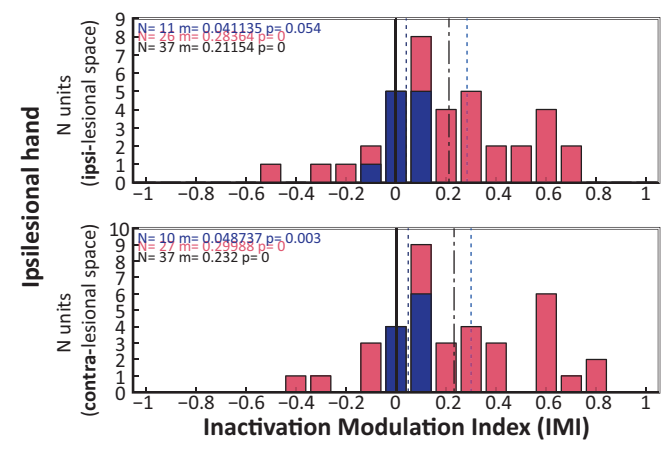
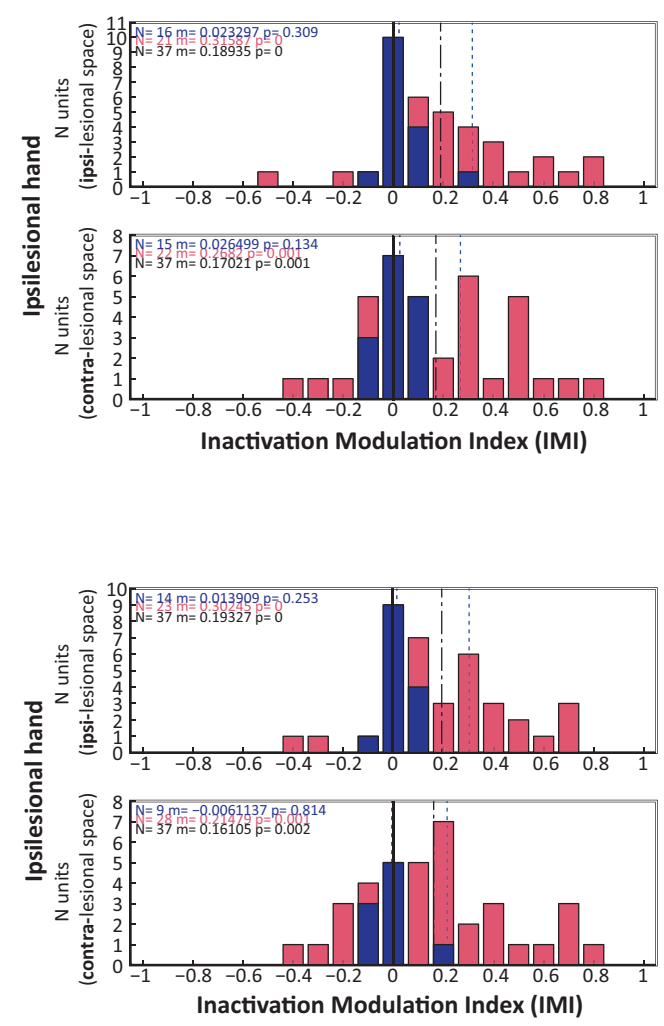

Fig. 3.10 Inactivation modulation index for the population of units recorded from the MIP area in left (intact) hemisphere. Specifications similar to Fig. 3.9. 


\subsubsection{LFP power spectrum changed dramatically after pulvinar inactivation}

In this section we aim to investigate the effects of the unilateral (right) pulvinar pharmacological inactivation on the LFP power spectrum in parietal area MIP while the monkey is performing dissociated delay reaches. The important point is that we normalized the power (z-score; see Methods) for the sake of three main reasons: 1 ) to overcome the $1 /$ f nature of the raw power representation; 2 ) to observe better the condition-specific responses independently for each frequency within each block of trials; 3 ) to be able to see the potential effects of inactivation within each frequency separately.

We normalized the time-frequency spectrograms of each trial using the statistics calculated across all successful trials (including all conditions) in the pre-injection block. Then we averaged these spectrograms across successful trials in each condition (IHIS: ipsi-hand ipsi-space, IHCS:ipsihand contra-space, CHIS:contra-hand ipsi-space, CHCS:contra-hand contra-space). In addition, we calculated for each site the hand-selectivity for space and spatial-selectivity for each hand (ICHd: contra- minus ipsi-hand, ICSd: contra- minus ipsi-space). In total we looked at eight different conditions. We took the right hemisphere (to-be-inactivated) as our reference and define ipsi- and contralesional conditions for each site, in left and right hemisphere, with regard to that.

In the following sections we first show changes in the behavior of the monkey after inactivation. Then we will show the neuronal effects. For both spiking activity and LFP, we first depict the pre-injection (intact state) trials and after that we show how the right pulvinar inactivation affects the neuronal responses (LFP and spiking activity) in each hemisphere. In total we recorded 85 LFP sites (42 from left hemisphere and 43 right hemisphere).

\section{Power spectrum in the intact state}

We asked how the power of the LFP signal distributed across frequencies in each task condition in the intact or normal brain state (pre-injection block).

We looked at the averaged normalized spectrogram (similar to the approach in chapter 2; see Methods section 3.3). In principle, this representation shows how the power in each frequency-bin and time-bin deviates from the mean power across all time bins across all successful trials in the pre-injection block. Fig. 3.11 shows this analysis for the LFP sites recorded in each hemisphere. Here the hand- and space-selectivity panels are after applying FDR corrected p-values: left hemisphere (IHICSd 0.0183; CHICSd 0.0142; ICHdIS 0.0188; ICHdCS 0.0213), right hemisphere (IHICSd 0.0267; CHICSd 0.0200; ICHdIS 0.0333; ICHdCS 0.0273).

In both hemispheres and during the fixation epoch, we observed higher power in the alpha and beta range $(8-30 \mathrm{~Hz})$ with the peak in the low beta range $(13-18 \mathrm{~Hz})$ in all conditions. But there was a weak but significant ipsilesional hand-selectivity. In addition, only in the left hemisphere we saw a significant contralesional hand-selectivity in the delta and theta range. The peak in the beta range was expected since monkey just keeps the central fixation and waits to receive the relevant information for the spatial movement. It has been reported that this kind of idle states increase the beta power (Engel \& Fries, 2010).

While in right hemisphere we saw a strong contralesional cue response in the theta-beta range $(8-30 \mathrm{~Hz})$ which extends to lower gamma, this effect was stronger for ipsilesional cue in the left hemisphere. During the late delay epoch before the go signal, we again saw an increase in the 
power mostly in the lower beta range and weaker in the lower gamma. In right hemisphere, this leads to the ipsilesional space-selectivity for ipsilesional hand and also ipsilesional hand-selectivity for the ipsilesional space. In the left hemisphere, it shows contralesional hand-selectivity for the contralesional space while contralesional space-selectivity for the contralesional hand and opposite for ipsilesional hand. During the movement period the power is low in the beta range and is high in the high gamma and also delta ranges in all conditions. On top of that and only in the right hemisphere, we observed a strong significant contralesional hand-selectivity in the high gamma range and space-selectivity in the delta range.

In addition to instructed trials, monkey performed in a pseudo-random manner in each block also choice trials. Since monkey in many sessions had a strong preference to select a target in the same side as the hand-used (uncrossed conditions), therefore there is lower number of sites for the CHIS and IHCS conditions (crossed conditions). The overall pattern of power distribution is similar to the instructed trials (Fig. 3.12; FDR corrected p-values; left hemisphere: IHICSd 0.0064; CHICSd 0.0069; ICHdIS 0.0129; ICHdCS 0.0108, right hemisphere: IHICSd 0.0152; CHICSd 0.0107; ICHdIS 0.0291; ICHdCS 0.0172).

Here also the peak of the power modulation happens in the lower and higher beta bands and also higher gamma. One difference is that we see no cue space-selectivity since the cue response is similar in all conditions. This is due to the bilateral cue presentation in choice trials. It is worth mentioning that the difference between two hemispheres is minimal and is mostly just stronger beta and gamma modulation in right hemisphere than left but the overall pattern is very similar. 
Average zscored spectrograms in instructed trials in the preinjection block a) across sites in right hemisphere
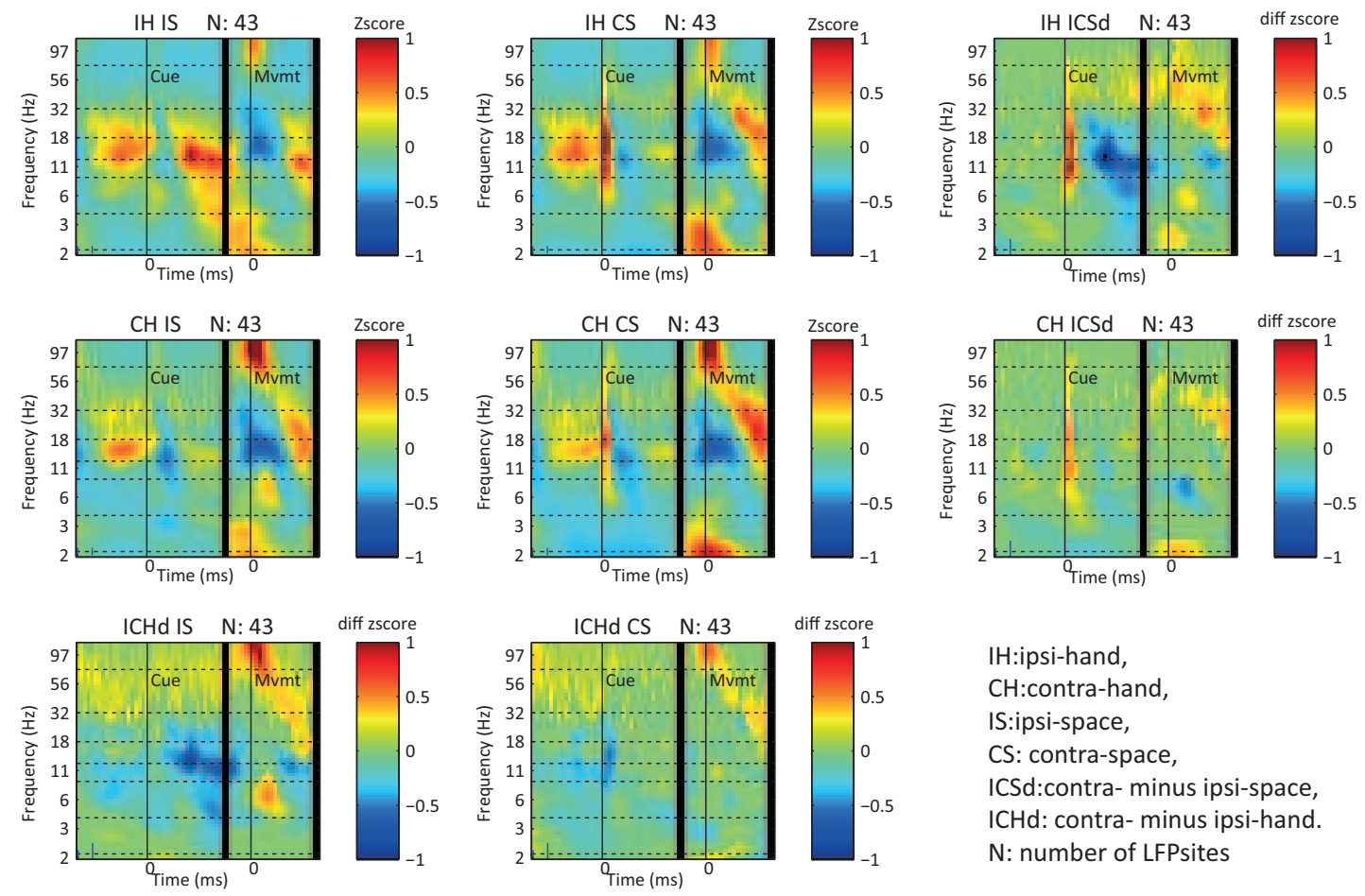

IH:ipsi-hand,

$\mathrm{CH}$ :contra-hand,

IS:ipsi-space,

CS: contra-space,

ICSd:contra- minus ipsi-space, ICHd: contra- minus ipsi-hand. $\mathrm{N}$ : number of LFPsites

b) across sites in left hemisphere
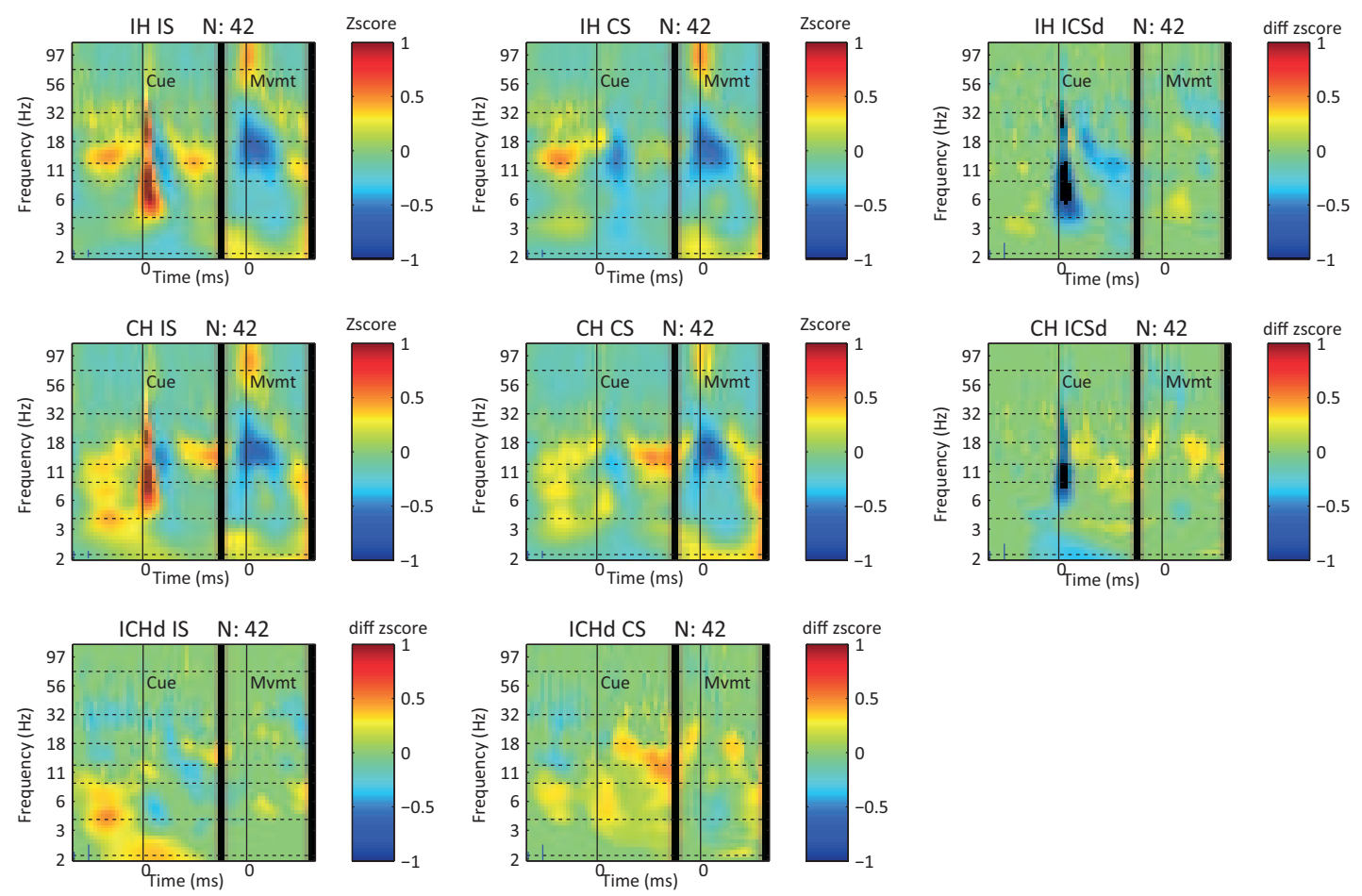

Fig. 3.11 Normalized power spectrum in MIP in each hemisphere in the intact state (preinjection), across sites in left (a) and right (b) hemisphere. Monkey performed the instructed trials of dissociated delay reach task. In (a) and (b), the first $2 * 2$ panels from the top left show separately task conditions. Panels on the first column on the right side and on the last row show the differences across hands or spatial target location. Each panel shows time-frequency representation by averaging the z-scored spectrograms aligned to cue and movement initiation. Ipsi and contra relative to the to-be-inactivated hemisphere (right). 
Average zscored spectrograms in choice trials in the preinjection block a) across sites in right hemisphere
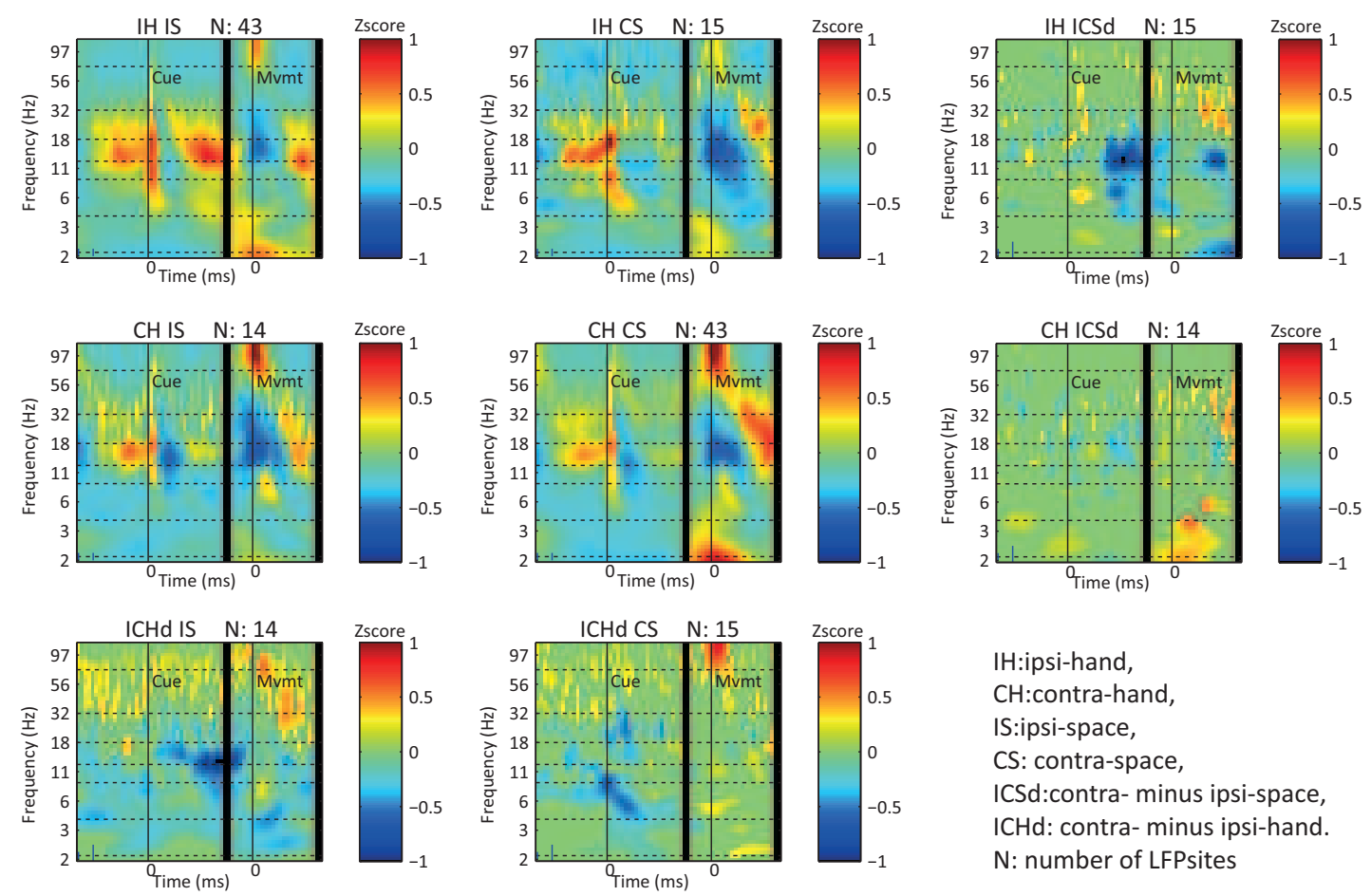

IH:ipsi-hand,

$\mathrm{CH}$ :contra-hand,

IS:ipsi-space,

CS: contra-space,

ICSd:contra- minus ipsi-space,

ICHd: contra- minus ipsi-hand.

$\mathrm{N}$ : number of LFPsites

\section{b) across sites in left hemisphere}
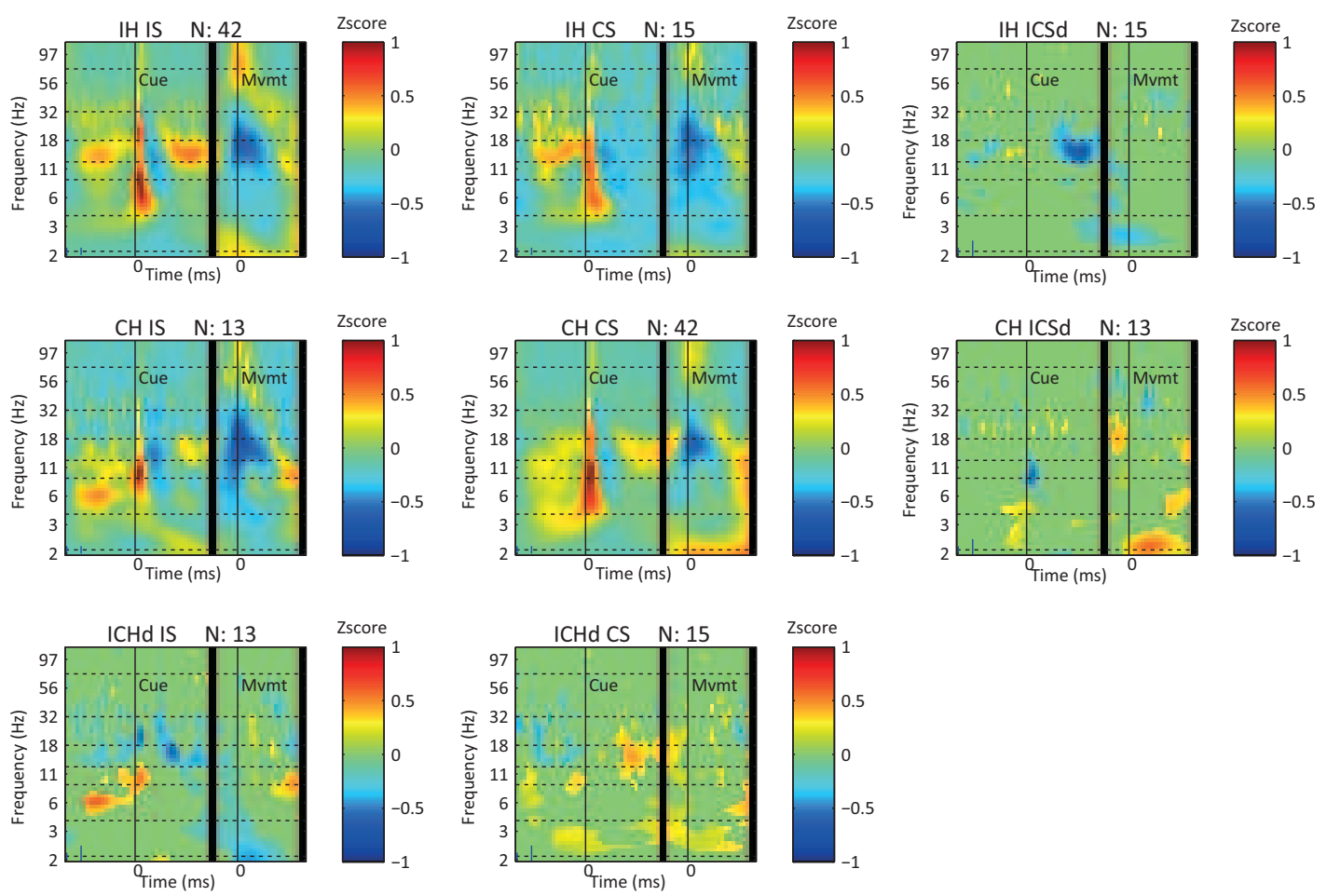

Fig. 3.12 Normalized power spectrum in MIP in each hemisphere similar to Fig. 3.11 but for choice trials. 


\section{Changes of MIP power spectrum within $\sim 1$ hour after pulvinar inactivation}

First we aim to see how the power of the LFP signal in the MIP area in each hemisphere changed after inactivating only pulvinar in the right hemisphere. Fig. 3.13 depicts the effects in the first block of trials after inactivation in the left (a) and right hemisphere (b). Here we show only the effects in the main four task conditions. Each panel is a time-frequency representation which is the difference between averaged z-scored spectrograms across sites in the post- relative to average across sites the pre-injection block. Only time-frequency bins which are significant (see Methods) are shown and the white color shows not-significant differences.

In right hemisphere and regardless of the task condition, we observed a significant increase of power in the delta, theta and alpha bands for all epoch before the movement and a weak (not significant) increase also in gamma band. Interestingly, while for the ipsilesional hand reaches, beta power slightly decreased (not significant; $\mathrm{p}>0.05$ ) during fixation hold, cue and delay period, contralesional hand reaches showed an increase in beta range.

But in the intact left hemisphere, we observed an opposite effect. Power in the lower frequency bands (delta, theta, alpha) decreased slightly (mostly not significant) but in the beta and gamma range significantly increased $(\mathrm{p}<0.05)$. This effect remained similar for all four task conditions. Interestingly during fxh period and only for ipsilesional hand power significantly increased in the whole beta range.

\section{Effect-size 1 hour after unilateral pulvinar inactivation (Inst trials)}

a) across sites in left hemi (intact)
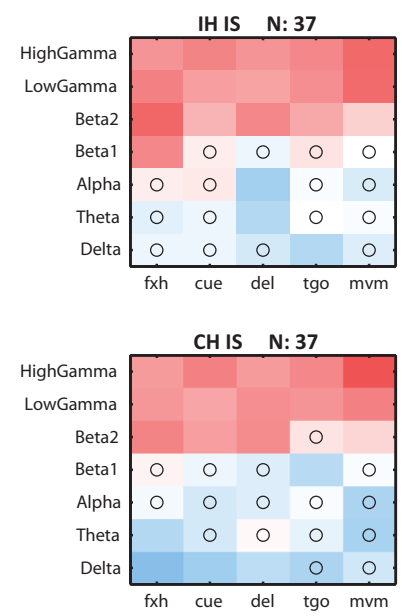

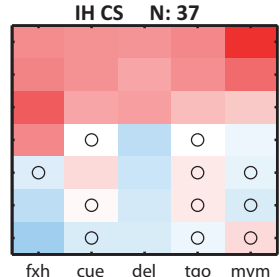

CH CS N: 37

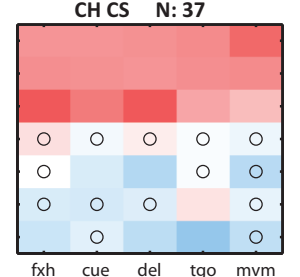

b) across sites in right hemi (inactivated)
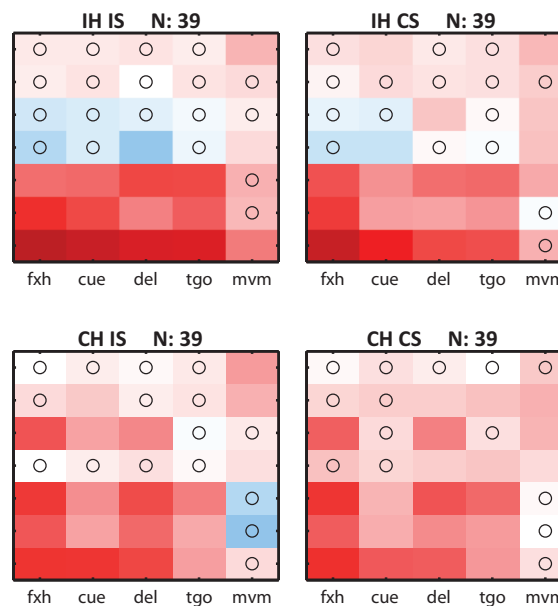

Fig. 3.13 Effect size of inactivation on the first block of trials within $\sim 1$ hour after injection) across sites in the left hemisphere (a) as well as in the right hemisphere (b). Effect size defined as the changes in the normalized power after the injection. Each panel shows one task condition. Each pixel shows the effect size in one epoch and for one frequency-band averaged across sites. Small circles depict non-significant effects (no changes after injection) using paired ttest. Ipsi/Contra relative to the inactivated hemisphere (right). Epochs: 'fxh': 0-500 ms before the cue; 'cue': 0-300 ms after the cue onset; 'del': 600-1000 ms after the cue onset; 'tgo': 0-400 ms before the movement onset; 'mvm': 0-400 ms after the movement onset

Generally, the effects of pharmacological inactivation increased with time. In the next section we will explain in more detail the significance of effects on the population level as well as individual LFP sites for the second block of trials after the injection. 


\section{Changes of MIP power spectrum within $\sim 2$-3hours after pulvinar inactivation}

Following we will show that generally the pattern of effects in the second block of trials (about 2-3 hours after injection) are similar to the first block but the effects are stronger. We first investigate the changes in time-frequency maps in each hemisphere separately. In this analysis each panel of spectrogram shows the bins which has a significant change after inactivation on the population level (white bins are non-significant bins; p-value corrected by FDR method).

In the right hemisphere (Fig. 3.14; FDR correct p-values: IHIS 0.0264; IHCS 0.0268; CHIS 0.0272; CHCS 0.0304; IHICSd 0.0149; CHICSd 0.0145; ICHdIS 0.0219; ICHdCS 0.0207), there was a significant increase in the power in lower frequencies (delta, theta, alpha) through whole trial except the movement period. In all four conditions during movement execution power in the theta and alpha bands did not change. Gamma power especially high gamma showed almost no significant changes after the inactivation but the interesting effect happened in the beta range. Higher beta power increased in all epochs except movement for the contralesional hand but decreased for the ipsilesional hand. In addition, while lower beta did not change for contralesional hand conditions and decreased significantly for ipsilesional hand conditions.

According to the selectivity panels, the contralesional hand-selectivity increased significantly in the beta range. We also saw a significant decrease in the hand- and space-selectivity in the delta band only between cue and delay periods. Additionally, contralesional space-selectivity increased in the late delay period only for the ipsilesional hand.

Fig. 3.15 illustrates the effects in the intact left hemisphere. Similar to results in the first inactivation block of trials, we have a strong opposite effect in the left hemisphere (FDR corrected p-values: IHIS 0.0259; IHCS 0.0241; CHIS 0.0262; CHCS 0.0283; IHICSd 0.0058; CHICSd 0.0076; ICHdIS 0.0079; ICHdCS 0.0074). The power in higher beta and gamma range increased significantly after inactivation for all the epochs except movement period in beta band. This increase was slightly stronger in the beta during the fixation hold for the ipsilesional hand. In contrast, we observed a significant decrease in the alpha and lower beta range during the delay period stronger for the contralesional hand. In contrast to right hemisphere, power in the lower frequency bands (delta, theta and alpha) did not dramatically change after inactivation. One significant change was that power in the delta range decreases around the movement before and after movement execution only for the contralesional hand. 
a) Changes in spectrograms after inactivation (across sites in right hemi)
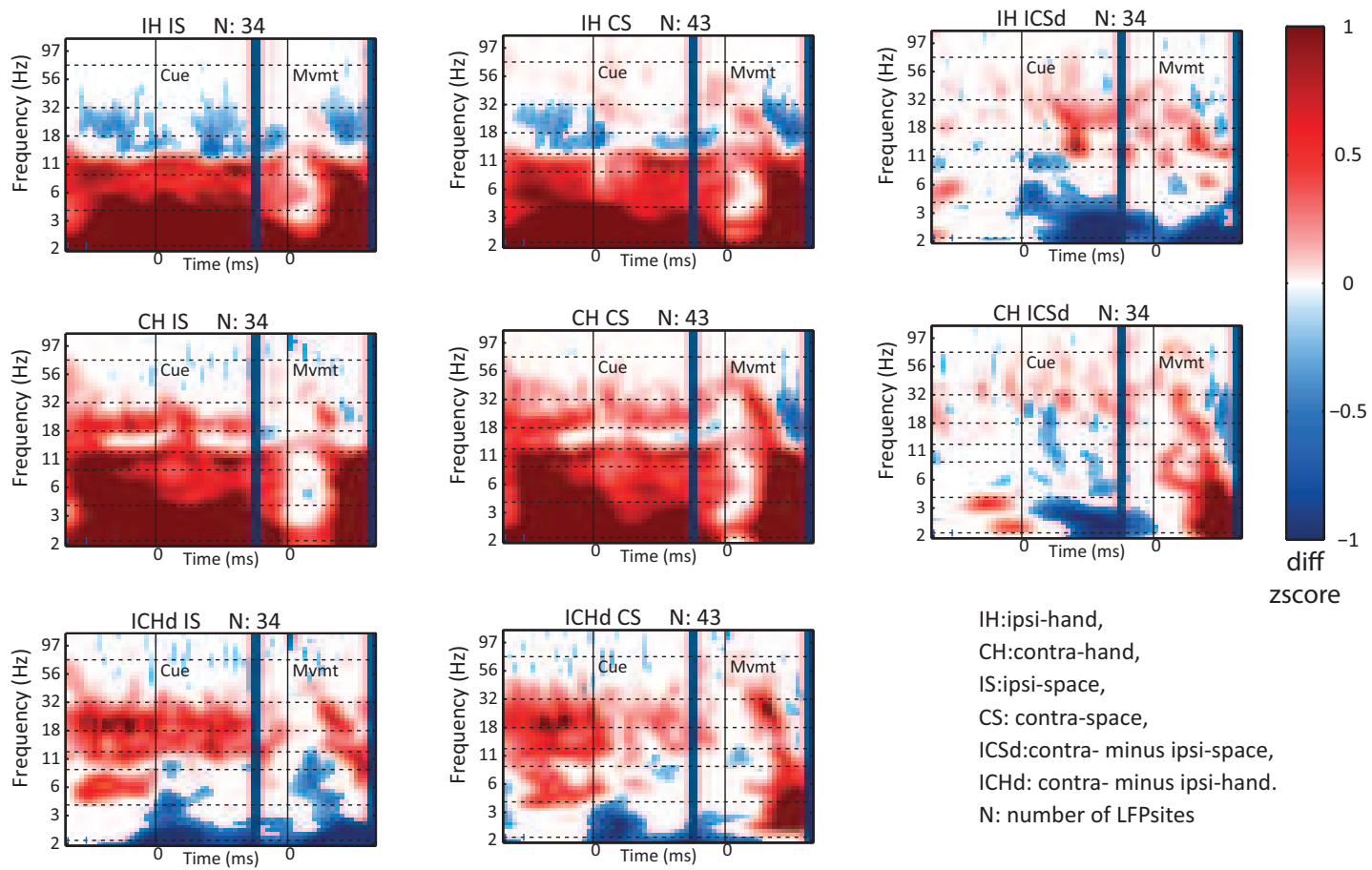

IH:ipsi-hand,

$\mathrm{CH}$ :contra-hand,

IS:ipsi-space,

CS: contra-space,

ICSd:contra- minus ipsi-space,

$\mathrm{ICHd}$ : contra- minus ipsi-hand.

$\mathrm{N}$ : number of LFPsites

Fig. 3.14 Significant changes in the averaged normalized power after inactivation on the second block of instructed trials ( 2-3 hour after injection) across sites in the right hemisphere. a) Significant changes in the spectrograms. For each time-frequency bin, if there was a significant (ttest with a FDR-corrected critical p-value) difference between pre- and post-injection block the difference (effect size) is shown. 
a) Changes in spectrograms after inactivation (across sites in left hemi)
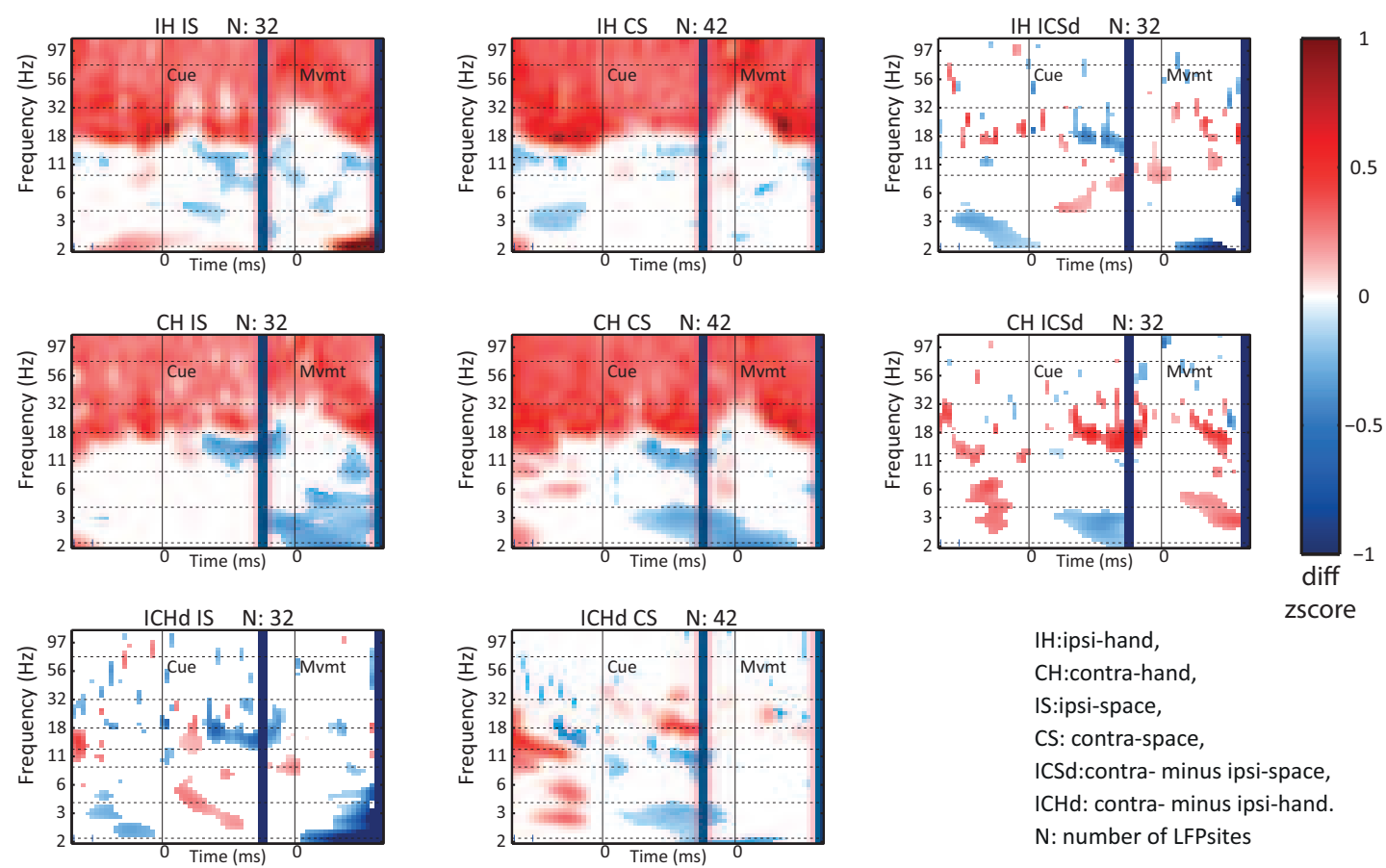

IH:ipsi-hand,

$\mathrm{CH}$ :contra-hand,

IS:ipsi-space,

CS: contra-space,

ICSd:contra- minus ipsi-space, ICHd: contra- minus ipsi-hand. $\mathrm{N}$ : number of LFPsites

Fig. 3.15 similar to Fig. 3.14 but for the sites in the left hemisphere.

In this hemisphere, though, we did not observe clear changes in the selectivity maps. One may notice that the contralesional space-selectivity increased for the contralesional hand and the contralesional hand-selectivity decreased for the ipsilesional targets in the beta band during late delay period.

Fig. 3.14(b) and Fig. 3.15(b) are showing the same result as (a) in the same figures but for each frequency band separately. This helps us to identify the time-course of changes of power in each of seven defined frequency bands. This replicates the effects we described on the changes in spectrograms. As the most apparent effect was the increase of power in lower bands (delta, theta and alpha) in the right (inactivated) hemisphere and also increase of the higher bands (high-beta and gamma) in the left hemisphere, but interestingly the beta band power changed differently. Alpha and lower beta decreased in left hemisphere especially stronger for the contralesional hand but in the right hemisphere low and high beta increased for the contralesional hand but decreased for the ipsilesional hand especially during fixation hold and delay epochs.

We then tested the significance of changes (effect-size) in each frequency band and epoch as explained for the Fig. 3.13. Fig. 3.16 shows this analysis for the second block of trials after inactivation, (a) for the sites in the right and (b) for the sites in the left hemisphere. In addition, we also show the effects on the hand- and space-selectivity maps.

This analysis confirms the effects that we explained on the spectrograms. In the right hemisphere, normalized power in beta and to some extend alpha band shows increase in the contralesional handselectivity. In this hemisphere, although gamma band does not significantly change in almost all 
conditions but there is a significant decrease in the contralesional hand-selectivity. This effect is almost constant through the trial and is not epoch specific.

One of the most apparent effects was the increase of beta band contralesional-hand selectivity which was stronger during fixation, cue and delay. This effect nicely goes along our hypothesis. In the cue period we see a decrease in the contralesional-space selectivity in the below beta frequency bands which is stronger for the contralesional hand condition. That means contralesional-space selectivity decreased after inactivation stronger for contralesional hand. In addition, during del, go and mvm epochs only in the beta and low gamma bands contralesional space-selectivity increases. This effect is stronger with the contralesional hand.

In the left hemisphere, we observed a significant increase in the beta band contralesional spaceselectivity during del and tgo epochs only for the contralesional hand. Also we observed a significant decrease in the contralesional hand-selectivity during fxh epoch for the contralesional targets and opposite in the lower (delta-theta-alpha) range. But during del and tgo epochs, beta band showed a decrease of the contralesional hand-selectivity for the ipsilesional targets.

As described in the Methods, monkey performed choice as well as instructed trials in a block. So far we talked only about the neuronal effects on the instructed trials. We also asked whether those results hold for the choice trials (Fig. 3.17 (a) right (b) left hemisphere). In choice trials, the general effect in each condition is similar to what we see for the instructed trials therefore we just show the selectivity maps. One of the main differences to instructed trials is that in the delta and theta bands we observe a significant decrease in the contralesional hand-selectivity for the contralesional space. This was much weaker in the instructed trials. Opposite to the instructed trials, in the cue epoch there is no significant (weak increase) contralesional space-selectivity for contralesional hand.

In both hemispheres and during the fxh period beta band shows significant decrease in the contralesional space-selectivity with the contralesional hand. This effect is not unexpected because in this period monkey does not have any spatial information (and also any choice vs instructed information) and therefore there should be no space-selectivity and no difference between choice and instructed. 


\section{a) Effect-size of unilateral pulvinar inactivation (right MIP)}
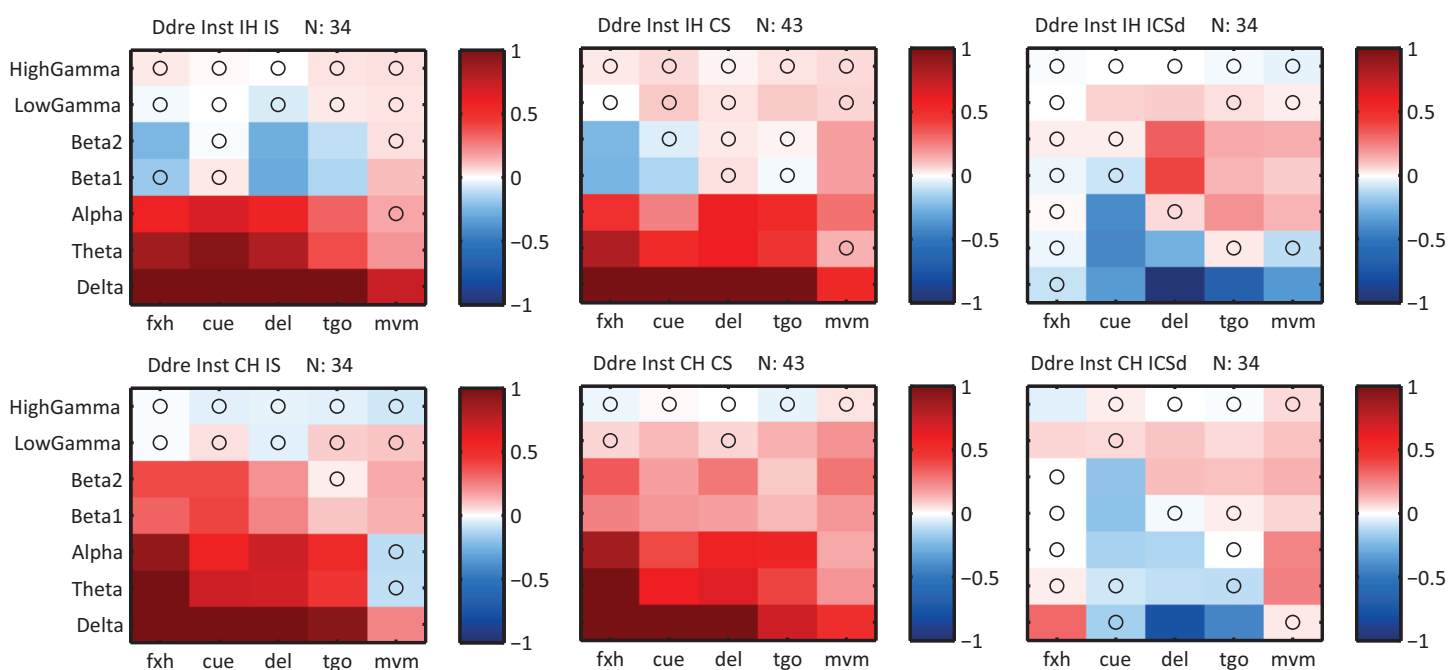

Ddre Inst CH ICSd N: 34
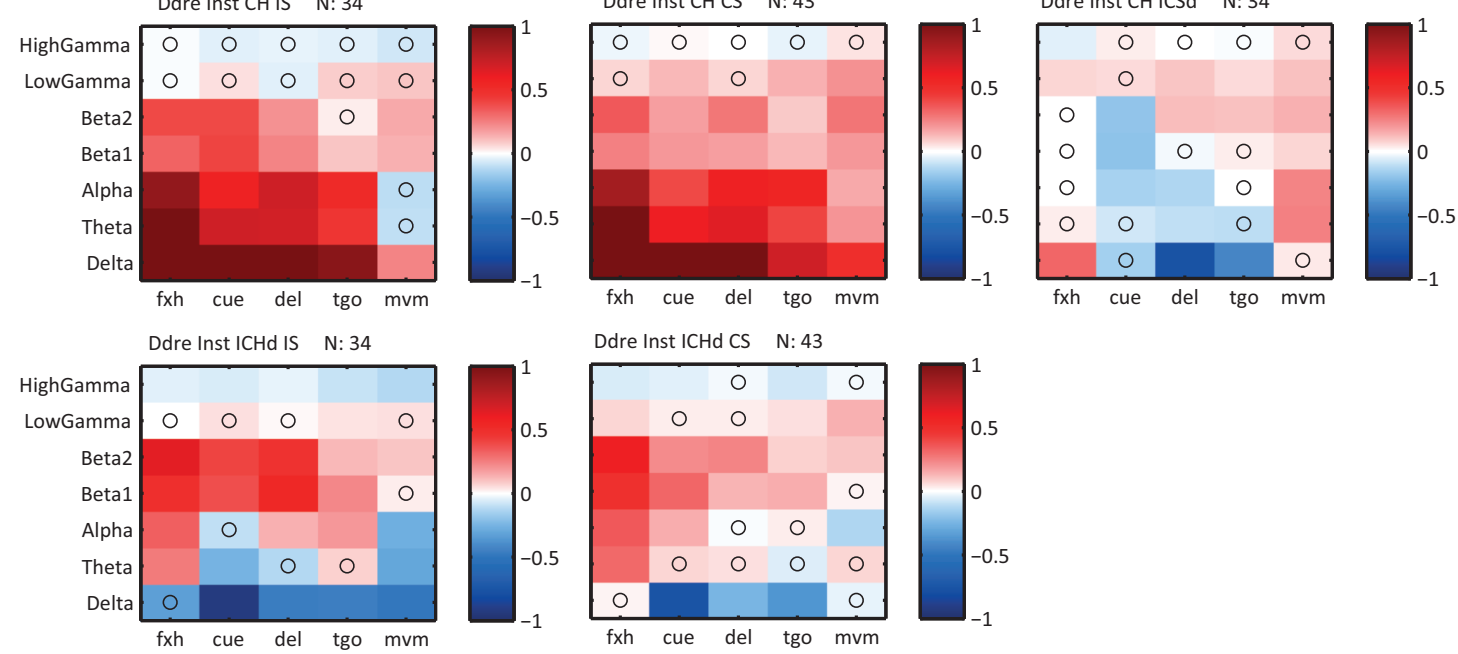

\section{b) Effect-size of unilateral pulvinar inactivation (left MIP)}
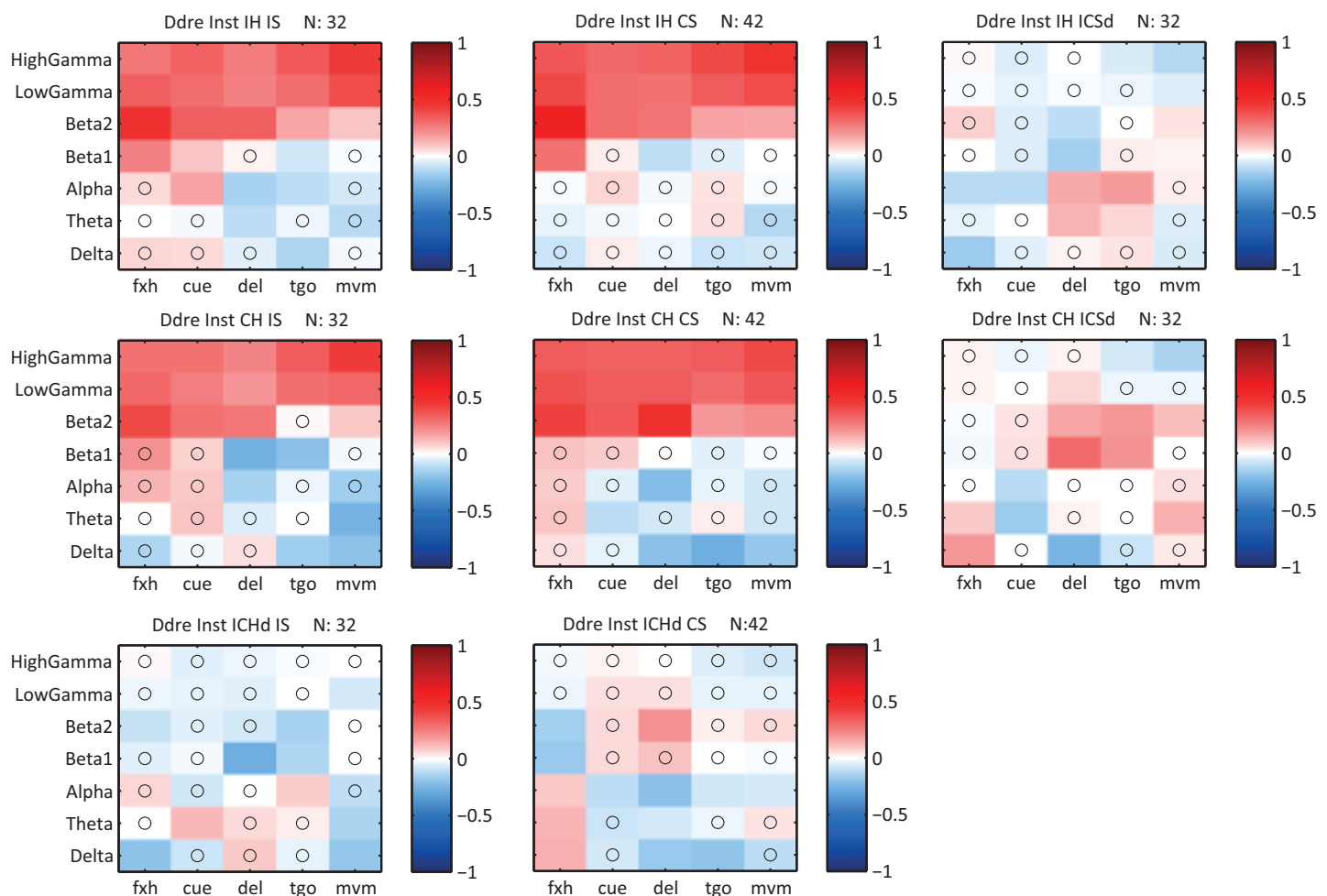

Fig. 3.16 Effect size of inactivation on the second block of instructed trials ( $~ 2-3$ hour after injection) across sites in the right (a) and left (b) hemisphere. General format the same as Fig. 3.13. In addition to single conditions, we show the effects on hand- and space-selectivity maps: ICSd(contra-space minus ipsi-space) shows spatial-selectivity and ICSd(contra-hand minus ipsi-hand) shows hand-selectivity. 


\section{a) Effect-size in choice trials (right MIP)}
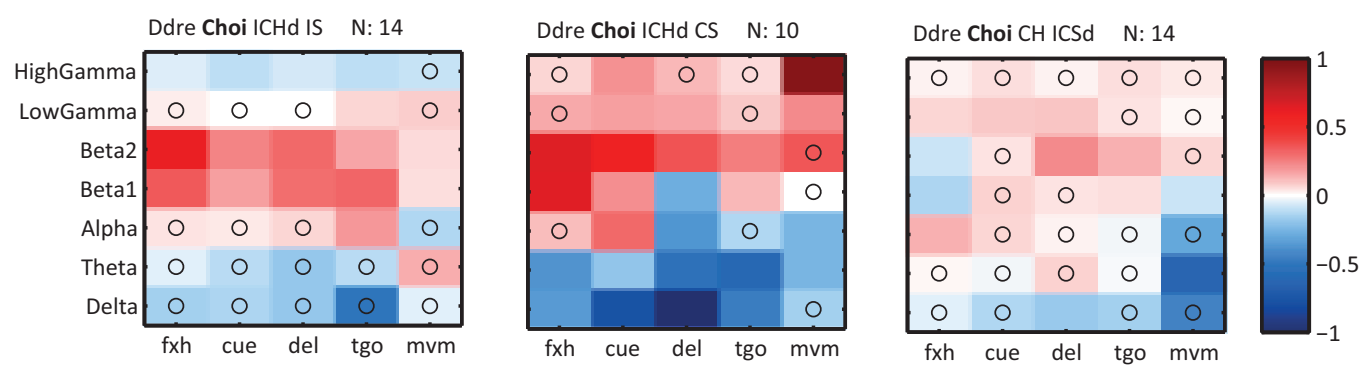

\section{b) Effect-size in choice trials (left MIP)}
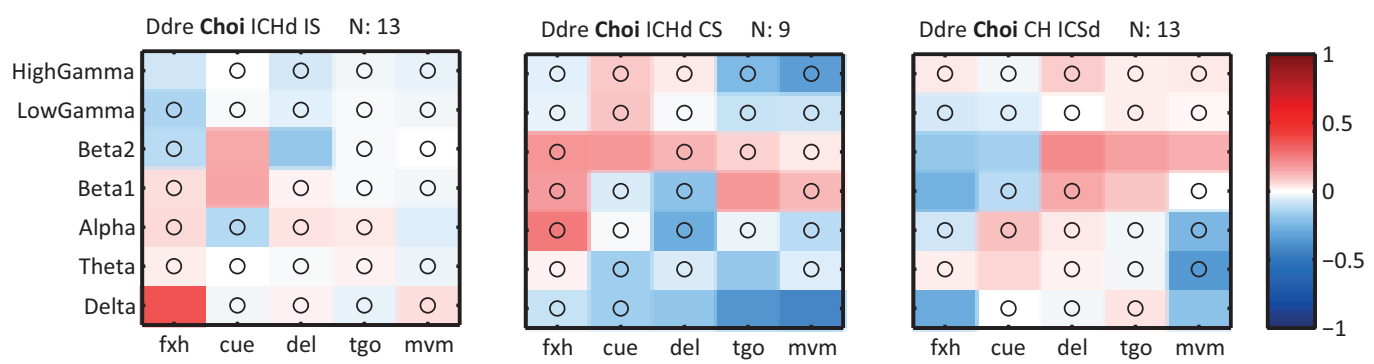

Fig. 3.17 Effect size of inactivation on the second block of choice trials in the hand- and space-selectivity maps (Similar to Fig. 3.16), (a) sites in right hemisphere (b) sites in left hemisphere. The space selectivity condition with ipsilesional hand (IHICSd) is not included since there was not enough trials per LFP site to compute the difference between ipsi- and contralesional target selections and this was due to the monkey being biased to select mostly targets congruent to the acting hand-space.

In addition to the overall averaged effect size on the population, we asked how the effect size distributed across individual LFP sites. Fig. 3.18, Fig. 3.19 and Fig. 3.20 show these scatter plots for the effects in different frequency bands in the right (inactivated) and Fig. 3.21, Fig. 3.22 and Fig. 3.23 show the effects in the left (intact) hemisphere. The statistics (actual p-values) is added only for significant epochs in each condition.

In general, the variability of effect size across individual LFP sites was higher in the lower frequencies including delta, theta, alpha and lower beta than in higher frequencies in which the effects seem to be more consistent. In right hemisphere and for the low frequency bands almost all LFP sites showed increase their power after inactivation in the four main task conditions. The explained effect in the beta band is less consistent across sites but still highly significant. As we see in the average effect size plots, in the right hemisphere gamma band, most of the sites stay along the diagonal and show not a significant effect.

Similarly, in the left hemisphere frequency band which had a significant effect (higher beta and gamma bands) had a consistent effect across the population of sites. For the nonsignificant bands we saw generally a cloud of values around zero which suggests that the modulation of power across epochs is small in the first place and on top of that there is not much effect of pulvinar inactivation on lower frequency bands. 


\section{Low frequency- and epoch-specific effects on individual sites in MIP right hemisphere}

\section{Delta [2 4) Hz}
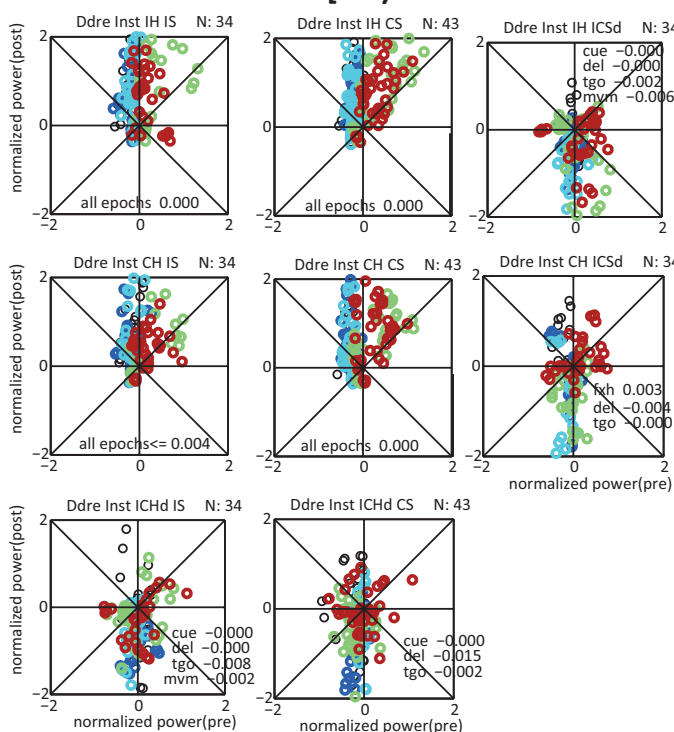

Alpha $[8$ 11) $\mathrm{Hz}$
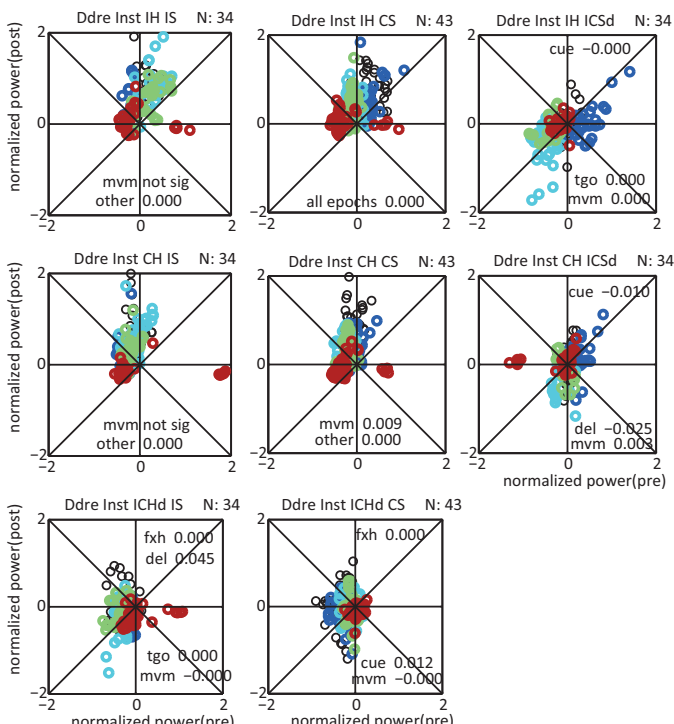

Theta [4 8) $\mathrm{Hz}$
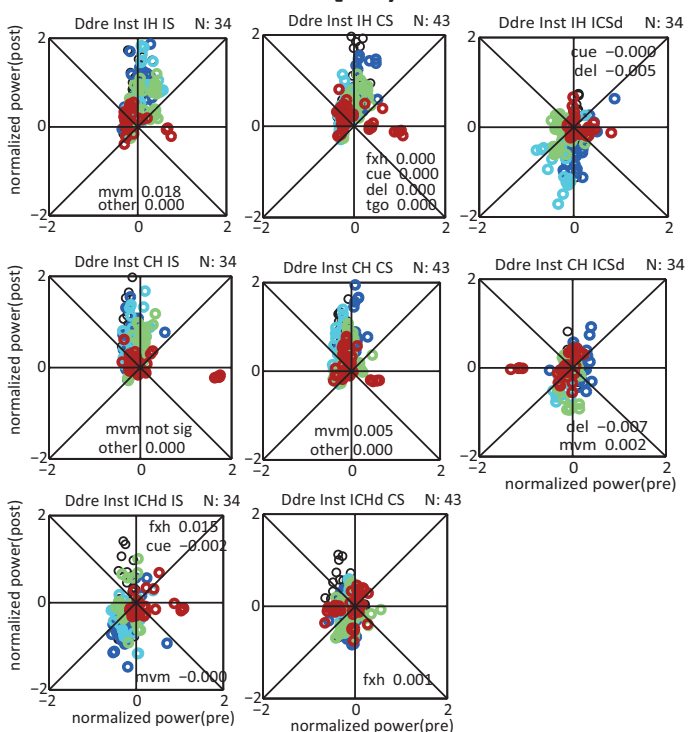

fxh (500-0 ms before cue)

cue (0-300 after cue)

del (600-1000 ms after cue)

tgo (400-0 ms before

movement onset)

mvm (0-400 ms after

movement onset).

Fig. 3.18 Averaged normalized power (across instructed trials) in low frequency bands and each epoch per LFP site in right (inactivated) hemisphere in the pre- versus post-injection block. Each panel corresponds to one condition in one frequency band. For each frequency band we plot 8 conditions as before (four main conditions and four hand- or space-selectivity conditions). Each circle represents one LFP site. Colors represents task epochs. The significant p-value (paired ttest across sites) is mentioned in each panel only if the comparison was significant. Contra and ipsi are relative to the inactivated hemisphere. 
Beta frequency- and epoch-specific effects on individual sites in MIP right hemisphere

Beta1 [11 18) Hz
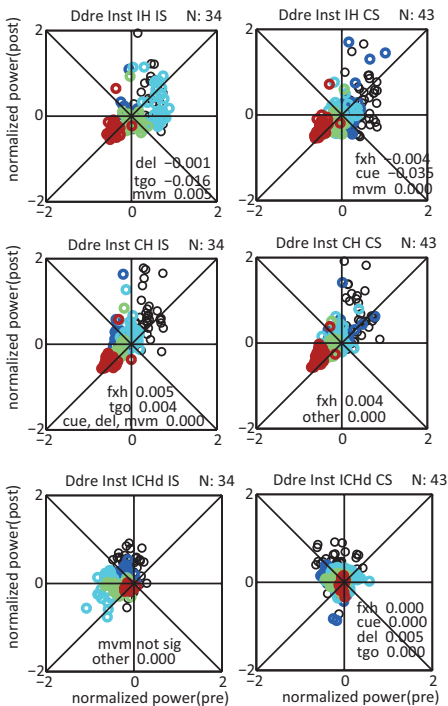
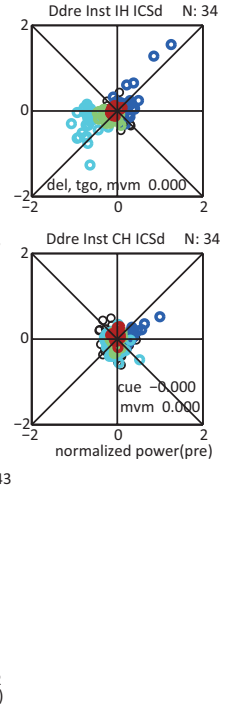

Fig. 3.19 Averaged normalized power (across instructed trials) in beta frequency and each epoch per LFP site in right (inactivated) hemisphere in the pre- versus post-injection block. Details similar to Fig. 3.18.

\section{Gamma frequency- and epoch-specific effects on individual sites in MIP right hemisphere Low Gamma [30 70) Hz}
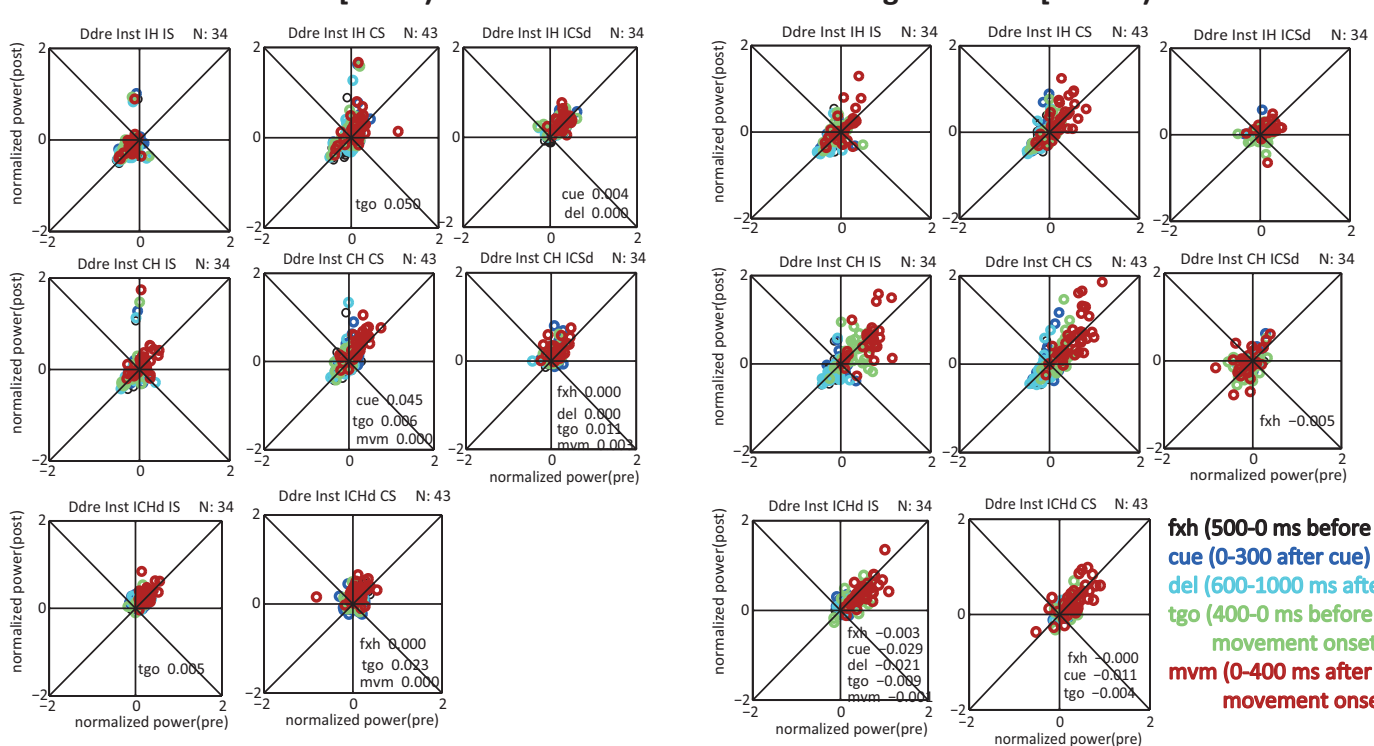

fxh (500-0 $\mathrm{ms}$ before cue) cue (0-300 after cue) del (600-1000 ms after cue) tgo (400-0 ms before movement onset) mvm (0-400 ms after movement onset).

Fig. 3.20 Averaged normalized power (across instructed trials) in gamma frequency and each epoch per LFP site in right (inactivated) hemisphere in the pre- versus post-injection block. Details similar to Fig. 3.18. 


\section{Low frequency- and epoch-specific effects on individual sites in MIP left hemisphere}

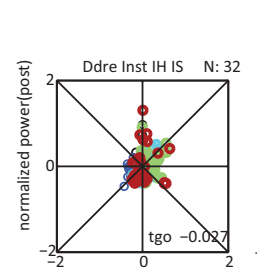

\section{Delta [2 4) $\mathrm{Hz}$}
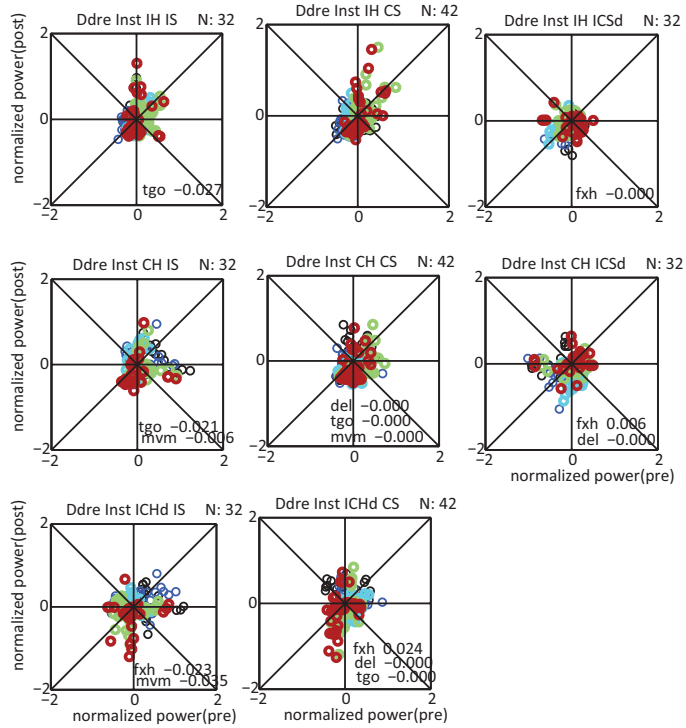

Alpha $[8$ 11) $\mathrm{Hz}$
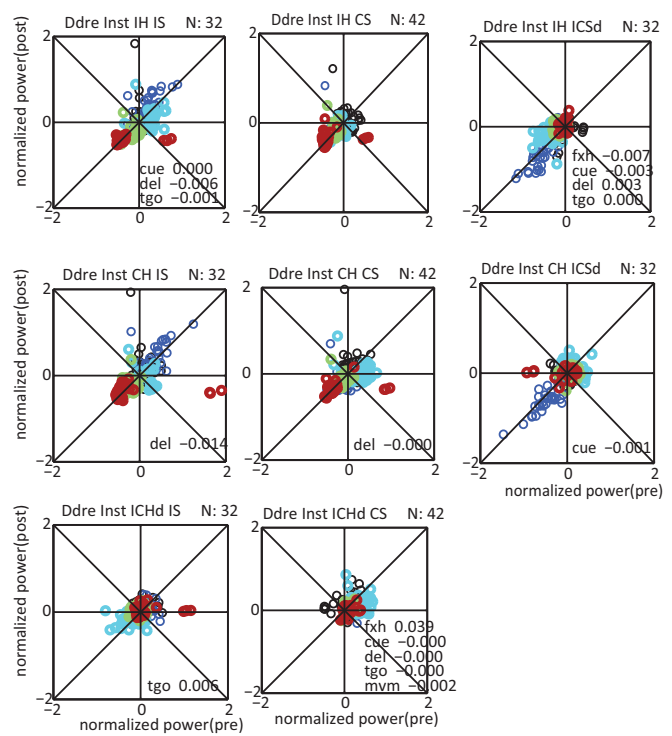

Theta [4 8) $\mathrm{Hz}$
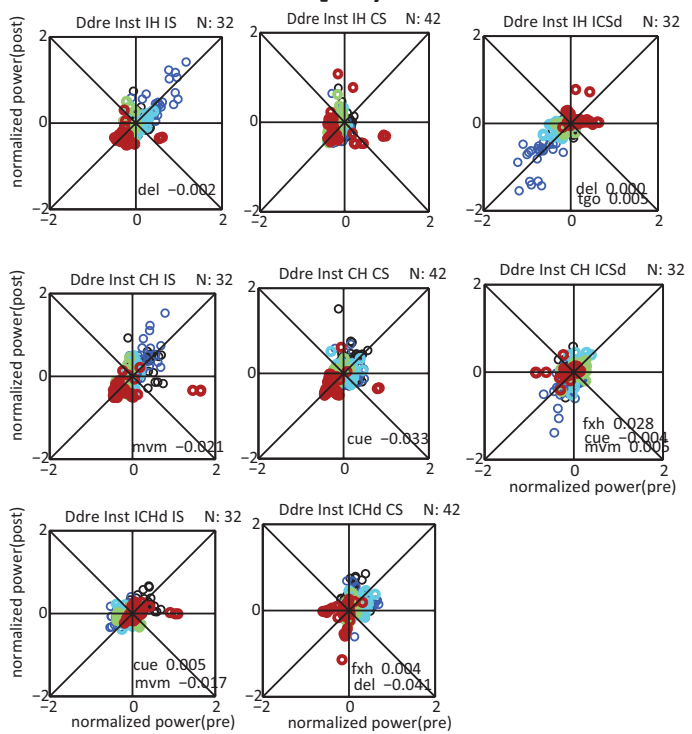

fxh (500-0 ms before cue)

cue (0-300 after cue)

del (600-1000 ms after cue)

tgo (400-0 ms before

movement onset)

mvm (0-400 ms after

movement onset).

Fig. 3.21 Averaged normalized power (across instructed trials) in low frequency bands and each epoch per LFP site in left (intact) hemisphere in the pre- versus post-injection block. Details similar to Fig. 3.18. 
Beta frequency- and epoch-specific effects on individual sites in MIP left hemisphere Beta1 [11 18) $\mathrm{Hz}$

Beta2 [18 30) Hz
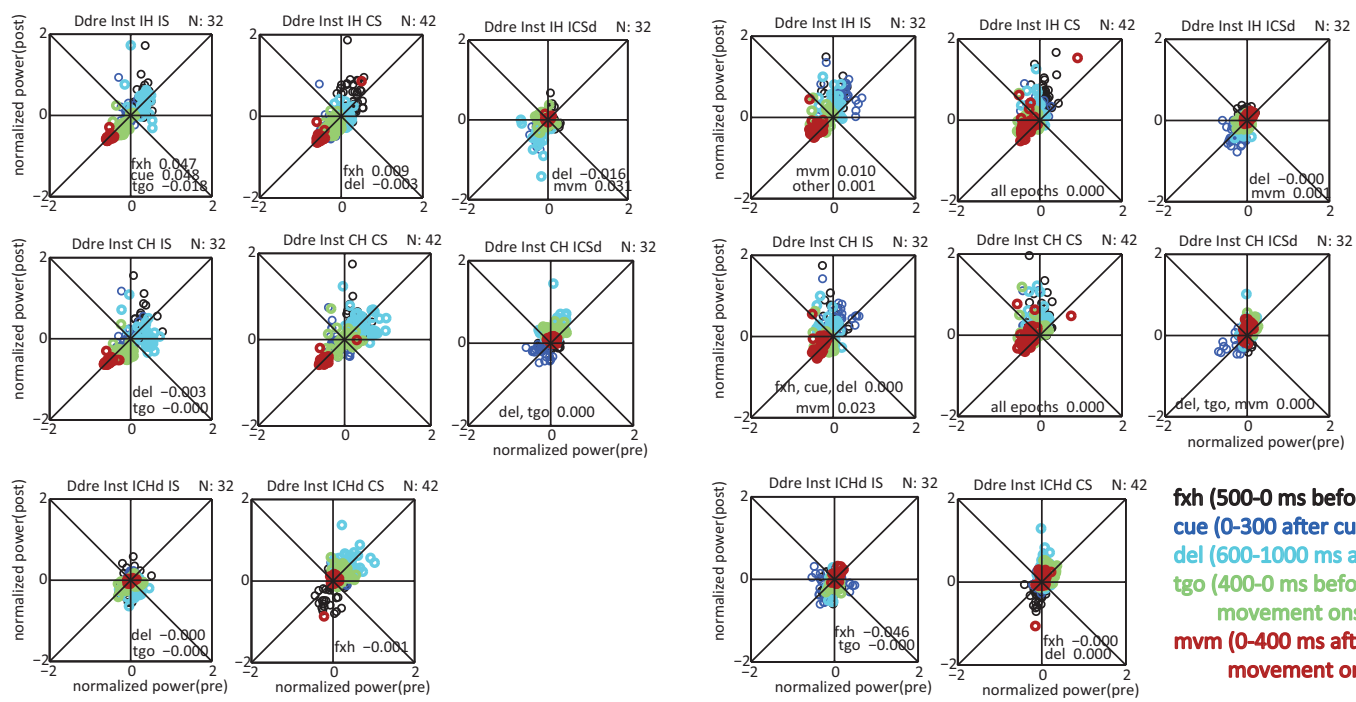

fxh (500-0 ms before cue) cue (0-300 after cue) del (600-1000 ms after cue) tgo (400-0 $\mathrm{ms}$ before movement onset) mvm (0-400 $\mathrm{ms}$ after movement onset).

Fig. 3.22 Averaged normalized power (across instructed trials) in beta frequency and each epoch per LFP site in left (intact) hemisphere in the pre- versus post-injection block. Details similar to Fig. 3.18.

\section{Gamma frequency- and epoch-specific effects on individual sites in MIP left hemisphere \\ Low Gamma [30 70) Hz \\ High Gamma [70 120)Hz}
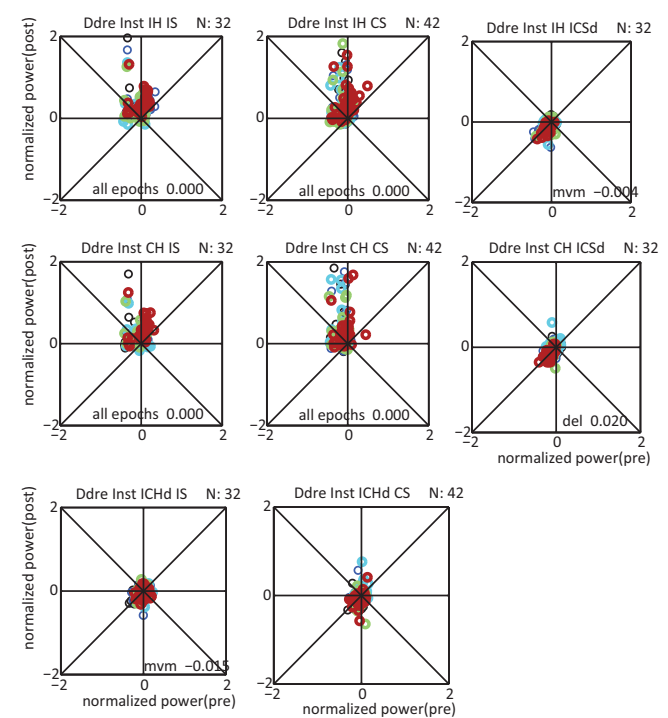
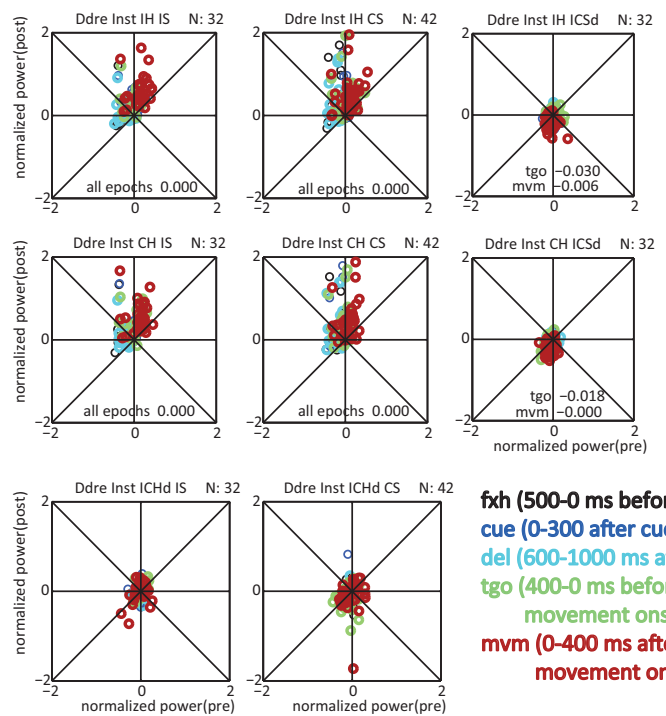

fxh (500-0 ms before cue) cue (0-300 after cue) del (600-1000 ms after cue) tgo (400-0 ms before movement onset) mvm (0-400 ms after movement onset).

Fig. 3.23 Averaged normalized power (across instructed trials) in gamma frequency and each epoch per LFP site in left (intact) hemisphere in the pre- versus post-injection block. Details similar to Fig. 3.18. 


\subsubsection{Unilateral pulvinar inactivation alters single/multi-unit activities in area LIP}

Next we asked to which extend the neuronal effects are area-specific and also interestingly taskspecific. To approach this question, we recorded neuronal signals from area lateral intraparietal (LIP) simultaneously from both hemispheres while the monkey performed the dissociated delay saccade task (Ddsa). We decided to let the monkey do this task while LIP recording because from a numerous previous studies it is already known that area LIP is more responding to eye- than arm-movements (for review see (Battaglia-Mayer et al., 2016)). Since tasks always were performed in a block-wise manner, we assume that monkey knew from the first a few trials which task he will perform. The beginning of Ddsa task is the same as Ddre task (fixation acquisition and fixation hold periods). Until spatial cue presentation monkey had to perform a reach to the middle of the screen and keep his hand there for a few hundred milliseconds while also holding eye-fixation close to his hand position. Therefore, the only difference between performing these two task during fixation acquisition and fixation hold is that monkey was aware that he is going to do the rest of the job with an eye-movement or a hand-movement. In total, we recorded 50 units ( 23 from left and 27 from right hemisphere) and also 39 LFP sites (19 from left and 20 from right hemisphere) within 4 experimental sessions.

Fig. 3.24 illustrates two examples LIP units from right hemisphere in the same session but recorded from separate channels with $2.3 \mathrm{~mm}$ distance in depth that pulvinar inactivation caused different effects on them. In the first unit (Fig. 3.24(a)), pulvinar inactivation increased the firing rate only for the saccade targets in the ipsilesional space (regardless of the fixated hand) mostly after cue presentation until the saccade movement offset. In the second unit (Fig. 3.24(b)), pulvinar inactivation decreased the firing rates mostly for the saccade targets in the contralesional space for both hands but stronger when the contralesional hand was fixated.

Similar to the analysis on the MIP units, we calculated the inactivation modulation index (see 3.4.2) for the units in the LIP of each hemisphere (Fig. 3.25 and Fig. 3.26). This analysis reveals that on the population level there is no effect of the population since many units show increased and many showed decreased of firing rates (besides units that showed no effect). 
a) Unit “Lin_20171023_10” right LIP (Dissociated saccade task)
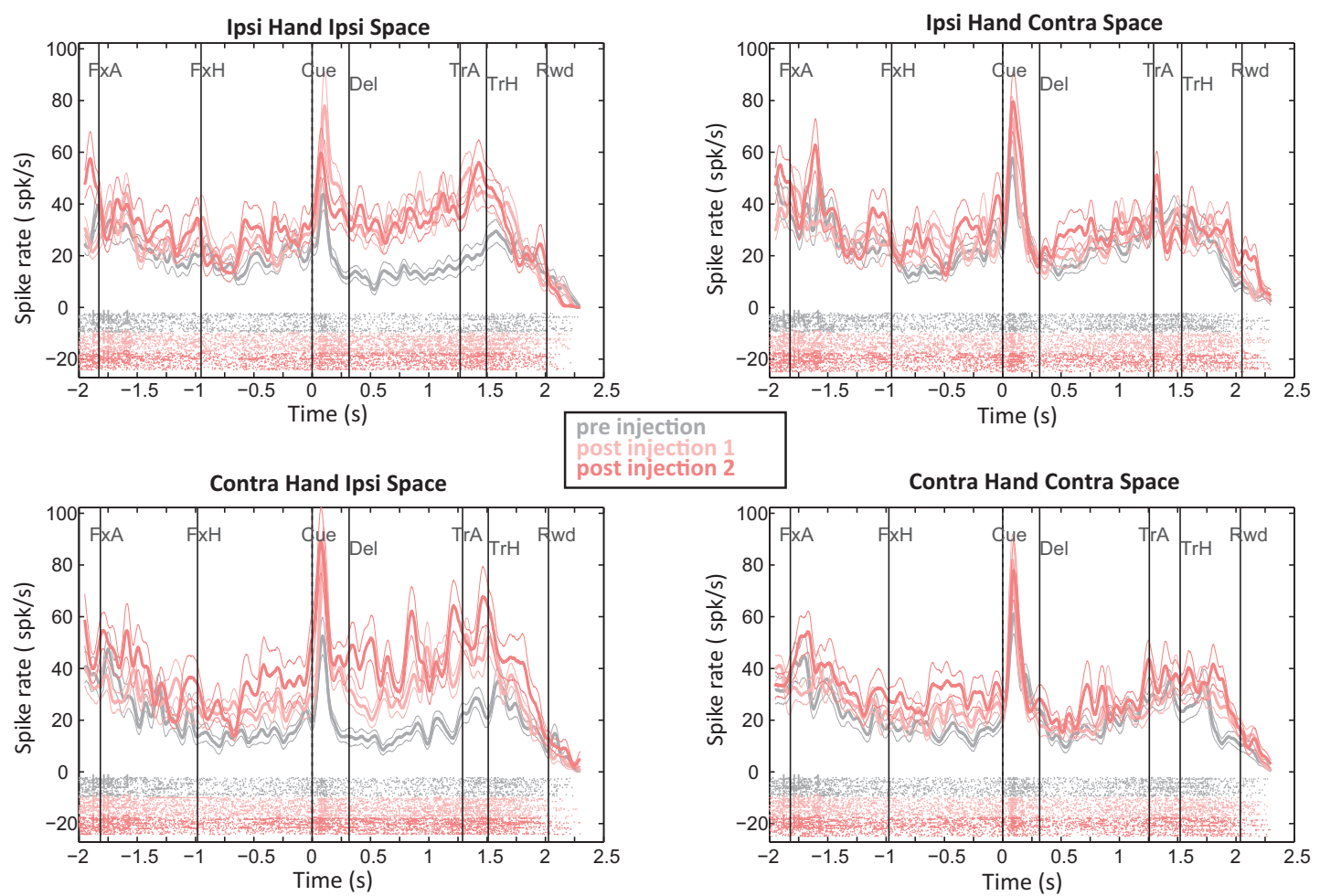

b) Unit “Lin_20171023_12" right LIP (Dissociated saccade task)
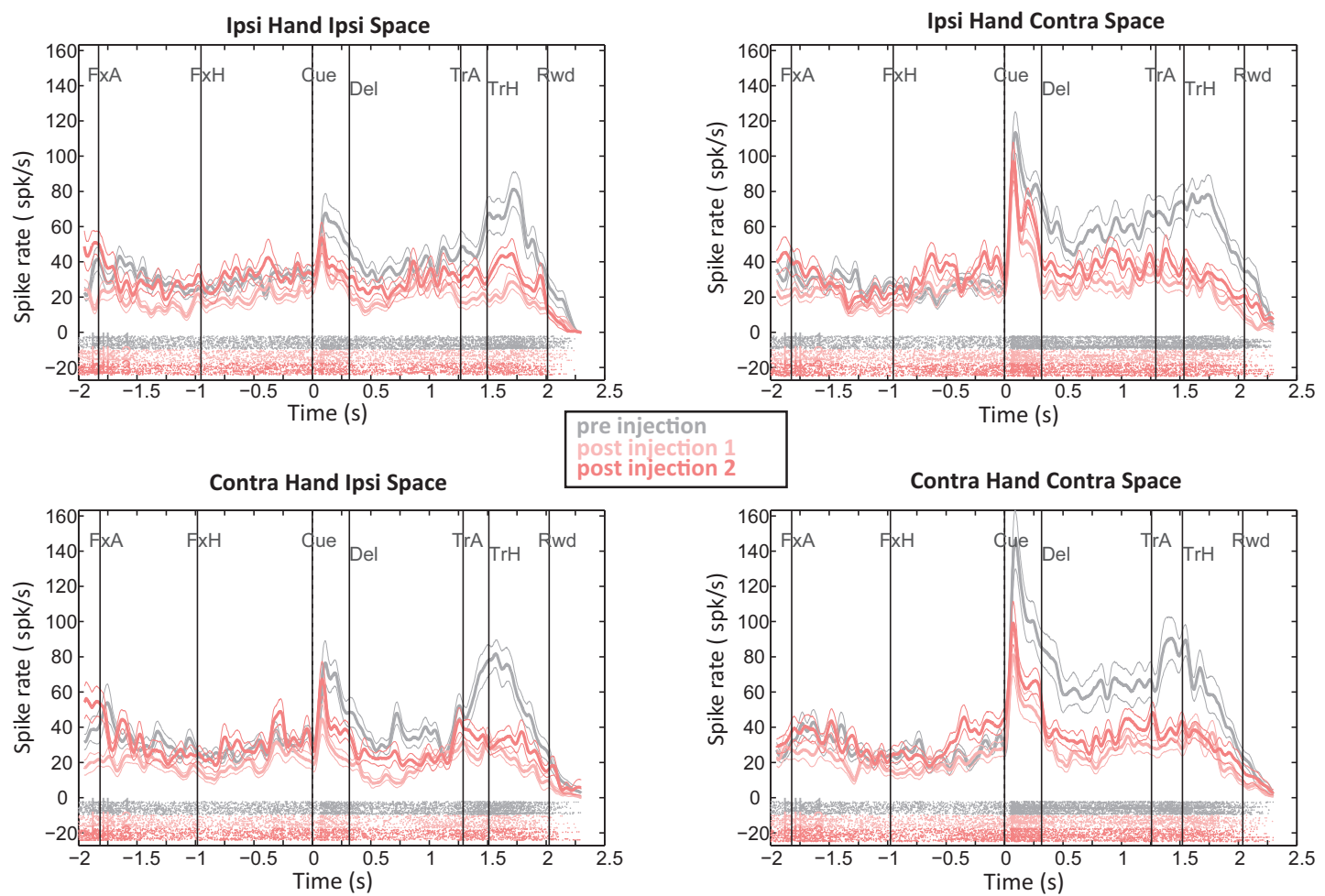

Fig. 3.24 Effect of pulvinar inactivation on the firing rate of two example units in right LIP (a and b). Each panel illustrates effect in one task condition. All PSTHs are aligned to the cue presentation (time zero). Each color represents averaged aligned PSTH across successful trials in one block (gray: pre injection block (control), light red: first block after inactivation, dark red: second block after inactivation). Ipsi and Contra are relative to the inactivated hemisphere (right). 


\section{Inactivation modulation index (IMI) for the units in right LIP ( $=27)$, dissociated saccade task}

a) Fixation hold period

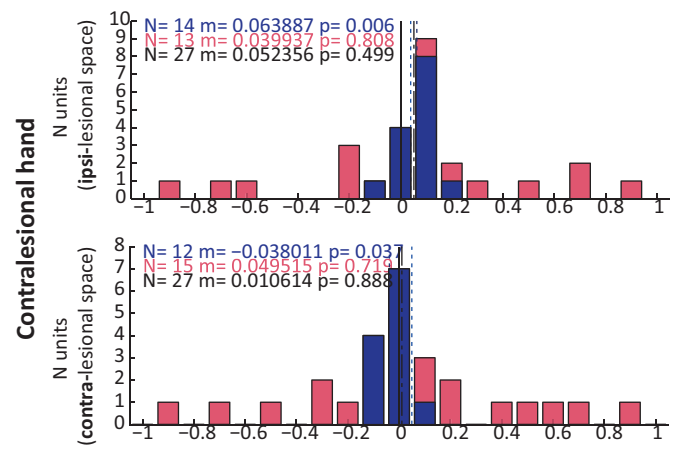

\section{b) Cue period}

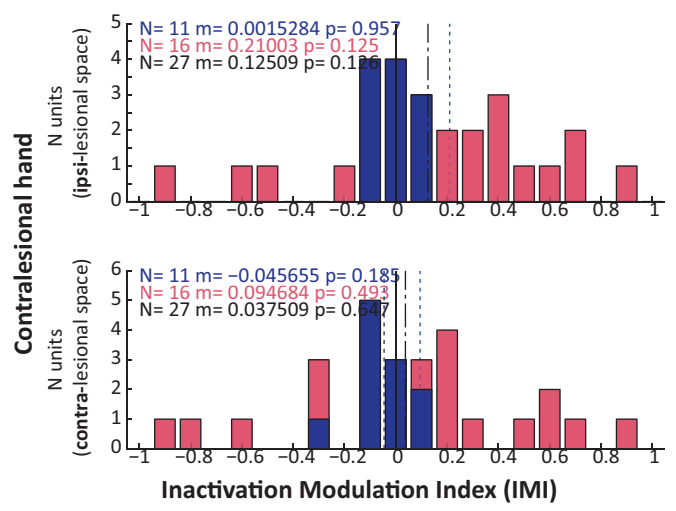

\section{c) pre-reach period}

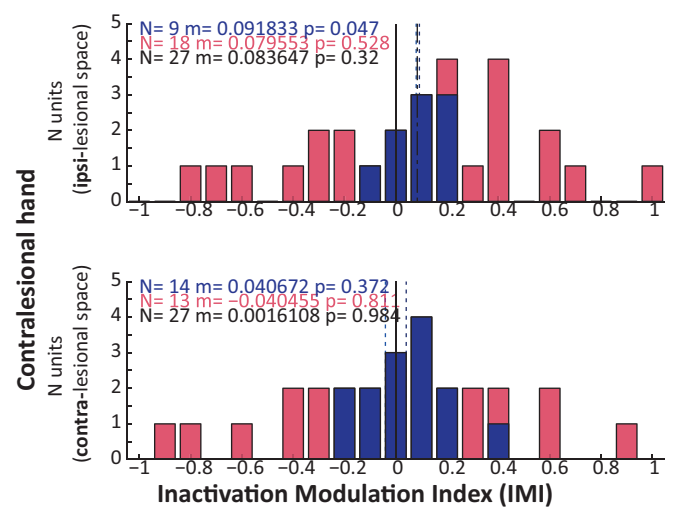

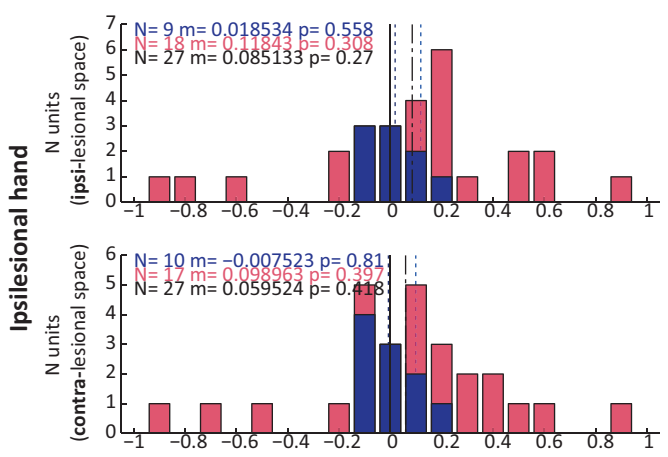

Inactivation Modulation Index (IMI)
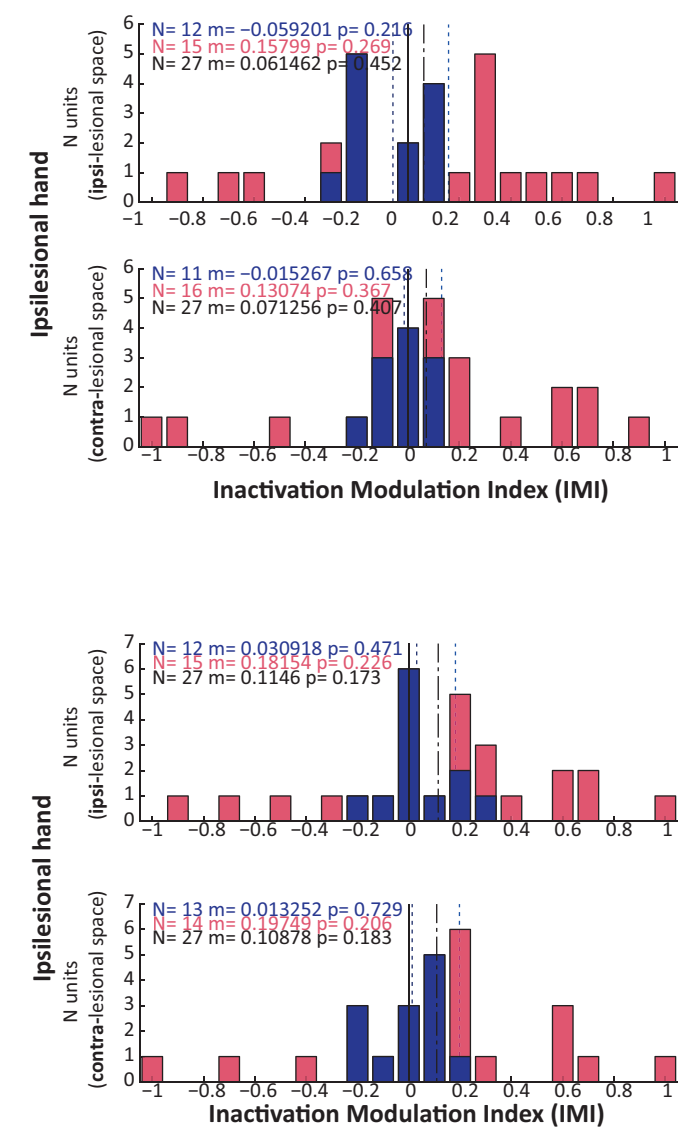

Fig. 3.25 Inactivation modulation index (IMI) for the population of units recorded from the LIP area in right (inactivated) hemisphere. IMI was calculated by subtracting average firing rate in the control trials from the inactivation trials and divided by the sum of two $(\mathrm{IMI}=(\mathrm{C}-\mathrm{I}) /(\mathrm{C}+\mathrm{I})$ ). IMI was computed for each task period (fixation hold $(300-0 \mathrm{~ms}$ before cue onset), cue (50-150 ms after cue onset), pre-saccade (300-10 ms before saccade onset) and each condition (ipsilesional hand, ipsilesional space, contralesional hand, contralesional space) separately. Unpaired ttest was applied between trials in the pre- versus post-injection to get the significant modulation. Dashed vertical lines illustrate means of IMI for the whole population, for the units with significant modulation (red) and for the units with not significant modulation (blue). Bold vertical line just indicate IMI=zero. Negative IMI values indicate decrease of firing rate and positive IMI values means increase of firing rate after inactivation. 
Inactivation modulation index (IMI) for the units in left LIP $(n=23)$, dissociated saccade task

\section{a) Fixation hold period}

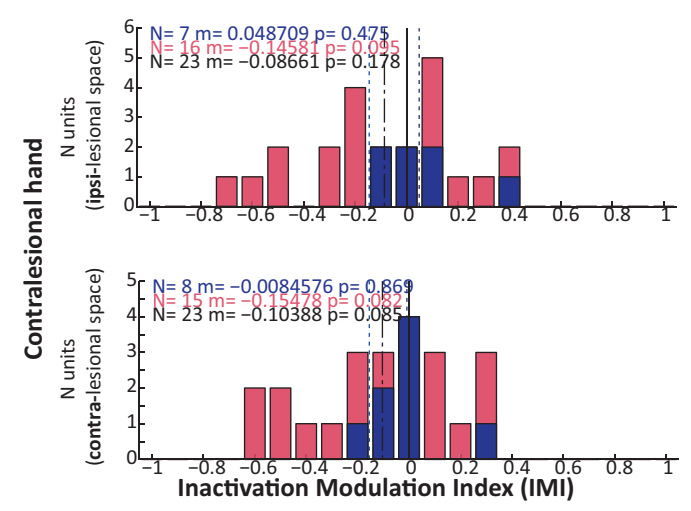

\section{b) Cue period}

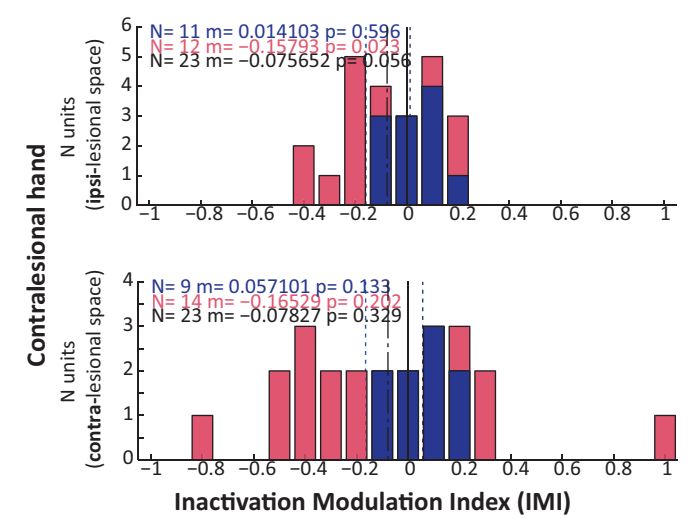

c) pre-reach period

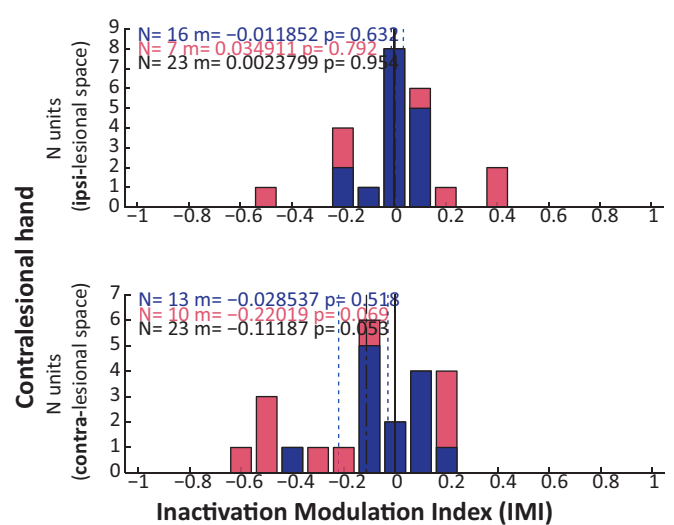

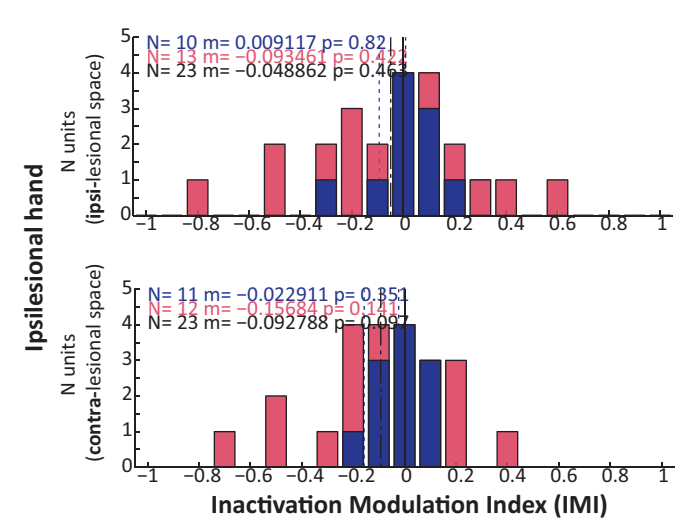
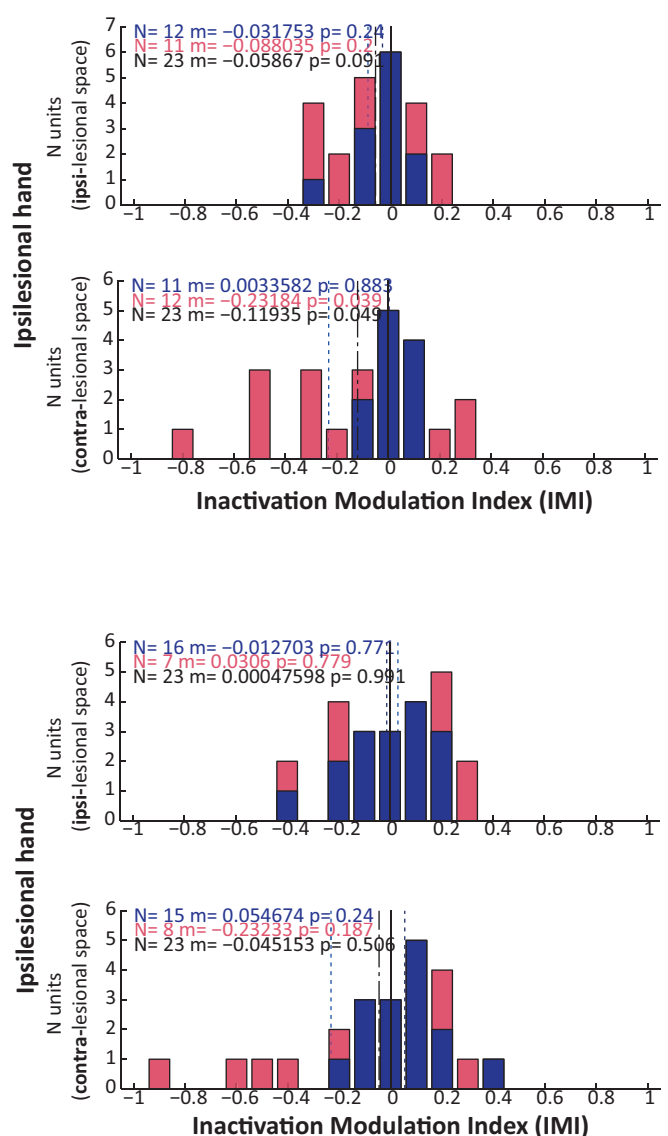

Fig. 3.26 Inactivation modulation index for the population of units recorded from the LIP area in left (intact) hemisphere. Specifications similar to Fig. 3.25. 


\subsubsection{Did pulvinar inactivation change LFP power in LIP similarly to MIP?}

Similar to the MIP recordings and pulvinar inactivation, here we asked whether pulvinar has a direct or indirect influence on the parietal LFP signal. If it would be the case then pulvinar inactivation causes significant changes in the LIP LFP measures such as power. We analyzed the data collected from four sessions. In total we recorded simultaneously 39 LFP sites (19 left hemisphere and 20 right hemisphere). In the following sections we will show first the power spectrum in the intact (pre-injection) trials averaged across sites and then we show how right pulvinar inactivation affects the power in different frequency bands and periods of the task.

\section{Power spectrum in the intact state}

Fig. 3.27 shows the averaged normalized time-frequency representations across instructed trials in the pre-injection block (intact state) across 20 LFP sites in the right (a) and 19 left hemisphere (b). In the right hemisphere, power in the alpha and lower beta increases during fixation hold period. This increase is weaker in the high beta band. Following cue presentation beta and gamma band power increases while alpha power decreases. Later in time during delay and go epoch beta shows a mild decrease in power but alpha power strongly increases. As the monkey performs the saccade gamma power increases and reversely power in the lower bands (theta, alpha, low beta) strongly decrease.

Except for significant ipsilesional hand-selectivity in the late delay period in the delta band, we do not see any other strong hand-selectivity (FDR corrected p-values: IHICSd 0.0137; CHICSd 0.0069; ICHdIS 0.0059; ICHdCS 0.0041). In contrast, this population of LFP sites in area LIP shows significant contralesional cue response in the high beta and gamma bands, stronger for the ipsilesional hand than contralesional hand. In addition, there is a significant ipsilesional space-selectivity in the delta and theta bands during the late delay and pre-movement periods for the ipsilesional hand and only during movement for the contralesional hand. 


\section{a) Average pre-injection z-scored spectrograms across sites (right hemi)}
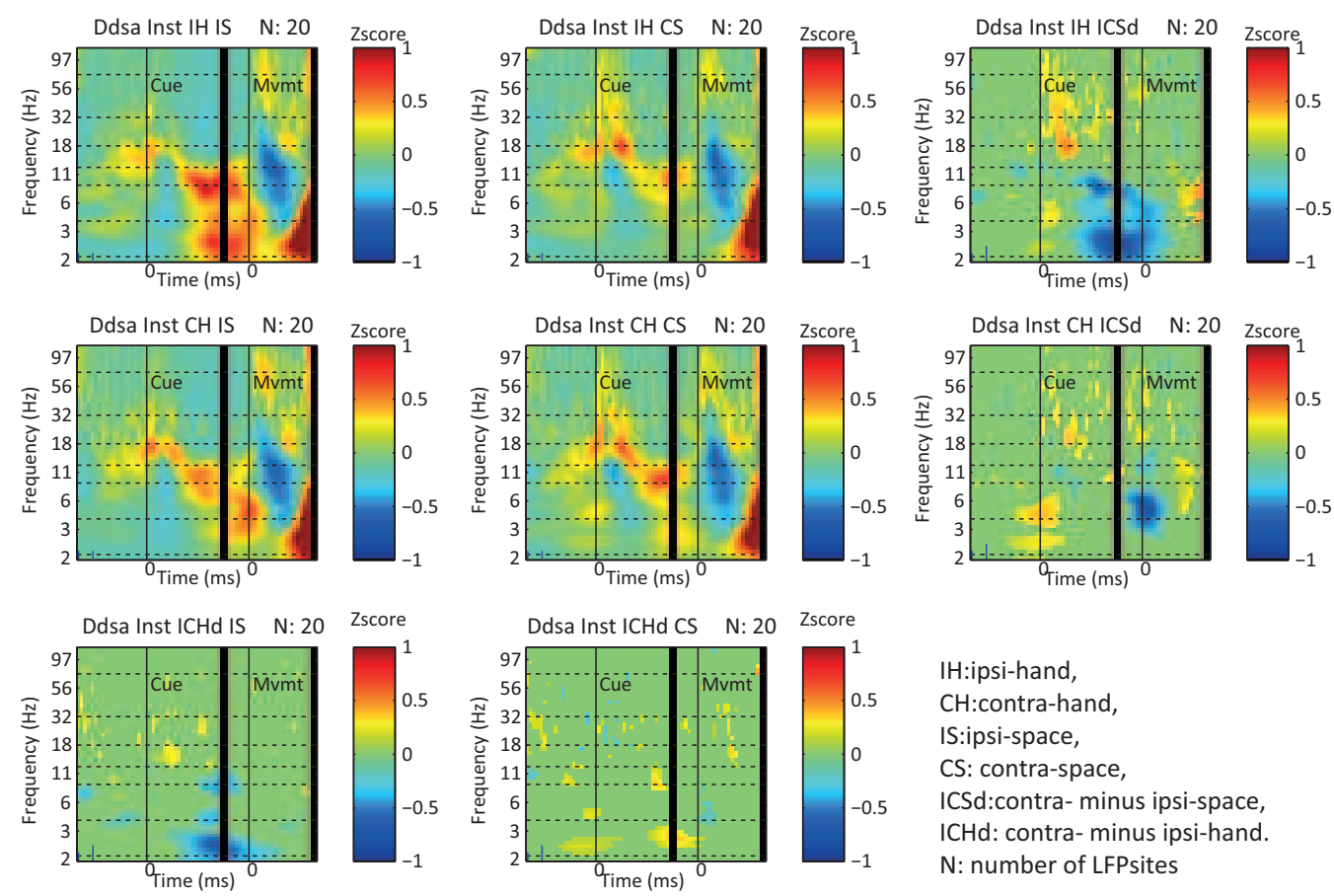

IH:ipsi-hand,

$\mathrm{CH}$ :contra-hand,

IS:ipsi-space,

CS: contra-space,

ICSd:contra- minus ipsi-space,

ICHd: contra- minus ipsi-hand.

$\mathrm{N}$ : number of LFPsites

\section{b) Average pre-injection z-scored spectrograms across sites (left hemi)}
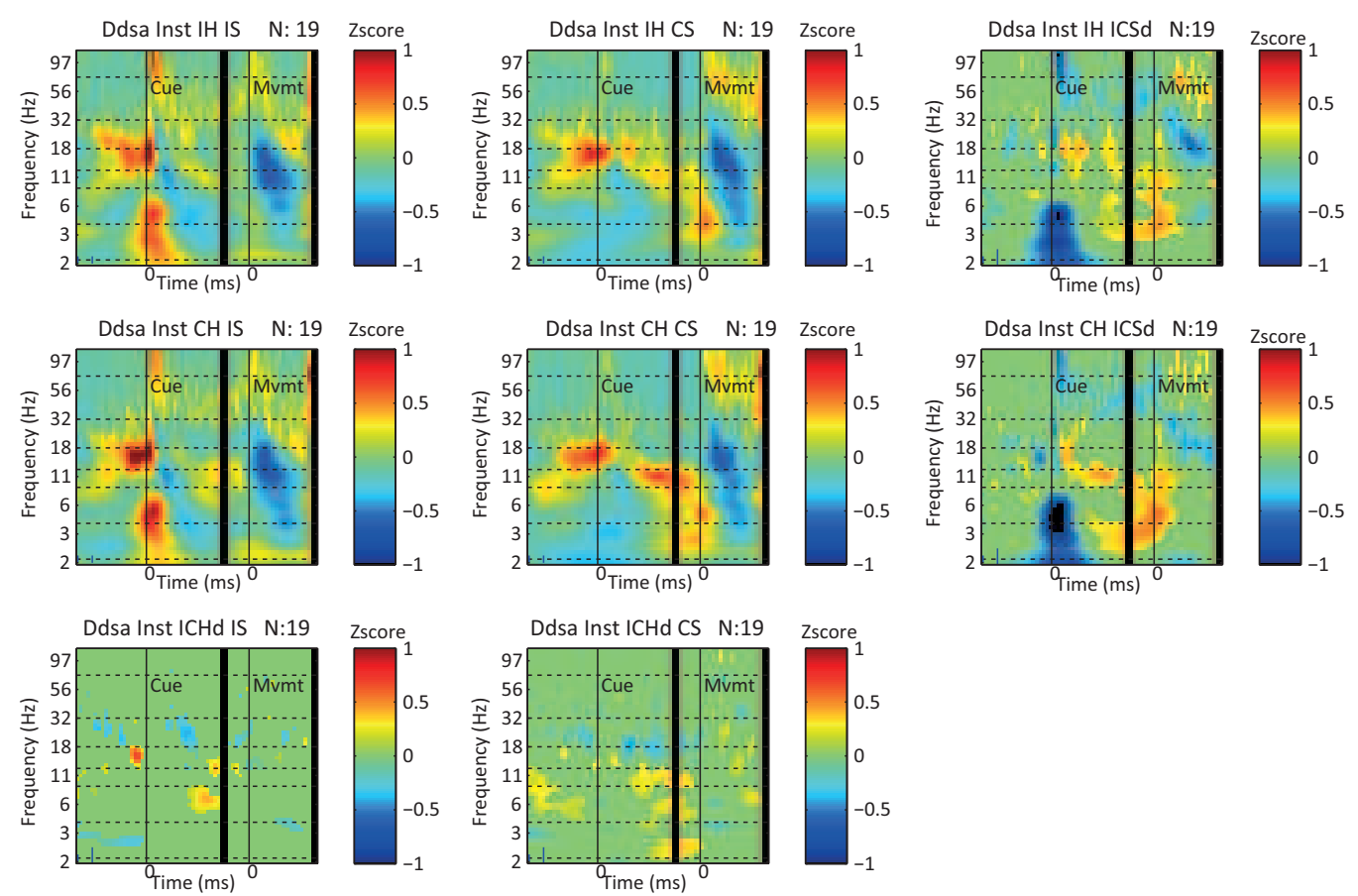

Fig. 3.27 a) Normalized (z-score) power spectrograms in the pre-injection block averaged across LFP sites in LIP in the right hemisphere(a) and left hemisphere (b). Monkey performed the instructed trials of dissociated delay saccade task. Format similar to Fig. 3.11. Ipsi and contra relative to the to-be-inactivated hemisphere 


\section{Averaged pre-injection z-scored spectrograms (choice trials)}

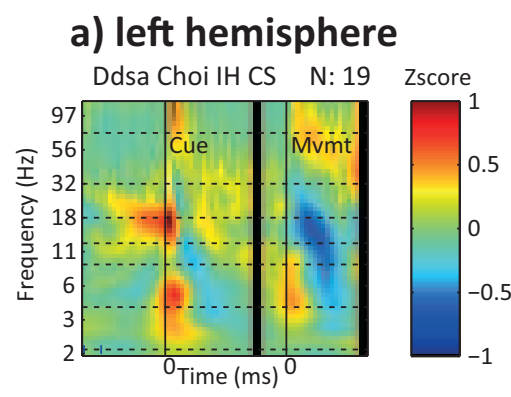

\section{b) right hemisphere}
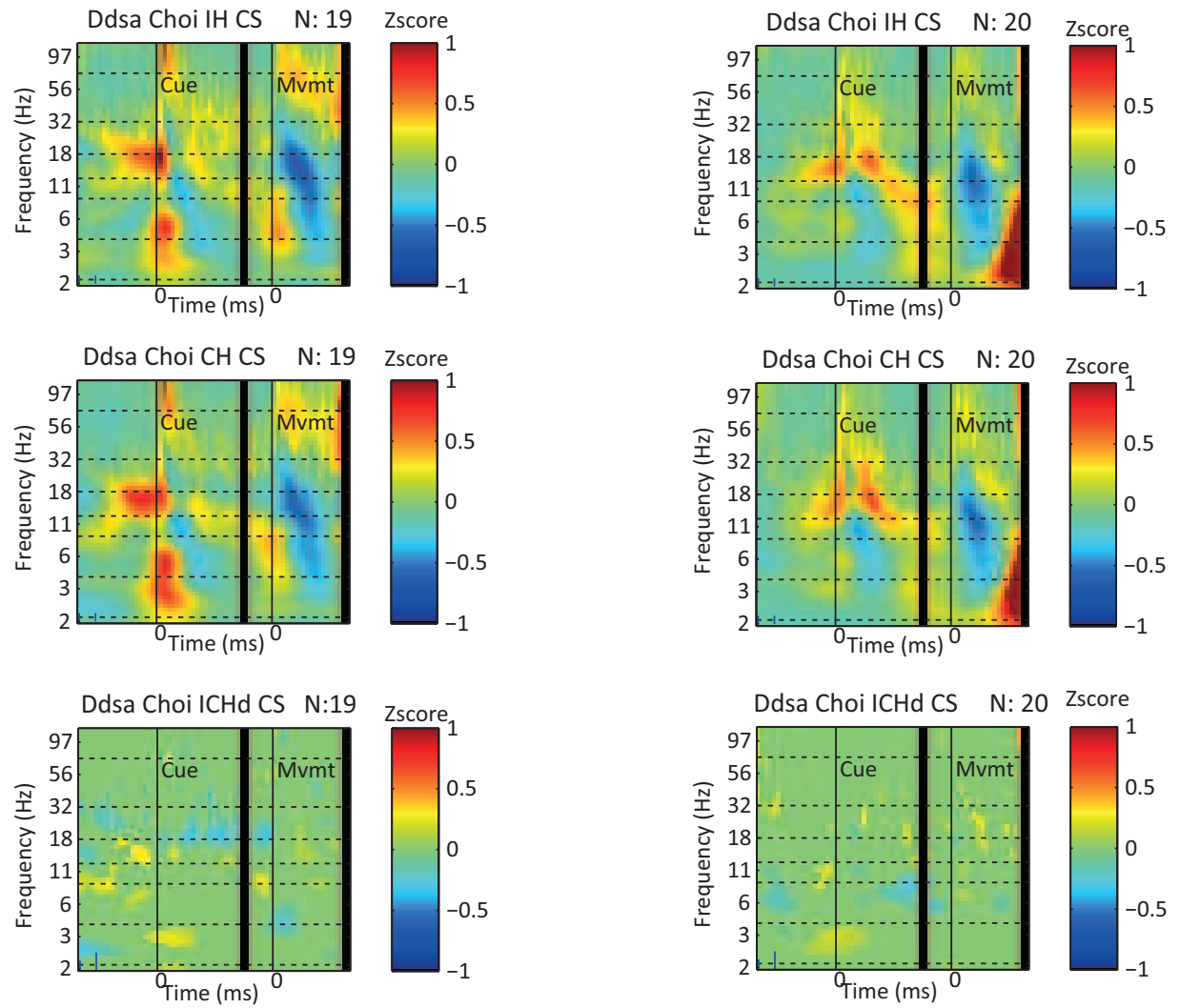

Fig. 3.28 Similar to Fig. 3.27 but for choice trials. The conditions for ipsilesional hand (IHIS, CHIS, IHICSd, CHICSd) are missing since the monkey didn't perform enough trials $(>10)$.

In the left hemisphere we see a general similar pattern. The most prominent frequencies are the beta band during fixation and delay and the gamma band during cue and movement. But there is slightly different pattern in selectivity maps compared to the right hemisphere (FDR corrected p-values: IHICSd 0.0180; CHICSd 0.0175; ICHdIS 0.0039; ICHdCS 0.0088). This population of sites shows significant ipsilesional cue response in the high gamma as well as delta and theta bands. In addition, these lower bands have a significant contralesional space-selectivity during delay, preand peri-movement for both hands, but stronger with the contralesional hand. Interesting is that in none of the two hemispheres we see any hand-selectivity in the beta range.

Figure. 3.28 illustrates the same analysis for the choice trials. Since monkey was selecting predominantly contralesional targets, there is not enough trials to show to representation for ipsilesional targets. As it is obvious from this figure the general pattern is the same as in instructed trials.

\section{Changes of LIP power spectrum within $\sim 2$-3hours after pulvinar inactivation}

We first examined the effects of the right pulvinar inactivation on the normalized power spectrum in the trials of the first block after injection. The effect size was computed on the same LFP sites as in the pre-injection block (intact state; left hemisphere 19 and right hemisphere 20 sites). We did here 
the same analysis as explained for Fig. 3.13. Since the observed effects was similar to the effects in the second block of trials (as it was in the MIP recordings), here we show only effects on the second block after injection.

In the inactivated right hemisphere (Fig. 3.29), power increased through the whole trial in the lower frequency bands (delta, theta and alpha) significantly (FDR corrected p-values: IHIS 0.0267; IHCS 0.0258; CHIS 0.0258; CHCS 0.0244; IHICSd 0.0021; CHICSd 0.0042; ICHdIS 0.0055; ICHdCS 0.0031) (similar to MIP recordings; see Fig. 3.14). This increase was highest in the late delay (del) and target acquisition (tgo) epochs in the delta and theta in which we observed the high power in the pre-injection block. In contrast, while alpha power did not change in any condition, beta power decreased significantly in all conditions (slightly higher for conditions in which ipsilesional hand was involved). This decrease was more prominent during the fixation hold period (especially for the ipsilesional hand). In addition, the power in gamma range did not change significantly in any epoch. We also computed the effect size in each epoch and frequency band (Fig. 3.29(b)) as we did explain previously. In this analysis we parse the whole spectrogram to a smaller matrix and apply a cruder statistic (ttest without FDR correction). This summarizes the effects in the most important epochs of the trial, which replicates nicely the effects in the spectrograms. In general, we see three main groups of frequency bands affected by the pulvinar inactivation in the same hemisphere. Lower bands get increased while beta decreases and gamma does not change much. These seem to be not so much epoch dependent.

But in the opposite hemisphere (intact), we observed a different effect (Fig. 3.30). The lower frequency bands decreased significantly only for the contralesional hand and mostly during delay and pre-saccadic periods (FDR corrected p-values: IHIS 0.0146; IHCS 0.0113; CHIS 0.0170; CHCS 0.0183; IHICSd 0.0022; CHICSd 0.0043; ICHdIS 0.0030; ICHdCS 0.0053). In this hemisphere, alpha and lower beta band power decreased in all conditions. This effect deviates from the inactivated hemisphere because there we had the decrease also in high beta but no changes in alpha range. Interestingly while gamma in right hemisphere did not change at all, in the intact hemisphere, it increased during delay and pre-saccadic time only for the ipsilesional targets. This effect is somehow similar to what we saw in MIP sites (see Fig. 3.15). One important point is that in neither right nor left hemisphere we observed any profound changes in the selectivity maps. Last but not least, Figure 3.31 shows the effects during the choice trials. Generally, we saw absolutely same results in choice as in instructed trials with a similar strength. One explanation for that is that for this monkey choice trials can be treated as instructed since he had strong bias to select targets congruent to the acting hand-space. 
a) Changes in spectrograms: post $_{2}$-preinjection (inactivated right hemisphere)
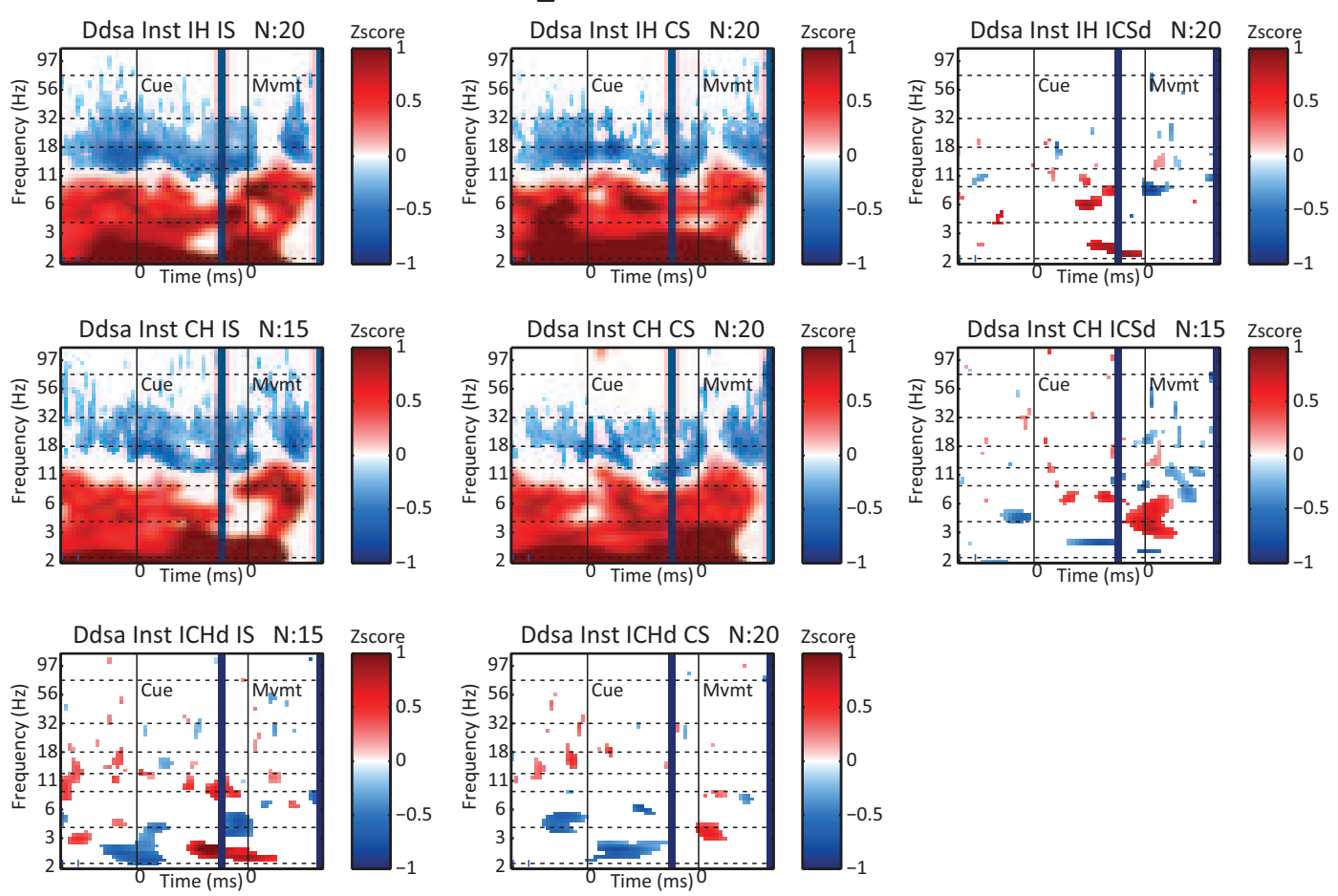

\section{b) Effect-size : post $_{2}$-preinjection (inactivated right hemisphere)}
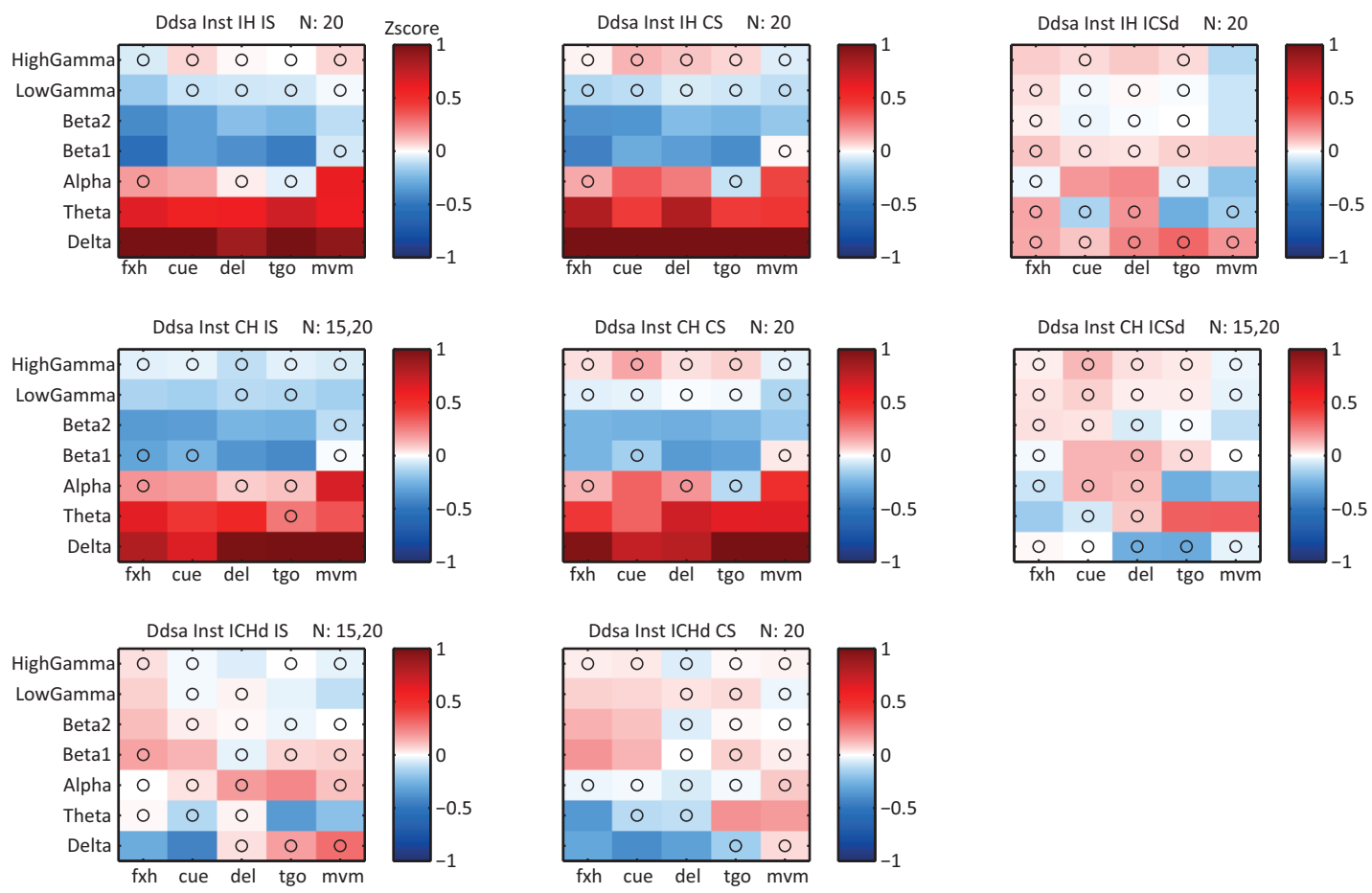

Fig. 3.29 (a) Significant changes in the averaged normalized power (spectrograms) after inactivation in the second block of instructed trials ( 2-3 hour after injection) across sites in the LIP right hemisphere. Similar to Fig. 3.14(a). Part (b) shows the effect size of inactivation in each frequency band and epoch separately. Similar to figure 3.16. Empty circles show non-significant bins. 
a) Changes in spectrograms: post $_{2}$-preinjection (intact left hemisphere)
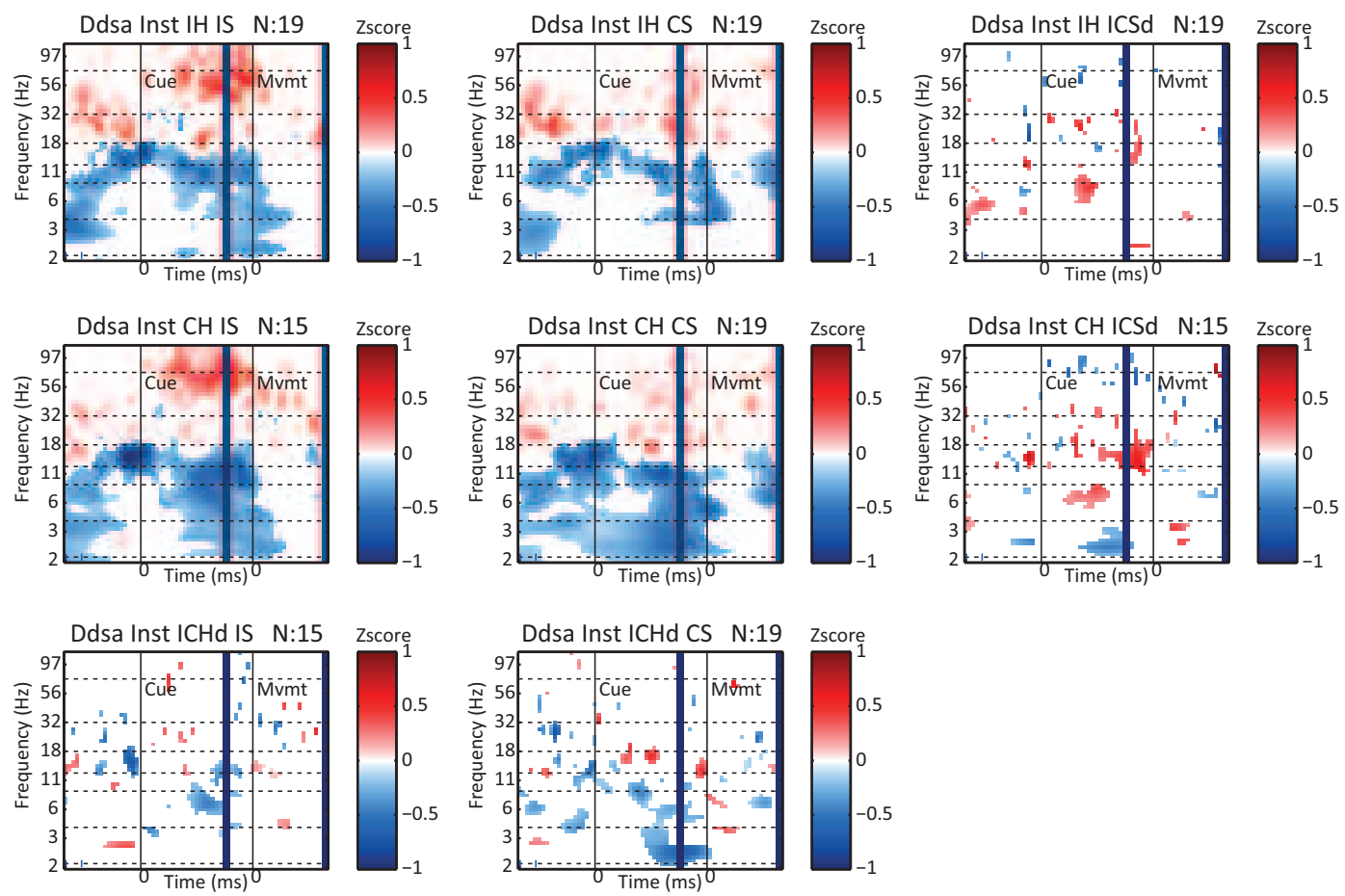

\section{b) Effect-size : post $_{2}$-preinjection (Inst trials, sites in intact left hemisphere)}
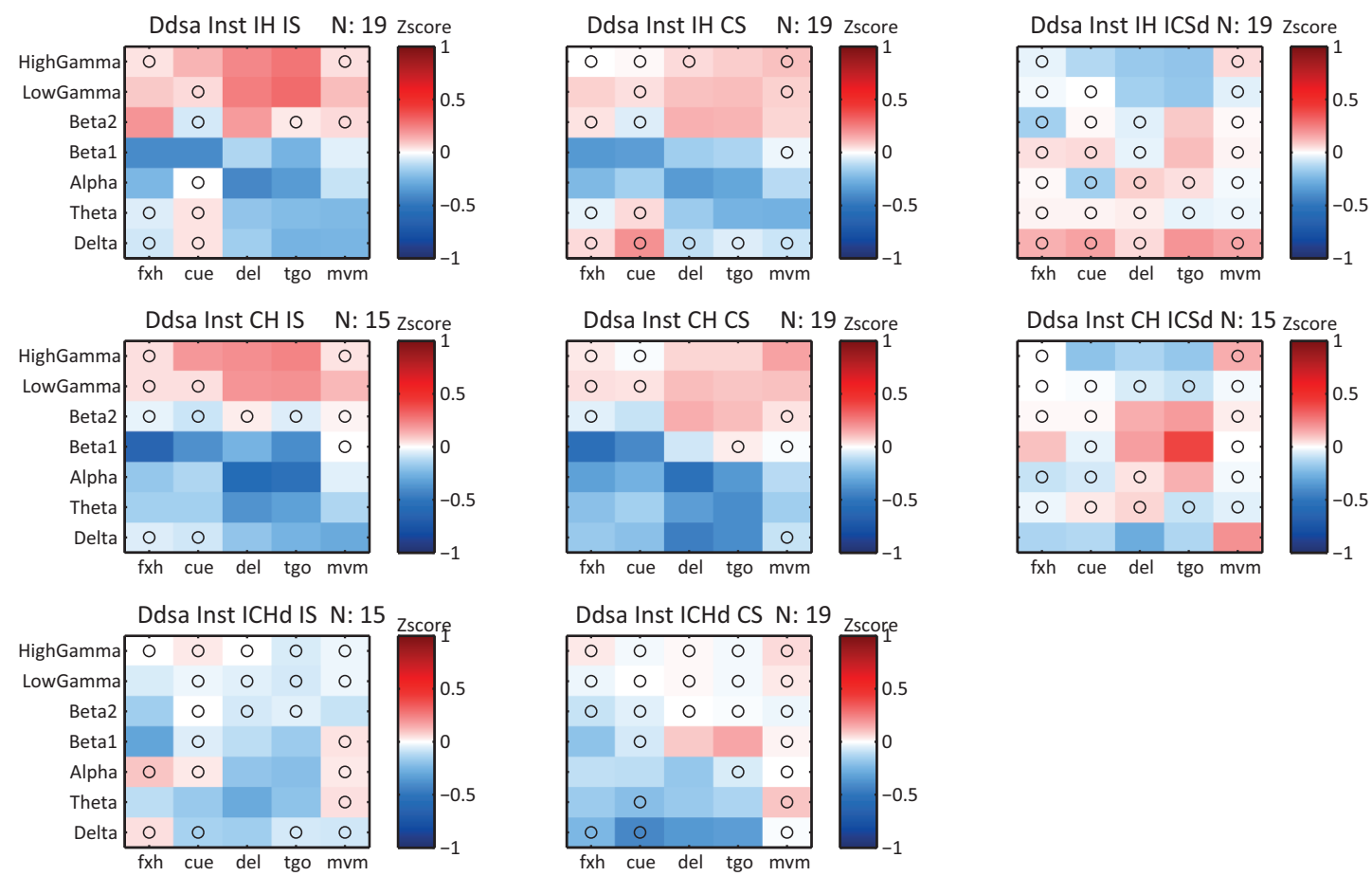

Fig. 3.30 (a) Significant changes in the averaged normalized power (spectrograms) after inactivation in the second block of instructed trials ( 2-3 hour after injection) across sites in the LIP left hemisphere. Similar to Fig. 3.14(a). Part (b) shows the effect size of inactivation in each frequency band and epoch separately. Similar to figure 3.16. Empty circles show non-significant bins. 


\section{Effect-size: post ${ }_{2}$-preinjection (Choice trials)}

a) across sites in left hemi (intact)
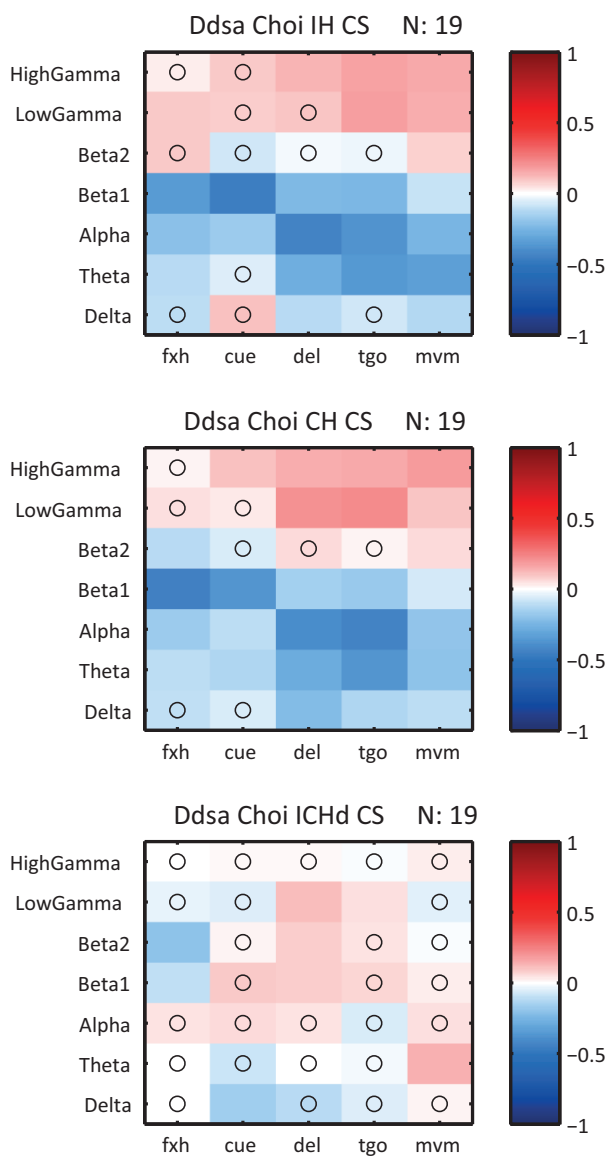

b) across sites in right hemi (inactivated)
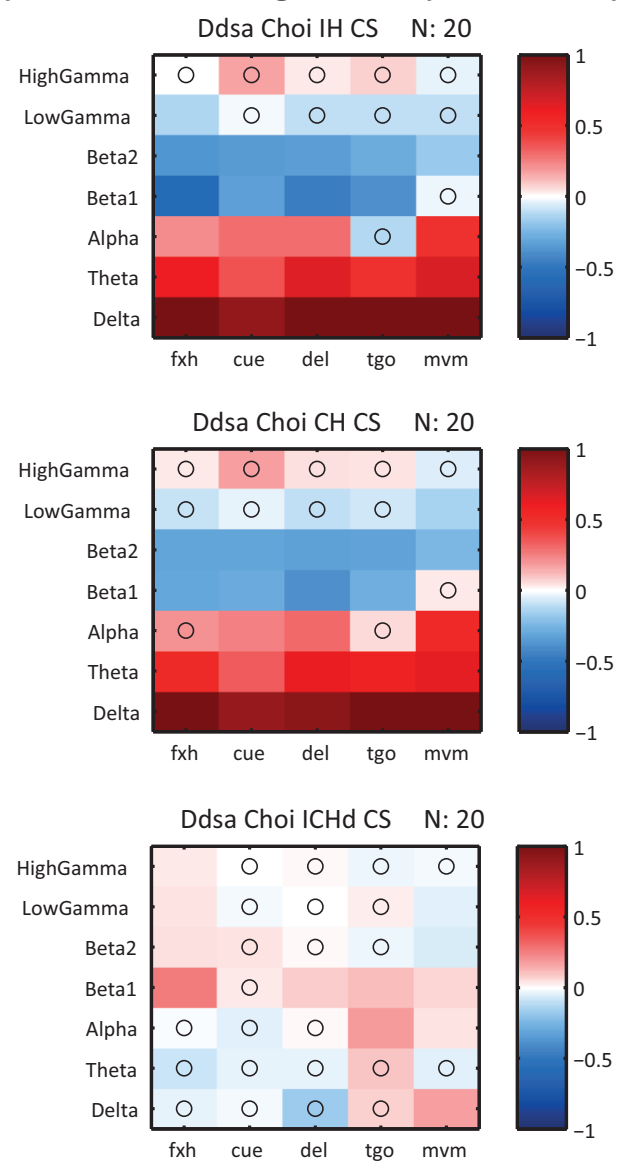

Fig. 3.31 Effect size of inactivation on the second block of choice trials, similar to Fig. 3.17. a) averaged across sites in the left hemisphere and (b) right hemisphere. 


\subsubsection{Behavioral effects of unilateral pulvinar inactivation in the delay saccade task}

As previously explained, in this experiment monkey performed dissociated delayed saccade (Dds) task. The beginning of each trial until cue presentation was the same as the delayed reach (Ddr) task but since we separated tasks by blocks we assume that monkey knew from the first few trials that he will perform that task in all trials. Basically this assumption means that also in the fixation acquisition and fixation hold period he is planning to eventually do the saccade but not reach toward spatial target.

Fig. 3.32 shows the behavioral effects after inactivation. Panel (a) shows that overall performance dropped significantly after pulvinar inactivation for both instructed (pre-injection: $M=89.8 \mathrm{~ms}$, SEM=1.65ms; post-injection: $M=50.7 \mathrm{~ms}, S E M=3.6 \mathrm{~ms}$; paired ttest $\mathrm{df}=3 \mathrm{p}=0.0001$ ) and choice trials (pre-injection: $\mathrm{M}=93.28 \mathrm{~ms}$, SEM=1.4ms; post-injection: $\mathrm{M}=37.9 \mathrm{~ms}$, SEM2.5ms; paired ttest $\mathrm{df}=3$, $\mathrm{p}=0.001$ ). Similar to the effects in the delayed reach task, we observed significant increase in monkey's initial reaction time to release hand from sensor after inactivation Fig. 3.32(c) (contralesional hand: $M=94.7 \mathrm{~ms}, S E M=24.7 \mathrm{~ms}$; paired ttest $\mathrm{df}=3 \mathrm{p}=0.04$ versus ipsilesional hand: $M=148.3 \mathrm{~ms}$, SEM23.7ms; paired ttest $\mathrm{df}=3, \mathrm{p}=0.01$ ).

Similarly, pulvinar inactivation increased the hand movement toward fixation spot Fig. 3.32(d) (with contralesional hand: $\mathrm{M}=71.7 \mathrm{~ms}, \mathrm{SEM}=10.9 \mathrm{~ms}$; paired ttest $\mathrm{df}=3, \mathrm{p}=0.01$ and with ipsilesional hand: $M=53.2 \mathrm{~ms}, \mathrm{SEM}=4.6 \mathrm{~m}$; paired ttest $\mathrm{df}=3, \mathrm{p}=0.002$ ). This means that the monkey was slower to acquire the fixation with the hand.

In addition, interestingly the saccade RT toward contralesional spatial target increased after pulvinar inactivation, in both choice trials (Fig. 3.32(d); CHCS: M=100.9ms, SEM=17.2ms, p=0.01; IHCS: $\mathrm{M}=88.9 \mathrm{~ms}, \mathrm{SEM}=26.2 \mathrm{~ms}, \mathrm{p}>0.05$; $\mathrm{p}$-values calculated by paired ttest, $\mathrm{df}=3$ ) and in instructed trials (Fig. 3.32(e); CHCS: $\mathrm{M}=83.3 \mathrm{~ms}, \mathrm{SEM}=12.2 \mathrm{~ms}, \mathrm{p}=0.01$; $\mathrm{CHIS}: \mathrm{M}=52.2 \mathrm{~ms}$ SEM=4.5ms $\mathrm{p}=0.002$; IHCS: $\mathrm{M}=65.7 \mathrm{~ms}$ SEM=9.1ms $\mathrm{p}=0.008$; IHIS: $\mathrm{M}=18.3 \mathrm{~ms}$ SEM=18.7ms $\mathrm{p}>0.05$; $\mathrm{p}$-values calculated by paired ttest, $\mathrm{df}=3$ ).

We also addressed whether monkey's spatial choice preference changed after pulvinar inactivation. In the preinjection block, the monkey made saccades almost always toward contralesional space when there was a free-choice decision (Fig. 3.32(f); M=98.1\%, SEM=0.9). Interestingly, after pulvinar inactivation, the monkey's choice bias toward contralesional space decreased (Fig. 3.32(h); fixation with contralesional hand: $\mathrm{M}=8.0 \%, \mathrm{SEM}=4.0 \%, \mathrm{p}=0.18>0.05$; fixation with ipsilesional hand: $\mathrm{M}=6.8 \%, \mathrm{SEM}=3.3 \%, \mathrm{p}=0.17>0.05)$. In addition, his performance slightly dropped only for target in contralesional space (Fig 3.32(i); contralesional space: $\mathrm{M}=10.5 \%, \mathrm{SEM}=4.3 \%, \mathrm{p}=0.12>0.05$; ipsilesional space: $\mathrm{M}=2.1 \%, \mathrm{SEM}=1.8 \%, \mathrm{p}=0.39>0.5)$. 
a)

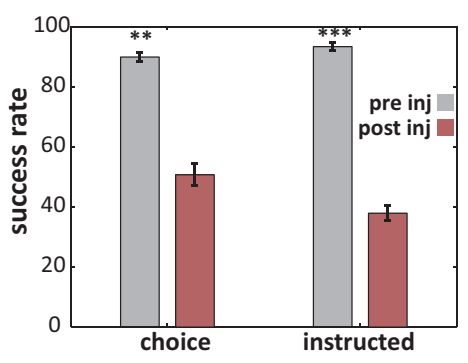

b)

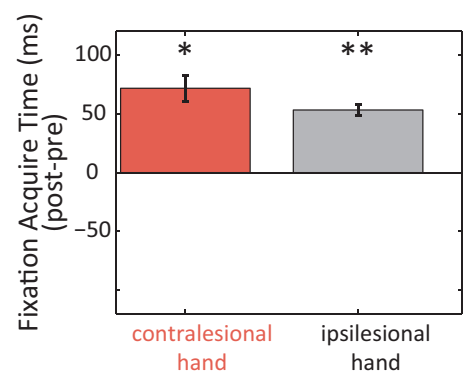

c)

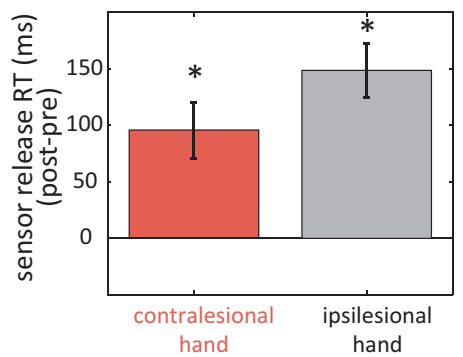

d)

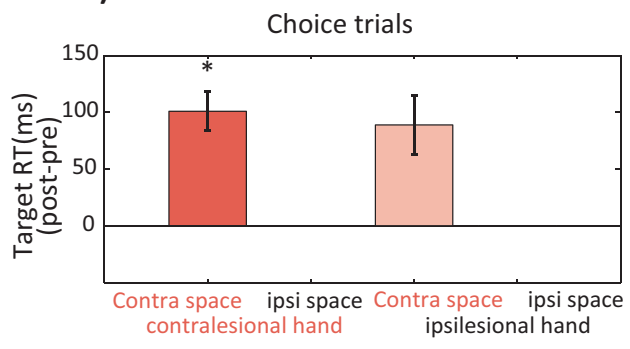

f)

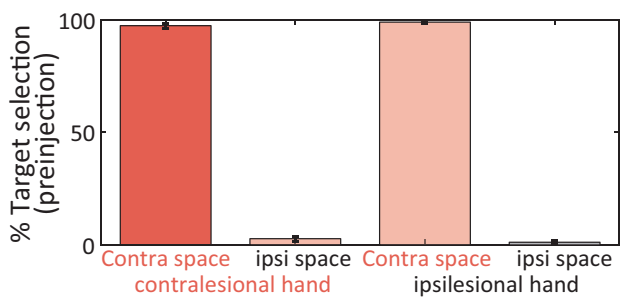

h)

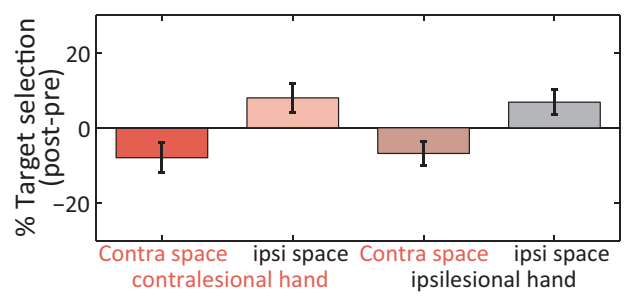

e)

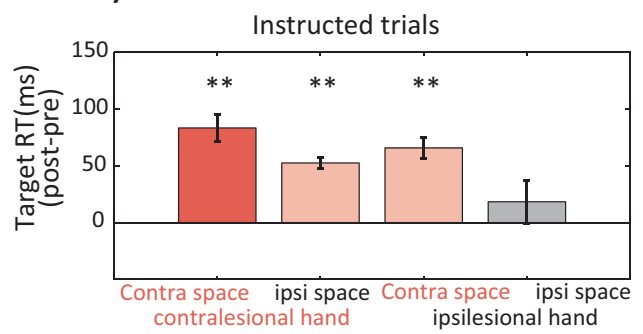

g)

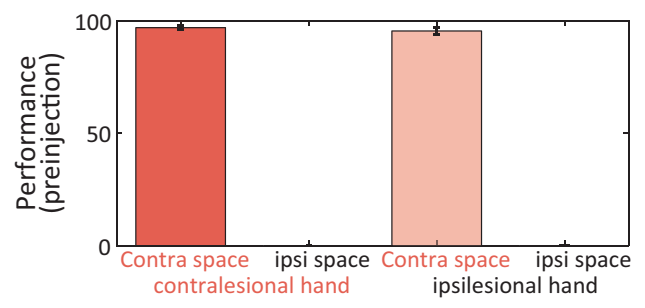

i)

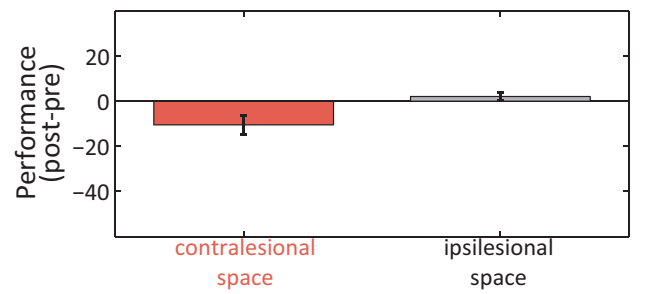

Fig. 3.32 Behavioral effects of right pulvinar inactivation while monkey performed dissociated delayed saccade task. a) Overall performance in instructed and choice trials. b) and c) Changes of movement time from sensor to fixation and also sensor release time after inactivation, respectively. (d) Target reaction time in choice trials. (e) Target reaction time in instructed trials (f) Target selection in the pre-injection (intact state) in choice trials (g) Performance of target selection in the pre-injection choice trials (h) Changes in choice target selection after inactivation (i) Changes of performance in choice target selection after inactivation. Paired ttest applied to obtain significance between pre and post injection. $* \mathrm{p}<0.05 * *$ $\mathrm{p}<0.01 * * * \mathrm{p}<0.001$. Errorbars are the standard error of mean across four sessions (dof $=3$ ). 


\subsection{Discussion}

The functional role of dorsal pulvinar in visually-guided reach and saccade movement goals is still largely unknown and the available knowledge is mostly rely on lesion and inactivation studies (Wilke et al., 2018, 2010) as well as a few neurophysiological recordings (Acuña et al., 1986). In addition, it is unknown whether its potential functional role is through mediating the neuronal activity in the frontoparietal network for goal-directed behavior (Gail et al., 2009; Klaes et al., 2011; Pesaran et al., 2008; Wong et al., 2016).

LIP and MIP are two nodes within PPC separated by IPS, which have different anatomical connections to sensory and frontal areas and are associated with goal-directed behavior in an effectorspecific manner. LIP is more responsive to planning for a visually-guided saccade target but MIP for visually-guided reaches than saccades (Andersen \& Cui, 2009; Caminiti et al., 2015; Kubanek et al., 2013; Snyder et al., 1997). Although several studies have shown this effector-specificity in different parietal parcels but the exact functional role of them is still not fully understood. For example it has been shown that mixture of reach and saccade goal encoding in the inferior parietal areas (including LIP) is much more stronger than in the superior parietal areas (such as MIP and PRR) (Battaglia-Mayer et al., 2016) and also it has been reported that spike-field coherent activity in LIP predicted saccade metrics only when it was accompanied with a reach but not alone (Dean et al., 2012). These imply that the level of effector-specificity in the parietal cortex is different in different locations. Apart from that we know also both LIP and MIP are reciprocally connected with dorsal pulvinar Cappe et al. (2007); Hardy \& Lynch (1992). Given the current knowledge we expect that pulvinar mediate neuronal responses in LIP and MIP for goal directed effector-specific behavior differently.

We pharmacologically inactivated dorsal pulvinar and simultaneously recorded from MIP (or LIP) in both hemispheres, while a monkey performed dissociated delay reach (or saccade) task. We asked whether unilateral inactivation of dorsal pulvinar directly changes the hand- and space representations of neuronal activity in parietal areas and whether these potential changes vary across hemispheres. To best of our knowledge, this is the first study to understand the causal role of thalamic dorsal pulvinar on the reach- and saccade-related neuronal responses in cortical parietal areas by combining two experimental methods.

\subsubsection{Unilateral pulvinar inactivation caused mostly contralesional reach and sac- cade deficits}

In both tasks, after inactivation performance significantly dropped by about $30 \%$. Error rates were higher for contralateral than ipsilateral hand (not significant). In dissociated delay reach (Ddr) task, monkey did not show a general change in spatial preference after pulvinar inactivation, but when we separated the trials by hand, we found that he had a significant ipsilesional space preference only with the contralesional hand (hand-specific spatial preference). Consistently the main effect was the slowness in the movement timings with both hands but stronger with contralesional hand. At the beginning of the trial, the monkey was not only significantly slower to release his contralesional hand from proximity sensor, but also slower to move that hand toward the fixation spot. It is worth mentioning that he was also slow with ipsilesional hand but much less than contralesional 
hand. Moreover, from the fixation spot toward the spatial target, his movement time (MT) became significantly slower only with the contralesional hand to both side of space.

After each experiment ( 3-4 hours after drug injection) we tested his behavior in a controlled (out of experimental setup) condition but head-fixated in the monkey-chair. He was unwilling to use his contralesional hand to grab a piece of fruit when he had a choice to grab it with either arm although this was not observed after the experiment. Interestingly when the ipsilesional arm was constrained, he was able to grab the fruit with contralesional arm but not when both arms were free. Another effect that was also reported before (Wilke et al., 2018), was that monkey showed unusual and weird hand-shape after inactivation only in the contralesional hand. In addition, we often observed "self-grasping" (Wilke et al., 2010), i.e. ipsilesional hand grasped the contralesional hand. These might be due to the proprioceptive impairments which caused by the disruptions in sensory-motor integration through thalamocortical networks. In addition, while he showed rigidity and slowness when he had choice to move the arm and grasp a fruit but inside the cage he showed completely normal moving behavior. He could walk, run and jump normally. This could imply that the monkey was not impaired for the primary motor and visual processing.

In saccade task, monkey's performance after unilateral pulvinar inactivation dropped more for the targets in the contralesional space. In addition, he showed a slight increase in ipsilesional choice preference (no matter with which hand he fixated). And interestingly, his saccadic MT did not change after inactivation but his RT toward contralesional targets increased more than ipsilesional side. Our data overlaps with the previous studies with pulvinar inactivation or lesions (Wilke et al., 2018, 2010). Unilateral pulvinar inactivations causes ipsilesional saccadic and reach choice bias which could be balanced to normal after associate higher reward to contralesional targets (Wilke et al., 2013). Lesion or inactivation of superior parietal areas such as PRR and MIP affects severely the arm movement and grasping deficits. For example ipsilesional space or hand preference (Christopoulos et al., 2015b; Kubanek \& Snyder, 2015b; Padberg et al., 2010), increased target RT only with contralesional hand (Yttri et al., 2014), no effect on MT (Christopoulos et al., 2015b; Hwang et al., 2012; Kubanek \& Snyder, 2015b) but hypo- or hyper-metric reaches toward contralesional targets (Hwang et al., 2012; Kubanek \& Snyder, 2015b) or any target (Hwang et al., 2014). But on the other hand it causes effects only on saccadic parameters (e.g. RT) only when the lesion is bilateral (Battaglia-Mayer et al., 2013; Gaveau et al., 2008) not unilateral.

Lesions to parietal has been shown to cause symptoms called "optic ataxia" (OA) in which the main feature is the impairment in integration of directional visual information with arm movement signals toward a spatial target. Optic ataxia causes misreaches mostly with contralesional arm but when OA patients were allowed to look and reach (foveal reach) the deficit was reduced or alleviated. Our results show similarities with OA disorder which are reach related deficits but since monkey only performed extra-foveal reach and no foveal reach we could not properly test the similarities of our effects with OA (although according to other data from our lab, pulvinar inactivation causes increase in reach endpoint inaccuracy more with contralesional than ipsilesional hand).

On the other hand, our data with dissociated saccade task (only 4 sessions) resembles partially LIP inactivations (Kubanek \& Snyder, 2015b; Wardak et al., 2002; Wilke et al., 2012) with no hand related deficits but ipsilesional choice preference and increased target RT toward contralesional side. However, in our task (dissociated delay saccade) monkey had to perform a central reach (from the 
resting sensors toward the fixation spot) and keep the hand fixated until the end of the trial. In this task monkey had slower initial reaches after inactivation with both arms (data only from 4 sessions) which is not consistent with the effect with dissociated reach task. One explanation would be that monkey already knew he had to perform saccade toward spatial target and he had to just keep the hand fixated. Therefore, in this task the arm movement planning had different application (task-context) and consequently different neuronal functionality. It is worth mentioning that from data presented in previous chapter we showed that the beta band power during the fixation hold is different between dissociated reach and saccade tasks. In that respect it is expected to see no limb-specific effect.

\subsubsection{Unilateral pulvinar inactivation alters MIP spiking activity differently in oppo- site hemispheres}

We recorded MIP spiking activity simultaneously from both hemispheres. We asked whether unilateral pulvinar inactivation affected firing rates (FR) in opposite hemispheres differently. Therefore, we calculated how much the firing rate change after inactivation in each epoch and condition separately.

We observed consistent effects across different task epochs (with minor differences) in either hemisphere. At the population level, MIP units in the inactivated hemisphere were modulated in both directions. That means, while many units were up-modulated (increased their FR), many were also down-modulated (decreased in FR), so that as a population there was no significant change in FR. In spite of this, values of the modulation indices showed that there is a tendency toward decrease of FR, which means there were more down-modulated units than up-modulated. However, on the other side (intact left hemisphere), most of the units were significantly up-modulated (some unit with two or three time increased in FR), which leaded to a highly significant up-modulated population. This effect was consistent across all epochs and task conditions.

This unbalanced effect across hemispheres implies as if the intact hemisphere is released from a broad inhibition and that increased the firing rates generally. However, more investigation needs to be done to qualify the epoch-specificity of changes in firing rates. This fits to the model proposed to explain neglect syndrome (Kinsbourne, 1977) in which a dynamic competition between hemispheres through inhibitory interactions was supposed. In spite of this, the fact that callosotomy was used to reduce or diminish epileptic seizures, supports the excitatory role of interhemispheric connections through corpus callosum (Bloom \& Hynd, 2005). From the anatomical point of view, almost all callosal fibers are excitatory (Bloom \& Hynd, 2005; Palmer et al., 2012) but depending on the factors such as neurotransmitters and receptors it may function inhibitory or excitatory. In addition, it has been shown that local interneurons in cortex might be a key function of interhemispheric inhibition (Palmer et al., 2012).

The main inhibitory input to thalamic cells is from thalamic reticular nucleus (TRN). Modulatory connections in a thalamocortical pathway (cortical L6 to thalamus and thalamus to cortical L4) both have branches to TRN. In addition, both of them are connected to their counterparts in the other hemisphere. After suppressing pulvinar in one hemisphere, ipsilesional TRN cells are more active (Jones, 2009) which cause also suppression of the contralesional TRN and therefore disinhibition of contralesional pulvinar. This pulvinar excitation might influence the contralesional MIP activity by increasing its firing rate Fig 3.33. The fact that in ipsilesional MIP some cells show increase and some decrease might be due to the location of the cell in a specific cortical layer. For example it has 
been shown that cells in layer IV or V receive homotopic input from the contralateral hemisphere (Aboitiz, 1992; Pandya \& Vignolo, 1969; Seltzer \& Pandya, 1983), therefore overactivation of cells in contralesional MIP might also excite group of cells in ipsilesional hemisphere. In addition, inactivation of pulvinar might have direct down-modulation effect of ipsilesional MIP cells. This decrease of firing rate was expected and has been shown in other studies in monkeys (Zhou et al., 2016) and rats (Mohajerani et al., 2011). (Zhou et al., 2016) reported increased in the baseline firing rate of visual area V4 after ventrolateral pulvinar inactivation although the peak firing and attentional modulation after stimulus onset decreased after inactivation. In another study, inactivation of lateral pulvinar in Cebus monkeys caused both increased and decreased on responses in visual area V2, similar to our results (Soares et al., 2004).

a) pre injection (intact)

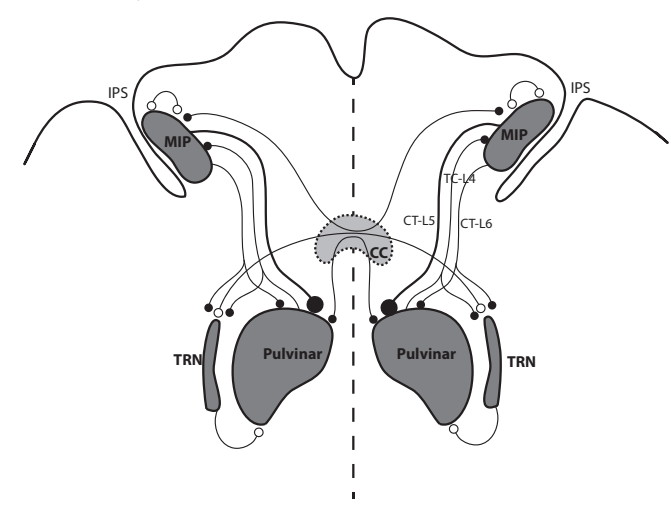

b) post injection

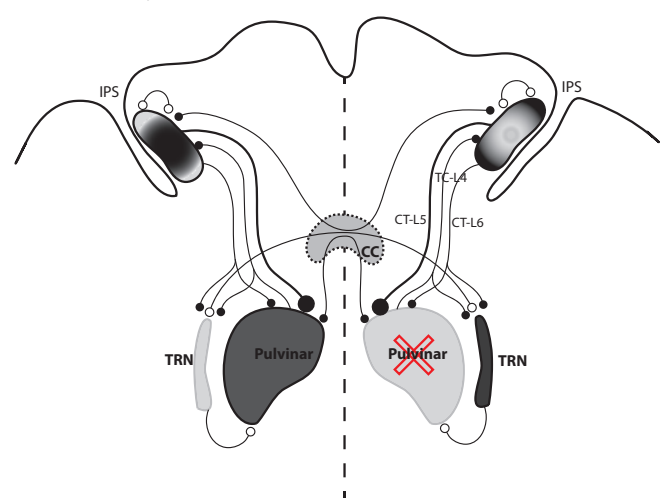

Fig. 3.33 Thalamocortical network of arm movement before (a) and after (b) pulvinar inactivation. (a) Before inactivation different regions are in their normal activity state (grayish color). (b) Thalamic reticular nucleus (TRN) input is the major inhibitory input to thalamus. Several minutes after inactivating right pulvinar, right TRN cells will be more active which it causes less activity in left TRN and consequently disinhibition of left pulvinar. This overexcitation of pulvinar cells in contralesional hemisphere might be the main cause of overexcitation of MIP cells during reach behavior that we observed. Moreover the excitatory input of MIP cells in left hemisphere to right hemisphere might be a source of excitation in a population of cells in right MIP (injected hemisphere). Of course the inhibitory influences inside cortex between cells across layers might be another source which causes suppression of activity in a population of cells.

TRN: Thalamic reticular nucleus; TC-L4: Thalamocortical connection to layer 4; CT-L5: Corticothalamic connection to layer 5; CT-L6: Corticothalamic connection to layer 6; CC: Corpus Callosum; MIP: Medial IntraParietal; IPS: IntraParietal Sulcus.

Note that connections with empty circle are inhibitory and with filled circle are excitatory and thicker connection means feedforward driving input. See (Grieve et al., 2000; Guillery \& Sherman, 2002; Jones, 2009; Sherman, 2017; Sherman \& Guillery, 2002)

But interestingly, the effects we observed in the area LIP (data not shown) were different. It is important to mention again that monkey performed dissociated saccade task in this part. Therefore, the direct comparison of these results with the results from MIP recording is only partially valid. We know that LIP and MIP both have reciprocal connections with dorsal pulvinar (Cappe et al., 2007; Hardy \& Lynch, 1992) but involved in a different frontoparietal network for goal-directed behavior (Andersen \& Cui, 2009). Although both of those areas in PPC are responsive to directing gaze and arm to spatially located targets but it has been shown that LIP is more active for eye-movement behavior than MIP for arm-movement (Snyder et al., 1997) and inactivation of them affects behavior in an effector-specific manner (Hwang et al., 2012; Kubanek et al., 2015). As a consequence, we 
decided to let the monkey perform a task which is more relevant to the recorded parietal area. In that respect we did not expect to see completely similar neuronal effects, after unilateral pulvinar inactivation, in area LIP and MIP.

We observed in both hemispheres that the mean modulation index for the population of LIP units were not significantly different from zero. This was consistent across task conditions and epochs. That means in both hemispheres and in different epoch, many units showed up- and many showed down-modulation but at the population level there was no effect.

Of course this analysis does not tell anything about the changes in the tuning of the units. From the current analysis we see mostly a general modulation of the firing rates which seems to be less dependent on the hand- or spatial tuning and is also less epoch-specific. Next we will investigate the effects on the units with specific task-response. One question is whether there is a correlation between modulation level and the tuning of the unit. Did the inactivation modulation level differ between group of units with certain type of tuning (e.g. contralateral hand tuning) and whether the direction of certain tuning changed after inactivation and this directional change depends on the modulation level. Another question would be whether pulvinar has different causal impact on parietal populations based on the cell types for example on visuomotor- or motor-like units in MIP (Hwang \& Andersen, 2011).

\subsubsection{Pulvinar inactivation modulates low frequency oscillations in MIP and LIP in a task-specific manner}

Unilateral pulvinar inactivation caused not only changes in the spiking activity of MIP units but also affected LFP oscillations in a frequency dependent manner, differently in opposite hemispheres. In the inactivated hemisphere, power in the lower frequency bands increased significantly almost independent of time (generally through the whole trial including fixation but not during movement execution). Similar effect has been observed in rat cortical area responsive to taste. When (Samuelsen et al., 2013) inactivated ventroposteromedial nucleus of thalamus (rat gustatory thalamus), power of LFP slow oscillations in the 1-9Hz band increased significantly (see also (Neske, 2016; Poulet et al., 2012)). Also in a recent study in monkeys, (Zhou et al., 2016), inactivated pulvinar and recorded from visual areas V4 and IT. The authors argued that inputs from the pulvinar may maintain cortical areas in an attentive and alert state and therefore this is necessary for the cognitive functions. Consistent with this, we observed that 2-3 hours after drug injection monkey became sleepy and tend to be demotivated to continue working.

But interestingly, in the intact hemisphere, overall power in lower frequencies slightly decreased after inactivation. Since similar effect was observed in area LIP, this might be considered as a global cortical effect but not area-dependent or even task-dependent effect. This effect can be explained by the excitatory-inhibitory interaction of thalamic communications. Thalamic relay cells are active in either tonic or burst firing modes suggesting that they not simply relay information to cortex but instead have modulatory influence on thalamocortical cells (Saalmann \& Kastner, 2011). For example, a relay cell transfers sensory information more efficient in awake than sleepy mode. When the brain is in an active mode, inhibitory cells in reticular nucleus of thalamus are least effective, thalamic relay neurons which project to cortex send signals in a most informative state. Instead in less attentive states or drowsiness, reticular cells are active at peak and relay cells are firing bursts in a slow frequency 
rhythms which causes high amplitude slow waves of delta to alpha frequencies (2-15 Hz) (Jones, 2009).

Main frequency component of the parietal LFP activity is in beta band (13-30Hz) (Dean et al., 2012; Hwang \& Andersen, 2011; Lehmann \& Scherberger, 2015; Pesaran et al., 2008; Scherberger et al., 2005; Stetson \& Andersen, 2014). This frequency band encodes spatial preference (Dean et al., 2012; Scherberger et al., 2005) as well as arm-preference (our data). From previous evidence beta band power is associated with a steady state behavior. That means beta band is usually higher when there is no active movement planning is under process but the subject is waiting for commanding information to plan a movement accordingly. For example (Stetson \& Andersen, 2014) showed that beta power is higher during fixation than memory (planning) period and is lowest during movement execution. In addition, decrease of beta power is associated with the tuning of recorded site (Dean et al., 2012; Hwang \& Andersen, 2011, 2012; Rossiter et al., 2014). Consistent with this, high level of beta band activity was observed in Parkinson disease which have rigidity or slowness in their movements and pathological treatments which target dopaminergic system lowers the beta level and improves the behavioral state (George et al., 2013; Little \& Brown, 2014).

It has been shown that higher order thalamus modulates cortical oscillations in theta, alpha, beta and gamma frequencies in different cognitive processes such as memory and attention (Ketz et al., 2015; Saalmann et al., 2012; Schmid et al., 2012; Sweeney-Reed et al., 2014; Zhou et al., 2016). But the functional role of pulvinar oscillatory activity in reach behavior is still unclear. Reciprocal connections between pulvinar and frontoparietal network for reaching (Battaglia-Mayer et al., 2016; Caminiti et al., 2015; Grieve et al., 2000) suggests similarities in oscillatory component but whether oscillations in pulvinar are just mirror of cortical inputs or they play a functional role should be still elucidated. In the previous chapter we have shown that beta band frequency has a peak power in reach and saccade behavior and modulates during the task, while theta and gamma also slightly respond to certain task conditions. In this study we address the question whether pulvinar plays a functional role in oscillatory activity in parietal cortex.

We did observe different effects in low and high beta band power in both hemispheres, as if they contribute to different functional process. In the inactivated hemisphere, lower beta band did not change significantly (but increased slightly) when contralesional arm was used but decreased significantly when ipsilesional arm was used. But in opposite, higher beta increased for contralesional trials and decreased for ipsilesional trials (stronger for ipsilesional space). Interestingly the beta band power at the population level shows that power for reach with ipsilesional arm to ipsilesional target is lower than reaches to contralesional target. In addition, the beta band power (especially high beta) for contralesional arm relative to ipsilesional arm increased after inactivation.

Given that decrease of beta in delay and pre-movement periods is associated with reach planning process, our data shows that decrease of beta oscillations for ipsilesional and increase for contralesional arm movement after pulvinar inactivation is consistent with the behavioral effects (slowness and unwillingness of contralesional arm movements). In addition, interestingly the decrease of beta power with ipsilesional hand was stronger for ipsilesional than contralesional targets which is consistent with the spatial effect after inactivation. But the effects in the opposite hemisphere were different. Lower beta power decreased in all conditions but more for contralesional than ipsilesional arm and 
high beta just increased in all conditions similarly. This effect might be because intact hemisphere was released from inhibition and consequently a compensatory effect that intact hemisphere takes.

Another effect which was dramatically different across hemispheres was the gamma band activity. While in the inactivated hemisphere, gamma band (mostly low gamma) just slightly increased (more for contralesional targets, but mostly not significant,), in the intact hemisphere gamma band power in all conditions and time-independent significantly increased. This increase was just slightly more for ipsilesional space during movement. This could be because of the increased firing rate in intact hemisphere which could leakage to LFP power (Ray \& Maunsell, 2011; Waldert et al., 2013). As we mentioned before most of the units in the intact hemisphere were firing more after inactivation in an epoch non-specific manner and that could lead to increased power of high frequency LFP.

\subsubsection{Pulvinar inactivation modulates LIP activity for saccades differently than MIP activity for reaches}

Interestingly we observed similarities as well as differences compared to the experiment in MIP. After unilateral pulvinar inactivation LFP power in area LIP in the inactivated hemisphere increased significantly in a time-independent manner in lower frequencies, as we observed in area MIP. But in LIP unlike MIP, beta band power in all conditions (irrespective of hand and space) decreased during fixation and delay periods although stronger for the ipsilesional hand. Similar to MIP, gamma band power did not change significantly after inactivation.

But interestingly, in the intact hemisphere, we observed a decrease of power in the lower frequency bands (stronger when monkey fixated with contralesional hand). Alpha and lower beta bands during fixation and cue period decreased in all conditions, stronger for contralesional hand. One of the main differences is that higher beta and gamma band power, unlike in MIP, did not change dramatically after inactivation but it changed epoch and space-specific. In LIP, gamma band power during delay increased significantly for ipsilesional targets more than contralesional targets which supports the idea that intact hemisphere overrepresents the ipsilesional space because of disturbance in the push-pull mechanism between two hemispheres.

\subsubsection{Summary}

In summary, this study tries to shed light on the causal role of dorsal pulvinar in reach and saccade neuronal responses in parietal areas. We showed that pulvinar unilateral inactivation caused mainly contralesional hand/space behavioral deficits. We also showed that spiking activity in parietal area MIP was affected differently in opposite hemispheres while monkey performed a reach task. At the population level, while firing of most MIP units in the intact hemisphere increased after pulvinar inactivation, in the inactivated hemisphere both decrease and increase of firing were observed.

In addition, there are more specific questions which needs to be clarified and more investigated. One question is how effector-specific is the changes in the firing rates after pulvinar inactivation. That means to check whether the changes in firing rate across task epochs remain more or less in the range of changes in the fixation period or it varies depending on the level of visuomotor processing. The second question which is still under investigation is whether and how pulvinar inactivation changes 
hand and space tuning in parietal cortex. More specifically, we hypothesize that pulvinar inactivation might changes the spatial and hand preference of neurons in the direction of behavioral effects.

Pulvinar inactivation caused strong and consistent (across sites and sessions) changes in the LFP power in MIP, both in the inactivated and the opposite hemisphere. While low frequency power (delta-theta-alpha) in the inactivated hemisphere significantly increased (in the entire trial except movement), in the intact hemisphere higher beta and gamma band power increased. Interestingly beta band power in the inactivated hemisphere increased for the contralesional and decreased for the ipsilesional hand. In the intact hemisphere, beta decreased strongly for contralesional hand. Strongest beta increase in delay was for contralesional space and hand (and vice versa). These diverse neuronal changes might reflect functional impairment and compensatory mechanisms enable the monkey to successfully perform the tasks even after the inactivation.

A proper motor planning and execution require an interaction within and between neuronal populations with efficient timing in terms of neuronal oscillations (Fries, 2005). Therefore, an efficient behavioral output would be dependent on an efficient neuronal communication with proper timings. We expected that dPul inactivation as a perturbation in the goal-directed network, changes intra- and inter-areal neuronal communications therefore we measures the changes in spikes and local field potential neuronal synchronization within and across hemispheres (Fries, 2005; Purushothaman et al., 2012; Shumikhina \& Molotchnikoff, 1999; Sweeney-Reed et al., 2014; Zhou et al., 2016). As the next step, we aim to address this by measuring field-field as well as spike-field synchrony in the MIP populations before and after pulvinar inactivation. We hypothesize that pulvinar disruption might effect synchronization in the beta band as well as theta band power mostly during contralesional behavioral planning and movement.

Although our results seem to be consistent across sessions but we require to perform more experiments with recording from LIP to be more conclusive about our findings. In addition, to confirm that observed effects are related to deactivate pulvinar (due to THIP injections), we need to perform the same experiment while injecting a neutral solution such as saline. And finally we require to redo the experiment with a second monkey to hopefully replicate the results.

Altogether our results show that pulvinar is important for visually-guided arm and eye movements. Pulvinar seems to causally mediate neuronal activity in parietal cortex in a task- and limb-specific manner differently across two hemispheres such that disruption of pulvinar causes severe behavioral deficits. These diverse push-pull changes might reflect both induced functional impairment and compensatory mechanisms that enable the monkey to successfully perform the tasks even after the inactivation.

\subsection{Acknowledgments}

I especially acknowledge Dr. Kagan for his unlimited support during this project. I thank Lukas Schneider for programming task controller and preprocessing of the behavioral as well as electrophysiological data and great scientific discussions and also Benjamin Dann and Swathi Sheshadri for their support in LFP analysis. I also thank Mathieu Pachoud and Esther Akinola for help with the spike sorting process. We appreciate Daniela Lazzarini, Ira Panolias, Sina Plümer, Leonore Burchardt, Klaus Heisig, and Dirk Prüße and Ralf Brockhausen for technical support. 



\title{
Chapter 4
}

\section{Changing saccade plans: response competition in spatial decision-making}

\begin{abstract}
4.1 Abstract
Animals and humans can rapidly change goals and actions according to updated demands of the environment. This flexible action selection is thought to be mediated by sensorimotor circuitry through competition of neuronal signals representing different response options ("affordance competition hypothesis", (Cisek, 2007)). The aim of this study was to understand the behavioral manifestations of multiple response options in saccadic internally-driven choices (so called "free-choice"), and how updating the saccade goals at different intervals during motor planning modifies the selection of spatially lateralized options in instructed or free-choice conditions. Sixteen human subjects participated in two sessions and performed three different oculomotor tasks in blocks: 1) Primary saccade task, 2) Follow Second Cue (FSC) task, and 3) Follow First Cue (FFC) task. The primary task included only one instructed or free-choice central symbolic cue ("go" signal), which prompted subjects to perform speeded saccade to left or right hemifield. In FSC and FFC task 66\% and 50\% trials were as in the primary task, but in the rest of trials the cue changed following a variable delay after the first cue ("change trials", change signal delay, CSD: 17, 67, 134, 184, 251, $301 \mathrm{~ms}$ ). In the FSC task in change trials, subjects were required to attempt updating their action and also to report the direction of their initial motor plan (left or right) with a button press. This allowed us to distinguish "real" change trials from "congruent" trials in which the second cue directed the subjects in the same direction as their initial plan (as could be the case in a large proportion of choice trials). In the FFC task subjects always had to follow the initial cue and ignore the second cue. This task served as a control to demonstrate that subjects perceived the first cue when it was followed by the second cue. We hypothesized that in the choice trials, two alternative motor plans are initially present and it takes longer to resolve the competition and arrive at decision threshold, as compared to the single response option instructed trials. In accord with this hypothesis, in all three tasks in no change (primary) trials subjects were significantly faster in the instructed as compared to the choice condition. In the Follow Second Cue task the change reaction times (measured from the second cue) were faster than in primary trials, significant for the choice trials. This suggests that it is easier to switch between actions during motor planning than to initiate the action from a fixation baseline, especially when both action plans might be engaged. Correspondingly, subjects had better performance in the choice change trials as compared to the instructed change trials, at early and middle CSDs (significant at 134 and $184 \mathrm{~ms}$ ), but not at late CSDs. The (task-irrelevant) second cue also biased the choice target selection when the second cue appeared within $134 \mathrm{~ms}$. Together, these results support the hypothesis that the competing oculomotor plans are present in the interval up to $200 \mathrm{~ms}$ leading to the saccade execution.
\end{abstract}




\subsection{Introduction}

Survival in ever-changing environment requires flexibility in action selection (Cisek, 2012; Cisek $\&$ Kalaska, 2010). This includes not only being fast and accurate enough in preparation of a single action but also readiness to stop or update the action during planning or execution. Sometimes, actions are instructed or "forced" based on the current contextual rules, e.g. green traffic light instructs you to drive the car. But often actions are an outcome of a decision between several available choices, e.g. drink water, juice or tea when you are thirsty.

Subjective value or utility of each option plays a crucial role in these decisions. For example, associating an external contingency such as higher reward or lower effort to a certain option biases decisions toward this option, comprising value-based decisions (Kubanek \& Snyder, 2015a; PadoaSchioppa, 2011; Rangel \& Clithero, 2012). But in the absence of such clearly defined external variables, decisions have to be guided by internal variables, e.g. subjective preferences, strategies based on memories of past choices and outcomes, as well as noise fluctuations in the underlying neural circuitry (Funahashi, 2017; Mochizuki \& Funahashi, 2014, 2016). Such internally-guided decisions are often called "free-choice" or volition (Christopoulos et al., 2015b, 2018; Coe et al., 2002; Dominguez-Vargas et al., 2017; Fleming et al., 2009; Gorea et al., 2014; Marcos \& Genovesio, 2016; Nachev et al., 2005; Suriya-Arunroj \& Gail, 2015; Wardak et al., 2002; Wilke et al., 2012, 2013, 2018, 2010), although the value-based choices between clearly distinct, explicitly signaled options (e.g. risky vs. certain lotteries) are also termed "free choice" (Grabenhorst et al., 2012; Yamada et al., 2018). While significant progress in understanding value-based decisions has been made (Schultz, 2015), the behavioral and underlying neural mechanisms of free choice are not fully understood (Fitzpatrick et al., 2018). Causal interference studies in macaque monkeys used reversible inactivation or stimulation in tasks that included free choice to understand the contribution of certain brain regions in decision making (Christopoulos et al., 2015b; Dominguez-Vargas et al., 2017; Wardak et al., 2002; Wilke et al., 2012, 2013, 2010). It is still not clear however how the brain resolves the competition or conflict between options of similar utilities and reaches the decision threshold.

Furthermore, flexible behavior relies not only on selecting and performing a single predetermined action, but also on adaptively changing action plans depending on what the environment demands at the moment. It might be stopping a movement preparation or an ongoing action (Aron et al., 2014; Boecker et al., 2013; D. Logan \& B. Cowan, 1984; Verbruggen \& Logan, 2008b), changing from one action to another, e.g. switching between responses with different hands or fingers (Boecker et al., 2013; Fleming et al., 2009; Obeso et al., 2013), or changing only the properties of already initiated action, such as direction or amplitude during online control of hand movements (Archambault et al., 2009, 2011)or changing saccade direction in midflight (Ray et al., 2004). Several task designs have been used to study response inhibition and response reprogramming, most prominently the stop-signal paradigm (D. Logan \& B. Cowan, 1984), but also double-step task (Becker \& Jürgens, 1979), redirect double step task (Camalier et al., 2007; Ray et al., 2004), stop-change task (Logan \& Burkell, 1986; Obeso et al., 2013; Verbruggen \& Logan, 2008b) and search-step task (Camalier et al., 2007). Boecker and colleagues (Boecker et al., 2013) extensively reviewed the literature on stop-signal and stop-change studies. Some recent work combined these tasks with other methods such as TMS (Hartwigsen et al., 2012; Obeso et al., 2013), lesions (Gremel \& Costa, 2013; Nachev 
et al., 2007), fMRI (Boecker et al., 2011; Mars et al., 2007; Nachev et al., 2005), EEG (Fleming et al., 2009) or MEG (Jha et al., 2015) to address functional aspects of brain processing related to action stop or change behavior. Since in change tasks the initial action should be stopped or modified, some of these studies investigated whether same general inhibition process works in both tasks or not. The current view suggests separate processes for response inhibition and switching (Camalier et al., 2007; Verbruggen \& Logan, 2008b).

While behavioral aspects and neural mechanisms of response inhibition have been investigated extensively, updating response to a new relevant option was not as much studied, especially in the oculomotor system (Archambault et al., 2011; Ramakrishnan et al., 2012). Therefore, in the present study we focused on three interesting aspects of human saccade action selection: free choice target selection, changing saccade plans during instructed or free choice, and inhibition of invalid or irrelevant change signals during the same conditions. The overall aim of this study was to understand how updating the goal at different intervals during motor planning modulates the selection of spatially lateralized options in instructed or choice conditions.

To this end, we used a modified version of stop-signal paradigm in which human subjects were required to do a speeded saccade toward one of two (bilaterally presented) targets, either instructed or freely chosen, as indicated by a central symbolic cue. In a subset of trials the appearance of a second cue with a short variable delay after the first cue prompted subjects to change their plan (if they already formed one) and make a saccade toward the other target. This task gave us the opportunity to study the behavioral difference of saccades during internally-driven choice and externally-driven instructed contexts, without or with an interference of new information.

Our approach has been largely motivated by influential work of Cisek and colleagues on understanding action selection and decision making (Cisek, 2007; Cisek \& Kalaska, 2010). This view proposes that the action selection and action specification occurs at the same time and in the same interactive, recurrent neural circuitry (parallel processing or affordance competition hypothesis). In this framework, several potential response options are represented through multiple neural populations at several stages of sensorimotor processing and are in a continuous interaction or competition; while other signals representing the relevant contextual information (e.g. rules, expected reward, effort) are biasing this interaction toward the eventual action selection and implementation (Christopoulos et al., 2015a). We hypothesized that in choice trials two alternative neural representation of possible spatial targets develop after the presentation of the choice cue. Those representations compete with each other and the resolution of this competition leads to slower reaction times in choice trials as compared to instructed trial where only one saccade goal is externally cued. Following the same reasoning, we predicted that changing to an opposite response option in choice trials would be faster and easier than in instructed trials. In other words if two representations are initially active during the planning and a new instruction requests changing the plan to the opposite side, the switch of action will be more readily made because the trace of an alternative (and now valid) plan still existed. 


\subsection{Methods}

\subsubsection{Participants}

Subjects were recruited via the online platform "Studentische Jobbörse Göttingen" of the University of Goettingen (http://www.uni-goettingen.de/de/jobangebote/29718.html). Sixteen healthy volunteers participated in our study ( 8 females; age (MEAN \pm SD): $25.4 \pm 5.1$ ). Four participants ( 1 female; age 22.3 \pm 5.3 ) were excluded from final analysis due to specific performance criteria (see section on Subjects exclusion criteria). We used the data of the twelve remaining subjects in our analysis (7 females, age 26.4 \pm 4.9 ). The experimental procedures were approved by the research ethics committee of the Georg-Elias-Müller-Institut für Psychologie at the University of Göttingen. All subjects were financially compensated for participation with 8 Euro per hour. They had normal or corrected-to-normal vision and they were initially naive to the tasks.

\subsubsection{Apparatus and data acquisition}

The tasks were designed with Matlab (Mathworks) version 2011b using the Psychophysics toolbox version 3.0.10. Subjects were seated in front of a monitor (Dell 2007FP Flat Panel LCD Monitor, 20.1 " diagonal, $1600 \times 1200$ resolution at $60 \mathrm{~Hz}$ ) at $50 \mathrm{~cm}$ viewing distance. The head was stabilized using a chinrest and a forehead holder. The height of the chinrest was set so that the subjects' eyes were approximately at level corresponding to the center of the monitor. Eye position was recorded using a miniature monocular infrared camera (mounted on a safety glasses frame), with a sampling rate of $60 \mathrm{~Hz}$ (Viewpoint eye-tracker software, Arrington Research, version 2.9.2.5 USB-60x3). We calibrated the eye position using routine calibration procedure of the Viewpoint software.

\subsubsection{Experimental procedures and tasks}

Our aim was to study the behavioral aspects of competition between saccade plans. More specifically we wanted to understand whether and how unexpected relevant and also irrelevant changes affect the saccade target selection in different contexts: when a human subject is instructed or is free to select between two potential targets. Our experimental paradigm was a modified version of the stop-signal paradigm (D. Logan \& B. Cowan, 1984; Verbruggen \& Logan, 2008b) mostly used to study response inhibition in goal-directed behavior. In the stop-signal paradigm subjects are cued to perform an action and in a subset of trials to stop the planned action. In the present study we used a similar structure to investigate the behavioral aspects of changing saccade plans. Each subject participated in two sessions on separate days. In each session they performed three different tasks: Primary task (PRM), Follow Second Cue (FSC) task and Follow First Cue (FFC) task. Before the actual data collection, participants were informed about the task details by a written instruction and performed a practice block of 50-100 trials. Each session started with the Primary task followed by the other two tasks interchangeably. Fig. 4.1(A) shows the order of the tasks, approximate number of trials and the time spent in each block. The order of the FFC and FSC tasks was changed between sessions to avoid sequence effects. There was a break of 20 minutes between the FFC and FSC tasks and 3-5 minutes between blocks of the same task. Subjects responded with a saccade toward one of the bilaterally presented targets according to the requirements of the presented cue. Participants were instructed 
to respond as fast as possible while trying to be correct as well (the emphasis was on the speed of responses).

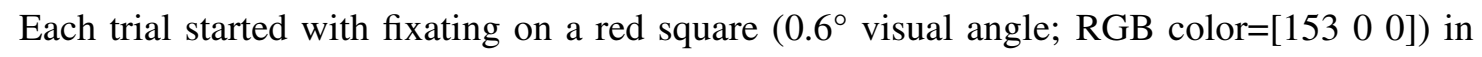
the middle of the screen. After a variable delay $(500-1300 \mathrm{~ms})$ a cue replaced the fixation square simultaneously with two bilateral blue target squares (each $0.6^{\circ}$ size, placed at $10^{\circ}$ eccentricity in the left and right; RGB color= [128 128 255]). Both targets were placed in the same horizontal level, but they were presented either at the same level or at $20^{\circ}$ angle above or below the central fixation (6 positions in total). The presented cue could be an instructed left cue (leftward arrow), instructed right cue (rightward arrow) or a free-choice cue (equality sign). The size of the instructed cues was $1.3^{\circ} \mathrm{X}$ $2.6^{\circ}$; choice cue $1.5^{\circ} \times 1.5^{\circ}$ (RGB color=[255 255255$]$ ). These symbolic cues served as the "go" cue and subjects were required to do the eye movement as soon as one cue appeared on the screen. In a subset of trials in the FSC and FFC tasks, the cue was replaced with another cue after a short delay. A target was selected when the eye position entered a circular window ( $6^{\circ}$ radius) around that target. Subjects had $1000 \mathrm{~ms}$ to select a target. If they did not select any target within this time, the trial was aborted and the message "too late" was presented on the screen. In each trial after subject selected a target, a white circle $\left(0.5^{\circ}\right.$ radius; $\left.\mathrm{RGB}=\left[\begin{array}{l}255255255\end{array}\right]\right)$ appeared around that target for $200 \mathrm{~ms}$, as a confirmation. Subjects did not receive any specific feedback whether they selected the correct or the incorrect target. All features were presented on a gray background $\left(\mathrm{RGB}=\left[\begin{array}{l}51 \\ 51\end{array}\right.\right.$ 51]). Different trial types of each task were pseudo-randomly interleaved in each block. For each block we made the full list of all possible trial types with the proportional number of trials for each trial type. Then in each trial we selected randomly from the list.

The Primary task contained only single cue "primary trials" (Fig. 4.1(B), left panel), i.e. only instructed (instructed-left or instructed-right) and choice (free choice) trials. In the instructed trials, subjects had to saccade toward the target that the cue pointed to and in the choice trials, they were free to choose any of the saccade targets. In each session participants performed one block of this task including about 240 trials ( $~ 50 \%$ instructed: $\sim 25 \%$ instructed left and $\sim 25 \%$ instructed right, and $\sim 50 \%$ choice).

In the Follow Second Cue (FSC) task (Fig. 4.1(B), middle panel), $\sim 67 \%$ of the trials were primary trials (same as in the primary task) and the other $\sim 34 \%$ were "change trials", i.e. choice change $(\sim 17 \%)$ and instructed change trials $(\sim 17 \%)$. In change trials the first cue (which could be instructed or choice) stayed on the screen for a certain time (change signal delay "CSD") and then was replaced by 2 nd instructed cue. To be able to probe the dynamics of flexible saccade target selection, we used six different CSDs for each change trial type (multiples of the screen refresh rate at $60 \mathrm{~Hz}$ ): 17, 67 , 134, 184, 251 and $301 \mathrm{~ms}$. The FSC task contained two times more trials for intermediate CSDs $(134,184$ ad $251 \mathrm{~ms})$ than for other CSDs; i.e. 11, 11, 22, 22, 22, 11\% of trials in each change type respectively. 


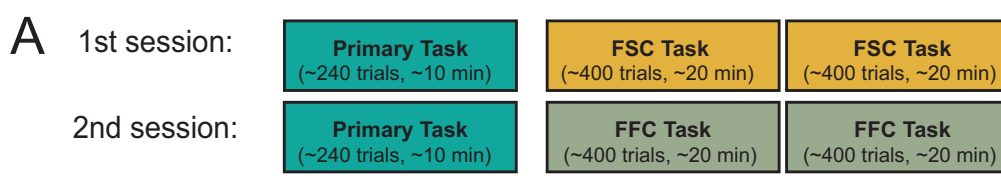

\begin{tabular}{|c|c|}
\hline $\begin{array}{c}\text { FFC Task } \\
(\sim 400 \text { trials, } \sim 20 \mathrm{~min})\end{array}$ & $\begin{array}{c}\text { FFC Task } \\
(\sim 400 \text { trials, } \sim 20 \mathrm{~min})\end{array}$ \\
\hline \begin{tabular}{c|c} 
FSC Task \\
$(\sim 400$ trials, $\sim 20 \mathrm{~min})$
\end{tabular} & $\begin{array}{c}\text { FSC Task } \\
(\sim 400 \text { trials, } \sim 20 \mathrm{~min})\end{array}$ \\
\hline
\end{tabular}

B

$\begin{array}{rr}\text { Primary task } & \text { Follow second cue (FSC) task } \\ \text { (34\% change trials) }\end{array}$

Follow first cue (FFC) task
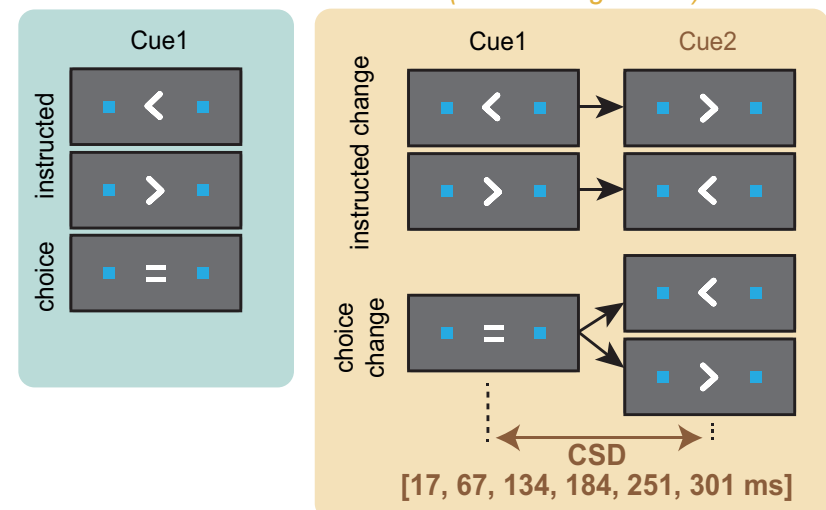

(50\% change trials)

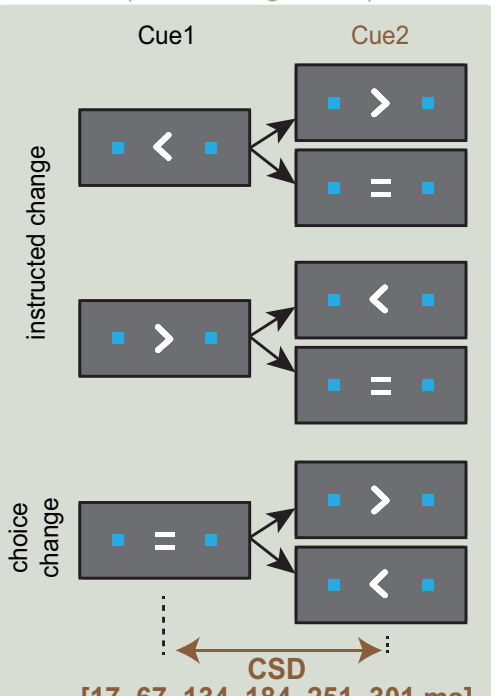

Example choice-to-instructed change trial (FSC task)

$[17,67,134,184,251,301 \mathrm{~ms}]$

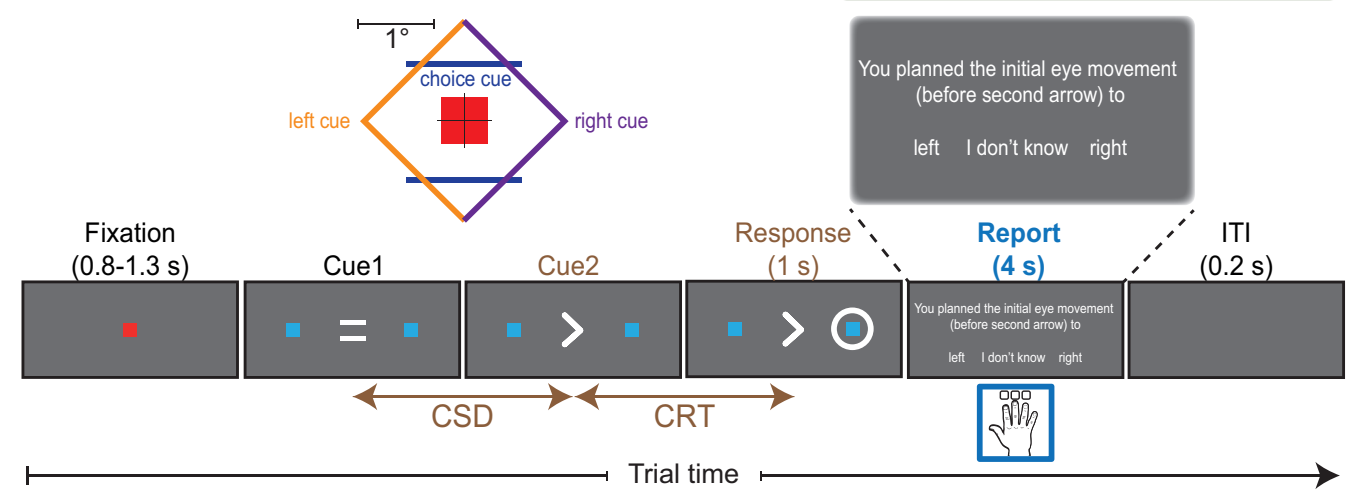

Fig. 4.1 Experimental design. (A) Sequence of tasks in two experimental sessions for each subject. Subjects participated in two sessions and performed three different tasks. In the beginning of each session they performed the Primary task. They also performed two blocks of FSC and FFC task in each session. The order was counterbalanced between two sessions. Between performing different tasks, subjects had a 20 minutes break and between blocks of the same tasks 5 minutes break. (B) Tasks description. Primary task (left panel), Follow Second Cue (FSC) task (middle panel) and Follow First Cue (FFC) task (right panel). All trials started with a random fixation period (500-1300 ms). Tasks included either only primary trials or primary and change trials. In primary trials, after the fixation a cue together with two targets in the periphery appeared on the screen. The cue was either an instructed (a leftward or rightward arrow: subject did saccade toward the relevant target) or a free choice (equality sign: subject freely chose saccade to one of the targets). The cue served also as a go signal and subjects had to do saccade as soon as possible after the cue appearance. In change trials, with a short delay (CSD) after the first cue, another cue appeared which subject required updating the plan toward the new relevant target. Primary task contained only primary trials. FSC task contained both primary (66\%) and change (34\%) trials. After change trials in this task, subjects had to report their initial motor plan with a button press. FFC task was similar to the FSC task but with 50\% primary and 50\% change trials. In this task subjects had to ignore the second cue and respond to what first cue required. Only in this task the second cue could be a choice cue. (C) Timeline of an example successful change trial in FSC task. After a short fixation, an instructed leftward cue appeared, asking subject to make a saccade choice between left or right target. After a short change signal delay (CSD) the first cue changes to the second cue (instructed right), instructing the subject to saccade toward the right target. In this example subject did the saccade to the right target correctly. The white circle shows the target selection, regardless of the correctness. Afterwards subject responded to a question ("you planned initially to do the eye movement to") by pressing a keyboard button with one of the fingers of right hand. Comparing the initial plan with the second cue enabled us to isolate real plan changes. In this example if subject reports the initial plan by pressing the left keyboard button we assign this trial as a correct "Real" change. The inset shows the spatial configurations of the fixation point and the cues $(>,<,=)$ 
In change trials, as soon as one of the targets was selected (no matter whether correct or incorrect) the following text was presented on the screen: "You planned the initial eye movement (before second arrow) to: left, I don't know, right". Subjects had to choose one of the options to indicate the target they initially planned for by pressing the "left arrow", "upward arrow", or "right arrow" buttons on the keyboard using the index, middle or ring finger of the right hand, respectively. They had up to 4 seconds to select an answer.

This retrospective report gave us the opportunity to classify the change trials to trials in which subjects changed their initial plan as "Real" change trials, trials in which they stayed with the initial plan as "Congruent" trials, and trials in which they were not aware or unsure of their initial plan as "I don't know" trials (see section Data analysis).

Fig. 4.1(C) illustrates the timeline of one successful trial in the FSC task. This example illustrates a choice change-to-right trial. The second (change) cue is presented after CSD time following the first cue. The subject initiates the eye movement toward the right target after change reaction time (CRT) following the second cue. After target selection, subject reports the initial saccade plan. In this trial a "Real" change would be if the subject reports the initial plan by pressing the "left" button and it would be a "Congruent" change (i.e. no actual change) if subject presses the "right" button.

The Follow First Cue (FFC, Fig. 4.1(B), right panel) control task was designed for two reasons. First, we used it to assess the potential effect of perceptual masking in change trials. In choice change trials of FSC task the second instructed cue was presented in the location partially overlapping with the preceding choice cue (Fig. 4.1(C), inset). Although the shapes of the cues were very different and the spatial overlap minimal, we decided to investigate whether this overlap could affect the visibility, especially in fast change trials (short CSDs), e.g. by masking the initial choice cue. To be able to check the masking effect, we added a new condition in this task in which the first cue was an instructed and the second was a choice cue (instructed-to-choice change trials; Fig. 4.1(B) middle panel). Then we compared the performance in these trials versus the instructed-to-instructed change trials. In instructed change trials, the two instructed cues were not overlapping: their locations were next to each other.

The second reason for the FFC task was to investigate how task-irrelevant changes in the environment modify subjects' behavior. While in the main task (FSC) subjects were explicitly asked to update their saccade goal when a cue changes, in the FFC task we instructed them to maintain their initial goal and ignore the distracting (task-irrelevant) second cue. In this task unlike the FSC task subjects were not required to report their initial plan since no changing was asked. This task contained $\sim 50 \%$ change trials with nearly equal number of trials for each change trial type (instructed-instructed, instructed-choice, choice-instructed). There was nearly equal number of trials for each CSD in each change trial type.

\subsubsection{Data analysis}

Saccades were detected when the eye movement velocity exceeded above the threshold of 30 degrees/s and remained over this threshold for minimum $30 \mathrm{~ms}$. We did not consider saccades starting earlier than $100 \mathrm{~ms}$ after the first cue or $50 \mathrm{~ms}$ after the second cue in change trials for further analysis because previous studies have shown that the visual response has a minimum latency of 50-100 ms in the oculomotor system (Gold \& Shadlen, 2000; Pouget, 2005), and we wanted to make sure that 
subjects could at least in principle react to the cue stimuli (even if they were not able to change successfully, resulting in incorrect change trials). We compared reaction times (RT), free choice preference and performance (success rate) across different task conditions. RT in primary trials was defined as the latency between the appearance of the (first) cue and the initiation of saccade. The change reaction time (CRT) was defined as the latency from the appearance of the second cue to the saccade initiation.

Choice preference was defined as the proportion of selecting one target (left or right) to the total completed primary choice trials. Consequently we had right-choice-preference and left-choicepreference, complementary adding to $100 \%$. The other aspect was the performance or success rate in each trial type. Success rate for each trial type was defined as the number of correct responses divided by the number of all completed trials in that trial type. We calculated success rate for primary instructed trials of all tasks, choice-instructed and instructed-instructed change trials of the FSC task and instructed-instructed and instructed-choice change trial of the FFC task. As expected, subjects were successful in nearly all primary instructed trials: the success rate in primary instructed trials was on average $98.9 \pm 0.1 \%$ (mean \pm SEM) in the Primary task, $98.6 \pm 0.7 \%$ in the FSC task and $98.1 \pm 0.5 \%$ in the FFC task. In the Results section we report in detail the success rate in choice and instructed change trials of FFC and FSC tasks.

Categorization of reports in FSC change trials As it was mentioned before, in the FSC task subjects required to report their initial saccade plans (before the second cue). This helped us to isolate, according to subjects' reports, the trials in which "Real" changes of plans happened from the "Congruent" trials (no actual change) and also from those in which subjects were not aware of or did not remember the initial plan ("I don't know"). On average, the percentage of "Real", "I don't know" and "Congruent" trials were $87.8 \pm 3.7$ (mean \pm SEM), $4.4 \pm 1.6$, and $7.8 \pm 2.5$ in instructed change trials and 37.1 $\pm 3.6,20.7 \pm 4.9,42.3 \pm 2.8$ in choice change trials (Fig. 4.2(A)). In instructed-instructed change trials participants reported most of the trials as "Real" changes $(87.8 \pm 3.7 \%)$ but in choice-instructed change trials only less than half $(37.1 \pm 3.6)$. Subjects could successfully change the saccade plan toward the correct target in $46.8 \pm 6.6 \%$ of instructed-instructed and in $22.8 \pm 3.6 \%$ of choice-instructed change trials.

In choice-instructed trials subjects reported more "I don't know" than in the instructed-instructed condition $(20.7 \pm 4.9 \%$ versus $7.8 \pm 2.5 \%)$. We expected to see this because decision after the choice cue takes longer than a plan after an instructed cue and therefore in choice change trials and especially when the CSD is short subjects might have no clear plan yet before the second cue appears. Our data shows that the percentage of "I don't know" reports decreases as the CSD increases (data not shown).

While in instructed change trials subjects reported only less than $10 \%$ "Congruent", in choice change trials they reported slightly more "Congruent" than "Real". Theoretically, for an ideal subject we expect to see 50\% "Real" and 50\% "Congruent" in choice-instructed trials, and 100\% "Real" reports in instructed-instructed change trials. In other words, no matter what the primary choice preference of the subject is, when we consider choice change trials (both choice-change-to-right and choice-change-to-left), in half of the trials the ideal subject should really change the plan and, while in the other half the plan from the beginning should be to saccade to the target which will be instructed with the second cue. 
It is also likely that in change trials with short CSDs subjects haven't yet formed a clear plan at the time of the second cue and report "I don't know" in some trials. On the contrary, in instructed change trials, an instructed first cue should trigger the relevant plan immediately and subjects should be aware of that plan. Therefore we expect to see only "Real" reports in this change trial type.
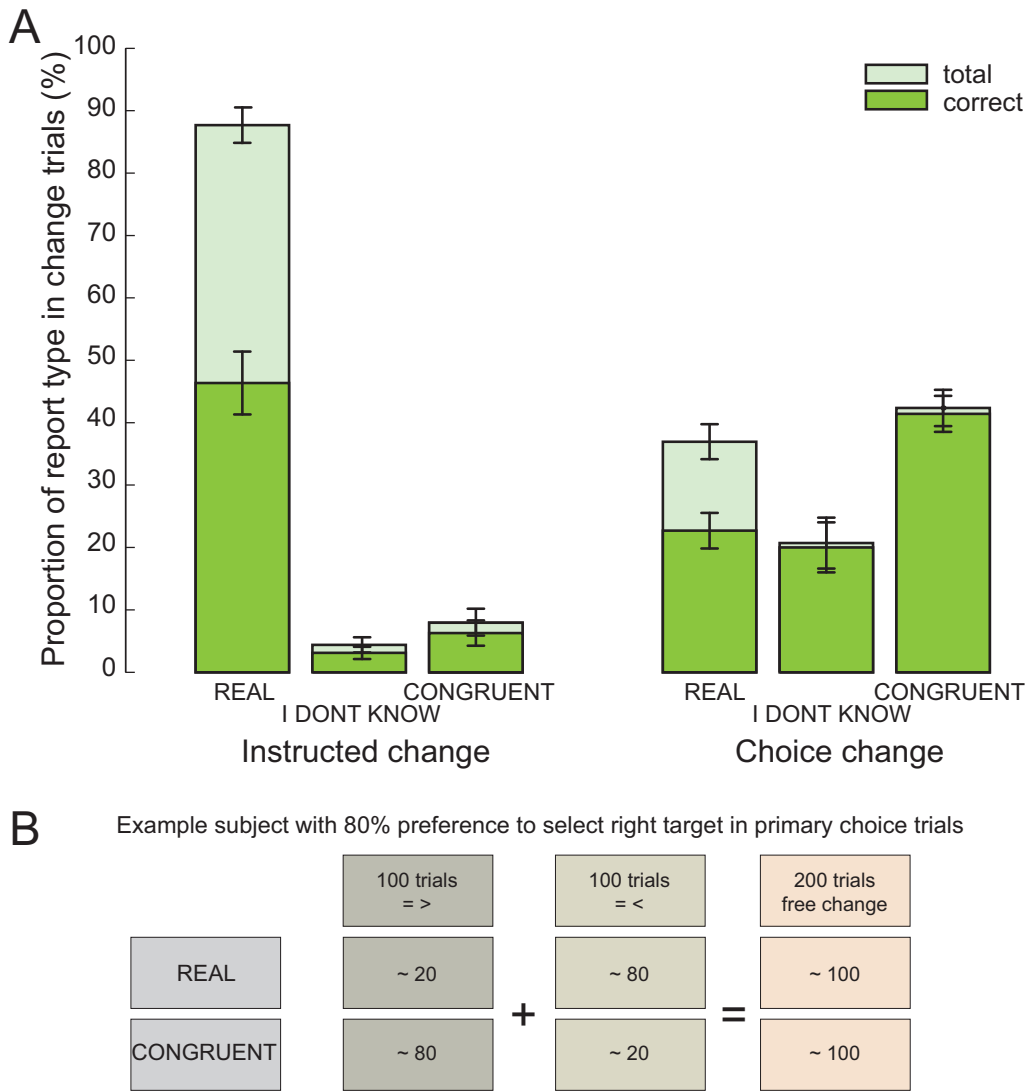

Fig. 4.2 Proportion of subjective reports in instructed and choice change trials in FSC task. Percentage of "Real", "I don't know" and "Congruent" reports in each trial type adds up to 100\%. Dark color shows the proportion of total trials and light color the proportion of correct trials. For example in choice change trials, subjects reported in $37.1 \pm 3.6 \%$ (MEAN \pm SEM) of trials "Real" changes and $22.8 \pm 3.6 \%$ were correct trials but they reported $42.3 \pm 2.8 \%$ as "Congruent" with $41.3 \pm 2.7 \%$ correct. B) An example that depicts why subjects in change trials of FSC task ideally should report 50\% "Real" and 50\% congruent regardless of their choice preference. Suppose a subject with $80 \%$ preference for selecting right target in primary choice trials. Therefore the initial plan in choice-instructed change trials should be plan for right target. Consequently we expect theoretically that this subject report $80 \%$ "Congruent" versus $20 \%$ "Real" in choice-change-to-right trials (second column) and $80 \%$ "Real" versus $20 \%$ "Congruent" in choice-change-to-left trials (third column). And in both cases no "I don't know" reports. Then if we consider choice change by pooling both trial types together, there is $50 \%$ "Real" and $50 \%$ "Congruent" reports. In other words while subjects reported a small proportion of "I don't know" as well, we expect to see at least similar proportion of real and congruent reports. Our subjects showed on average similar behavior. In choice-instructed change trials subjects reported on average $37.1 \pm 3.6 \%$ (MEAN \pm SEM) "Real" and $42.3 \pm 2.8 \%$ "Congruent" responses.

Fig. 4.2(B) gives an example that depicts why "Real" and "Congruent" reports theoretically should be $50 \%$. Imagine a scenario in which a subject selects in primary choice trials $80 \%$ the right target. We assume that the subject has the same choice preference in choice change trials. Consequently $80 \%$ of the choice-change-to-right trials should be reported as "Congruent" and $20 \%$ as "Real" because in $80 \%$ of trials (s)he already plans to select the right target. On the other hand, $80 \%$ 
of the choice-change-to-left should be reported as "Real" and $20 \%$ as "Congruent". That gives us in total $50 \%$ of choice change trials as "Congruent" and 50\% as Real. Our subjects showed on average similar behavioral outcome as expected. They reported $~ 21 \%$ "I don't know" which was mostly in change trials with short CSDs and similar total proportion of "Real" versus "Congruent" trials ( $\sim 37 \%$ vs $\sim 42 \%$ ). It is worth mentioning that for the further analysis we used only "Real" change trials otherwise it would be mentioned.

Theoretical success rate correction based on choice In FSC task we calculated the performance in change trials in two ways. One way was using only "Real" change trials based on the reports in both instructed- instructed and choice-instructed change trial types and calculate the fraction of successful changes among them.

We also thought if there is a way to calculate theoretically the real change success rates using all trails and without considering only reports. For instructed change trials we assumed that the total success rate was similar to the "Real" success rate, since the ambiguity for planning was low in those trials. But in choice change trials, we did not know the subjects' initial choice. It could be that in some trials the subjects' initial choice was congruent to the second cue. Even more complicated was the fact that sometimes subjects might not be aware of their initial plan. So there was always a subset of total choice change trials that might be not "Real" changes of plans and those trials contaminate the total success rate as we already have shown from subjects reports ig. 4.1. For choice change trials we took into account the primary choice preference to calculate the theoretically corrected success rates with this assumption that the choice preference held for one block of a task including primary and change trials. If the success rate results from theoretical method confirm the results by reports, this supports the idea that using "Real" reports for further analysis of this task would be reliable enough. The equations (1) and (2) present how we calculated the corrected success rates for choice-change-to-right and choice-change-to-left respectively. We excluded the percentage of primary choice preference congruent to the change trial type from the total success rate in that change trial type and divided the remaining by the incongruent primary choice preference. These calculations are based on the fact that the frequency subjects have to change their plans is dependent on their primary choice preference. We divided the percentage that they presumably decided to change by the total percentage that they had to change.

In choice-change-to-left trials $(=<)$, the percentage of trials that the subject had to change the plan would be equal to the percentage of right target selection in primary choice trials. To calculate the corrected success rate in this change trial type, we subtract the percentage of left target selection in primary choice trials from the total success rate in this change condition and divide it by the percentage of trials that subject had to change the plan. We calculate the corrected success rate in choice-change-to-right condition in the similar way.

To calculate the corrected overall performance in the choice-instructed change condition, including both changing to right and to left, we used the equation (3). In this equation we excluded $50 \%$ of the trials from the actual total performance, since in these trials subjects' initial plan was congruent to the instruction of the second cue (no changing trials). The other 50\% (denominator) were real changes trials. 


$$
\begin{gathered}
S R_{f r}=\text { successrate }- \text { in }- \text { choice }- \text { change }- \text { to }- \text { right }- \text { trials }(=>) \\
S R_{f L}=\text { successrate }- \text { in }- \text { choice }- \text { change }- \text { to }- \text { left }- \text { trials }(=<) \\
S R_{f C}=\text { successrate }- \text { in }- \text { choice }- \text { change }- \text { trials }(\text { both }=>\text { and }=<) \\
f C P_{R}=\text { percentage }- \text { of }- \text { right }- \text { target }- \text { selection }- \text { in }- \text { primary }- \text { choice }- \text { trials } \\
f C P_{L}=\text { percentage }- \text { of }- \text { left }- \text { target }- \text { selection }- \text { in }- \text { primary }- \text { choice }- \text { trials }
\end{gathered}
$$

$$
\begin{aligned}
& \text { CorrectedSuccessRate }_{f R}=\frac{S R_{f R}-f C P_{R}}{f C P_{L}} \\
& \text { CorrectedSuccessRate }_{f L}=\frac{S R_{f L}-f C P_{L}}{f C P_{R}}
\end{aligned}
$$

$$
\text { CorrectedSuccessRate }_{f C}=\frac{S R_{f C}-50 \%}{50 \%}
$$

Proof of (4.3):

$$
\begin{aligned}
\text { CorrectedSuccessRate } f C_{C} & =\frac{\left(S R_{f R}-f C P_{R}\right)+\left(S R_{f L}-f C P_{L}\right)}{f C P_{R}+f C P_{L}} \\
& =\frac{\left(S R_{f R}+S R_{f L}\right)-\left(f C P_{R}+f C P_{L}\right)}{f C P_{R}+f C P_{L}} \\
& =\frac{\frac{\left(S R_{f R}+S R_{f L}\right)}{2}-\frac{\left(f C P_{R}+f C P_{L}\right)}{2}}{\frac{\left(f C P_{R}+f C P_{L}\right)}{2}} \\
& =\frac{\left(S R_{\text {avg }}-0.5\right)}{0.5} \\
S R_{f C}=\frac{N_{f C \text { correct }}}{N_{f C}} & =\frac{N_{f R c o r r e c t}+N_{f L c o r r e c t}}{N_{f R}+N_{f L}}
\end{aligned}
$$

since $N_{f R} \cong N_{f L}$

$$
\begin{aligned}
S R_{f C} & =\frac{N_{f R c o r}+N_{f L c o r}}{2 * N_{f R}=2 * N_{f L}} \\
& =\frac{\frac{N_{f R c o r}}{N_{f R}}+\frac{N_{f L c o r}}{N_{f L}}}{2} \\
& =\frac{S R_{f R}+S R_{f L}}{2}=S R_{\text {avg }}
\end{aligned}
$$


Therefore:

$$
\begin{aligned}
\text { CorrectedSuccessRate } f C_{f C} & =\frac{S R_{\text {avg }}-0.5}{0.5} \\
& =\frac{S R_{f C}-0.5}{0.5}
\end{aligned}
$$

Subjects' exclusion criteria We excluded subjects if they fulfilled one of the following criteria in the FSC task Fig. 4.3: 1) If less than 10\% of instructed change trials were "Real" and correct. On average subjects (all 16 subjects) reported 85.9 $\pm 3.2 \%$ (MEAN \pm SEM) of instructed-instructed change trials as "Real" changes. That was expected because presumably after the instructed first cue subjects plan to the corresponding target which serves as the initial motor plan and it is the opposite of the second instructed cue. But on average only in $46.7 \pm 5.8 \%$ of trials subjects reported "Real" and responded to the correct target Fig. 4.3(A), black dotted line). As we required a reasonable number of correct trials for further analysis we set a minimum of $10 \%$ correct "Real" trials for exclusion criterion. 2) If less than $20 \%$ of choice change trials were "Real". On average subjects (all 16 subjects) reported $39.2 \pm 4.2 \%$ of choice change trials as "Real" changes (Fig. 4.3(B), black dotted line). The total "Real" reports in choice-instructed change trials were much lower than in instructed-instructed change trials. To have a reasonable number of trials in choice-instructed condition we set the minimum percentage of "Real" reports in this task condition to $20 \%$. In this trial type 59.5 $\pm 6.9 \%$ of "Real" reports were correct responses therefore we did not set any criterion for correct "Real" performance of choice change trials. 3) If in choice change trials, a significantly higher percentage of "Congruent" than "Real" trials was reported. One reason could be that those subjects postponed their planning so that in most of the choice change trials their initial plan was formed after and according to the second cue. Another reason could be that the subjects reported what the second cue was and not what their initial plan was. For significance testing here we used the Fisher's exact test $(\mathrm{p}<0.05)$. 

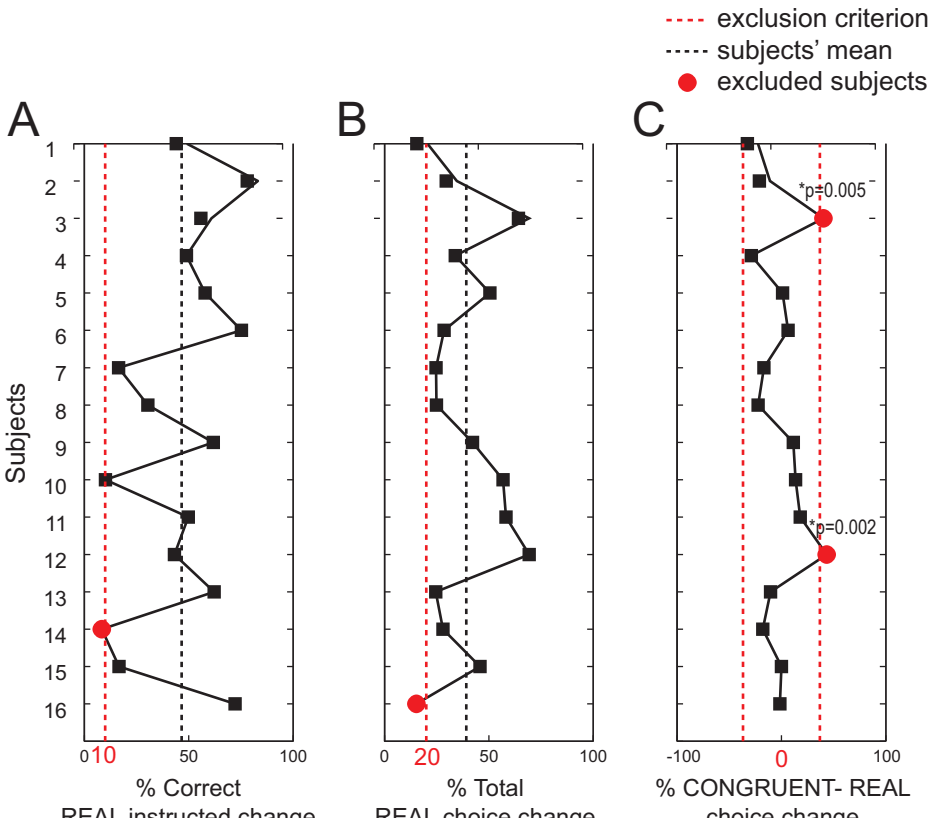

Fig. 4.3 Criteria for selecting subjects for further analysis. Sixteen subjects participated in this study. We selected subjects for further analysis based on three criteria in FSC task: Percentage of correct "Real" instructed change trials higher than $10 \%$ (A), percentage of "Real" reports in choice change trials higher than $20 \%$ (B), and significantly higher "Congruent" than "Real" reports in choice change trials $(C)$. Fisher exact test was used for the significance level $(\mathrm{p}<0.05)$ in $\mathrm{C}$. We excluded subjects who did not pass at least one of these criteria. One subject did not pass the criterion A, one subject criterion B and two subjects criterion $\mathrm{C}$. In each panel, red line shows the specific criterion and the black line the mean value across all subjects.

\subsection{Results}

We investigated the behavioral manifestations of relevant (according to task requirements) and irrelevant changes of saccade plans under instructed and free choice conditions. We hypothesized that saccade initiation is slower when there is a choice between alternative targets because multiple plans start developing after a choice cue and it takes more time for one plan to overcome the other and to continue toward execution. In addition and because of the same reason, we expected that a required reprogramming of a saccade goal in the free choice situation is faster and easier than in the instructed case. We deployed three separate tasks to address these hypotheses. Primary task included only single cued trials; FSC task included both primary and change trials in which subjects had to respond to the second cue; and FFC task included both primary and change trials but subjects had to respond to the first cue.

\subsubsection{Slower reaction time in primary choice compared to primary instructed trials}

The comparison of reaction times (RTs) between primary instructed and choice trials in all the three tasks revealed that the average choice RT was significantly slower than the average instructed RT in each of the three tasks (Fig. 4.4; in the PRM task by $14 \pm 8$ (MEAN \pm SEM) ms; in the FFC task by $39 \pm 13 \mathrm{~ms}$; in the FSC task by $18 \pm 12 \mathrm{~ms}$. A 2 -by-3 repeated measure ANOVA with the factors "task" 
and "trial type" showed significant main effects and the interaction (task: $\mathrm{F}(2,11)=35.8$, $\mathrm{p}=1.2 \mathrm{e}-7$; trial type: $\mathrm{F}(1,11)=47.2, \mathrm{p}=2.7 \mathrm{e}-5$; task $\times$ trial type: $\mathrm{F}(2,12)=11.6, \mathrm{p}=3.5 \mathrm{e}-4)$. Paired $\mathrm{t}$ test between RTs of these trial types across subjects showed that the effect was significant in all tasks (in PRM task $\mathrm{p}=0.003$; FFC task $\mathrm{p}=8.9 \mathrm{e}-6$; FSC task $\mathrm{p}=0.004$ ). This suggests that choosing between two options without any external cue takes more time compared to externally guided selections.

The overall primary RT was different across tasks. The additional requirements and the complexity of a task increased the RT. Primary task contained only primary trials and the subjects were aware of that, therefore the RTs were faster in this task compared to the two other tasks. During the FFC task, subjects should also follow the first cue, but they were distracted with a second cue in half of the trials. These irrelevant stimuli (the second cue) slowed down the RTs of the primary trials compared to the primary task (in choice trials by $53.1 \pm 11.7 \mathrm{~ms}$; in instructed trials by $28.3 \pm 8.1 \mathrm{~ms}$ ). According to the paired t-test, this effect for both trial types was significant (choice trials, $\mathrm{p}=0.004$; instructed trials, $\mathrm{p}=0.04$ ).

In the FSC task, on the other hand, subjects had to be prepared to change their movement plans in $33 \%$ of the trials. In addition, they were also monitoring their initial plans in order to be able to respond to the question in the end of change trials. The high demands of this task made the primary saccadic responses slowest among all tasks (choice RT=390.4 $\pm 13 \mathrm{~ms}$; instructed RT=371.5 \pm 10.9 $\mathrm{ms})$. Paired t test revealed that in FSC task, compared to FFC task, both trial types were significantly slower (choice trials by $48.1 \pm 13.5 \mathrm{~ms}, \mathrm{p}=4.5 \mathrm{e}-3$; instructed trials by $68 \pm 10.8 \mathrm{~ms}, \mathrm{p}=5.8 \mathrm{e}-5$ ).

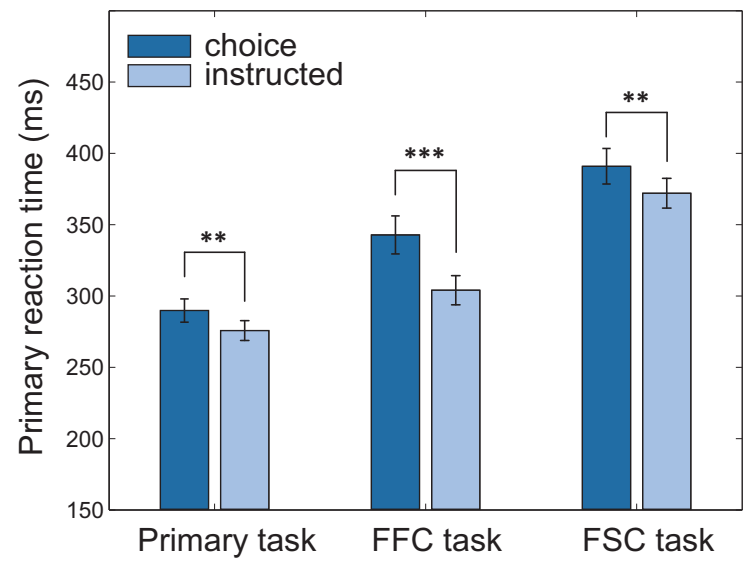

Fig. 4.4 Slower reaction time in primary choice than in primary instructed in all tasks. Comparison between primary instructed (light blue) versus primary choice (dark blue) reaction times in each task. Reaction times were calculated from the appearance of first cue. Error bars indicate standard error of means (SEM). Significance level were tested by paired $t$ test $(* \mathrm{p}<0.05, * * \mathrm{p}<0.01, * * * \mathrm{p}<0.001)$.

\subsubsection{Follow second cue task (FSC task)}

FSC task was designed to study the behavioral differences of saccade planning and execution when new information requires a saccade goal update in instructed and free choice situations. In most of the trials of this task subjects had to saccade toward one of the two bilaterally presented targets based on a single instructed or free choice cue (primary trials), as in the primary task. But in a subset of trials, a second instructed cue replaced the first cue after a certain delay (change trials), and subjects were 
required to update the saccade plan to the new relevant target. Since in choice change trials we were not aware of the subject's initial plan and therefore we did not know whether the subject really had to change the plan or not, we asked subjects to report their initial saccade plan (before the second cue) in each change trial by a keyboard button press. This helped us to isolate, according to subjects' reports, the trials in which "Real" changes of plans happened, from the "Congruent" trials (no actual change) and also from those in which subjects were not aware of or did not remember the initial plan ('I don't know' report). We then compared performance as well as the reaction times in choice change versus the instructed change trials. Our data show that subjects were faster and better in performing choice change trials in comparison to instructed change trials. This suggests that when two alternative plans are present reprogramming an action is easier and faster than switching between two opposite saccade goals.

\section{Better change performance in choice trials as compared to instructed trials}

Having isolated the trials where real changes of plan took place, we next asked how well subjects performed the task in different change trials. We compared the success rate in "Real" instructed change versus "Real" choice trials. Across all CSDs, subjects had slightly better performance in the choice change trials as compared to the instructed change trials, but the difference did not reach significance (Fig. 4.5(A); 59 $\pm 7 \%$ (MEAN \pm SEM) versus $52 \pm 6 \%$; paired $t$ test $\mathrm{p}=0.14$ ). The comparison for each CSD separately showed that in all CSDs except for the longest one, subjects performed better in changing a plan after a choice rather than an instructed cue Fig. 4.5(C). We applied two factor (trial type and CSD) repeated measure ANOVA. There was a main effect of trial type $(\mathrm{F}(1,11)=9.01$, $\mathrm{p}=0.01)$ and $\operatorname{CSD}(\mathrm{F}(5,11)=45.4, \mathrm{p}=2 \mathrm{e}-17)$ but no interaction between them $(\mathrm{F}(5,11)=1.2, \mathrm{p}=0.34)$. We also used paired $t$ test between success rates of two trial types in each CSD. There was a significant difference between success rates only for the two middle CSDs $(\mathrm{CSD}=134 \mathrm{~ms}, \mathrm{p}=0.02 ; \mathrm{CSD}=184$ $\mathrm{ms}, \mathrm{p}=0.009$ ). This result supports the hypothesis that during a choice between two potential targets, both option plans are available and if the plan for one target is initiated, it is easier to switch to another target as compared to situations in which there is only one plan (for an instructed target) and a change to an opposite target is required. In addition success rate decreased with increasing the CSD Fig. 4.5(C). In long CSDs (251 ms and $301 \mathrm{~ms}$ ) the success rate was on average less than $40 \%$. This demonstrates that when the change signal delay is long, the initial saccade plan might be already in its later stages of motor preparation and close to be executed, and hence changing it is difficult.

Similarly to the analysis above, Fig. 4.5(B,D) depict the performance in instructed change trials compared to choice change corrected based on the primary choice preference. On average, subjects had significantly better performance in choice change trials (Fig. 4.5(B); 68 $5 \%$ versus $56 \pm 5 \%$; paired $t$ test $\mathrm{p}<0.001)$. We also compared performance in each CSD separately Fig. 4.5(D). The two factor (trial type and CSD) repeated measure ANOVA showed that there were significant main effect for both factors and the interaction (Trial type: $\mathrm{F}(1,11)=23.6$, $\mathrm{p}=5.1 \mathrm{e}-4$; $\mathrm{CSD}: \mathrm{F}(5,11)=78$, $\mathrm{p}=1 \mathrm{e}-23$; trial type $\times$ CSD: $F(5,11)=4.3, p=0.002)$. According to the paired t test between two trial types in each CSD, in the first four CSDs the choice change trials showed higher success rates than instructed change trials $(\mathrm{CSD}=17 \mathrm{~ms}, \mathrm{p}=0.003 ; \mathrm{CSD}=67 \mathrm{~ms}, \mathrm{p}=0.003 ; \mathrm{CSD}=134 \mathrm{~ms}, \mathrm{p}=0.0001 ; \mathrm{CSD}=184 \mathrm{~ms}, \mathrm{p}=0.02)$.

Thus, our data showed the same pattern of success rates with both calculation methods, using only "Real" change self-reports and using the correction method of a total success rate by primary choice 
preferences. In both cases, success rate in the choice change trials was higher than in the instructed change trials for most of the CSDs. This confirms that the reported "Real" changes were very close to what we expected to see based on the theoretical correction method. Therefore for further change reaction time (CRT) analysis we relied on the reports and used only "Real" choice change and "Real" instructed change trials.

\section{Faster choice as compared to instructed CRT}

We were also interested to inspect whether the reaction times differed in choice change versus instructed change trials. For CRT analysis, we considered only "Real" change trials isolated by subjects' reports. The CRT was significantly faster than the RT in choice trials (Fig. 4.6(A) ; $41 \mathrm{~ms}$; paired ttest $\mathrm{p}=0.006$ ), but not in instructed trials $(-5 \mathrm{~ms}$; paired $\mathrm{t}$ test $\mathrm{p}=0.63)$. This implies that only when two alternative plans are present, reprogramming an action after a spatial goal update, during the motor planning stage, is faster than planning and initiating a single action.

A

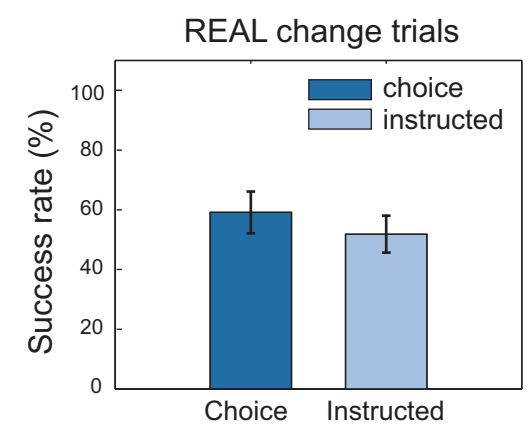

C

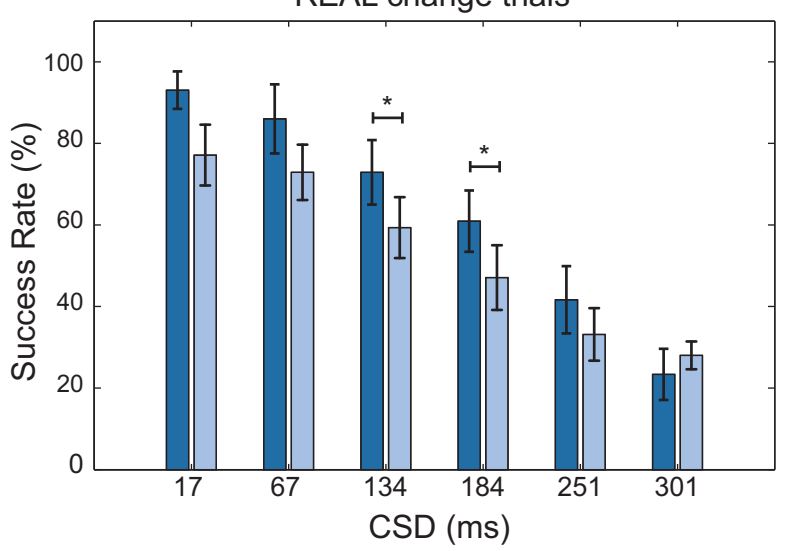

B

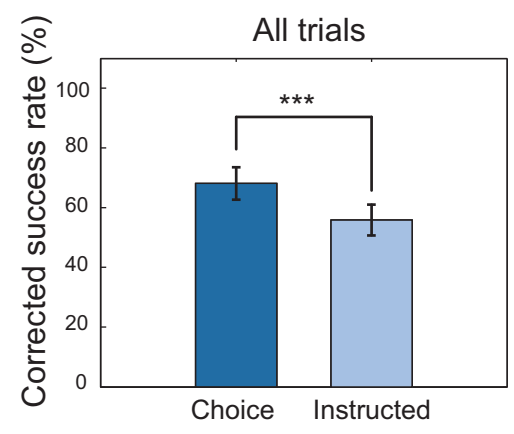

$\mathrm{D}$

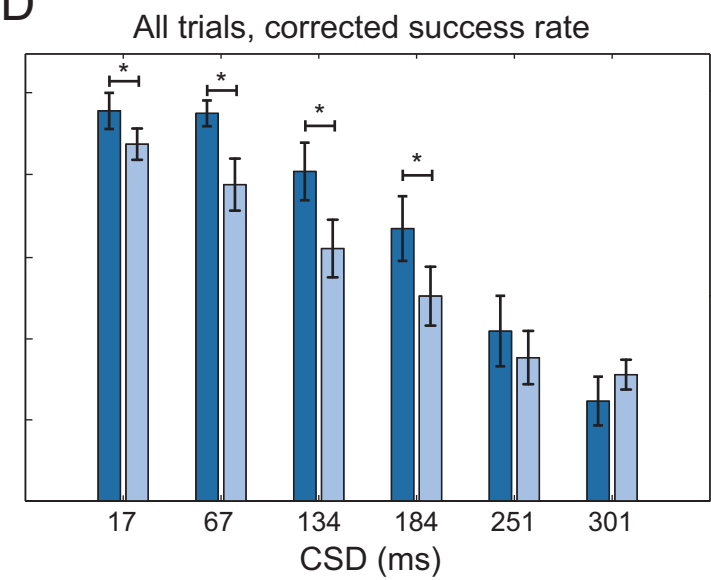

Fig. 4.5 Performance in choice change compared to instructed change trials in the FSC task. Success rates were calculated by two approaches: using "Real" change self-reports (A, C) and by theoretical correction based on target selection preference in primary choice trials $(B, D)$. A and B represent total success rate across all CSDs while $C$ and D show success rate in each CSD separately. Dark blue represent performance in choice change trials and light blue the performance in instructed change trials. Error bars indicate SEM. Significance level were tested by paired $\mathrm{t}$ test $\left({ }^{*} \mathrm{p}<0.05\right.$, ** $\left.\mathrm{p}<0.01, * * * \mathrm{p}<0.001\right)$.

The other interesting point here is that choice CRT was significantly faster compared to instructed primary RT ( $23 \mathrm{~ms}$; paired $\mathrm{t}$ test $\mathrm{p}=0.046$ ), but in instructed trials the averaged CRT was slightly 
slower than RT Fig. 4.6(A). This suggests that in choice change trials, although the initial intention was to saccade to the target incongruent to the second instructed cue, the motor preparation was done partially for both targets, resulting in the observed faster CRT.

In addition, choice CRT was significantly faster than instructed CRT by $28 \mathrm{~ms}$ (Fig. 4.6(A); paired $t$ test $p=0.035$ ). Choice cue triggers planning for both potential targets and therefore this result implies that presence of two potential plans allows faster update of a plan than in instructed change trials.

Fig. 4.6(B) illustrates the comparison between instructed and choice CRT for each CSD separately. According to the two factor (trial type and CSD) repeated measures ANOVA, there were no significant main effects and also the interaction (Trial type: $F(1,11)=1.6 e-31 \mathrm{p}=1$; $C S D: F(5,11)=8.7 \mathrm{e}-31 \mathrm{p}=1$; interaction: $\mathrm{F}(5,11)=6.3 \mathrm{e}-31 \mathrm{p}=1)$. But when we calculated the same ANOVA for only seven subjects where we had data for all CSDs in both trial types, the result was different. This analysis showed only significant effect of interaction (Trial type: $F(1,6)=4.75$, $p=0.07$; CSD: $F(5,6)=2.5, p=0.052$; interaction: $F(5,6)=2.78, p=0.035)$. For the CSDs 67, 134, 184 and $251 \mathrm{~ms}$, choice CRT was faster than the corresponding instructed CRT (only significant for $\mathrm{CSD}=251 \mathrm{~ms}$; paired ttest $\mathrm{p}=0.005$ ).

A

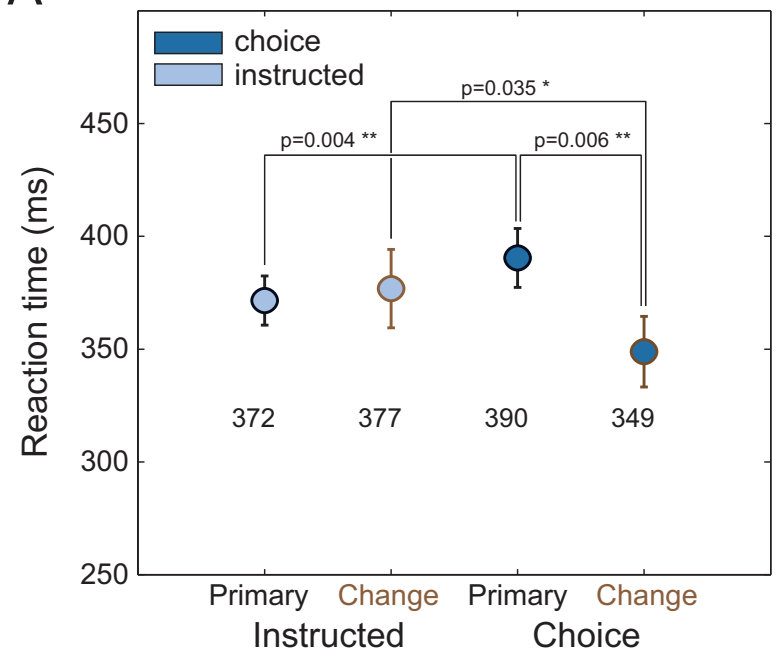

B

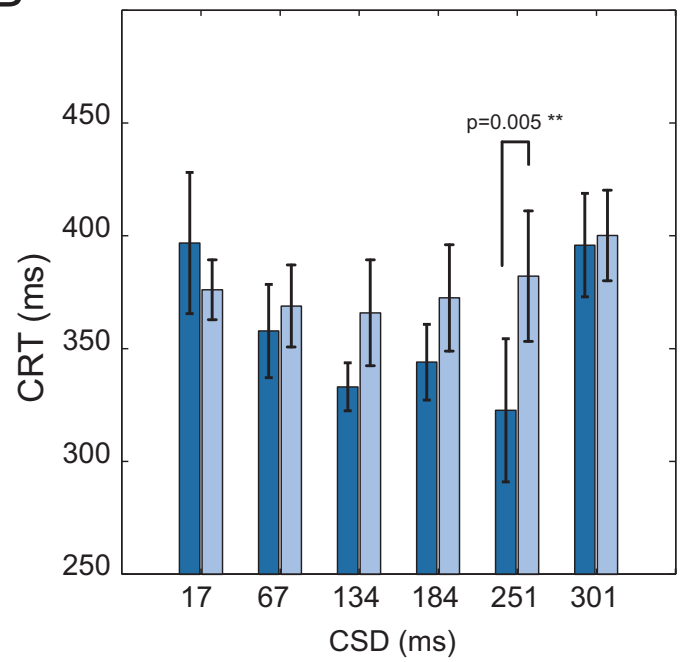

Fig. 4.6 Reaction times in the FSC task. A) Average primary versus change reaction times in choice and instructed trials. B) Instructed (light blue) versus choice (dark blue) change reaction time (CRT) in each CSD separately. CRTs are calculated from the onset of the 2 nd cue. Error bars indicate SEM. Significance was tested by paired t test $\left(* \mathrm{p}<0.05\right.$, $* * \mathrm{p}<0.01$, ${ }^{* * *}$ $\mathrm{p}<0.001)$.

\subsubsection{Follow first cue task (FFC task)}

In many real life situations, changes in the environment usually require to plan a new movement but in some occasions changes are irrelevant and new actions should be avoided. We used FFC task to investigate the behavior during irrelevant visual changes in conditions in which subjects should inhibit updating plans. In this task subjects had to respond based on the initial information (first cue) and in the case of change trials ignore the irrelevant second cue (distracter).

FFC task also served as a control to check whether subjects could perceive the first cue in choice change trials with shortest CSDs in the FSC task. In this task, in the choice change trials, it could be that the first cue (=) was backward masked by the second cue (an arrow). If this were the case, 


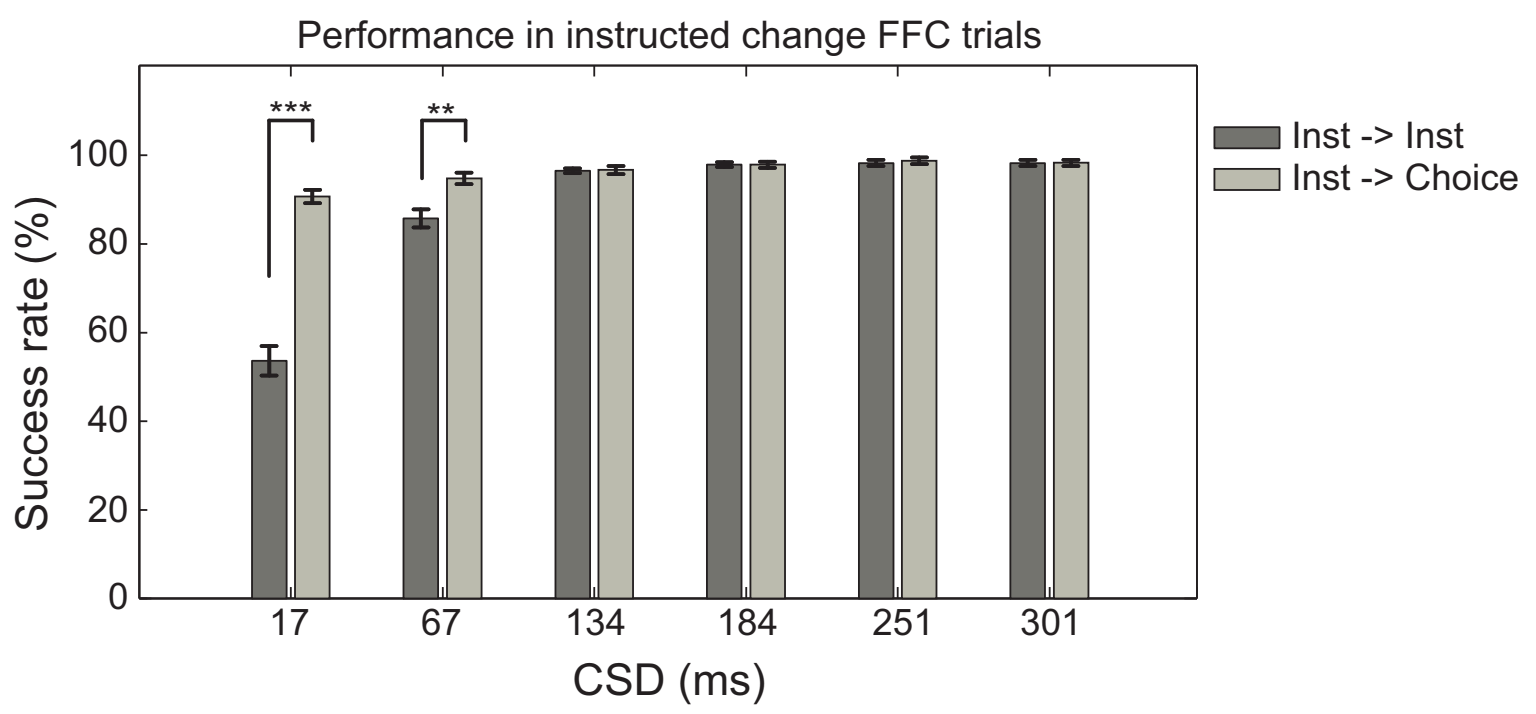

Fig. 4.7 Performance in instructed-instructed and instructed-choice trials in FFC task. Proportion of correct responses based on the requirements of the first cue in FFC task for two conditions: when an instructed cue changes to another instructed cue (e.g. instructed left change to instructed right; dark color); when an instructed cue changes to a choice cue (light color). Since the second cue is irrelevant, we expected to see no difference in performance between two trial types. But the performance dropped in the first two fast CSDs differently for each condition. Error bars indicate SEM. Significance level was tested by paired $\mathrm{t}$ test $(* \mathrm{p}<0.05, * * \mathrm{p}<0.01, * * * \mathrm{p}<0.001)$.

subjects could not perceive the first cue and therefore the performance for short CSDs in choice change trial would be high.

\section{Only “instructed" second cue affected performance at short delays}

Fig. 4.7 depicts the performance in two different types of change trials in the FFC task: instructedinstructed (arrow - arrow) and instructed-choice (arrow - =) change conditions. The performance in the shortest CSD dropped dramatically when the second cue was an instructed cue, an arrow $(53 \pm 3.5 \%$ MEAN \pm SEM) but not when it was a choice cue $(90 \pm 1.5 \%)$, and also dropped slightly for the second CSD (second cue instructed: $86 \pm 2.1 \%$, second cue choice: $95 \pm 1.3 \%$ ). According to the two factor repeated measure ANOVA, there was a significant main effect of the two factors (trial type and CSD) and the interaction on the success rate (trial type: $F(5,11)=121.3, p<1 e-7$; CSD: $\mathrm{F}(1,11)=134.4, \mathrm{p}=1.7 \mathrm{e}-7$; trial type*CSD: $\mathrm{F}(5,11)=52$, $\mathrm{p}<1 \mathrm{e}-7)$. In addition, paired t test revealed that the performance in two trial types differed significantly only in the two shortest CSDs (CSD $=17 \mathrm{~ms}$, $\mathrm{p}=1.31 \mathrm{e}-6 ; \mathrm{CSD}=67 \mathrm{~ms}, \mathrm{p}=0.0014)$. These results suggest that the first arrow cue was not masked by the second choice cue (=), and the performance drop in the instructed-instructed trials was due to automatic orienting to a behaviorally salient second arrow.

\section{Irrelevant "instructed" second cue affected choice preference and RT}

Our main goal in FFC task was to study the behavioral outcomes of irrelevant changes. We looked at the choice preference and RTs of target selection in the choice-instructed change trials for each CSD to investigate the effect of the instructed second cue on choice behavior. According to the two 
factor (trial type and CSD) repeated measure ANOVA, there was a main effect only for the trial type factor and the interaction $(\mathrm{CSD}: \mathrm{F}(5,11)=1.9 \mathrm{e}-12, \mathrm{p}=1$; Trial type: $\mathrm{F}(1,11)=78.0, \mathrm{p}=2.5 \mathrm{e}-6$; interaction: $F(5,12)=21.1, p=1.0 \mathrm{e}-11)$. We used paired t test to compare the two trial types in each CSD. The averaged choice preferences were different only in the first three CSDs (CSD $=17 \mathrm{~ms}$, $\mathrm{p}=2.4 \mathrm{e}-6 ; \mathrm{CSD}=67 \mathrm{~ms}, \mathrm{p}=1.5 \mathrm{e}-4 ; \mathrm{CSD}=134 \mathrm{~ms}, \mathrm{p}=1.9 \mathrm{e}-5$ ). Thus, subjects showed significantly more selection of the targets congruent to the instructed second cue as compared to the opposite targets, in the first three CSDs Fig. 4.8(A). 
A
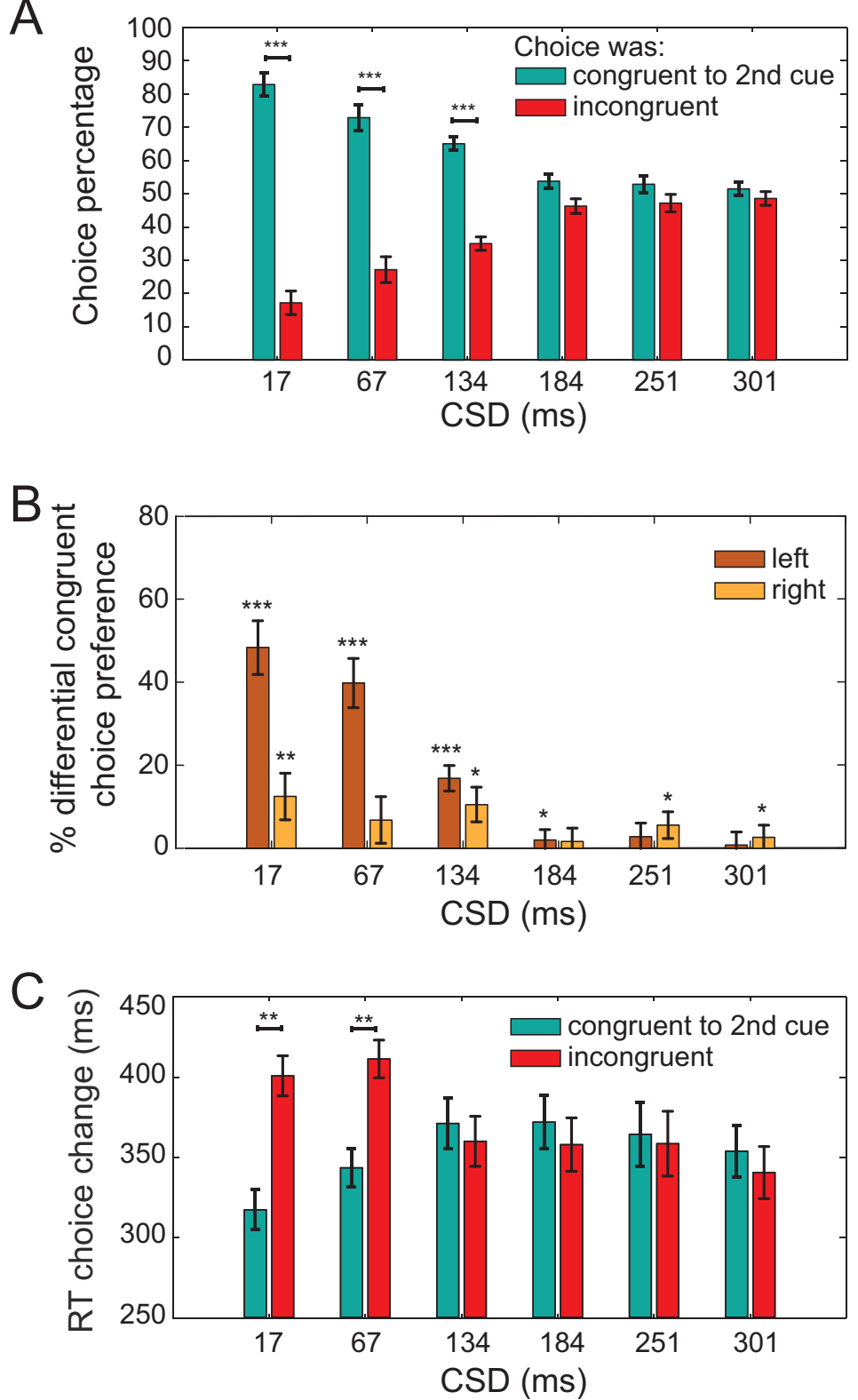

Fig. 4.8 Influence of the second irrelevant instructed cue on the choice proportion and RT in FFC task. A) Proportion of target selection in choice-instructed trials for targets congruent to the instructed second cue (turquoise) versus opposite targets (red). B) Difference in proportion of left (orange) and right (yellow) target selection in choice-instructed trials relative to the primary choice trials. C) Reaction time of saccades toward targets congruent to the second cue (turquoise) versus toward the opposite target (red) in choice-instructed trials. Error bars indicate SEM. Significance level was tested by the paired t test $\left(* \mathrm{p}<0.05,{ }^{*} \mathrm{p}<0.01,{ }^{* * *} \mathrm{p}<0.001\right)$. In (B) the test was between choice proportions in choice-instructed vs. primary choice conditions.

We next compared the target selection in primary choice condition versus the choice-instructed change condition. Since subjects had to perform the task based on the first cue (thus operationally choice-instructed FFC trials are the same as primary choice trials), by this comparison we intended to see if the irrelevant second cue affected the choice proportion directly. Fig. 4.8(B) shows the proportion of the congruent left and right target selection in choice-instructed trials relative to primary 
choice trials. Values close to zero indicate that the choice proportions did not differ in these two conditions. We tested this effect using two separate two factor (trial type and CSD) repeated measure ANOVAs, one comparing left choice proportion in choice-change-to-left trials versus in primary choice trials (trial type: $F(1,11)=63.6 p=6.74 \mathrm{e}-6$; $C S D: F(5,11)=25.6 \mathrm{p}=3 \mathrm{e}-13$; interaction: $\mathrm{F}(5,11)=25.6$ $\mathrm{p}=3 \mathrm{e}-13$ ), and the second comparing right choice proportions (trial type: $\mathrm{F}(1,11)=5.6 \mathrm{p}=0.038$; CSD: $F(5,11)=2.96 p=0.02$; interaction: $F(5,11)=2.96 p=0.02$ ). For both left and right choices, there were significant main effects of both factors and the interactions, but the effects were stronger for left choices. Only in the three shortest CSDs the irrelevant second cue modified the choice target selection, and the effect was higher for left choices than for right choices. The reason for the left/right asymmetry was that all subjects except one had a preference for the right target selection in the primary choice trials (estimated across both sessions for each subject), therefore there was not a large difference between proportions of right choices in the primary trials and those "imposed" by the second instructed cue.

Aside from the choice proportions, subjects also initiated saccade significantly faster to targets congruent to the second cue, in the first two shortest CSDs (Fig. 4.8(C); CSD=17 ms, p=9e-3; CSD=67 $\mathrm{ms}, \mathrm{p}=9 \mathrm{e}-3)$. According to the two factor repeated measure ANOVA, the main effect existed only for the interaction of the trial type and the CSD (CSD: $\mathrm{F}(5,11)=2.02, \mathrm{p}=0.09$; trial type: $\mathrm{F}(1,11)=4.3$, $\mathrm{p}=0.06$; interaction: $\mathrm{F}(5,11)=10.3, \mathrm{p}=5.4 \mathrm{e}-7)$.

\subsection{Discussion}

The ability to flexibly adapt behavior to a new visual input is a hallmark of primate behavior. Humans are able to change or stop an action, depending on if the new input is currently relevant or not, in a very short time. Here in this study we target this kind of behavior by using a change-signal paradigm (Jha et al., 2015; Nachev et al., 2005) which is a modified version of stop-signal paradigm (D. Logan $\&$ B. Cowan, 1984). More specifically we studied three interesting aspects of human saccade action selection: free-choice target selection, changing a saccade plan or inhibiting an irrelevant new stimulus during instructed or free-choice action selection. Up to our knowledge this is the first behavioral study that investigates detailed behavioral aspects of saccadic goal updates while a saccade is already planned volitionally compared to a externally-driven saccade.

First, we compared a single saccade target selection in free-choice versus instructed context. We showed that when subjects had to select a target between two bilaterally available options, they were slower if they were free to choose than externally instructed to select one. This was consistent across all three tasks (Primary, FSC and FFC). This suggests that choosing between two options without any external force takes more processing time to resolve the competition between options compared to externally-guided selections. The overall primary RT was different across tasks. The requirements and the complexity of a task increased the RT.

Updating a motor plan has been studied extensively since decades ago mainly in the context of countermanding or response inhibition tasks. Several paradigms has been used to understand the underlying processes of stopping a current plan using different methods such as fMRI, MEG, EEG and electrophysiology (Greenhouse \& Wessel, 2013; Jha et al., 2015; Nachev et al., 2005; Pouget et al., 2011; Ramakrishnan et al., 2012) as well as computational modelling (Band et al., 2003; Boucher 
et al., 2007; Camalier et al., 2007; Hanes \& Carpenter, 1999). Also in parallel many studies focused on how motor plans and decisions might change to a new relevant goal mainly to resolve whether changing a plan require a separate stop process to cancel the current plan or not (Boecker et al., 2013; Jha et al., 2015; Nachev et al., 2005; Verbruggen \& Logan, 2008a; Verbruggen et al., 2008). Although it has been shown that stop process might partially overlap with the brain areas processing action reprogramming (Boecker et al., 2011), but several behavioral studies suggest presence of a separate stop process for response inhibition and switching (Camalier et al., 2007; Verbruggen \& Logan, 2008b; Verbruggen et al., 2008). In the current study as mentioned, we used more cognitive change-step paradigm with symbolic cues in two contexts, in one subjects had to follow the signal of the second cue (FSC task) and in another subjects had to follow the first cue and ignore the second irrelevant cue. In none of these tasks subjects confronted with an explicit stop cue and inhibiting an incorrect action was implicitly processed by them.

In our main task (FSC) subjects had to change their ongoing saccade planning, emerged either by having free-choice or being instructed, to another option in a subsequent of trials. Since we monitored subjects' initial succade plans, we could calculate success rates with two methods, in one method we trust subjects' report and in another we assume that the primary choice preference stays the same for the choice change trials. The results from both methods were similar. We observed that subjects had better performance in the choice change trials as compared to the instructed change trials. Across different delays,only when the delay was too long (301 ms) subjects did not perform better in choice-change trials. This result supports the hypothesis that during a choice between two potential targets, both option plans are available (Cisek \& Kalaska, 2005; Klaes et al., 2011) and if the plan for one target is initiated, it is easier to switch to another target compared to situations in which there is only one plan (for an instructed target) and a change to an opposite target is required.

In this task, subjects' change reaction time (CRT) was significantly faster than the primary RT in choice trials but not in instructed trials $(-5 \mathrm{~ms})$. This implies that only when two alternative plans are present, reprogramming an action after a spatial goal update, during the motor planning stage, is faster than plan and initiate a single action. In addition, CRT in choice was faster than in instructed trials (consistent in all CSDs except the shortest $(17 \mathrm{~ms})$ and longest $(301 \mathrm{~ms})$ ). Choice cue triggers planning for both potential targets and therefore this result implies that presence of two potential plans allows faster update of a plan than in instructed change trial.

In a fMRI study, (Nachev et al., 2005) used change-signal paradigm to understand the brain regions involved in free-choice and also change of instructed or choice plans. Although their paradigm seems very similar to our FSC task but there are several differences to our design. They separated the plan cue (first) and the change cue (second) by a separate "go" cue but in our design the cue changed abruptly to another cue. Also unlike our design their second cue was a "change" or "stay" signal that is cognitively different from our design in which we presented a cue toward other direction. Their design does not allow a reliable latency measurement to compare between conditions. However, their results show a higher saccade latency in free-choice than instructed trials and slower latency in changing from choice than instructed. And in general they argue that their behavioral results is consistent with the race model predictions (Hanes \& Carpenter, 1999). By the way (Jha et al., 2015) designed a similar but more complex MEG study to investigate the functional role of related brain areas in stopping or changing instructed finger movements. In their task a primary instructed cue was presented and after 
that another cue used to inhibit the current action or change response to opposite option. They found activation of frontal areas pre-SMA and IFG in this task which is consistent to several previous reports which associated those areas for learning actions and updating or suppressing a motor plan (Gremel \& Costa, 2013; Nachev et al., 2005; Neubert et al., 2010; Obeso et al., 2013; Rushworth et al., 2002). As mentioned most of the studies before investigated the behavior of changing actions in contexts similar to our instructed-change conditions. Mostly tasks such as step-signal (Becker \& Jürgens, 1979) or its modified version redirect tasks (Camalier et al., 2007; Ray et al., 2004) were used in which saccadic target selection to a new salient visual cue is required. In addition, the behavioral aspects of changing plans in a free-choice condition was missing.

Another type of experiments that are similar to our paradigm is visually-guided step-signal paradigms. In those studies, usually a visual cue appears in one part of visual field and after a variable short time it jumps to another location. Subjects have to sometimes follow both cues or just the second cue. (Ray et al., 2004) studied reprogramming of saccades to an updated target location using two tasks. They presented two consecutive targets with a delay in between to human subjects and asked them either to make a saccade directly to the second cue (redirect task) or saccade to both targets (follow task). They found that a corrective saccade is faster when the delay between targets is shorter and it is faster in redirect task than follow task. Their data supports the idea that brain has the capacity to program two saccades in parallel than in a serial way. In a similar study using double step task, (Camalier et al., 2007) investigated whether the behavior of human and monkey subjects differ when the updated saccade executed under the presence of other distractors in the visual field (called as search step task). They found also that the error rate increases for longer delays between targets. They also found that corrective saccades tend to be faster than the average primary (no-step) saccades. This is partially consistent with our data. We showed that the change-RT is not different from primary-RT in FSC task for the instructed-change trials but it is significantly faster for choice-change trials especially when the delay between targets is not too short $(17 \mathrm{~ms})$ or too long (310ms). The shortest and longest delay in (Camalier et al., 2007) are $89 \mathrm{~ms}$ and 230ms and in (Ray et al., 2004) are 50ms and 200ms so that their range of delays correspond to out middle delay times. One of the reasons for this difference could be due to the fact that we used symbolic cues rather than salient spatial visual cues and that costs more cognitive efforts. (Camalier et al., 2007) and (Ramakrishnan et al., 2012) modeled the behavior in these tasks and found that the performance could be better explained by applying a separate stop process in addition to two go processes corresponding to two saccade plans. This stop process interrupts the first saccade plan and let the second saccade further processing. It has been argued that the duration of stop process which is needed to suppress a saccade preparation or execution is between $100-150 \mathrm{~ms}$ according to the neurophysiological data (Ramakrishnan et al., 2010).

To dissociate the perceptual and motor-planning of choice behavior (Shankar et al., 2011; Stanford et al., 2010) designed a compelling-saccade task. They first signaled the spatial location of two potential choice targets and then with a separate "go" signal asked monkeys to start movement planning. After a short variable gap, target's color changed and instructed the correct target to monkey. Similar to our data for choice-change trials in the FSC task they showed decreased of success rate to chance level when the gap between gap and target increases. But opposite to our results, they show slight increase in RT as the gap gets longer. This is consistent to our instructed-change trials. In our 
choice-change trials, we do not separate the perception, decision and motor response but in their task they separate the decision process by the motor preparation by the "go" signal. It is also likely that monkeys plan for both targets instead of selecting just one target before go signal. Therefore, we cannot entirely compare their results with our choice-change results.

One reason that we added FFC task to our experiment was because during pilot experiments with the FSC task we were not sure if subjects could perceive the first cue in choice change trials with shortest CSDs (17 and $67 \mathrm{~ms}$ ). In choice change trials the second instructed cue overlapped with the half of the choice cue but in instructed change trials two instructed cue were presented beside each other without overlap. Therefore, the high success rate in choice change trials for shortest CSDs could be explained by the fact that the first cue was completely masked by the second cue. The results from the FFC task reject this hypothesis.

The performance in the shortest CSD (less than $100 \mathrm{~ms}$ ) dropped dramatically when the second cue was an instructed but not when it was a choice cue. We offer two potential, not mutually exclusive, explanations for this result. First, there seems to be a degree of perceptual masking for instructed-choice trials when the change delay is very short. On the other side we saw the decrease in performance in instructed-instructed more than in instructed-choice. Our explanation for this effect is that humans tend to follow the direction of arrows as a rule and therefore subjects might saccade reflexively when the second cue instructs them after a short delay, and the initial plan is not yet fully formed.

On the other side, in the FSC task the main difference between "real" choice and instructed change CRT and also success rate was in the middle length CSDs but not for very short CSDs. So it confirms that even if perceptual masking effect happens it does not affect our main results. In addition, FFC task by itself is interesting to understand the time constraint of influence of an irrelevant sensory cue on the current saccade plan. A new sensory information is sometimes just a distractor and is not behaviorally relevant therefore it needs to be ignored and inhibited.

We also observed that a second irrelevant instructed cue can influence choice preference of subjects when it is presented in less than $150 \mathrm{~ms}$ after a choice cue. In spite of this the reaction times were affected only for the delay less than $100 \mathrm{~ms}$. In short delays only a proportion of choices congruent to the second cue are influenced by the second cue and the rest are subjects' internal choice for that option. Those influenced choices are the ones with longer reaction times because for those a reprogramming was needed. But in longer delays $(\mathrm{CSD}=134 \mathrm{~ms})$ preparation for both options developed largely so that reprogramming was easier for those influenced choices. Therefore, the effect on RT disappeared. Together those results suggest that an irrelevant change might have an effect on the choice behavior only if it happens within $150 \mathrm{~ms}$ after the first cue. It seems that the choice target selection was vulnerable to be affected for a bit longer time than saccade latencies during motor preparation.

\subsection{Acknowledgments}

We especially thank Malte Koester for programming the task and analysis preprocessing in the early stages of the project. We also appreciate Dr. Caio Moreira to have conceptual helps and discussions. We admire and thank a lot Kristin Kaduk to help us conceptually and collect most of the data from 
our human subjects in different stages of the project. We appreciate Ira Panolias, Klaus Heisig and Ralf Brockhausen for their technical supports. 



\section{Chapter 5}

\section{General discussion}

Ever-changing environment requires flexible action selection for survival and the primate brain has been evolved to overcome this efficiently. It has been proposed that for a visually-guided action selection multiple possible choices are represented by discrete neuronal populations within frontoparietal network and modulatory signals from other cortical or subcortical brain regions can influence the decision process such that eventually one action will be executed ("Affordance competition hypothesis" (Cisek, 2012)). One of the potential modulatory inputs is from thalamus with its vast anatomical connections with almost all brain cortical areas which suggests that thalamus is important for mediating cortical activities in several stages of information processing. For example pulvinar which is the largest nucleus in thalamus has been shown to causally influence cortical activities and its perturbations can cause severe visuomotor deficits and has been shown to modulate attention-related cortical activities.

This thesis includes studies which help us to understand reach and saccadic action selection process behaviorally and neurophysiologically. We investigated the role of neuronal responses in dorsal pulvinar ( $\mathrm{dPul}$ ) compared to the parietal cortex for visually-guided reach and saccade behavior. In addition, we investigated the causal role of $\mathrm{dPul}$ in eye-, hand- and spatial neuronal representations by applying electrophysiological and pharmacological methods. Moreover, in a human psychophysics research we studied the visually-guided saccadic free-choice action selection and the influence of relevant or irrelevant new visual stimulus in changing saccade plans.

In our first electrophysiological study, we recorded neuronal activities from cortical area MIP and in separate sessions from subcortical structure dPul while two macaque monkeys performed dissociated delayed saccade and reach (extra-foveal) and dissociated free-gaze reach (foveal) tasks using either left or right arm. Those tasks were performed in separate blocks within each session. We asked how effector-specific (eye vs left- vs right hand) as well as task-specific (saccade vs reaches) are local field potentials in those regions.

Although several previous studies showed limb- and space-specificity of MIP and PRR spiking activities in reach and saccade tasks (for example (Chang et al., 2008; Chang \& Snyder, 2012; Mooshagian et al., 2017)), no study systematically characterized hand responses in LFP oscillations (however see (Dean et al., 2012; Hwang \& Andersen, 2011, 2012; Scherberger et al., 2005; Stetson $\&$ Andersen, 2014)). In addition, current knowledge about functional contribution of the dPul in goal-directed reach behavior is scarce. There is no study about LFP encoding of eye-, hand- and space 
in dPul (see a few studies only on spikes (Acuna et al., 1990, 1983; Cudeiro et al., 1989)) and this is the first study which addressed the same questions in dPul LFP oscillations.

We found that dPul and MIP represent both hands and share overall similarities in their power spectra (especially within each monkey), with a peak modulation in the beta band $(12-30 \mathrm{~Hz})$ and weaker modulation in theta $(4-8 \mathrm{~Hz})$ and gamma $(30-120 \mathrm{~Hz})$ bands. However, subcortical structure dPul seems to have a lower raw power than in MIP which was expected (Buzsáki et al., 2012). Interestingly both MIP and dPul has contralateral spatial tuning for the cue presentation in the lower frequency bands (theta-beta) which is consistent across tasks. Although gamma band only in one monkey shows contralateral delay period activity (stronger in MIP than in dPul), but gamma band power before and during movement is slightly higher for movements to contralateral space in both MIP and dPul and interestingly stronger in reach than saccade. We need to investigate more whether this might be related to general higher firing rates in reach than saccade. Interestingly the data from (Hwang \& Andersen, 2011, 2012) shows higher gamma band activity in a similar task most probably because of the recordings in anatomically different locations. Our data from monkey $\mathrm{T}$ with recordings from more posterior locations in MIP confirms this hypothesis because there we see more gamma band activity than anterior locations.

The most striking activity is the beta band in both regions. However, consistent with previous reports in motor cortical areas, beta band power in MIP around the movement time shows a rebound with strong decrease of power during an active or imaginary movement and increase after the movement finishes which has been associated with GABAergic inhibition (Fry et al., 2016; Jensen et al., 2005; Jurkiewicz et al., 2006; Pfurtscheller et al., 2005). Interestingly dPul did not show this pattern which implies that dPul might have less motor-like but complex response .

It has been shown before that beta band increases during steady states and movement planning periods but decreases during movement (Dean et al., 2012; Hagan et al., 2012; Hwang \& Andersen, 2011). Our data shows that in both regions this beta band modulation is stronger when monkey performs reaches with contralateral hand. This is a sign for reach-specificity of pulvinar similar to MIP.

In addition, we showed that when the monkeys were allowed to look during reach (free-gaze task), power spectrum in both regions just slightly changed compared to complete dissociated reaches. This might be because the level of beta band reflects the computational load for the eye-hand sensorimotor transformations and therefore less transformational load causes higher beta levels. It is interesting that $\mathrm{dPul}$ also shows this effect similar to MIP.

Moreover, our tuning analysis for the dissociated reach task revealed that in both regions, beta band mostly reflects the hand tuning in contrast to gamma and theta bands in which they mostly reflect the spatial tuning and to less degree hand tuning. Although the percentage of spatially tuned LFP sites in theta was higher in dPul than MIP during stimulus presentation, slightly more LFP sites in MIP than dPul were tuned in gamma band during cue, delay and movement periods.

We also studied the reach and saccade representations in posterior compared to anterior locations in MIP. Those locations seems to have different anatomical connections to separate parts within PPC and visual or frontal cortices, do different levels of sensorimotor transformations and motor-goal planning and also lesions in those areas caused different visuomotor deficits (Battaglia-Mayer et al., 2016; Caminiti et al., 2010). Therefore, it would be expected that the level of limb- and task-specificity along 
the intraparietal sulcus changes. Our data from one monkey shows that beta band power decreases in the delay period during reach vs saccade planning much stronger in anterior locations than posterior locations (stronger for ipsilateral arm). In addition, contralateral hand selectivity in this band increases more in anterior locations. On the other hand, during late delay and movement, anterior LFP sites does not show task specificity as much as the posterior LFP sites in the gamma band. There is a strong increase in gamma power for contralateral reaches compared to contralateral saccades in posterior sites (contralateral space selectivity). But in the anterior MIP, this effect was much weaker. These results confirm that posterior locations of MIP are more involved in spatial than limb encoding with stronger contralateral preference. Also it seems that these two regions have less similarities in saccade encoding than reach encoding.

In summary, we have shown that LFP oscillations in dPul and MIP encode not only spatial location of movement goals but also they are sensitive to the effector with which this movement was done (eye, right or left hand). Although the level of LFP limb-, eye- and spatial-tuning is dependent on the frequency band and behavioral state, but both MIP and dPul shows almost similar direction of tuning within each monkey. However, the similarity across monkeys is less than similarities across regions within each monkey.

Overall, since neuronal responses in both regions suggests contribution of both in visually-guided reaches, further investigations needed to reveal their causal role in shaping neuronal representations. The second part of this thesis is the first study to our knowledge that combined electrophysiology and pharmacological reversible inactivation to understand the causal role of pulvinar on the reachand saccade-related neuronal responses in cortical parietal areas. We pharmacologically inactivated pulvinar and simultaneously recorded from MIP (or LIP) in both hemispheres with similar anatomical locations, while monkey performed dissociated delay reach (or saccade) task.

Pulvinar inactivation caused mainly contralesional hand/space behavioral deficits with more effects on reaches than saccades and it decreased the performance. In general monkey had slower contralesional hand movements and unusual contralesional hand shapes and grasping posture, similar to a previous studies (Wilke et al., 2018, 2010). Pulvinar inactivation also caused ipsilesional choice bias only for reaches with contralateral arm but his saccade choice bias was just slightly biased (not significant) which could be due to low number of sessions. In addition, monkey was slower to initiate and also to move toward fixation spot (i.e., initiate movement from resting position) with contralesional hand and also his movement toward spatial targets was slower only with contralesional hand. Besides that, pulvinar inactivation only increased his saccadic reaction time toward contralesional targets more than ipsilesional side. This results show signature of spatial neglect and optic ataxia (Christopoulos et al., 2015b; Hwang et al., 2014; Kubanek \& Snyder, 2015b,b; Wardak et al., 2002; Wilke et al., 2012; Yttri et al., 2014) which have been seen as deficits after parietal lesions and also after pulvinar lesions (Wilke et al., 2018, 2010).

Apart from behavioral deficits, pulvinar inactivation severely changed neuronal responses (both LFP and spiking activity) in the parietal LIP and MIP areas. On the population level, while firing of most MIP units in the intact hemisphere increased after pulvinar inactivation, in the inactivated hemisphere both decrease and increase of firing was observed. This effect was consistent across different task epoch and across conditions (limb and space). This increase of firing in intact hemisphere might be because of disinhibitory effects from the inactivated hemisphere and for that cells in TRN in 
both hemispheres play a major role (Jones, 2009). These diverse changes might reflect functional impairment and compensatory mechanisms. However, in both intact and inactivated hemisphere, firing rate of LIP units did not change significantly at the population level which means similar number of cells showed decreased as well as increased firing rates. This implies that pulvinar might play a different role in mediating LIP activity for saccade than MIP activity for reaches which further suggests that pulvinar plays a stronger causal role in shaping reach signals. Further investigation is needed to reveal whether pulvinar inactivation had different effects on the hand and spatially tuned cells.

Pulvinar inactivation caused strong and consistent (across sites and sessions) changes also in the LFP power in MIP and LIP, both in the inactivated and the opposite hemisphere. Low frequency power (delta-theta-alpha) in the inactivated hemisphere significantly increased (mostly before movement) similar to previous observations (Neske, 2016; Zhou et al., 2016). It has been argued that in less attentive states or drowsiness (which we also observed after inactivation), reticular cells are active at peak and relay cells are firing bursts in a slow frequency rhythms which causes high amplitude slow waves of delta to alpha frequencies (2-15 Hz) (Jones, 2009). This implies that pulvinar might be important to maintain brain state in an alert and attentive mode.

Parietal alpha-beta band power was modulated after inactivation in a limb-specific manner although differently in MIP than LIP. In MIP, specifically during delay epoch, beta band power increased in the inactivated hemisphere for the contralesional hand and decreased for the ipsilesional hand. However in the intact hemisphere, low beta power decreased for the contralesional hand. But in LIP, beta band power decreased in all conditions regardless of hand. This effect might be because the monkey still uses his arms to fixate but not actively plan for a spatial reach target. In addition, we observed that pulvinar inactivation increased strongly in MIP high beta and gamma band activity in intact hemisphere in all conditions but in LIP stronger for the ipsilesional space. All together, these diverse changes might reflect both induced functional impairment and compensatory mechanisms that enable the monkey to successfully perform the tasks even after the inactivation although with performance.

In addition to modulations in the power of oscillatory activity in parietal cortex, based on our preliminary analysis, dysfunction of pulvinar disrupts coherent oscillations in theta and beta frequencies not only within inactivated hemisphere but also across two hemispheres. This suggests further that pulvinar plays a role in beta and theta band synchronization within parietal reach and saccade areas although it has been shown that pulvinar inactivation modulates gamma band synchronization in visual areas (Zhou et al., 2016). Interestingly the lower level of desynchronization in LIP than MIP might suggest that pulvinar mediate reach related areas stronger than saccade related areas although we cannot rule out the possibility that desynchronization in LIP might still be because of used-limb for fixation in saccade task.

Altogether our data shows that pulvinar plays an important role in visually-guided motor goal behavior and it is causally active in affecting parietal reach and saccade movement-planning areas by modulating firing rates, oscillatory activity and synchronization not only within ipsilateral hemisphere but also across two hemispheres. Our findings show that neuronal signals in dorsal pulvinar encode spatial as well as effector information during visual processing, motor planning and execution, showing partial similarities to parietal cortex. There are indications that pulvinar might be more strongly involved in mediating visually-guided reach signals than saccades, underscoring its putative 
role in coordinated visually-guided actions. Dorsal pulvinar is causally involved in parietal action selection and planning mechanisms, beyond the previously suggested notion that the pulvinar is important for maintaining "alert cortical state". 



\section{References}

Aboitiz, F. (1992). Brain connections: interhemispheric fiber systems and anatomical brain asymmetries in humans. Biological Research, 25(2):51-61.

Acuña, C., Cudeiro, J., and Gonzalez, F. (1986). Lateral-posterior (LP) and pulvinar unit activity related to intentional upper limb movements directed to spatially separated targets, in behaving Macaca nemestrina monkeys. Revue Neurologique, 142(4):354-361.

Acuna, C., Cudeiro, J., Gonzalez, F., Alonso, J. M., and Perez, R. (1990). Lateral-posterior and pulvinar reaching cells - comparison with parietal area 5a: a study in behaving Macaca nemestrina monkeys. Exp Brain Res, 82:158-166.

Acuna, C., Gonzalez, F., and Dominguez, R. (1983). Sensorimotor Unit Activity Related to Intention in the Pulvinar of Behaving Cebus Apella Monkeys. Exp Brain Res, 52:411-422.

Andersen, R. A. (1995). Encoding of intention and spatial location in the posterior parietal cortex. Cerebral Cortex, 5(5):457-469.

Andersen, R. A., Andersen, K. N., Hwang, E., and Hauschild, M. (2014). Optic ataxia: from Balint's syndrome to the parietal reach region. Neuron, 81(5):967-983.

Andersen, R. A. and Cui, H. (2009). Intention, Action Planning, and Decision Making in ParietalFrontal Circuits. Neuron, 63(5):568-583.

Archambault, P. S., Caminiti, R., and Battaglia-Mayer, A. (2009). Cortical Mechanisms for Online Control of Hand Movement Trajectory: The Role of the Posterior Parietal Cortex. Cerebral Cortex, 19(12):2848-2864.

Archambault, P. S., Ferrari-Toniolo, S., and Battaglia-Mayer, A. (2011). Online Control of Hand Trajectory and Evolution of Motor Intention in the Parietofrontal System. Journal of Neuroscience, 31(2):742-752.

Aron, A. R., Robbins, T. W., and Poldrack, R. A. (2014). Inhibition and the right inferior frontal cortex: one decade on. Trends in Cognitive Sciences, 18(4):177-185.

Aru, J., Aru, J., Priesemann, V., Wibral, M., Lana, L., Pipa, G., Singer, W., and Vicente, R. (2015). Untangling cross-frequency coupling in neuroscience. Current Opinion in Neurobiology, 31:51-61.

Bakola, S., Gamberini, M., Passarelli, L., Fattori, P., and Galletti, C. (2010). Cortical Connections of Parietal Field PEc in the Macaque: Linking Vision and Somatic Sensation for the Control of Limb Action. Cerebral Cortex, 20(11):2592-2604.

Band, G. P., Van Der Molen, M. W., and Logan, G. D. (2003). Horse-race model simulations of the stop-signal procedure. Acta psychologica, 112(2):105-142.

Barron, D. S., Eickhoff, S. B., Clos, M., and Fox, P. T. (2015). Human Pulvinar Functional Organization and Connectivity. Human brain mapping, 36(7):2417-2431. 
Battaglia-Mayer, A., Babicola, L., and Satta, E. (2016). Parieto-frontal gradients and domains underlying eye and hand operations in the action space. Neuroscience, 334:76-92.

Battaglia-Mayer, A., Ferrari-Toniolo, S., and Visco-Comandini, F. (2015). Timing and communication of parietal cortex for visuomotor control. Current Opinion in Neurobiology, 33:103-109.

Battaglia-Mayer, A., Ferrari-Toniolo, S., Visco-Comandini, F., Archambault, P. S., Saberi-Moghadam, S., and Caminiti, R. (2013). Impairment of online control of hand and eye movements in a monkey model of optic ataxia. Cerebral Cortex (New York, N.Y.: 1991), 23(11):2644-2656.

Battaglia-Mayer, A., Mascaro, M., Brunamonti, E., and Caminiti, R. (2005). The Over-representation of Contralateral Space in Parietal Cortex: A Positive Image of Directional Motor Components of Neglect? Cerebral Cortex, 15(5):514-525.

Battaglia-Mayer, A. and Caminiti, R. (2002). Optic ataxia as a result of the breakdown of the global tuning fields of parietal neurones. Brain, 125(2):225-237.

Becker, W. and Jürgens, R. (1979). An analysis of the saccadic system by means of double step stimuli. Vision Research, 19(9):967-983.

Belitski, A., Gretton, A., Magri, C., Murayama, Y., Montemurro, M. A., Logothetis, N. K., and Panzeri, S. (2008). Low-Frequency Local Field Potentials and Spikes in Primary Visual Cortex Convey Independent Visual Information. Journal of Neuroscience, 28(22):5696-5709.

Benarroch, E. E. (2015). Pulvinar: associative role in cortical function and clinical correlations. Neurology, 84(7):738-747.

Benjamini, Y. and Hochberg, Y. (1995). Controlling The False Discovery Rate: A Practical And Powerful Approach To Multiple Testing. Journal of the Royal Statistical Society. Series B (Methodological), 57(1):289-300.

Bertrand, O., Tallon-Baudry, C., Fischer, C., and Pernier, J. (2001). Object representation and gamma oscillations. page 8 .

Bisley, J. W. and Goldberg, M. E. (2010). Attention, Intention, and Priority in the Parietal Lobe. Annual Review of Neuroscience, 33(1):1-21.

Blatt, n., Wiseman, n., and Domany, n. (1996). Superparamagnetic clustering of data. Physical Review Letters, 76(18):3251-3254.

Bloom, J. S. and Hynd, G. W. (2005). The Role of the Corpus Callosum in Interhemispheric Transfer of Information: Excitation or Inhibition? Neuropsychology Review, 15(2):59-71.

Boecker, M., Drueke, B., Vorhold, V., Knops, A., Philippen, B., and Gauggel, S. (2011). When response inhibition is followed by response reengagement: An event-related fMRI study. Human Brain Mapping, 32(1):94-106.

Boecker, M., Gauggel, S., and Drueke, B. (2013). Stop or stop-change — Does it make any difference for the inhibition process? International Journal of Psychophysiology, 87(3):234-243.

Bokor, H., Frère, S. G. A., Eyre, M. D., Slézia, A., Ulbert, I., Lüthi, A., and Acsády, L. (2005). Selective GABAergic control of higher-order thalamic relays. Neuron, 45(6):929-940.

Boucher, L., Palmeri, T. J., Logan, G. D., and Schall, J. D. (2007). Inhibitory control in mind and brain: An interactive race model of countermanding saccades. Psychological Review, 114(2):376-397.

Brovelli, A., Ding, M., Ledberg, A., Chen, Y., Nakamura, R., and Bressler, S. L. (2004). Beta oscillations in a large-scale sensorimotor cortical network: Directional influences revealed by Granger causality. Proceedings of the National Academy of Sciences of the United States of America, 101(26):9849-9854. 
Buschman, T. J. and Miller, E. K. (2007). Top-down versus bottom-up control of attention in the prefrontal and posterior parietal cortices. Science (New York, N.Y.), 315(5820):1860-1862.

Buzsáki, G. (2002). Theta Oscillations in the Hippocampus. Neuron, 33(3):325-340.

Buzsáki, G., Anastassiou, C. A., and Koch, C. (2012). The origin of extracellular fields and currents - EEG, ECoG, LFP and spikes. Nature Reviews Neuroscience, 13(6):407-420.

Buzsáki, G. and Schomburg, E. W. (2015). What does gamma coherence tell us about inter-regional neural communication? Nature Neuroscience, 18(4):484-489.

Camalier, C., Gotler, A., Murthy, A., Thompson, K., Logan, G., Palmeri, T., and Schall, J. (2007). Dynamics of saccade target selection: Race model analysis of double step and search step saccade production in human and macaque. Vision Research, 47(16):2187-2211.

Caminiti, R., Chafee, M. V., Battaglia-Mayer, A., Averbeck, B. B., Crowe, D. A., and Georgopoulos, A. P. (2010). UNDERSTANDING THE PARIETAL LOBE SYNDROME FROM A NEUROPHYSIOLOGICAL AND EVOLUTIONARY PERSPECTIVE. The European journal of neuroscience, 31(12):2320-2340.

Caminiti, R., Genovesio, A., Marconi, B., Mayer, A. B., Onorati, P., Ferraina, S., Mitsuda, T., Giannetti, S., Squatrito, S., Maioli, M. G., and Molinari, M. (1999). Early coding of reaching: frontal and parietal association connections of parieto-occipital cortex. European Journal of Neuroscience, 11(9):3339-3345.

Caminiti, R., Innocenti, G. M., and Battaglia-Mayer, A. (2015). Organization and evolution of parietofrontal processing streams in macaque monkeys and humans. Neuroscience \& Biobehavioral Reviews, 56:73-96.

Caminiti, R. and Sbriccoli, A. (1985). The callosal system of the superior parietal lobule in the monkey. The Journal of Comparative Neurology, 237(1):85-99.

Canolty, R. T. and Knight, R. T. (2010). The functional role of cross-frequency coupling. Trends in Cognitive Sciences, 14(11):506-515.

Cappe, C., Morel, A., Barone, P., and Rouiller, E. M. (2009). The thalamocortical projection systems in primate: an anatomical support for multisensory and sensorimotor interplay. Cereb Cortex, 19(9):2025-37.

Cappe, C., Morel, A., and Rouiller, E. (2007). Thalamocortical and the dual pattern of corticothalamic projections of the posterior parietal cortex in macaque monkeys. Neuroscience, 146(3):1371-1387.

Chang, S. W. C., Calton, J. L., Lawrence, B. M., Dickinson, A. R., and Snyder, L. H. (2016). RegionSpecific Summation Patterns Inform the Role of Cortical Areas in Selecting Motor Plans. Cerebral Cortex, 26(5):2154-2166.

Chang, S. W. C., Dickinson, A. R., and Snyder, L. H. (2008). Limb-Specific Representation for Reaching in the Posterior Parietal Cortex. Journal of Neuroscience, 28(24):6128-6140.

Chang, S. W. C. and Snyder, L. H. (2012). The representations of reach endpoints in posterior parietal cortex depend on which hand does the reaching. Journal of Neurophysiology, 107(9):2352-2365.

Christopoulos, V., Bonaiuto, J., and Andersen, R. A. (2015a). A Biologically Plausible Computational Theory for Value Integration and Action Selection in Decisions with Competing Alternatives. PLOS Computational Biology, 11(3):e1004104.

Christopoulos, V. N., Bonaiuto, J., Kagan, I., and Andersen, R. A. (2015b). Inactivation of Parietal Reach Region Affects Reaching But Not Saccade Choices in Internally Guided Decisions. $J$ Neurosci, 35(33):11719-28. 
Christopoulos, V. N., Kagan, I., and Andersen, R. A. (2018). Lateral intraparietal area (LIP) is largely effector-specific in free-choice decisions. Scientific Reports, 8(1):8611.

Cisek, P. (2005). Neural representations of motor plans, desired trajectories, and controlled objects. Cognitive Processing, 6(1):15-24.

Cisek, P. (2007). Cortical mechanisms of action selection: the affordance competition hypothesis. Philosophical Transactions of the Royal Society of London. Series B, Biological Sciences, 362(1485):1585-1599.

Cisek, P. (2012). Making decisions through a distributed consensus. Current Opinion in Neurobiology, 22(6):927-936.

Cisek, P., Crammond, D. J., and Kalaska, J. F. (2003). Neural Activity in Primary Motor and Dorsal Premotor Cortex In Reaching Tasks With the Contralateral Versus Ipsilateral Arm. Journal of Neurophysiology, 89(2):922-942.

Cisek, P. and Kalaska, J. F. (2005). Neural Correlates of Reaching Decisions in Dorsal Premotor Cortex: Specification of Multiple Direction Choices and Final Selection of Action. Neuron, 45(5):801-814.

Cisek, P. and Kalaska, J. F. (2010). Neural Mechanisms for Interacting with a World Full of Action Choices. Annual Review of Neuroscience, 33(1):269-298.

Ciuparu, A. and Mureşan, R. C. (2016). Sources of bias in single-trial normalization procedures. European Journal of Neuroscience, 43(7):861-869.

Coe, B., Tomihara, K., Matsuzawa, M., and Hikosaka, O. (2002). Visual and anticipatory bias in three cortical eye fields of the monkey during an adaptive decision-making task. The Journal of Neuroscience: The Official Journal of the Society for Neuroscience, 22(12):5081-5090.

Colby, C. L. and Goldberg, M. E. (1999). Space and attention in parietal cortex. Annu Rev Neurosci, 22:319-49.

Crowell, A. L., Ryapolova-Webb, E. S., Ostrem, J. L., Galifianakis, N. B., Shimamoto, S., Lim, D. A., and Starr, P. A. (2012). Oscillations in sensorimotor cortex in movement disorders: an electrocorticography study. Brain: A Journal of Neurology, 135(Pt 2):615-630.

Cudeiro, J., González, F., Pérez, R., Alonso, J. M., and Acuña, C. (1989). Does the pulvinar-LP complex contribute to motor programming? Brain Research, 484(1-2):367-370.

Cui, H. and Andersen, R. A. (2007). Posterior Parietal Cortex Encodes Autonomously Selected Motor Plans. Neuron, 56(3):552-559.

Cui, H. and Andersen, R. A. (2011). Different Representations of Potential and Selected Motor Plans by Distinct Parietal Areas. Journal of Neuroscience, 31(49):18130-18136.

D. Logan, G. and B. Cowan, W. (1984). On the ability to inhibit thought and action: A theory of an act of control. Psychological Review, 91.

Danziger, S., Ward, R., Owen, V., and Rafal, R. (2002). The Effects of Unilateral Pulvinar Damage in Humans on Reflexive Orienting and Filtering of Irrelevant Information. Behavioural Neurology, 13(3-4):95-104.

de Lafuente, V., Jazayeri, M., and Shadlen, M. N. (2015). Representation of Accumulating Evidence for a Decision in Two Parietal Areas. Journal of Neuroscience, 35(10):4306-4318. 
Dea, M., Hamadjida, A., Elgbeili, G., Quessy, S., and Dancause, N. (2016). Different Patterns of Cortical Inputs to Subregions of the Primary Motor Cortex Hand Representation in Cebus apella. Cerebral Cortex (New York, NY), 26(4):1747-1761.

Dean, H. L., Hagan, M. A., and Pesaran, B. (2012). Only coherent spiking in posterior parietal cortex coordinates looking and reaching. Neuron, 73(4):829-841.

Destexhe, A. and Sejnowski, T. J. (2003). Interactions Between Membrane Conductances Underlying Thalamocortical Slow-Wave Oscillations. Physiological reviews, 83(4):1401-1453.

Ditterich, J. (2006). Evidence for time-variant decision making. European Journal of Neuroscience, 24(12):3628-3641.

Dominguez-Vargas, A.-U., Schneider, L., Wilke, M., and Kagan, I. (2017). Electrical Microstimulation of the Pulvinar Biases Saccade Choices and Reaction Times in a Time-Dependent Manner. The Journal of Neuroscience: The Official Journal of the Society for Neuroscience, 37(8):2234-2257.

Engel, A. K. and Fries, P. (2010). Beta-band oscillations-signalling the status quo? Current Opinion in Neurobiology, 20(2):156-165.

Engel, A. K., Fries, P., König, P., Brecht, M., and Singer, W. (1999). Temporal binding, binocular rivalry, and consciousness. Consciousness and Cognition, 8(2):128-151.

Engel, A. K., Konig, P., Kreiter, A. K., and Singer, W. (1991). Interhemispheric synchronization of oscillatory neuronal responses in cat visual cortex. Science, 252(5009):1177-1179.

Essen, D. V., Newsome, W. T., and Bixby, J. L. (1982). The pattern of interhemispheric connections and its relationship to extrastriate visual areas in the macaque monkey. Journal of Neuroscience, 2(3):265-283.

Fattori, P., Kutz, D. F., Breveglieri, R., Marzocchi, N., and Galletti, C. (2005). Spatial tuning of reaching activity in the medial parieto-occipital cortex (area V6a) of macaque monkey. The European Journal of Neuroscience, 22(4):956-972.

Filimon, F. (2010). Human cortical control of hand movements: parietofrontal networks for reaching, grasping, and pointing. The Neuroscientist: A Review Journal Bringing Neurobiology, Neurology and Psychiatry, 16(4):388-407.

Fitzpatrick, A. M., Dundon, N. M., and Valyear, K. F. (2018). The neural basis of hand choice: An fMRI investigation of the Posterior Parietal Interhemispheric Competition model. bioRxiv, page 409565 .

Fleming, S. M., Mars, R. B., Gladwin, T. E., and Haggard, P. (2009). When the Brain Changes Its Mind: Flexibility of Action Selection in Instructed and Free Choices. Cerebral Cortex, 19(10):2352-2360.

Fling, B. W., Bernard, J. A., Bo, J., and Langan, J. (2008). Corpus Callosum and Bimanual Coordination in Multiple Sclerosis. The Journal of neuroscience : the official journal of the Society for Neuroscience, 28(29):7248-7249.

Friedman-Hill, S. R., Robertson, L. C., Desimone, R., and Ungerleider, L. G. (2003). Posterior parietal cortex and the filtering of distractors. Proceedings of the National Academy of Sciences of the United States of America, 100(7):4263-4268.

Fries, P. (2005). A mechanism for cognitive dynamics: neuronal communication through neuronal coherence. Trends in Cognitive Sciences, 9(10):474-480.

Fries, P. (2015). Rhythms for Cognition: Communication through Coherence. Neuron, 88(1):220-235. 
Fries, P., Nikolić, D., and Singer, W. (2007). The gamma cycle. Trends in Neurosciences, 30(7):309316.

Fries, P., Reynolds, J. H., Rorie, A. E., and Desimone, R. (2001). Modulation of oscillatory neuronal synchronization by selective visual attention. Science, 291(5508):1560-1563.

Fry, A., Mullinger, K. J., O’Neill, G. C., Barratt, E. L., Morris, P. G., Bauer, M., Folland, J. P., and Brookes, M. J. (2016). Modulation of post-movement beta rebound by contraction force and rate of force development. Human Brain Mapping, 37(7):2493-2511.

Funahashi, S. (2017). Prefrontal Contribution to Decision-Making under Free-Choice Conditions. Frontiers in Neuroscience, 11.

Gail, A. and Andersen, R. A. (2006). Neural Dynamics in Monkey Parietal Reach Region Reflect Context-Specific Sensorimotor Transformations. Journal of Neuroscience, 26(37):9376-9384.

Gail, A., Klaes, C., and Westendorff, S. (2009). Implementation of spatial transformation rules for goal-directed reaching via gain modulation in monkey parietal and premotor cortex. The Journal of Neuroscience: The Official Journal of the Society for Neuroscience, 29(30):9490-9499.

Gamberini, M., Passarelli, L., Fattori, P., Zucchelli, M., Bakola, S., Luppino, G., and Galletti, C. (2009). Cortical connections of the visuomotor parietooccipital area V6ad of the macaque monkey. Journal of Comparative Neurology, 513(6):622-642.

Gaveau, V., Pélisson, D., Blangero, A., Urquizar, C., Prablanc, C., Vighetto, A., and Pisella, L. (2008). Saccade control and eye-hand coordination in optic ataxia. Neuropsychologia, 46(2):475-486.

Gazzaniga, M. S., Bogen, J. E., and Sperry, R. W. (1967). Dyspraxia Following Division of the Cerebral Commissures. Archives of Neurology, 16(6):606-612.

George, J. S., Strunk, J., Mak-McCully, R., Houser, M., Poizner, H., and Aron, A. R. (2013). Dopaminergic therapy in Parkinson's disease decreases cortical beta band coherence in the resting state and increases cortical beta band power during executive control. NeuroImage. Clinical, 3:261-270.

Gilbertson, T., Lalo, E., Doyle, L., Lazzaro, V. D., Cioni, B., and Brown, P. (2005). Existing Motor State Is Favored at the Expense of New Movement during 13-35 Hz Oscillatory Synchrony in the Human Corticospinal System. Journal of Neuroscience, 25(34):7771-7779.

Gold, J. I. and Shadlen, M. N. (2000). Representation of a perceptual decision in developing oculomotor commands. 404:5.

Gold, J. I. and Shadlen, M. N. (2007). The Neural Basis of Decision Making. Annual Review of Neuroscience, 30(1):535-574.

Goodale, M. A. and Milner, A. D. (1992). Separate visual pathways for perception and action. Trends in Neurosciences, 15(1):20-25.

Gorea, A., Rider, D., and Yang, Q. (2014). A Unified Comparison of Stimulus-Driven, Endogenous Mandatory and 'Free Choice' Saccades. PLoS ONE, 9(2):e88990.

Grabenhorst, F., Hernádi, I., and Schultz, W. (2012). Prediction of economic choice by primate amygdala neurons. Proceedings of the National Academy of Sciences, 109(46):18950-18955.

Greenhouse, I. and Wessel, J. R. (2013). EEG signatures associated with stopping are sensitive to preparation. Psychophysiology, 50(9):900-908.

Gremel, C. M. and Costa, R. M. (2013). Premotor cortex is critical for goal-directed actions. Frontiers in Computational Neuroscience, 7. 
Grieve, K. L., Acuna, C., and Cudeiro, J. (2000). The primate pulvinar nuclei:vision and action. Trends Neurosci, 23:35-39.

Guillery, R. W. and Sherman, S. M. (2002). The thalamus as a monitor of motor outputs. Philosophical Transactions of the Royal Society B: Biological Sciences, 357(1428):1809-1821.

Hadjidimitrakis, K., Bertozzi, F., Breveglieri, R., Bosco, A., Galletti, C., and Fattori, P. (2014). Common neural substrate for processing depth and direction signals for reaching in the monkey medial posterior parietal cortex. Cerebral Cortex (New York, N.Y.: 1991), 24(6):1645-1657.

Haegens, S., Nácher, V., Luna, R., Romo, R., and Jensen, O. (2011). alpha-Oscillations in the monkey sensorimotor network influence discrimination performance by rhythmical inhibition of neuronal spiking. Proceedings of the National Academy of Sciences, 108(48):19377-19382.

Hagan, M. A., Dean, H. L., and Pesaran, B. (2012). Spike-field activity in parietal area LIP during coordinated reach and saccade movements. Journal of Neurophysiology, 107(5):1275-1290.

Hanes, D. P. and Carpenter, R. (1999). Countermanding saccades in humans. Vision Research, 39(16):2777-2791.

Hardy, S. G. P. and Lynch, J. C. (1992). The Spatial Distribution of Pulvinar Neurons That Project to Two Subregions of the Inferior Parietal Lobule in the Macaque. Cereb Cortex, 2(3):217-230.

Hartwigsen, G., Bestmann, S., Ward, N. S., Woerbel, S., Mastroeni, C., Granert, O., and Siebner, H. R. (2012). Left Dorsal Premotor Cortex and Supramarginal Gyrus Complement Each Other during Rapid Action Reprogramming. Journal of Neuroscience, 32(46):16162-16171.

Hawellek, D. J., Wong, Y. T., and Pesaran, B. (2016). Temporal coding of reward-guided choice in the posterior parietal cortex. Proceedings of the National Academy of Sciences, 113(47):13492-13497.

Hensel, A., Wolf, H., Kruggel, F., Riedel-Heller, S. G., Nikolaus, C., Arendt, T., and Gertz, H. J. (2002). Morphometry of the corpus callosum in patients with questionable and mild dementia. Journal of Neurology, Neurosurgery, and Psychiatry, 73(1):59-61.

Hooks, B. M., Mao, T., Gutnisky, D. A., Yamawaki, N., Svoboda, K., and Shepherd, G. M. G. (2013). Organization of cortical and thalamic input to pyramidal neurons in mouse motor cortex. The Journal of neuroscience : the official journal of the Society for Neuroscience, 33(2):748-760.

Hwang, E., Hauschild, M., Wilke, M., and Andersen, R. (2012). Inactivation of the Parietal Reach Region Causes Optic Ataxia, Impairing Reaches but Not Saccades. Neuron, 76(5):1021-1029.

Hwang, E. J. and Andersen, R. A. (2009). Brain-control of movement execution onset using LFPs in posterior parietal cortex. The Journal of neuroscience : the official journal of the Society for Neuroscience, 29(45):14363-14370.

Hwang, E. J. and Andersen, R. A. (2011). Effects of visual stimulation on LFPs, spikes, and LFP-spike relations in PRR. Journal of Neurophysiology, 105(4):1850-1860.

Hwang, E. J. and Andersen, R. A. (2012). Spiking and LFP activity in PRR during symbolically instructed reaches. Journal of Neurophysiology, 107(3):836-849.

Hwang, E. J., Hauschild, M., Wilke, M., and Andersen, R. A. (2014). Spatial and temporal eye-hand coordination relies on the parietal reach region. J Neurosci, 34(38):12884-92.

Igarashi, J., Isomura, Y., Arai, K., Harukuni, R., and Fukai, T. (2013). A theta-gamma Oscillation Code for Neuronal Coordination during Motor Behavior. Journal of Neuroscience, 33(47):1851518530 . 
Jackson, S. R., Newport, R., Mort, D., and Husain, M. (2005). Where the eye looks, the hand follows; limb-dependent magnetic misreaching in optic ataxia. Current biology: $C B, 15(1)$ :42-46.

Jensen, O. and Colgin, L. L. (2007). Cross-frequency coupling between neuronal oscillations. Trends in Cognitive Sciences, 11(7):267-269.

Jensen, O., Goel, P., Kopell, N., Pohja, M., Hari, R., and Ermentrout, B. (2005). On the human sensorimotor-cortex beta rhythm: sources and modeling. NeuroImage, 26(2):347-355.

Jha, A., Nachev, P., Barnes, G., Husain, M., Brown, P., and Litvak, V. (2015). The Frontal Control of Stopping. Cerebral Cortex (New York, NY), 25(11):4392-4406.

Jirsa, V. and Müller, V. (2013). Cross-frequency coupling in real and virtual brain networks. Frontiers in Computational Neuroscience, 7.

Jones, E. G. (2009). Synchrony in the Interconnected Circuitry of the Thalamus and Cerebral Cortex. Annals of the New York Academy of Sciences, 1157(1):10-23.

Jurkiewicz, M. T., Gaetz, W. C., Bostan, A. C., and Cheyne, D. (2006). Post-movement beta rebound is generated in motor cortex: evidence from neuromagnetic recordings. NeuroImage, 32(3):1281-1289.

Kaas, J. H. and Lyon, D. C. (2007). Pulvinar contributions to the dorsal and ventral streams of visual processing in primates. Brain Res Rev, 55(2):285-96.

Kagan, I., Iyer, A., Lindner, A., and Andersen, R. A. (2010). Space representation for eye movements is more contralateral in monkeys than in humans. Proceedings of the National Academy of Sciences, 107(17):7933-7938.

Kajikawa, Y. and Schroeder, C. (2011). How Local Is the Local Field Potential? Neuron, 72(5):847858.

Karnath, H.-O., Himmelbach, M., and Küker, W. (2003). The cortical substrate of visual extinction. Neuroreport, 14(3):437-442.

Katzner, S., Nauhaus, I., Benucci, A., Bonin, V., Ringach, D. L., and Carandini, M. (2009). Local Origin of Field Potentials in Visual Cortex. Neuron, 61(1):35-41.

Kelly, P. A. and McCulloch, J. (1982). Effects of the putative GABAergic agonists, muscimol and THIP, upon local cerebral glucose utilisation. Journal of Neurochemistry, 39(3):613-624.

Kermadi, I., Liu, Y., and Rouiller, E. M. (2000). Do bimanual motor actions involve the dorsal premotor (PMd), cingulate (CMA) and posterior parietal (PPC) cortices? Comparison with primary and supplementary motor cortical areas. Somatosensory \& Motor Research, 17(3):255-271.

Ketz, N. A., Jensen, O., and O'Reilly, R. C. (2015). Thalamic pathways underlying prefrontal cortex-medial temporal lobe oscillatory interactions. Trends in Neurosciences, 38(1):3-12.

Khanna, P. and Carmena, J. M. (2015). Neural oscillations: beta band activity across motor networks. Current Opinion in Neurobiology, 32:60-67.

Kühn, A. A., Williams, D., Kupsch, A., Limousin, P., Hariz, M., Schneider, G.-H., Yarrow, K., and Brown, P. (2004). Event-related beta desynchronization in human subthalamic nucleus correlates with motor performance. Brain: A Journal of Neurology, 127(Pt 4):735-746.

Kinsbourne, M. (1977). Hemi-neglect and hemisphere rivalry. Advances in Neurology, 18:41-49.

Klaes, C., Westendorff, S., Chakrabarti, S., and Gail, A. (2011). Choosing Goals, Not Rules: Deciding among Rule-Based Action Plans. Neuron, 70(3):536-548. 
Klimesch, W. (2012). Alpha-band oscillations, attention, and controlled access to stored information. Trends in Cognitive Sciences, 16(12):606-617.

Kraskov, A., Dancause, N., Quallo, M. M., Shepherd, S., and Lemon, R. N. (2009). Corticospinal Neurons in Macaque Ventral Premotor Cortex with Mirror Properties: A Potential Mechanism for Action Suppression? Neuron, 64(6):922-930.

Kravitz, D. J., Saleem, K. S., Baker, C. I., Ungerleider, L. G., and Mishkin, M. (2013). The ventral visual pathway: An expanded neural framework for the processing of object quality. Trends in cognitive sciences, 17(1):26-49.

Kreiman, G., Hung, C. P., Kraskov, A., Quiroga, R. Q., Poggio, T., and DiCarlo, J. J. (2006). Object Selectivity of Local Field Potentials and Spikes in the Macaque Inferior Temporal Cortex. Neuron, 49(3):433-445.

Kuang, S., Morel, P., and Gail, A. (2015). Planning Movements in Visual and Physical Space in Monkey Posterior Parietal Cortex. Cerebral Cortex, page bhu312.

Kubanek, J., Li, J. M., and Snyder, L. H. (2015). Motor role of parietal cortex in a monkey model of hemispatial neglect. Proceedings of the National Academy of Sciences, 112(16):E2067-E2072.

Kubanek, J. and Snyder, L. H. (2015a). Reward-Based Decision Signals in Parietal Cortex Are Partially Embodied. Journal of Neuroscience, 35(12):4869-4881.

Kubanek, J. and Snyder, L. H. (2015b). Reward Size Informs Repeat-Switch Decisions and Strongly Modulates the Activity of Neurons in Parietal Cortex. Cerebral Cortex, page bhv230.

Kubanek, J., Wang, C., and Snyder, L. H. (2013). Neuronal responses to target onset in oculomotor and somatomotor parietal circuits differ markedly in a choice task. Journal of Neurophysiology, 110(10):2247-2256.

Lamantia, A. S. and Rakic, P. (1990). Cytological and quantitative characteristics of four cerebral commissures in the rhesus monkey. The Journal of Comparative Neurology, 291(4):520-537.

Lega, B., Burke, J., Jacobs, J., and Kahana, M. J. (2016). Slow-Theta-to-Gamma Phase-Amplitude Coupling in Human Hippocampus Supports the Formation of New Episodic Memories. Cerebral Cortex, 26(1):268-278.

Lehmann, S. J. and Scherberger, H. (2015). Spatial Representations in Local Field Potential Activity of Primate Anterior Intraparietal Cortex (AIP). PLOS ONE, 10(11):e0142679.

Lewis, J. W. and Van Essen, D. C. (2000). Corticocortical connections of visual, sensorimotor, and multimodal processing areas in the parietal lobe of the macaque monkey. J Comp Neurol, 428(1):112-137.

Li, L. and Ebner, F. F. (2006). Balancing bilateral sensory activity: callosal processing modulates sensory transmission through the contralateral thalamus by altering the response threshold. Experimental Brain Research, 172(3):397-415.

Little, S. and Brown, P. (2014). The functional role of beta oscillations in Parkinson's disease. Parkinsonism \& Related Disorders, 20 Suppl 1:S44-48.

Liu, J. and Newsome, W. T. (2006). Local Field Potential in Cortical Area MT: Stimulus Tuning and Behavioral Correlations. Journal of Neuroscience, 26(30):7779-7790.

Logan, G. D. and Burkell, J. (1986). Dependence and Independence in Responding to Double Stimulation: A Comparison of Stop, Change, and Dual-Task Paradigms. page 15.

Logothetis, N. K. (2008). What we can do and what we cannot do with fMRI. Nature, 453(7197):869. 
Logothetis, N. K., Pauls, J., Augath, M., Trinath, T., and Oeltermann, A. (2001). Neurophysiological investigation of the basis of the fMRI signal. Nature, 412(6843):150.

Lu, Y., Truccolo, W., Wagner, F. B., Vargas-Irwin, C. E., Ozden, I., Zimmermann, J. B., May, T., Agha, N. S., Wang, J., and Nurmikko, A. V. (2015). Optogenetically induced spatiotemporal gamma oscillations and neuronal spiking activity in primate motor cortex. Journal of Neurophysiology, 113(10):3574-3587.

Marconi, B. (2001). Eye-Hand Coordination during Reaching. I. Anatomical Relationships between Parietal and Frontal Cortex. Cerebral Cortex, 11(6):513-527.

Marcos, E. and Genovesio, A. (2016). Determining Monkey Free Choice Long before the Choice Is Made: The Principal Role of Prefrontal Neurons Involved in Both Decision and Motor Processes. Frontiers in Neural Circuits, 10.

Mars, R. B., Piekema, C., Coles, M. G. H., Hulstijn, W., and Toni, I. (2007). On the Programming and Reprogramming of Actions. Cerebral Cortex, 17(12):2972-2979.

McAlonan, K., Cavanaugh, J., and Wurtz, R. H. (2008). Guarding the gateway to cortex: attention in visual thalamus. Nature, 456(7220):391-394.

Medendorp, W. P. (2004). Integration of Target and Effector Information in Human Posterior Parietal Cortex for the Planning of Action. Journal of Neurophysiology, 93(2):954-962.

Meera, P., Wallner, M., and Otis, T. S. (2011). Molecular basis for the high THIP/gaboxadol sensitivity of extrasynaptic GABAA receptors. Journal of Neurophysiology, 106(4):2057-2064.

Michaels, J. A., Dann, B., Intveld, R. W., and Scherberger, H. (2015). Predicting Reaction Time from the Neural State Space of the Premotor and Parietal Grasping Network. The Journal of Neuroscience: The Official Journal of the Society for Neuroscience, 35(32):11415-11432.

Mochizuki, K. and Funahashi, S. (2014). Opposing history effect of preceding decision and action in the free choice of saccade direction. Journal of Neurophysiology, 112(4):923-932.

Mochizuki, K. and Funahashi, S. (2016). Prefrontal spatial working memory network predicts animal's decision making in a free choice saccade task. Journal of Neurophysiology, 115(1):127-142.

Mohajerani, M. H., Aminoltejari, K., and Murphy, T. H. (2011). Targeted mini-strokes produce changes in interhemispheric sensory signal processing that are indicative of disinhibition within minutes. Proceedings of the National Academy of Sciences, 108(22):E183-E191.

Mooshagian, E., Wang, C., Holmes, C. D., and Snyder, L. H. (2017). Single Units in the Posterior Parietal Cortex Encode Patterns of Bimanual Coordination. Cerebral Cortex (New York, N.Y.: 1991), pages 1-19.

Mundinano, I.-C., Fox, D. M., Kwan, W. C., Vidaurre, D., Teo, L., Homman-Ludiye, J., Goodale, M. A., Leopold, D. A., and Bourne, J. A. (2018). Transient visual pathway critical for normal development of primate grasping behavior. Proceedings of the National Academy of Sciences of the United States of America, 115(6):1364-1369.

Musallam, S. (2004). Cognitive Control Signals for Neural Prosthetics. Science, 305(5681):258-262.

Nachev, P., Rees, G., Parton, A., Kennard, C., and Husain, M. (2005). Volition and Conflict in Human Medial Frontal Cortex. Current Biology, 15(2):122-128.

Nachev, P., Wydell, H., O’Neill, K., Husain, M., and Kennard, C. (2007). The role of the presupplementary motor area in the control of action. NeuroImage, 36:T155-T163. 
Neske, G. T. (2016). The Slow Oscillation in Cortical and Thalamic Networks: Mechanisms and Functions. Frontiers in Neural Circuits, 9.

Neubert, F.-X., Mars, R. B., Buch, E. R., Olivier, E., and Rushworth, M. F. S. (2010). Cortical and subcortical interactions during action reprogramming and their related white matter pathways. Proceedings of the National Academy of Sciences, 107(30):13240-13245.

Nowicka, A. and Tacikowski, P. (2011). Transcallosal transfer of information and functional asymmetry of the human brain. Laterality, 16(1):35-74.

Obeso, I., Robles, N., Marrón, E. M., and Redolar-Ripoll, D. (2013). Dissociating the Role of the pre-SMA in Response Inhibition and Switching: A Combined Online and Offline TMS Approach. Frontiers in Human Neuroscience, 7.

Ohayon, S. and Tsao, D. Y. (2012). MR-guided stereotactic navigation. J Neurosci Methods, 204(2):389-97.

Oostenveld, R., Fries, P., Maris, E., and Schoffelen, J.-M. (2011). FieldTrip: Open Source Software for Advanced Analysis of MEG, EEG, and Invasive Electrophysiological Data.

Padberg, J., Recanzone, G., Engle, J., Cooke, D., Goldring, A., and Krubitzer, L. (2010). Lesions in Posterior Parietal Area 5 in Monkeys Result in Rapid Behavioral and Cortical Plasticity. Journal of Neuroscience, 30(39):12918-12935.

Padoa-Schioppa, C. (2011). Neurobiology of Economic Choice: A Good-Based Model. Annual Review of Neuroscience, 34(1):333-359.

Palmer, L. M., Schulz, J. M., Murphy, S. C., Ledergerber, D., Murayama, M., and Larkum, M. E. (2012). The Cellular Basis of GABAB-Mediated Interhemispheric Inhibition. Science, 335(6071):989-993.

Pandya, D. N., Karol, E. A., and Heilbronn, D. (1971). The topographical distribution of interhemispheric projections in the corpus callosum of the rhesus monkey. Brain Research, 32(1):31-43.

Pandya, D. N. and Vignolo, L. A. (1969). Interhemispheric projections of the parietal lobe in the rhesus monkey. Brain Research, 15(1):49-65.

Parnaudeau, S., O'Neill, P., Bolkan, S., Ward, R., Abbas, A., Roth, B., Balsam, P., Gordon, J., and Kellendonk, C. (2013). Inhibition of medio-dorsal thalamus disrupts thalamo-frontal connectivity and cognition. Neuron, 77(6):1151-1162.

Pastor-Bernier, A. and Cisek, P. (2011). Neural Correlates of Biased Competition in Premotor Cortex. Journal of Neuroscience, 31(19):7083-7088.

Paul, L. K., Brown, W. S., Adolphs, R., Tyszka, J. M., Richards, L. J., Mukherjee, P., and Sherr, E. H. (2007). Agenesis of the corpus callosum: genetic, developmental and functional aspects of connectivity. Nature Reviews. Neuroscience, 8(4):287-299.

Pesaran, B., Nelson, M. J., and Andersen, R. A. (2008). Free choice activates a decision circuit between frontal and parietal cortex. Nature, 453(7193):406-409.

Petersen, S. E., Robinson, D. L., and Keys, W. (1985). Pulvinar nuclei of the behaving rhesus monkey: visual responses and their modulation. Journal of Neurophysiology, 54(4):867-886.

Petersen, S. E., Robinson, D. L., and Morris, J. D. (1987). Contributions of the pulvinar to visual spatial attention. Neuropsychologia, 25(1A):97-105.

Pfurtscheller, G., Neuper, C., Brunner, C., and da Silva, F. L. (2005). Beta rebound after different types of motor imagery in man. Neuroscience Letters, 378(3):156-159. 
Pfurtscheller, G., Stancák, A., and Neuper, C. (1996). Post-movement beta synchronization. A correlate of an idling motor area? Electroencephalography and Clinical Neurophysiology, 98(4):281293.

Pinault, D. and Deschênes, M. (1992). Voltage-dependent 40-Hz oscillations in rat reticular thalamic neurons in vivo. Neuroscience, 51(2):245-258.

Platt, M. L. and Glimcher, P. W. (1999). Neural correlates of decision variables in parietal cortex. Nature, 400(6741):233-238.

Pouget, P. (2005). Chronometry of Visual Responses in Frontal Eye Field, Supplementary Eye Field, and Anterior Cingulate Cortex. Journal of Neurophysiology, 94(3):2086-2092.

Pouget, P., Logan, G. D., Palmeri, T. J., Boucher, L., Pare, M., and Schall, J. D. (2011). Neural Basis of Adaptive Response Time Adjustment during Saccade Countermanding. Journal of Neuroscience, 31(35):12604-12612.

Poulet, J. F. A., Fernandez, L. M. J., Crochet, S., and Petersen, C. C. H. (2012). Thalamic control of cortical states. Nature Neuroscience, 15(3):370.

Prevosto, V., Graf, W., and Ugolini, G. (2010). Cerebellar Inputs to Intraparietal Cortex Areas LIP and MIP: Functional Frameworks for Adaptive Control of Eye Movements, Reaching, and Arm/Eye/Head Movement Coordination. Cerebral Cortex, 20(1):214-228.

Purushothaman, G., Marion, R., Li, K., and Casagrande, V. A. (2012). Gating and control of primary visual cortex by pulvinar. Nature neuroscience, 15(6):905-912.

Quigley, M., Cordes, D., Turski, P., Moritz, C., Haughton, V., Seth, R., and Meyerand, M. E. (2003). Role of the Corpus Callosum in Functional Connectivity. American Journal of Neuroradiology, 24(2):208-212.

Quiroga, R. Q., Nadasdy, Z., and Ben-Shaul, Y. (2004). Unsupervised spike detection and sorting with wavelets and superparamagnetic clustering. Neural Computation, 16(8):1661-1687.

Rajalingham, R., Stacey, R. G., Tsoulfas, G., and Musallam, S. (2014). Modulation of neural activity by reward in medial intraparietal cortex is sensitive to temporal sequence of reward. Journal of Neurophysiology, 112(7):1775-1789.

Ramakrishnan, A., Byun, Y. W., Rand, K., Pedersen, C. E., Lebedev, M. A., and Nicolelis, M. A. L. (2017). Cortical neurons multiplex reward-related signals along with sensory and motor information. Proceedings of the National Academy of Sciences, 114(24):E4841-E4850.

Ramakrishnan, A., Chokhandre, S., and Murthy, A. (2010). Voluntary Control of Multisaccade Gaze Shifts During Movement Preparation and Execution. Journal of Neurophysiology, 103(5):24002416.

Ramakrishnan, A., Sureshbabu, R., and Murthy, A. (2012). Understanding How the Brain Changes Its Mind: Microstimulation in the Macaque Frontal Eye Field Reveals How Saccade Plans Are Changed. Journal of Neuroscience, 32(13):4457-4472.

Ramkumar, P., Dekleva, B., Cooler, S., Miller, L., and Kording, K. (2016). Premotor and Motor Cortices Encode Reward. PLOS ONE, 11(8):e0160851.

Rangel, A. and Clithero, J. A. (2012). Value normalization in decision making: theory and evidence. Current opinion in neurobiology, 22(6):970-981.

Ratcliff, R. and McKoon, G. (2008). The Diffusion Decision Model: Theory and Data for Two-Choice Decision Tasks. Neural Computation, 20(4):873-922. 
Ray, S. and Maunsell, J. H. (2010). Differences in gamma frequencies across visual cortex restrict their possible use in computation. Neuron, 67(5):885-896.

Ray, S. and Maunsell, J. H. R. (2011). Network Rhythms Influence the Relationship between Spike-Triggered Local Field Potential and Functional Connectivity. Journal of Neuroscience, 31(35):12674-12682.

Ray, S., Schall, J. D., and Murthy, A. (2004). Programming of double-step saccade sequences: Modulation by cognitive control. Vision Research, 44(23):2707-2718.

Rilling, J. K. and Insel, T. R. (1999). Differential expansion of neural projection systems in primate brain evolution. Neuroreport, 10(7):1453-1459.

Robinson, D. L., Petersen, S. E., and Keys, W. (1986). Saccade-related and visual activities in the pulvinar nuclei of the behaving rhesus monkey. Experimental Brain Research, 62(3):625-634.

Rossiter, H. E., Davis, E. M., Clark, E. V., Boudrias, M.-H., and Ward, N. S. (2014). Beta oscillations reflect changes in motor cortex inhibition in healthy ageing. Neuroimage, 91(100):360-365.

Rushworth, M., Hadland, K. A., Paus, T., and Sipila, P. K. (2002). Role of the Human Medial Frontal Cortex in Task Switching: A Combined fMRI and TMS Study. Journal of Neurophysiology, 87(5):2577-2592.

Saalmann, Y. B. and Kastner, S. (2011). Cognitive and perceptual functions of the visual thalamus. Neuron, 71(2):209-223.

Saalmann, Y. B., Pinsk, M. A., Wang, L., Li, X., and Kastner, S. (2012). The Pulvinar Regulates Information Transmission Between Cortical Areas Based on Attention Demands. Science, 337(6095):753-756.

Samuelsen, C. L., Gardner, M. P. H., and Fontanini, A. (2013). Thalamic contribution to cortical processing of taste and expectation. The Journal of neuroscience : the official journal of the Society for Neuroscience, 33(5):1815-1827.

Scherberger, H. and Andersen, R. A. (2007). Target Selection Signals for Arm Reaching in the Posterior Parietal Cortex. Journal of Neuroscience, 27(8):2001-2012.

Scherberger, H., Jarvis, M. R., and Andersen, R. A. (2005). Cortical Local Field Potential Encodes Movement Intentions in the Posterior Parietal Cortex. Neuron, 46(2):347-354.

Schmid, M. C., Singer, W., and Fries, P. (2012). Thalamic Coordination of Cortical Communication. Neuron, 75(4):551-552.

Schultz, W. (2014). Predictive Reward Signal of Dopamine Neurons. page 28.

Schultz, W. (2015). Neuronal Reward and Decision Signals: From Theories to Data. Physiological Reviews, 95(3):853-951.

Seltzer, B. and Pandya, D. N. (1983). The distribution of posterior parietal fibers in the corpus callosum of the rhesus monkey. Experimental Brain Research, 49(1):147-150.

Seltzer, B. and Pandya, D. N. (1986). Posterior parietal projections to the intraparietal sulcus of the rhesus monkey. Experimental Brain Research, 62(3):459-469.

Shadlen, M. N. and Newsome, W. T. (2001). Neural Basis of a Perceptual Decision in the Parietal Cortex (Area LIP) of the Rhesus Monkey. Journal of Neurophysiology, 86(4):1916-1936.

Shankar, S., Massoglia, D. P., Zhu, D., Costello, M. G., Stanford, T. R., and Salinas, E. (2011). Tracking the Temporal Evolution of a Perceptual Judgment Using a Compelled-Response Task. Journal of Neuroscience, 31(23):8406-8421. 
Shen, K., Mišić, B., Cipollini, B. N., Bezgin, G., Buschkuehl, M., Hutchison, R. M., Jaeggi, S. M., Kross, E., Peltier, S. J., Everling, S., Jonides, J., McIntosh, A. R., and Berman, M. G. (2015). Stable long-range interhemispheric coordination is supported by direct anatomical projections. Proceedings of the National Academy of Sciences of the United States of America, 112(20):64736478.

Sherman, S. M. (2017). Functioning of Circuits Connecting Thalamus and Cortex. Comprehensive Physiology, 7(2):713-739.

Sherman, S. M. and Guillery, R. W. (2002). The role of the thalamus in the flow of information to the cortex. Philosophical Transactions of the Royal Society B: Biological Sciences, 357(1428):16951708 .

Shipp, S. (2003). The functional logic of cortico-pulvinar connections. Philosophical Transactions of the Royal Society B: Biological Sciences, 358(1438):1605-1624.

Shipp, S., Blanton, M., and Zeki, S. (1998). A visuo-somatomotor pathway through superior parietal cortex in the macaque monkey: cortical connections of areas V6 and V6a. The European Journal of Neuroscience, 10(10):3171-3193.

Shumikhina, S. and Molotchnikoff, S. (1999). Pulvinar participates in synchronizing neural assemblies in the visual cortex, in cats. Neuroscience Letters, 272(2):135-139.

Singer, W. (1999). Neuronal Synchrony: A Versatile Code for the Definition of Relations? Neuron, 24(1):49-65.

Singer, W. and Gray, C. M. (1995). Visual Feature Integration and the Temporal Correlation Hypothesis. Annual Review of Neuroscience, 18(1):555-586.

Snow, J. C., Allen, H. A., Rafal, R. D., and Humphreys, G. W. (2009). Impaired attentional selection following lesions to human pulvinar: Evidence for homology between human and monkey. Proceedings of the National Academy of Sciences of the United States of America, 106(10):40544059.

Snyder, L. H., Batista, A. P., and Andersen, R. A. (1997). Coding of intention in the posterior parietal cortex. Nature, 386(6621):167-170.

Soares, J. G. M., Diogo, A. C. M., Fiorani, M., Souza, A. P. B., and Gattass, R. (2004). Effects of inactivation of the lateral pulvinar on response properties of second visual area cells in Cebus monkeys. Clinical and Experimental Pharmacology \& Physiology, 31(9):580-590.

Stanford, T. R., Shankar, S., Massoglia, D. P., Costello, M. G., and Salinas, E. (2010). Perceptual decision making in less than 30 milliseconds. Nature Neuroscience, 13(3):379-385.

Staudigl, T., Zaehle, T., Voges, J., Hanslmayr, S., Esslinger, C., Hinrichs, H., Schmitt, F. C., Heinze, H.-J., and Richardson-Klavehn, A. (2012). Memory signals from the thalamus: early thalamocortical phase synchronization entrains gamma oscillations during long-term memory retrieval. Neuropsychologia, 50(14):3519-3527.

Steriade, M., McCormick, D. A., and Sejnowski, T. J. (1993). Thalamocortical oscillations in the sleeping and aroused brain. Science, 262(5134):679-685.

Stetson, C. and Andersen, R. A. (2014). The Parietal Reach Region Selectively Anti-Synchronizes with Dorsal Premotor Cortex during Planning. The Journal of Neuroscience, 34(36):11948-11958.

Suárez, R., Gobius, I., and Richards, L. J. (2014). Evolution and development of interhemispheric connections in the vertebrate forebrain. Frontiers in Human Neuroscience, 8. 
Suriya-Arunroj, L. and Gail, A. (2015). I Plan Therefore I Choose: Free-Choice Bias Due to Prior Action-Probability but Not Action-Value. Frontiers in Behavioral Neuroscience, 9.

Swann, N. C., Cai, W., Conner, C. R., Pieters, T. A., Claffey, M. P., George, J. S., Aron, A. R., and Tandon, N. (2012). Roles for the pre-supplementary motor area and the right inferior frontal gyrus in stopping action: Electrophysiological responses and functional and structural connectivity. NeuroImage, 59(3):2860-2870.

Sweeney-Reed, C. M., Zaehle, T., Voges, J., Schmitt, F. C., Buentjen, L., Kopitzki, K., Esslinger, C., Hinrichs, H., Heinze, H.-J., Knight, R. T., and Richardson-Klavehn, A. (2014). Corticothalamic phase synchrony and cross-frequency coupling predict human memory formation. eLife, 3:e05352.

Szczepanski, S. M., Konen, C. S., and Kastner, S. (2010). Mechanisms of Spatial Attention Control in Frontal and Parietal Cortex. Journal of Neuroscience, 30(1):148-160.

Tanne-Gariepy, J., Rouiller, E. M., and Boussaoud, D. (2002). Parietal inputs to dorsal versus ventral premotor areas in the macaque monkey: evidence for largely segregated visuomotor pathways. Exp Brain Res, 145(1):91-103.

Uhlhaas, P. J. and Singer, W. (2006). Neural Synchrony in Brain Disorders: Relevance for Cognitive Dysfunctions and Pathophysiology. Neuron, 52(1):155-168.

Van der Stigchel, S., Arend, I., van Koningsbruggen, M. G., and Rafal, R. D. (2010). Oculomotor integration in patients with a pulvinar lesion. Neuropsychologia, 48(12):3497-504.

Varela, C. and Sherman, S. M. (2007). Differences in response to muscarinic activation between first and higher order thalamic relays. Journal of Neurophysiology, 98(6):3538-3547.

Verbruggen, F. and Logan, G. D. (2008a). After-effects of goal shifting and response inhibition: A comparison of the stop-change and dual-task paradigms. The Quarterly Journal of Experimental Psychology, 61(8):1151-1159.

Verbruggen, F. and Logan, G. D. (2008b). Response inhibition in the stop-signal paradigm. Trends in Cognitive Sciences, 12(11):418-424.

Verbruggen, F., Schneider, D. W., and Logan, G. D. (2008). How to stop and change a response: The role of goal activation in multitasking. Journal of Experimental Psychology: Human Perception and Performance, 34(5):1212-1228.

Waldert, S., Lemon, R. N., and Kraskov, A. (2013). Influence of spiking activity on cortical local field potentials: Influence of spikes on local field potential. The Journal of Physiology, 591(21):52915303.

Ward, R. and Danziger, S. (2005). Selective attention and response control following damage to the human pulvinar. In Humphreys, G. and Riddoch, J., editors, Attention in Action: Advances from Cognitive Neuroscience, pages 325-350. Psychology Press, New York.

Ward, R., Danziger, S., and Bamford, S. (2005). Response to visual threat following damage to the pulvinar. Curr Biol, 15(6):571-3.

Ward, R., Danziger, S., Owen, V., and Rafal, R. (2002). Deficits in spatial coding and feature binding following damage to spatiotopic maps in the human pulvinar. Nature Neuroscience, 5(2):99-100.

Wardak, C., Olivier, E., and Duhamel, J.-R. (2002). Saccadic Target Selection Deficits after Lateral Intraparietal Area Inactivation in Monkeys. J Neurosci, 22(22):9877-9884.

Waszczak, B. L., Hruska, R. E., and Walters, J. R. (1980). GABAergic actions of THIP in vivo and vitro: a comparison with muscimol and GABA. European Journal of Pharmacology, 65(1):21-29. 
Watson, B. O., Ding, M., and Buzsaki, G. (2017). Temporal coupling of field potentials and action potentials in the neocortex. bioRxiv, page 214650.

Wilke, M., Kagan, I., and Andersen, R. A. (2012). Functional imaging reveals rapid reorganization of cortical activity after parietal inactivation in monkeys. Proc Natl Acad Sci U S A, 109(21):8274-9.

Wilke, M., Kagan, I., and Andersen, R. A. (2013). Effects of pulvinar inactivation on spatial decision-making between equal and asymmetric reward options. J Cogn Neurosci, 25(8):1270-83.

Wilke, M., Schneider, L., Dominguez-Vargas, A.-U., Schmidt-Samoa, C., Miloserdov, K., Nazzal, A., Dechent, P., Cabral-Calderin, Y., Scherberger, H., Kagan, I., and Bähr, M. (2018). Reach and grasp deficits following damage to the dorsal pulvinar. Cortex; a Journal Devoted to the Study of the Nervous System and Behavior, 99:135-149.

Wilke, M., Turchi, J., Smith, K., Mishkin, M., and Leopold, D. A. (2010). Pulvinar Inactivation Disrupts Selection of Movement Plans. Journal of Neuroscience, 30(25):8650-8659.

Witelson, S. F. and Goldsmith, C. H. (1991). The relationship of hand preference to anatomy of the corpus callosum in men. Brain Research, 545(1-2):175-182.

Womelsdorf, T., Fries, P., Mitra, P. P., and Desimone, R. (2006). Gamma-band synchronization in visual cortex predicts speed of change detection. Nature, 439(7077):733-736.

Wong, Y. T., Fabiszak, M. M., Novikov, Y., Daw, N. D., and Pesaran, B. (2016). Coherent neuronal ensembles are rapidly recruited when making a look-reach decision. Nature Neuroscience, 19(2):327-334.

Wróbel, A., Ghazaryan, A., Bekisz, M., Bogdan, W., and Kamiński, J. (2007). Two Streams of Attention-Dependent beta Activity in the Striate Recipient Zone of Cat's Lateral Posterior-Pulvinar Complex. Journal of Neuroscience, 27(9):2230-2240.

Yamada, H., Louie, K., Tymula, A., and Glimcher, P. W. (2018). Free choice shapes normalized value signals in medial orbitofrontal cortex. Nature Communications, 9.

Yamauchi, H., Fukuyama, H., Ouchi, Y., Nagahama, Y., Kimura, J., Asato, R., and Konishi, J. (1995). Corpus callosum atrophy in amyotrophic lateral sclerosis. Journal of the Neurological Sciences, 134(1-2):189-196.

Yeterian, E. H. and Pandya, D. N. (1985). Corticothalamic connections of the posterior parietal cortex in the rhesus monkey. J Comp Neurol, 237(3):408-26.

Yeterian, E. H. and Pandya, D. N. (1989). Thalamic connections of the cortex of the superior temporal sulcus in the rhesus monkey. J Comp Neurol, 282(1):80-97.

Yeterian, E. H. and Pandya, D. N. (1997). Corticothalamic connections of extrastriate visual areas in rhesus monkeys. J Comp Neurol, 378(4):562-585.

Yttri, E. A., Wang, C., Liu, Y., and Snyder, L. H. (2014). The parietal reach region is limb specific and not involved in eye-hand coordination. Journal of Neurophysiology, 111(3):520-532.

Yu, C., Li, Y., Stitt, I. M., Zhou, Z. C., Sellers, K. K., and Frohlich, F. (2018). Theta Oscillations Organize Spiking Activity in Higher-Order Visual Thalamus during Sustained Attention. eNeuro, $5(1)$.

Zarei, M., Johansen-Berg, H., Smith, S., Ciccarelli, O., Thompson, A. J., and Matthews, P. M. (2006). Functional anatomy of interhemispheric cortical connections in the human brain. Journal of Anatomy, 209(3):311-320. 
Zhang, Y., Chen, Y., Bressler, S. L., and Ding, M. (2008). Response preparation and inhibition: The role of the cortical sensorimotor beta rhythm. Neuroscience, 156(1):238-246.

Zhou, H., Schafer, R., and Desimone, R. (2016). Pulvinar-Cortex Interactions in Vision and Attention. Neuron, 89(1):209-220. 



\section{Declaration}

I hereby declare that except where specific reference is made to the work of others, the contents of this dissertation are original and have not been submitted in whole or in part for consideration for any other degree or qualification in this, or any other university. This dissertation is my own work and contains nothing which is the outcome of work done in collaboration with others, except as specified in the text and Acknowledgments.

Danial Arabali

Göttingen, 2019

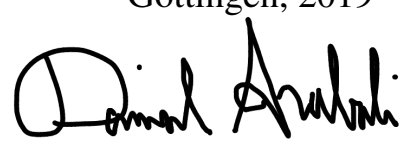





\section{Acknowledgements}

Here I would like to appreciate Dr. Igor Kagan and Prof. Melanie Wilke who gave me the opportunity to go through a unique experience in the field of cognitive neuroscience. I specifically thank Dr. Kagan, who is a real dedicated scientist, for his patient supports and for all of the experimental and analytical techniques he taught me. I would like also to be thankful to my thesis committee members Prof. Melanie Wilke and Prof. Hans Scherberger for their fruitful discussions and feedbacks in the last few years. I also thank Prof. Annekathrin Schacht, Dr. Arezoo Pooresmaeili and Prof. Alexander Gail that they kindly accepted to be in my examination board.

My special thanks go to previous and current lab colleagues and friends with whom I had many nice scientific and non-scientific moments. I would like to thank Malte Köster who gave me useful introduction to our psychophysics study; Dr. Caio Moreira who was not only my first friend in the lab but also a supportive, warmhearted and smart person with whom I experienced many things; Lydia Gibson with whom I had many conversations about social life and german culture; Lukas Schneider for his enormous technical supports, fruitful scientific discussions and feedbacks; Adan Ulises Domínguez-Vargas for his scientific feedbacks; Mathieu Pachoud for his scientific helps, nice personality and bringing a good spirit in the lab; and also especially thank Kristin Kaduk not only helped me scientifically but also for her warm personality, close friendship and many talks, discussions and good moments that we had together.

I also would like to express my gratitude to two our technical assistants: Ira Panolias who was the first to teach me animal handling and training, for her great technical supports and very nice character, and also Dr. Daniela Trinca Bertazzi Lazzarini who is very warmhearted and nice and was always available to help me in any respect. I would like also to thank other technical assistants of the Cognitive Neuroscience Lab for their unlimited helps: Beatrix Glaser, Leonore Burchardt, Sina Plümer, Ralf Brockhausen, Klaus Heisig, Matthis Drolet, Luisa Klotz, Janine Kuntze and Dirk Prüße. I would like to thank all my colleagues in the entire Cognitive Neuroscience Lab including Sensorimotor Group as well as Neurobiology Laboratory of prof. Scherberger who made the scientific and social atmosphere very friendly, pleasant and supportive.

Very especial thanks go to my macaque friends, Magnus, Tesla and Linus with whom I expended a big proportion of my time in the last few years and I learned many things from them.

In the end I would like to appreciate my family particularly my dear parents and also my friends for all of their emotional supports. And I would like to particularly thank my dear wife, Anna Kuhnt, who is a kindhearted and good friend and on top of that she has tolerated my tough times and supported me from the beginning. 